\title{
RISK MANAGEMENT
}

\section{MAGAZINE}

\author{
Anno 15, numero 3 \\ Settembre - Dicembre 2020
}

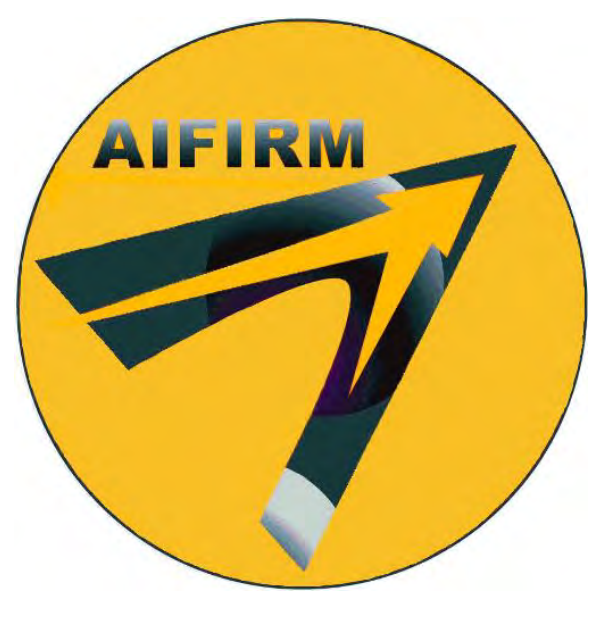

In collaborazione con

prometeia 


\section{IN QUESTO NUMERO}

\begin{tabular}{|l|l|}
\hline 4 & $\begin{array}{l}\text { Corporate Default Forecasting with Machine } \\
\text { Learning } \\
\text { Mirko Moscatelli, Simone Narizzano, Fabio } \\
\text { Parlapiano, Gianluca Viggiano }\end{array}$ \\
\hline 15 & $\begin{array}{l}\text { Modelli di business e modelli manageriali } \\
\text { della banca. Dal rischio di business model al } \\
\text { rischio strategico. Verso una revisione del } \\
\text { framework dei rischi bancari? } \\
\text { Maurizio Baravelli }\end{array}$ \\
\hline 29 & $\begin{array}{l}\text { Pandemic risk: operational aspects } \\
\text { Camilla Bello, Stefano Desando, Veruska Orio, } \\
\text { Paolo Giudici, Barbara Tarantino }\end{array}$ \\
\hline
\end{tabular}

ARTICLE SUBMITTED TO
DOUBLE-BLIND PEER REVIEW

\begin{tabular}{|l|l|}
\hline 33 & $\begin{array}{l}\text { The resilience of green stocks during COVID- } \\
\text { 19: a clustering approach } \\
\text { Giovanni Maria Bonagura, Luca D'Amico, Alessio } \\
\text { Iacopino, Lorenzo Prosperi, Lea Zicchino }\end{array}$ \\
\hline 48 & $\begin{array}{l}\text { Climate Change: EU taxonomy and forward } \\
\text { looking analysis in the context of emerging } \\
\text { climate related and environmental risks } \\
\text { Giuliana Birindelli, Vera Palea, Luca Trussoni, Fabio } \\
\text { Verachi }\end{array}$ \\
\hline 65 & $\begin{array}{l}\text { Critical analysis of the most widespread } \\
\text { methodologies for the simulation of the short } \\
\text { rate dynamics under extreme market } \\
\text { conditions } \\
\text { Pier Giuseppe Giribone }\end{array}$ \\
\hline 73 & $\begin{array}{l}\text { Blockchain securitization: an innovative } \\
\text { technology to boost asset liquidity } \\
\text { Valerio Begozzi, Francesco Dammacco, Paolo Fabris, } \\
\text { Gianmarco Fagiani, Chiara Frigerio, Riccardo } \\
\text { Rostagno, Angelo Santarossa }\end{array}$ \\
\hline
\end{tabular}

Rivista scientifica riconosciuta da ANVUR e AIDEA
Risk Management Magazine

Anno $15 n^{\circ} 3$ Settembre - Dicembre 2020

Direttore Responsabile (Chief Managing Editor)

Maurizio Vallino

Condirettore (Deputy Managing Editor)

Corrado Meglio

Editorial Board

Giampaolo Gabbi - Chief Editor Business Economics Area (SDA Bocconi); Paolo Giudici - Chief Editor Statistical Economics Area (Università di Pavia); Daniel Ahelegbey (Università di Pavia); Raffaella Calabrese (University of Edimburgh); Robert Eccles (Oxford University); Franco Fiordelisi (University of Essex); Pier Giuseppe Giribone (Università di Genova); Gulia Iori (London City University); Richard M. Levich (New York University); Michèle F. Sutter Rüdisser (University of San Gallen); Peter Schwendner (ZHAW Zurich University of Applied Sciences); Alessandra Tanda (Università di Pavia).

\section{Scientific Committee}

Arianna Agosto (Università di Pavia); Ruggero Bertelli (Università di Siena); Paola Bongini (Università Milano Bicocca); Anna Bottasso (Università di Genova); Marina Brogi (Università La Sapienza di Roma); Ottavio Caligaris (Università di Genova); Rosita Cocozza (Università di Napoli); Costanza Consolandi (Università di Siena); Simona Cosma (Università del Salento); Paola Ferretti (Università di Pisa); Andrea Giacomelli (Università di Venezia); Adele Grassi (Vice Presidente APB); Valentina Lagasio (Università La Sapienza di Roma); Duccio Martelli (Università di Perugia); Laura Nieri (Università di Genova); Pasqualina Porretta (Università La Sapienza di Roma); Anna Grazia Quaranta (Università di Macerata); Enzo Scannella (Università di Palermo); Cristiana Schena (Università dell'Insubria); Giuseppe Torluccio (Università di Bologna).

\section{Vignettista: Silvano Gaggero}

Proprietà, Redazione e Segreteria:

Associazione Italiana Financial Industry Risk Managers (AIFIRM), Via Sile 18, 20139 Milano

Registrazione del Tribunale di Milano n 629 del 10/9/2004

ISSN Print 2612-3665 - ISSN Online 2724-2153 DOI $10.47473 / 2016$ rrm

E-mail: risk.management.magazine@aifirm.it; Tel. +39 389 6946315

Stampa

Algraphy S.n.c. - Passo Ponte Carrega 62-62r 16141 Genova

Le opinioni espresse negli articoli impegnano unicamente la responsabilità dei rispettivi autori

SPEDIZIONE IN ABBONAMENTO POSTALE AI SOCI AIFIRM RESIDENTI IN ITALIA, IN REGOLA CON L'ISCRIZIONE

Rivista in stampa: 22 dicembre 2020 


\section{Peer review process on papers presented for publication}

The papers that are presented to our magazine for publication are submitted anonymously to a double level of peer review.

The first level is a review of eligibility, implemented on the paper by the members of the Scientific Council, who assess the adequacy of the paper to the typical topics of the magazine.

The second level is a review of suitability for publication, implemented on the paper by two referees, selected within the Scientific Council or externally among academics, scholars, experts on the subject who assess the content and the form.

\section{Editorial regulation}

"Risk Management Magazine" is the AIFIRM (Italian Association of Financial Industry Risk Managers) magazine, fully dedicated to risk management topics.

The organization includes the managing director, a joint manager and a Scientific Council composed by academics; the Council guarantees the quality and accuracy of the published articles.

The magazine promotes the diffusion of all content related to risk management topics, from regulatory aspects, to organizational and technical issues and all articles will be examined with interest through the Scientific Council.

The papers shall be presented in Microsoft Word format, font Times New Roman 10 and shall have between 20.000 and 100.000 characters; tables and graphs are welcome.

The bibliography shall be written in APA format and shall accuratel specify the sources.

An Abstract in Italian and one in English are required (less than 1200 characters) highlighting the Key words.

The authors bear sole responsibility for the opinions expressed in the articles.

The Statement on ethics and on unfair procedures in scientific publications can be found on our website www.aifirm.it.

\section{Processo di referaggio degli articoli proposti per la pubblicazione}

Gli articoli che sono proposti alla rivista per la pubblicazione sono sottoposti in forma anonima a due successivi livelli di referaggio. Il primo livello di referaggio (di ammissibilità) viene effettuato sull'articolo dai membri del Consiglio Scientifico che ne valutano la congruità ai temi trattati dalla rivista.

Il secondo livello di referaggio (di pubblicabilità) viene effettuato sull'articolo da due referee scelti all'interno del Consiglio Scientifico o all'esterno tra accademici, ricercatori, esperti della materia, che ne valutano il contenuto e la forma.

\section{Regolamento redazionale}

"Risk Management Magazine" è il periodico di AIFIRM (Associazione Italiana Financial Industry Risk Managers) ed interamente dedicato ai temi del risk management.

La sua struttura organizzativa prevede, oltre al direttore responsabile, un condirettore e un Consiglio Scientifico formato da accademici; quest'ultimo è garante della qualità e correttezza degli articoli pubblicati.

La rivista favorisce la diffusione di tutti i contenuti afferenti i temi del risk management, dagli aspetti normativi a quelli organizzativi e alle technicalities e vaglierà con interesse, per mezzo del Comitato Scientifico, i contributi che le perverranno.

Gli articoli proposti dovranno pervenire in formato Microsoft Word carattere Times New Roman 10 ed avere un numero di battute compreso tra 20.000 e 100.000; è gradita la presenza di tabelle e grafici.

La bibliografia deve essere redatta in formato APA, specificando accuratamente le fonti.

Si richiede la predisposizione di un Abstract in lingua italiana e in lingua inglese (meno di 1200 battute) con indicazione delle Key words.

Le opinioni espresse negli articoli impegnano unicamente la responsabilità dei rispettivi autori.

Sul sito www.aifirm.it è pubblicata la Dichiarazione sull'etica e sulle pratiche scorrette nella pubblicazione dei lavori scientifici. 


\section{Corporate Default Forecasting with Machine Learning}

Mirko Moscatelli, Simone Narizzano, Fabio Parlapiano and Gianluca Viggiano (Banca di Italia)

\section{Sommario}

Si presenta un raffronto tra modelli previsionali per il rischio di credito di tipo machine learning (ML) e modelli statistici standard quali la regressione logistica. Sfruttando un ampio dataset che include informazioni di bilancio e di Centrale dei Rischi per circa 300,000 imprese non finanziarie dal 2011 al 2017, si mostra come utilizzando soltanto informazioni di bilancio i modelli ML producono un incremento significativo in potere discriminate e precisione rispetto ai modelli statistici; il vantaggio si attenua quando si introducono anche le informazioni sul credito bancario.

\section{Abstract}

We compare statistical models usually employed in credit risk forecasting with machine learning algorithms (ML). Using a large dataset which includes financial ratios and credit behavioral indicators for about 300,000 Italian non-financial firms from 2011 to 2017, we show that training the models on financial statement data only, ML models record a significant improvement in discriminatory power and precision with respect to statistical models; however, this improvement is less pronounced when we enlarge the training dataset to include also credit behavioral data.

Keywords: credit scoring, machine learning, random forest, gradient boosting.

\section{Introduction}

Since the Basel II Accord, default forecasting methods based on a reduced-form regression approach have become popular in the banking industry. These methods consist of multivariate regression models which use firms' characteristics such as financial fundamentals to predict the credit quality of a firm. More recently, owing to the availability of large datasets and unstructured information, a growing strand of research suggests that models based on machine learning algorithms (henceforth ML) also constitute a suitable alternative for modelling default risk. ML refers to a class of models that can perform complex forecasting tasks when the relationship between predictors and the outcome is complex or unknown. As established in a number of works (Baesens et al., 2003; Brown and Mues, 2012; Barboza et al., 2017), ML models can perform highly accurate out-of-sample forecasts without imposing strong assumptions on the structure of the data generating process.

In this work we contrast statistical models with ensemble decision trees, a class of ML models which can handle both complex relationships across different variables and large datasets with correlated predictors. We use random forest (RDF) and gradient boosted tree (GBT) models, which combine a large number of predictions stemming from individual decision trees into a single (ensemble) forecast. The two models differ in the way individual trees are grown. In the RDF model, a random sample of the data and a random selection of variables are used for each tree in order to obtain less correlated individual predictions. The GBT model combines predictions obtained from trees that are tailored to deal sequentially with the forecasting errors of their predecessors.

We estimate the models using a large dataset covering financial and credit behavioral indicators for Italian non-financial firms. We test the out-of-sample performance for these competing models comparing one-year-ahead PD estimates and observed default data for the 2011-17 period.

Our analysis highlights the following main results:

(i) When using financial statement information usually available to external credit analysts, ML models outperform statistical models both in discriminatory power (the capacity to rank borrowers according to their riskiness) and precision (the ability to estimate PDs that deviate only marginally from actual default rates). When more information (namely from the Italian Credit Register) is added, gains from using ML are retained, albeit to a lesser extent.

(ii) We argue that improvements in forecasting performance from the use of ML are due to its capacity to exploit complex relationships between predictors and default outcomes.

\section{Related literature}

The use of the ML approach in credit risk modelling has gained momentum and has recently been applied in the field of early warning systems for banking crises, predictions of household mortgage or consumer credit default, and corporate default.

The most popular application of ML algorithms in default forecasting is in modelling consumer credit risk. Albanesi and Vamossy (2019) develop a model to predict consumer default based on deep learning (i.e. a combination of forecasts from deep neural network and gradient boosting) with high-dimensional data (over 200 variables). Deep learning models are shown to perform better than logistic regression and adapt to the behavior of the aggregate default rate quite closely. The model is able to capture the sharp rise in credit risk in the run-up to the 2007-09 crisis.

There are also numerous applications of ML to corporate default forecasting. Using a large dataset covering the North American corporate sector for the period 1987-2013, Barboza et al. (2017) show that ML provides an improvement of around 10 percentage points in AuROC over traditional models. In Bachman and Zhao (2017), ML models are compared to Moody's proprietary algorithm based on a regression model using corporate data for the United States; this exercise shows that ML models deliver an AuROC about 2-3 percentage points higher than a regression approach. However, their less transparent structure may lead to PDs that are difficult 
to relate to firms' underlying characteristics. Furthermore, the inclusion of credit behavioral variables in the predictor set notably increases the AuROC of each model by over 10 percentage points.

\section{Credit risk models at a glance}

We compare two types of default forecasting models: statistical and ML models. Statistical models are especially fit for the purpose of inference, and typically rely on assumptions regarding the structural relationships between variables, the number of parameters that can be robustly estimated, and the distribution properties of the data generating process. ML models are mostly focused on prediction accuracy and make very weak assumptions on the data generating process. This feature allows the detection of data-driven interactions and non-linear or non-monotonic relationships between predictors and outcome variable. This is particularly relevant to credit risk applications, but it comes at a cost of less transparency compared with statistical models: ${ }^{1}$ ML models do not provide estimates of the parameters that relate predictors to the outcome variable (the models are non-parametric) and this 'black box' feature can make their rationale and forecasts difficult to explain.

In this section, we briefly review standard credit risk models (3.1) and introduce the ML models used in the empirical application, namely random forest and gradient boosted trees (3.2).

\subsection{Statistical models}

Statistical theory offers a variety of methods for estimating default probabilities, of which linear discriminant analysis and logistic regression are the most popular. Linear discriminant analysis (LDA) provides an assessment of corporates' credit quality using a linear discriminant function that classifies borrowers into groups (default and non-default) based on their characteristics. For more than three decades discriminant analysis was used extensively by practitioners and performed reasonably well in predicting bankruptcy and other types of distress of privately and publicly held non-financial firms in the international context (Altman, 1968; Altman, 1983; Altman et al., 2017). However, criticism of its underlying assumptions (firms belonging to two different populations, normal distribution of observables, and equal covariance matrices for the two populations) gradually opened the field to more flexible models such as logistic regression.

The logistic regression model (LOG) estimates default probability from firms' observable characteristics modeling the default event as a Bernoulli random variable, assuming the value 0 for financially sound firms and 1 for defaulted firms. The model assumes that firms belong to the same population and that a known structural relationship (additive and linear) exists between the observable characteristics of the firm and the credit score. Model parameters are usually estimated via maximum likelihood. LOG relaxes some of discriminant analysis' assumptions (multivariate normality and equal covariance matrices), and has the significant advantage that its results can be easily interpreted.

These features attracted strong interest and prompted its diffusion as a scoring model amongst practitioners. ${ }^{2}$ Nevertheless, a number of limitations still apply: the lack of consideration of non-linear or complex interactions between observables and defaults, the sensitivity to outliers or missing data, and difficulties in fully exploiting large datasets. Penalized logistic regression model (PLR) has the same structural form as LOG, but it estimates parameters maximizing out-of-sample forecasting performance. Previous evidence shows that PLR can outperform standard logistic regression in some prediction tasks (Zou and Hastie, 2005).

Overall, statistical models are satisfactory forecasting devices that accommodate both accuracy and transparency requirements. This is owing to their plain functional form, which combines additively monotonic predictors of default into a probability with good out-of-sample performance.

However, the global financial crisis exposed some pitfalls in default forecasting based on commonly used rating systems approaches: i) their slow capacity to adapt to changes in the state of the economy, and ii) their limited ability to model complex nonlinear interactions between economic, financial and credit variables. For example, credit ratings may overlook signals of a deteriorating economic and credit environment, such as a rapid increase in default rates or negative shocks to the supply of credit.

\subsection{Machine learning models}

We applied two ML algorithms used extensively in credit risk applications: random forest (Breiman, 2001) and gradient boosted trees (Friedman, 2000). The building blocks for these models are classification trees, which are partition algorithms that recursively split the dataset into smaller sets (or branches) that best separate defaulters from non-defaulters. ${ }^{3}$ At each iteration, the decision tree algorithm chooses from the covariates space $\mathbf{X}$ a variable and a value for that variable, so as to minimize a measure of heterogeneity (impurity index) in the resulting subgroups with respect to the classification variable. ${ }^{4}$ The process continues within each branch until a stopping condition is reached, such as too few observations in the branch or no significant reduction in impurity. The final branches - called leaves - contain a constant estimate for the probability of default of firms in each leaf, computed as the proportion of defaulted firms over the total number of firms in that leaf in the estimation (or training) dataset.

\footnotetext{
${ }^{1}$ A recent line of research has put forward methods to increase the interpretability of ML models (see Guidotti et al., 2019). Moreover, the use of ML models for the purpose of inference is also considered in a number of works (see Chernozhukov et al., 2018 and Joseph, 2019).

${ }^{2}$ For example, the Bank of Italy's In-house Credit Assessment System (BI-ICAS), which is used to assess credit claims posted as collateral in Eurosystem monetary policy operations, is based on a standard logit framework. BI-ICAS integrates two credit risk measures: the credit behaviour component that models monthly data sourced from the Italian Credit Register; and the financial component, based on yearly financial statement data reported in the company accounts data archive of the Bank of Italy. The statistical model of the BI-ICAS estimates at monthly frequency around 300,000 default probabilities for Italian non-financial limited companies. The forecasting performance of the model is assessed annually by the Eurosystem.

${ }^{3}$ A good introduction to trees, random forest and boosting algorithms can be found in Hastie, Tibshirani and Friedman (2001).

${ }^{4} \mathrm{An}$ example of a measure of impurity, as well as the one that we use, is the Gini coefficient. Given a set of observations $\mathrm{S}$ with a binary value for each observation $y_{i} \in\{0,1\}$, the Gini impurity coefficient is defined as GI(S):=2· $\sum_{i} p_{i}\left(1-p_{i}\right)$, where $p_{i}$ is the percentage of observations in $S$ such that $y_{i}=1$. The Gini impurity coefficient ranges between 0 , when $p_{i}=0$ or $p_{i}=1$, and 0.5 when $p_{i}=0.5$.
} 
A simplified classification tree containing only two splitting variables $\mathrm{X}_{1}$ and $\mathrm{X}_{2}$ and four leaves is shown in Figure 1 . The first level splits the sample into two branches depending on the value of $X_{1}$. Firms with $X_{1}$ greater than 30 end up in a leaf and receive a default probability equal to 0.1 , whereas firms with $\mathrm{X}_{1}$ less than or equal to 30 are classified in a middle branch and are divided further according to the value of $X_{2}$. Firms with $X_{2}$ smaller than 50 end up in a leaf and receive a default probability equal to 0.6 , while firms with $\mathrm{X}_{2}$ greater or equal to 50 undergo a final split according to the value of $\mathrm{X}_{1}$ : if $\mathrm{X}_{1}$ is greater than 100 their default probability equals 0.4 ; otherwise, they receive a default probability of 0.2 .

Notice that the classification tree model: i) captures interactions between variables: the effect of $\mathrm{X}_{2}$ on the default probability strongly depends on the value of $\mathrm{X}_{1}$, for instance, if $\mathrm{X}_{1}$ is greater than 30 the value of $\mathrm{X}_{2}$ is irrelevant for the estimated default probability; ii) captures non-linear relationships, since the same variable can be used more than once with different values to split the tree, for example, $\mathrm{X}_{1}$ is used twice to split the sample; and iii) the default probabilities are not a continuous function of the variables: a small increase from 100 to 101 in the value of $\mathrm{X}_{1}$ results in the default probability of the firm going from 0.2 to 0.4 .

\section{Figure 1: Decision tree}

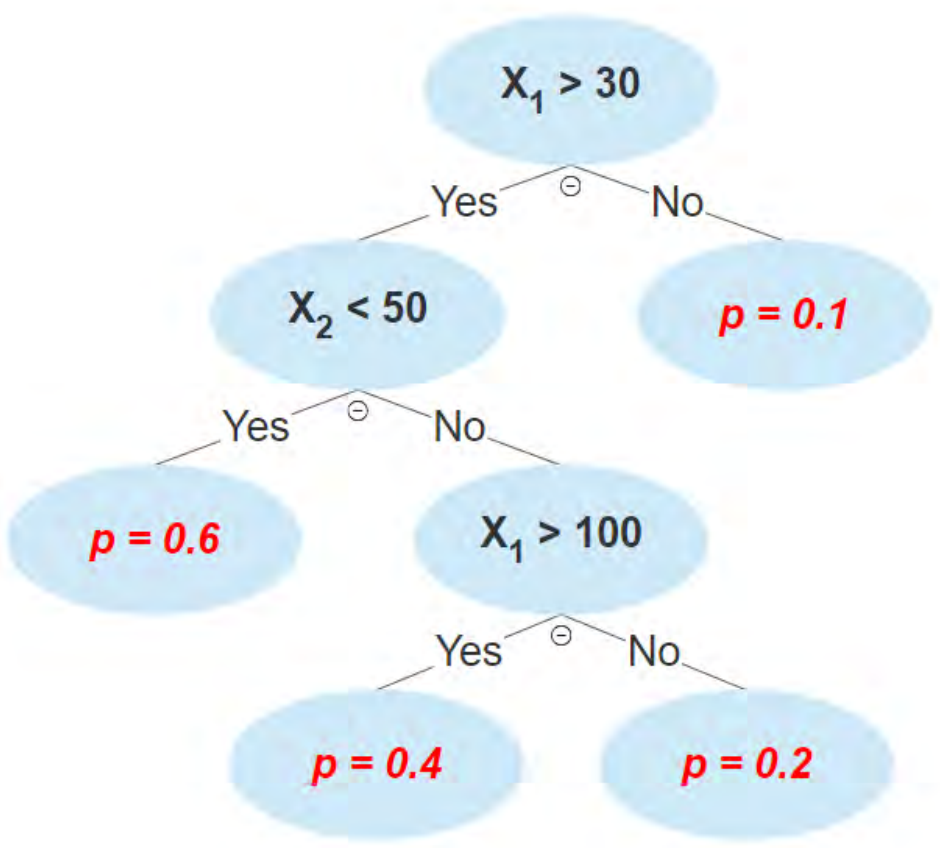

Notes: A decision tree for predicting firms' default probabilities grown using two variables, namely $X_{1}$ and $X_{2}$. Decision rules according to which branches are split are reported in black, while estimated default probabilities are reported in red.

Classification trees have the desirable property of being low-bias, meaning that the leaves, defined using multiple variables simultaneously, can fit the data extremely well. However, this flexibility results in an undesirable high-variance property, meaning that out-of-sample predictions are highly sensitive to small changes in the estimation dataset, which often leads to low forecasting power.

This shortcoming is addressed by ensemble classifiers, such as those provided by the random forest and gradient boosted tree models. Instead of using one single classification tree, these models grow a number of trees, and the final prediction is obtained as the average of the predictions stemming from the individual trees. In particular, the predictive function $\mathrm{F}$ takes the form of $\mathrm{F}(\mathrm{x})=\frac{1}{\mathrm{k}} \sum_{\mathrm{i}=1}^{\mathrm{k}} \mathrm{T}_{\mathrm{i}}(\mathrm{x})$, where $\left\{\mathrm{T}_{1}, \ldots, \mathrm{T}_{\mathrm{k}}\right\}$ are the $\mathrm{k}$ different classification trees and $\mathrm{T}_{\mathrm{i}}(\mathrm{x})$ is the probability of default that the tree $\mathrm{T}_{\mathrm{i}}$ associates to a borrower with covariates $\mathrm{x}$. The two models differ in how the different set of trees is constructed.

Random forest (RDF) grows the set of trees: i) using a different bootstrapped sample of the original dataset for each tree (i.e. a sample with replacement having the same number of observations of the original dataset); and ii) selecting at each branch the best split using only a randomly selected subset of the covariates. This procedure implies that the trees differ from one other, since the underlying information set is different.

Gradient boosted tree (GBT) grows the set of trees recursively: at each step classification errors from the previous trees are used as the dependent variable to grow the next tree. Namely, the first tree $T_{1}$ is a standard classification tree as described above; from the second tree onwards, trees are grown using the same $x$ covariate set, but a different dependent variable, computed as the difference between the $0 / 1$ default outcome and the estimations of the previous trees. ${ }^{5}$ In other words, the first tree $T_{1}$ will be trained on the model $y=T_{1}(x)$, the second tree $T_{2}$ will be trained on the model $y-T_{1}(x)=T_{2}(x)$, the third tree $T_{3}$ on the model $y-T_{1}(x)-$ $T_{2}(x)=T_{3}(x)$, and so on, each time trying to predict the forecasting errors of the previous trees. ${ }^{6}$ Iterated learning from previous

\footnotetext{
${ }^{5}$ Regression trees differ from classification trees because the output variables are continuous rather than numerical. They are generated in the same way, the only difference being that the impurity function is the variance of the outcome variable instead of the Gini impurity coefficient.

${ }^{6}$ Residuals can be interpreted as negative gradients in $\mathrm{F}$ of the quadratic loss function $\frac{1}{2}(\mathrm{y}-\mathrm{F}(\mathrm{x}))^{2}$ from which the name "gradient boosting" is derived.
} 
forecasting errors can achieve very accurate predictions, but can also lead to overfitting, thus the number of trees used is a very important hyperparameter to be chosen via cross-validation. ${ }^{7}$

\section{The training dataset}

\subsection{Corporate default}

We use an extensive dataset of financial and credit behavioral indicators for Italian non-financial firms for the period 2011-17. Our dependent variable, namely financial default, is sourced from the Italian Credit Register and reflects a system-wide definition of a borrower's non-performing status. A firm is classified in default on a given year if the ratio of non-performing credit to total credit drawn from the banking system is greater than 5 per cent for at least one month. ${ }^{8}$ The default rate, i.e. the ratio of borrowers classified as non-performing in a given year to the total number of borrowers not in default at the beginning of the year, gives an aggregate measure of credit risk which we aim to model at firm level (Table 1).

Within the 2011-17 time period, corporate sector credit risk peaked in 2014, in the aftermath of the European sovereign debt crisis and the associated slowdown of the Italian economy. Following the monetary policy measures adopted by the European Central Bank, the gradual improvement in the business cycle and the exit of vulnerable firms from the market, the aggregate default risk also decreased, with default rate levels approaching about 2.5 per cent in 2017.

The sudden increase in the number of defaults in both 2012 and 2014, which more than doubled compared to the previous year, may pose a challenge to slow-adapting credit risk models. From a qualitative point of view, the ability of models to forecast defaults in different stages of the credit cycle is a desirable property.

Table 1: Default rate

\begin{tabular}{ccc}
\hline \hline Year & N Firms & $\begin{array}{c}\text { Default } \\
\text { Rate }\end{array}$ \\
\hline 2011 & 222,879 & $1.20 \%$ \\
2012 & 233,157 & $2.45 \%$ \\
2013 & 259,166 & $2.23 \%$ \\
2014 & 249,566 & $4.76 \%$ \\
2015 & 252,059 & $4.12 \%$ \\
2016 & 260,156 & $3.31 \%$ \\
2017 & 269,657 & $2.53 \%$ \\
\hline \hline
\end{tabular}

Notes: Own calculation based on National Credit Register data. N Firms refers to the number of firms included in our sample, while Default rate is the proportion of firms in default in a given year over the number of firms not in default at the beginning of the year.

\subsection{Financial and credit behavioral indicators}

The majority of academic works on credit risk modelling use economic and financial ratios as potential indicators of corporate defaults. We add to these predictors a set of credit behavioral indicators on the firm-bank relationship.

Our dataset, drawn from the Company Accounts Data system (provided by Cerved Group) and the Italian Credit Register, contains a wide array of firm-level variables for Italian non-financial companies. Starting from financial ratios (time lag of one year), we compute 24 indicators covering: profitability, financing structure, debt sustainability and asset types. Credit behavioral indicators (with a time lag of two months) include eight variables related to a firm's financial flexibility, that is the proportion of drawn to granted bank credit for different facilities, and the occurrence of delinquencies within a firm-bank credit relationship. After including firms' descriptive indicators, such as economic sector and geographical area, our set of default predictors contains 38 variables. Variables were then selected using the following criteria:

1. using univariate logit regressions for the probability of default, variables with an AuROC lower than 55 per cent were dropped;

2. using the Kolmogorov-Smirnov test, variables with insignificant differences in the distributions between the default and non-default groups were dropped;

3. from the list satisfying 1) and 2), only less correlated variables were retained (linear correlation $<0.7$ ).

\footnotetext{
${ }^{7}$ Hyperparameters define the general characteristics of a model, such as its complexity, and can either be set a priori or learned from the data through optimizers such as grid search.

${ }^{8}$ The status of non-performing loans includes different stages of impairment: past-due 90 days, unlikely to pay and bad loans.
} 
We ended up with 26 predictors, and the estimation dataset includes about 250,000 yearly observations for defaulted and nondefaulted firms. A complete description of predictors can be found in the Appendix.

Variables displaying a linear relationship with the default rate are suitable candidates to be default predictors in all models. For example, financial leverage (equity over total assets) correlates linearly with the default rate (Figure 2, Panel A). ML models are expected to benefit more than statistical models from non-linear or non-monotonic indicators. For example, the sales growth rate displays a non-monotonic relationship with default risk, with low- and high- sales-growth firms being riskier (Figure 2, Panel B).

Figure 2: Default rate and predicting variables deciles
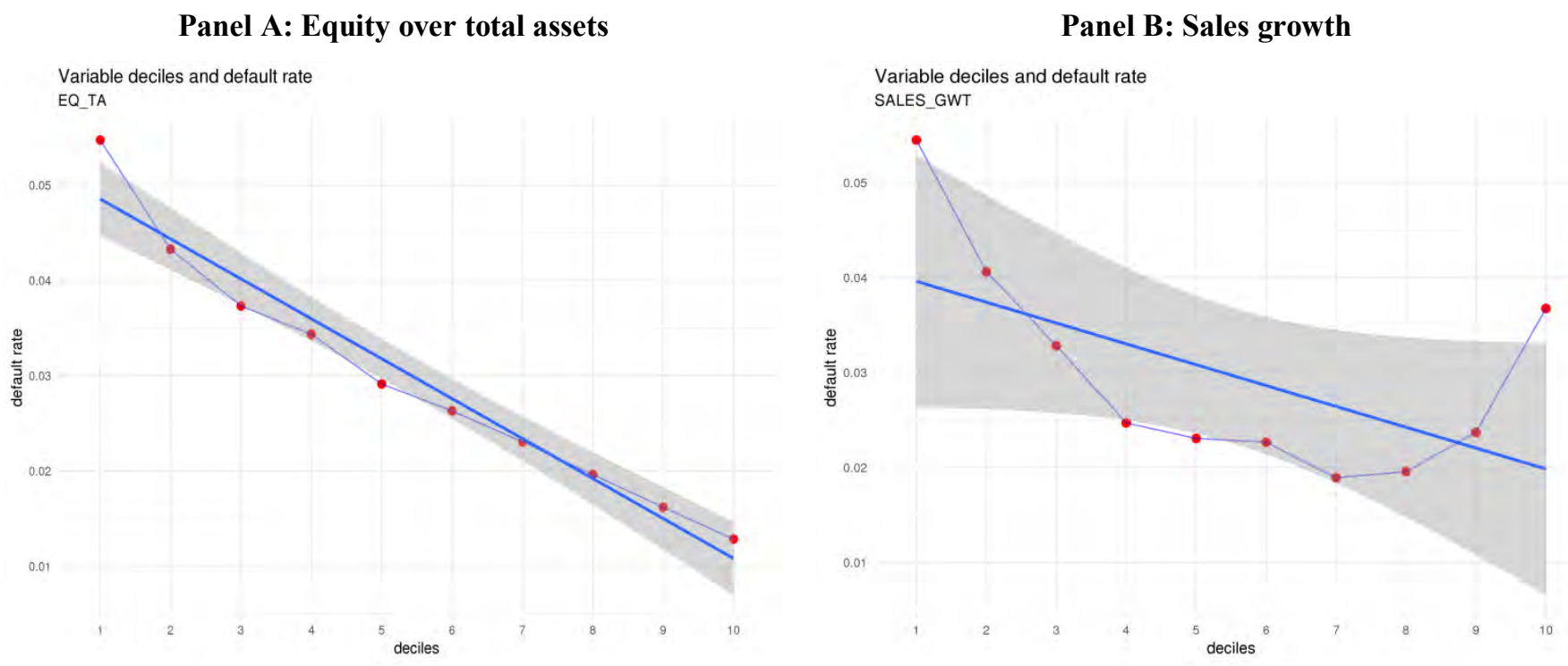

Notes: Own calculation based on National Credit Register and Cerved data.

Figure 2 plots (dots) the default rates associated with different deciles of two of the variables employed to predict defaults.

\section{Calibration}

The estimation of credit risk models is subject to the rare-event problem, i.e. the dataset usually only contains a small proportion of default observations compared to non-default observations, causing a weak discriminatory power: on the one hand, more importance is attributed to variables that identify sound firms (which are strongly represented in the estimation sample) rather than to variables that help distinguish distressed firms; on the other hand, the model tends to give very low PDs to all the firms.

Undersampling is commonly used to overcome this issue. In a first stage, the model is estimated on a balanced sample, namely a sample with an equal number of default and non-default observations, with the latter randomly sampled from the original dataset. ${ }^{9}$ Since this balanced sample does not reflect the real level of credit risk, during the second stage, a recalibration is performed via algebraic manipulation using Bayes correction (see Sugiyama et al., 2017; Dal Pozzolo et al., 2015). ${ }^{10}$

A second issue related to the estimation of ML models is hyperparameter calibration, which defines the general structure of the models. In RDF, we need to calibrate the number of variables selected at each split, whereas in GBT, we need to set the number of trees and leaves on each tree.

Using $k$-fold cross-validation, we choose the hyperparameters that maximize the out-of-sample performance. ${ }^{11}$ First, we randomly divide the training set into $k$ disjoint subsets (folds) of equal size; then, we train the model $k$ times, each time on a dataset composed by the union of different $k-1$ folds, and we use the remaining fold to compute an out-of-sample AuROC for the model. Finally, this gives us $k$ out-of-sample accuracy measures for each combination of the parameters, and we select the parameters presenting the highest average AuROC.

\section{Results}

In this section, we compare the credit risk models described in Section 2 based on discriminatory power and precision. First, we evaluate their performance using a limited training dataset, which includes financial indicators and firms' characteristics only. This dataset contains only publicly available financial information. Then we use the whole dataset, which covers both financial and credit behavioral information from the Italian Credit Register, with the latter set of indicators usually available only to lenders or supervisors.

\footnotetext{
${ }^{9}$ See Wallace and Dahabreh (2014).

${ }^{10}$ See Appendix 2 for a detailed description of the calibration procedure.

${ }^{11}$ For an introduction to cross-validation, see for example Geisser (1993).
} 


\subsection{Discriminatory power}

We assess the discriminatory power of the one-year default probabilities estimated by the different models with area under the curve (AuROC).$^{12}$ This is a measure of the ability of the model to assign higher default probabilities to firms that will default compared with financially sound firms: a random model that does not discriminate between sound and distressed firms has a 0.5 AuROC, while a perfect model has an AuROC of 1.

We first report the out-of-sample AuROC for the different models using only financial ratios and firm characteristics (such as geographic area, economic sector and firm size) that can be collected from publicly available sources (Table 2). The AuROC ranges from 72 to 77 per cent, a level of accuracy comparable to Wang and Dwyer (2011), Bacham and Zhao (2017), and Barboza et al., (2017).

We find that tree-based models outperform statistical models over the entire time span, with an average increase in discriminatory power over the LOG model of about 2.6 per cent. Linear discriminant analysis (LDA) and penalized logistic regression (PLR) display results very close to the LOG model.

Table 2: Discriminatory power with restricted dataset (financial indicators)

\begin{tabular}{cccccc}
\hline Year & $\begin{array}{c}\text { Linear } \\
\text { discriminant } \\
\text { analysis }\end{array}$ & $\begin{array}{c}\text { Logistic } \\
\text { regression }\end{array}$ & $\begin{array}{c}\text { Penalized } \\
\text { logistic } \\
\text { regression }\end{array}$ & Random forest & $\begin{array}{c}\text { Gradient } \\
\text { boosted trees }\end{array}$ \\
\hline 2012 & LDA & LOG & PLR & RDF & GBT \\
2013 & $73,7 \%$ & $73,9 \%$ & $73,9 \%$ & $76,6 \%$ & $76,3 \%$ \\
2014 & $73,7 \%$ & $73,9 \%$ & $73,9 \%$ & $77,2 \%$ & $77,3 \%$ \\
2015 & $73,7 \%$ & $73,7 \%$ & $73,7 \%$ & $76,1 \%$ & $76,0 \%$ \\
2016 & $72,6 \%$ & $72,6 \%$ & $72,6 \%$ & $75,3 \%$ & $75,3 \%$ \\
2017 & $73,0 \%$ & $73,0 \%$ & $73,0 \%$ & $75,7 \%$ & $75,4 \%$ \\
\hline
\end{tabular}

Notes: Own calculation based on Cerved data. The AuROC score is computed using out-of-sample Probabilities of defaults obtained from the various models and observed default data.

We then expand the training set to include also credit behavioral indicators. The AuROCs for these models are reported in Table 3 . We observe an increase in overall discriminatory power when credit behavioral indicators are included, amounting to about 10 percentage points in AuROC. This finding is consistent with Bacham and Zhao (2017), where credit behavioral indicators lead to similar increase in accuracy over a model based exclusively on financial ratios and firm characteristics. While still outperforming statistical models, treebased models now provide a smaller increase in discriminatory power over the logistic model. We interpret this finding as the effect of the different information set: with high quality data, the logistic regression already provides very good forecasting, approaching the upper bound in discriminatory power. As a result, the improvement in default forecasting owing to the use of ML is necessarily less pronounced.

${ }^{12}$ See Fawcett (2004), Chawla (2009) and Xu-Ying et al. (2009). 
Table 3: Discriminatory power with complete dataset (financial and credit behavioral indicators)

\begin{tabular}{cccccc}
\hline Year & $\begin{array}{c}\text { Linear } \\
\text { discriminant } \\
\text { analysis }\end{array}$ & $\begin{array}{c}\text { Logistic } \\
\text { regression }\end{array}$ & $\begin{array}{c}\text { Penalized } \\
\text { logistic } \\
\text { regression }\end{array}$ & Random forest & $\begin{array}{c}\text { Gradient } \\
\text { boosted trees }\end{array}$ \\
\hline 2012 & LDA & LOG & PLR & RDF & GBT \\
2013 & $83,8 \%$ & $84,0 \%$ & $84,0 \%$ & $84,6 \%$ & $84,7 \%$ \\
2014 & $83,2 \%$ & $83,3 \%$ & $83,3 \%$ & $84,2 \%$ & $84,4 \%$ \\
2015 & $81,1 \%$ & $81,6 \%$ & $81,6 \%$ & $82,5 \%$ & $82,7 \%$ \\
2016 & $82,8 \%$ & $82,9 \%$ & $82,9 \%$ & $84,4 \%$ & $84,6 \%$ \\
2017 & $82,9 \%$ & $83,0 \%$ & $83,0 \%$ & $84,1 \%$ & $84,0 \%$ \\
\hline
\end{tabular}

Notes: Own calculation based on Cerved and National Credit Register data. The AuROC score is computed using out-of-sample probabilities of defaults obtained from the various models and observed default data.

\subsection{Backtesting}

To assess the degree to which default probabilities match realized defaults, we perform a binomial-style test for different credit quality buckets, using the Credit Quality Steps (CQS) defined by the Eurosystem for validation and annual monitoring of credit rating systems. In particular, we use a backtesting strategy outlined in Coppens et al. (2017) as the 'traffic light approach': for each bucket, we test how often realized default rates are compatible with forecasted PDs and in the range of usual statistical deviations. A colour is assigned based on the p-value of the test, with green indicating that realized defaults are below the expected threshold, and yellow and red indicating that there is a positive or strongly positive discrepancy between expected and realized defaults respectively.

In Table 4 we report the results of the backtesting exercise for ML and statistical models trained on the full information set of financial and credit behavioral data: each cell reports the realized default rate within a certain PD bucket, and the PD thresholds represent the upper limit for PD in each interval.

For the years 2013 and 2015-17, characterized by declining default rates (Table 1), all rating systems report satisfactory performances. Structural models (LDA, LOG and PLR), however, show a lower capacity to correctly classify high credit quality borrowers, recording several red warnings in the CQSs 1-2 and 3. Tree-based models, instead, do not report significant discrepancies between expected and realized default for these years.

In the years 2012 and 2014, characterized by a strong increase in default rates, the estimated PDs often do not match realized defaults. Structural models tend to record weaker performances, presenting red warnings in all of the credit quality buckets.

Overall, these results show that the assessment of high credit quality borrowers and the adaptation to rapid deterioration in aggregate default risk is a common problem for structural models, while ML models can partially mitigate these issues. In particular, $\mathrm{RDF}$ predictions tend to be more precise compared to other models both in economic upturns and downturns.

\section{Conclusions}

We compared statistical models usually employed in credit risk modelling with ML models, namely random forest and gradient boosted tree models. We used a large dataset which includes financial ratios and credit behavioral indicators for about 300,000 Italian non-financial firms for the years 2011-17.

When the models are trained using only publicly available information, ML models have a more accurate forecasting performance, both in terms of discriminatory power and precision, compared with statistical models. This gain is reduced when high quality information, such as credit behavioral indicators, is added to the training set.

We argue that the better forecasting performance of ML models is due to their ability to capture more precisely the complex relationship between the available firms' indicators and the default outcome. Our results suggest that the joint use of statistical and ML models by lenders or credit analysts may be beneficial for the accurate assessment of potential borrowers. For example, ML models, which are relatively non-transparent, may be used as a benchmark for more transparent statistical models. 
Table 4: Backtesting

\begin{tabular}{cccccccccccc}
\hline \hline & & & 2012 & & & & & 2013 & \\
\hline CQS & Threshold & $L D A$ & $L O G$ & $P L R$ & $R D F$ & $G B M$ & $L D A$ & $L O G$ & $P L R$ & $R D F$ & $G B M$ \\
\hline CQS1-2 & $0,1 \%$ & $0,4 \%$ & $0,5 \%$ & $0,5 \%$ & $0,0 \%$ & $0,0 \%$ & $1,0 \%$ & $0,6 \%$ & $0,6 \%$ & $0,0 \%$ & $0,0 \%$ \\
CQS3 & $0,4 \%$ & $0,6 \%$ & $0,7 \%$ & $0,7 \%$ & $0,4 \%$ & $0,6 \%$ & $0,4 \%$ & $0,4 \%$ & $0,5 \%$ & $0,2 \%$ & $0,2 \%$ \\
CQS4 & $1 \%$ & $1,3 \%$ & $1,4 \%$ & $1,4 \%$ & $1,1 \%$ & $1,6 \%$ & $0,6 \%$ & $0,7 \%$ & $0,7 \%$ & $0,5 \%$ & $0,6 \%$ \\
CQS5 & $1,5 \%$ & $2,3 \%$ & $2,5 \%$ & $2,5 \%$ & $2,3 \%$ & $3,1 \%$ & $0,9 \%$ & $1,1 \%$ & $1,0 \%$ & $0,8 \%$ & $1,2 \%$ \\
CQS6 & $3 \%$ & $4,4 \%$ & $4,5 \%$ & $4,5 \%$ & $4,5 \%$ & $4,9 \%$ & $1,7 \%$ & $1,9 \%$ & $1,8 \%$ & $1,5 \%$ & $2,0 \%$ \\
CQS7 & $5 \%$ & $9,0 \%$ & $9,0 \%$ & $9,0 \%$ & $8,9 \%$ & $8,1 \%$ & $3,4 \%$ & $3,5 \%$ & $3,5 \%$ & $3,1 \%$ & $3,6 \%$ \\
\hline & & & & 2014 & & & & & 2015 & & \\
\hline CQS & Threshold & $L D A$ & $L O G$ & $P L R$ & $R D F$ & $G B M$ & $L D A$ & $L O G$ & $P L R$ & $R D F$ & $G B M$ \\
\hline CQS1-2 & $0,1 \%$ & $0,5 \%$ & $0,7 \%$ & $0,7 \%$ & $0,0 \%$ & $0,0 \%$ & $4,8 \%$ & $2,7 \%$ & $2,0 \%$ & $0,5 \%$ & $\mathrm{NA}$ \\
CQS3 & $0,4 \%$ & $1,0 \%$ & $1,1 \%$ & $1,0 \%$ & $0,2 \%$ & $0,5 \%$ & $0,9 \%$ & $0,8 \%$ & $0,8 \%$ & $0,1 \%$ & $0,1 \%$ \\
CQS4 & $1 \%$ & $1,4 \%$ & $1,6 \%$ & $1,6 \%$ & $0,7 \%$ & $1,4 \%$ & $0,9 \%$ & $0,9 \%$ & $0,9 \%$ & $0,3 \%$ & $0,5 \%$ \\
CQS5 & $1,5 \%$ & $2,1 \%$ & $2,3 \%$ & $2,3 \%$ & $1,5 \%$ & $2,7 \%$ & $0,9 \%$ & $1,1 \%$ & $1,1 \%$ & $0,6 \%$ & $1,0 \%$ \\
CQS6 & $3 \%$ & $3,3 \%$ & $3,4 \%$ & $3,3 \%$ & $3,2 \%$ & $4,1 \%$ & $1,7 \%$ & $1,8 \%$ & $1,8 \%$ & $1,4 \%$ & $1,9 \%$ \\
CQS7 & $5 \%$ & $5,4 \%$ & $5,6 \%$ & $5,6 \%$ & $6,3 \%$ & $6,4 \%$ & $2,8 \%$ & $2,8 \%$ & $2,8 \%$ & $3,1 \%$ & $3,5 \%$ \\
\hline & & & & 2016 & & & & & 2017 & & \\
\hline CQS & Threshold & $L D A$ & $L O G$ & $P L R$ & $R D F$ & $G B M$ & $L D A$ & $L O G$ & $P L R$ & $R D F$ & $G B M$ \\
\hline CQS1-2 & $0,1 \%$ & $0,0 \%$ & $6,4 \%$ & $7,9 \%$ & $0,0 \%$ & NA & $12,5 \%$ & $2,0 \%$ & $1,8 \%$ & $0,4 \%$ & $0,0 \%$ \\
CQS3 & $0,4 \%$ & $1,1 \%$ & $1,0 \%$ & $1,0 \%$ & $0,0 \%$ & $0,1 \%$ & $0,5 \%$ & $0,5 \%$ & $0,4 \%$ & $0,1 \%$ & $0,2 \%$ \\
CQS4 & $1 \%$ & $0,8 \%$ & $0,8 \%$ & $0,8 \%$ & $0,3 \%$ & $0,6 \%$ & $0,7 \%$ & $0,7 \%$ & $0,7 \%$ & $0,3 \%$ & $0,6 \%$ \\
CQS5 & $1,5 \%$ & $1,0 \%$ & $1,1 \%$ & $1,1 \%$ & $0,8 \%$ & $1,1 \%$ & $1,0 \%$ & $1,0 \%$ & $1,1 \%$ & $0,7 \%$ & $1,2 \%$ \\
CQS6 & $3 \%$ & $1,8 \%$ & $1,9 \%$ & $1,8 \%$ & $1,6 \%$ & $2,2 \%$ & $1,7 \%$ & $1,7 \%$ & $1,6 \%$ & $1,7 \%$ & $2,2 \%$ \\
CQS7 & $5 \%$ & $3,6 \%$ & $3,5 \%$ & $3,5 \%$ & $3,5 \%$ & $3,9 \%$ & $3,7 \%$ & $3,7 \%$ & $3,7 \%$ & $3,6 \%$ & $3,7 \%$ \\
\hline \hline
\end{tabular}

Notes: Own calculation. The Threshold column reports the upper limit of the CQS interval identified by the Euro Credit Assessment Framework (ECAF) scale. For instance, a firm is classified in CQS3 if the default probability is between 0.1 and 0.4 per cent. The percentages in the colored cells represent the realized default rate for each CQS in each year. The green, yellow and red colors denote the p-value of the "traffic light approach" test; where the H0 hypothesis is that true probabilities of defaults are less than or equal to the thresholds. The green shading indicates a p-value greater than 20 per cent, the yellow shading between 1 and 20 per cent, and the red shading less than 1 per cent.

Mirko Moscatelli, Simone Narizzano, Fabio Parlapiano and Gianluca Viggiano 


\section{References}

- Albanesi, S. \& D.F. Vamossy (2019). Predicting Consumer Default: A Deep Learning Approach. NBER Working Papers, No. 26165.

- Altman, E.I. (1968, September). Financial Ratios, Discriminant Analysis and the Prediction of Corporate Bankruptcy. Journal of Finance, 23, 589-609.

- Altman, E.I. (1983). Corporate Financial Distress: A Complete Guide to Predicting, Avoiding and Dealing With Bankruptcy. New York: John Wiley \& Sons.

- Altman, E.I., M. Iwanicz-Drozdowska, E.K. Laitinen \& A. Suvas (2017, June). Financial Distress Prediction in an International Context: A Review and Empirical Analysis of Altman's Z-Score Model. Journal of International Financial Management and Accounting, $28,31-171$.

- Bacham D. \& J. Zhao (2017, July). Machine Learning: Challenges, Lessons, and Opportunities in Credit Risk Modeling. Moody's Analytics Risk Perspectives, 9, 30-35.

- Baesens, B., T. Van Gestel, S. Viaene, M. Stepanova, J. Suykens \& J. Vanthienen (2003, June). Benchmarking state-of-the-art classification algorithms for credit scoring. Journal of the Operational Research Society, 54, 627-635.

- Barboza, F., H. Kimura \& E. Altman (2017, October). Machine learning models and bankruptcy prediction. Expert Systems with Applications: An International Journal, $\quad$ 83, 405-417.

- Breiman, L. (2001, October). Random Forests. Machine Learning, 45, 5-32.

- Brown, I. \& C. Mues (2012, February). An experimental comparison of classification algorithms for imbalanced credit scoring data sets. Expert Systems with Applications, 39, 3446-3453.

- Chawla, N.V. (2009). Data Mining for Imbalanced Datasets: An Overview. In O. Maimon and L. Rokach (Eds.), Data Mining and Knowledge Discovery Handbook (pp. 875-886). Boston: Springer.

- Chernozhukov, V., D. Chetverikov, M. Demirer, E. Duflo, C. Hansen, W. Newey \& J. Robins (2018, February). Double/debiased machine learning for treatment and structural parameters. The Econometrics Journal, 21, C1-C68.

- Coppens, F., F. González \& G. Winkler (2017). The performance of credit rating systems in the assessment of collateral used in Eurosystem monetary policy operations. European Central Bank Occasional Paper Series, No. 65.

- Dal Pozzolo, A., O. Caelen \& G. Bontempi (2015). When is Undersampling Effective in Unbalanced Classification Tasks?. In Joint European Conference on Machine Learning and Knowledge Discovery in Databases (pp. 200-215). Cham: Springer.

- Fawcett, T. (2004, September). ROC graphs: Notes and practical considerations for researchers. Machine Learning, $31,1-38$.

- Friedman, J.H. (2000, October). Greedy function approximation: A gradient boosting machine. The Annals of Statistics, 29, $1189-1232$.

- Friedman, J., T. Hastie \& R. Tibshirani (2001). The Elements of Statistical Learning: Data Mining, Interference, and Prediction. New York: Springer.

- Geisser, S. (1993). Predictive Inference: An Introduction. Monographs on Statistics and Applied Probability, 55.

- Guidotti, R., A. Monreale, S. Ruggieri, F. Turini, D. Pedreschi \& F. Giannotti (2019, January). A Survey of Methods for Explaining Black Box Models. ACM computing surveys, 51, 93.

- Joseph, A. (2019). Shapley regressions: A framework for statistical inference on machine learning models, Bank of England Working Papers, No. 784.

- Sugiyama, Masashi, Neil D. Lawrence \& Anton Schwaighofer (2018). Dataset shift in machine learning. Cambridge: The MIT Press.

- Wallace, B.C., and I.J. Dahabreh (2014, October). Improving class probability estimates for imbalanced data. Knowledge and Information Systems, 41, 33-52.

- Wang, J. \& D.W. Dwyer (2011). Moody's Analytics RiskCalc ${ }^{\mathrm{TM}}$ v3.1 Italy, Moody's Analytics.

- Xu-Ying, L., Wu, J. \& Zhou, Z.H. (2009, October). Exploratory Undersampling for Class-Imbalance Learning. IEEE Transactions on Systems, Man, and Cybernetics, Part B (Cybernetics), 39, 539-550. 


\section{Appendix}

We describe the financial and credit behavioral indicators used to predict default of non-financial firms. By means of graphical inspection, those variables presenting a non-linear or non-monotonous relationship with respect to the default outcome are labelled "NL".

\begin{tabular}{l} 
Variable \\
\hline TURNOVER (Asset Turnover Ratio) \\
VA_TA (Value Added to Total Assets) \\
EBITDA_MARGIN (EBITDA to Net Sales) - \\
$\boldsymbol{N} \boldsymbol{L}$ \\
PFN/PN (Net Debt to Equity) \\
EQ_TA (Equity to Total Assets) \\
PFN/EBITDA (Net Debt to EBITDA) \\
IE_CASHFLOW (Interest Expenses to Cash \\
Flow) \\
DSCR (Debt Service Coverage Ratio)
\end{tabular}

FIN_MISMATCH (financial mismatch)

CASH_ST_DEBT_S (Current Assets to Short Term Debt)

CASH_TA (Cash to Total Assets)

RECEIVABLES_TURNOVER

Turnover Ratio) - $\boldsymbol{N L}$

PAYABLES_TURNOVER (Payable Turnover Ratio) - NL

LOG_ASSETS (Natural Logarithm of Total Assets)

SALES_GWT (Net Sales Growth) - $\boldsymbol{N} \boldsymbol{L}$

\section{Description}

Ratio between net sales and total assets. The asset turnover ratio is an efficiency ratio that measures a firm's ability to generate sales from its assets.

Ratio between economic value added and total assets. Operating profitability ratio that measures the firm's ability to generate value from its assets.

Operating profitability ratio that measures how much earnings the firm is generating before interest, taxes, depreciation, and amortization, as a percentage of revenue.

Measure of a firm's financial leverage, calculated by dividing its net liabilities by stockholders' equity.

Ratio between equity and total assets. Used to assess a company's financial leverage

Debt sustainability ratio gives an indication as to how long a firm would need to operate at its current level to pay off all its financial debt.

Ratio that indicates the enterprise's ability to pay interest from generated cash flow.

Ratio of debt sustainability that refers to the amount of cash flow available to pay interest expenses and annual principal payments on financial debt.

Ratio of the mismatch (difference) between short-term liabilities and short-term assets and total assets. Negative value of the ration (shortterm liabilities $>$ short-term assets) indicates that the firm has enough short-term assets to meet its short-term liabilities.

Liquidity ratio that measures a firm's ability to pay off short-term debt obligations with cash and cash equivalents.

Ratio between cash and liquid assets to total assets. It measures a firm's liquidity and how easily it can service debt and short-term liabilities if the need arises.

(Receivable Efficiency ratio that measures how efficiently a firm is using its assets. It measures the number of times over a given period (usually a year) that a firm collects its average accounts receivable.

Efficiency and liquidity ratio that measures how many times a firm pays its creditors over an accounting period.

Measures the size of the firm.

Measures a firm's growth in a specific year. It also measures the stability of a firm's performance.

\begin{tabular}{llll}
\hline Variable & & Description \\
\hline DG_CR_TOT (Drawn amount to Granted & $\begin{array}{l}\text { Financial flexibility ratio. It measures the } \\
\text { percentage of available credit that the firm is }\end{array}$ \\
Amount) $-\boldsymbol{N L}$ & & & \\
\hline
\end{tabular}


actually using. It refers to all the different types of loans.

DG_CR_REV (Drawn Amount to Granted Financial flexibility ratio. It measures the Amount of uncommitted short term loans) - $N \boldsymbol{L}$ percentage of uncommitted short-term loans that the firm is actually using.

DG_CR_AUT (Drawn Amount/Granted Financial flexibility ratio. It measures the

Amount, short term loans) - $N \boldsymbol{L}$

DUMMY_SCONF (Overdrawns) percentage of self-liquidating short-term loans that the firm is actually using.

Dummy equal to 1 if the firm has an overdrawn amount greater than the granted amount, and 0 otherwise.

DEF_STORIA_CRED (Deteriorated loans)

MORTGAGE (Mortgage)

Dummy equal to 1 if the firm has deteriorated loans, and 0 otherwise.

Dummy variable equal to 1 if long-term loans are more than 90 per cent of total loans. It is used to mitigate the impact on PD estimation of a high drawn/granted ratio which is physiological for mortgages.

DUMMY_REV

Dummy equal to 1 if the firm has uncommitted short-term loans, and 0 otherwise.

DUMMY_AUT

Dummy equal to 1 if the firm has short-term loans, and 0 otherwise.

\begin{tabular}{ll}
\hline Variable & Description \\
\hline AREA_CVD (geographical area) & $\begin{array}{l}\text { Dummy variables identifying the geographical } \\
\text { region where the firm operates (North-East, } \\
\text { North-West, Center, South and Islands). }\end{array}$ \\
ATECO_CVD (economic sector) & $\begin{array}{l}\text { Dummy variables identifying firms' economic } \\
\text { sector. } \\
\text { Dummy variables identifying firm size as } \\
\text { defined by the European Commission. }\end{array}$ \\
\hline
\end{tabular}




\section{Modelli di business e modelli manageriali della banca Dal rischio di business model al rischio strategico. Verso una revisione del framework dei rischi bancari?}

Maurizio Baravelli (Sapienza Università di Roma)

\section{Abstract}

Il paper riprende gli aspetti teorici che ho trattato nella prima parte del position paper AIFIRM-APB, Business Model e SREP: il ruolo del CRO e del CFO e che ho commentato nel Webinar del 9 luglio scorso. Partendo da un quadro definitorio, approfondisco il tema del rischio di modello di business e delle sue relazioni con il rischio strategico. E pongo la questione della revisione del framework dei rischi bancari. In particolare evidenzio come il rischio di modello di business e quello strategico dipendano dal modello di management. Al tempo stesso, ampio spazio è dedicato a illustrare come il rischio di modello di management influenzi la sostenibilità del modello di business. Esamino le ricadute operative del quadro teorico del modello di business e propongo una revisione del processo di pianificazione aziendale. L'intento dell'articolo è quello di avviare un dibattito con l'intervento soprattutto degli specialisti di risk management.

The paper takes up the theoretical aspects that I dealt with in the first part of the AIFIRM-APB position paper, Business Model and SREP: the role of the CRO and the CFO and which I commented on in the Webinar of last July 9th. Starting from a defining framework, I deepen the theme of business model risk and its relationship with strategic risk. And I raise the question of revising the banking risk framework. In particular, I highlight how the business model and strategic risk depend on the management model. At the same time, ample space is dedicated to illustrating how the management model risk influences the sustainability of the business model. I examine the operational implications of the theoretical framework of the business model and propose a review of the business planning process. The purpose of the article is to start a debate with the intervention of risk management specialists above all.

\section{Premessa}

Mi è stato chiesto da alcuni amici di AIFIRM di ritornare sul tema degli aspetti teorici dei modelli di business (MB) che ho illustrato nel position paper AIFIRM-APB, Business Model e SREP:il ruolo del CRO e del CFO e che ho avuto modo di commentare nel Webinar del 9 luglio scorso, con particolare riferimento alla questione delle differenze tra MB e strategia anche al fine di chiarire come il rischio di $\mathrm{MB}(\mathrm{RMB})$ si rapporta al rischio strategico (RS).

Lo faccio molto volentieri per due motivi. Anzitutto perché ritengo che il MB sia un concetto, uno strumento e un framework, dipende da come vogliamo vederlo, che deve essere messo al centro del governo aziendale della banca perché aumenta l'efficacia delle prassi manageriali e può generare anche nuovi modelli direzionali. Secondariamente perché la questione del RMB è certamente intrigante nel senso che occorre comprenderne anzitutto l'utilità sia nel definirlo sia nel misurarlo. Nella letteratura però il concetto di RMB, pur essendo menzionato, non è affrontato sul piano operativo né la regolamentazione si è preoccupata di fornirne una definizione mentre definisce il RS. Ed è curioso che il regolatore si preoccupi di definire il RS e non definisca il RMB visto che la Business Model Analysis (BMA) riguarda sia la strategia sia il MB. I casi sono due: a) il RMB è considerato non rilevante; b) i due rischi- RS e RMB- si presuppongono coincidenti. Ma vi è in realtà una terza ipotesi: c) non dando una definizione di MB il regolatore si trova ovviamente nell'impossibilità di dire in cosa consiste il relativo rischio. Quindi la questione è da questo punto di vista "sospesa" essendo di natura teorica. Credo quindi utile ritornare all'inquadramento concettuale del MB di cui mi sono occupato nel position paper (Parte 1- Modelli di business: definizioni e componenti. Dalla letteratura aziendale e quella bancaria, pp.10-66) per riproporre i termini del problema della definizione di RMB e RS e delle loro relazioni fornendone una possibile interpretazione.

Si tratta nondimeno di una questione anche e soprattutto pratica perché sapere se il RMB è un argomento rilevante o meno interessa evidentemente la funzione sia di pianificazione sia di risk management che si trovano a dover prendere una posizione nell'ambito della loro attività a supporto delle decisioni aziendali. In altri termini le banche corrono un RMB senza valutarlo perché non se ne ha una definizione? In questo caso si tratta di un paradosso che deve essere superato. Non può rimanere infatti un argomento indeterminato, in attesa che si trovi una risposta che dovrebbe arrivare, a mio parere, anzitutto dal regolatore. In realtà il nostro position paper AIFIRM-APB si è proposto proprio di sviluppare un dibattito su questo aspetto per cui è opportuno ritornare a farvi riferimento anche perché una risposta da parte del regolatore non è ancora arrivata indipendentemente dall'analisi e dalle conclusioni del position paper. La discussione dovrebbe riguardare anche i tecnici e non solo i teorici. Un confronto tra teoria e pratica credo sia necessario per definire un quadro di riferimento che possa fare chiarezza anche sul piano operativo.

Nel position paper ho cercato di mostrare la centralità del MB nel governo della banca nel senso che il concetto di MB, la sua strumentazione, le metodiche connesse (cioè le relative pratiche manageriali) dovrebbero essere acquisiti dalla cultura manageriale e aziendale della banca. In altri termini il riferimento al MB non dovrebbe essere riservato solo a fini di vigilanza. Ho anche mostrato come la strategia può rapportarsi al MB tenendo separati e indipendenti i due concetti. In effetti esiste una certa confusione in letteratura e nella pratica perché vengono sovrapposti. E ho anche sottolineato a tal fine, come del resto è stato fatto in altre parti del position paper, l'integrazione auspicabile a livello operativo tra la visione del MB, della strategia e del risk management in un'ottica di collaborazione inter-funzionale, ma anche di confronto dialettico, nell'ambito dei processi aziendali di analisi, pianificazione e reporting. 
In questo contributo illustro la mia posizione, che non è in realtà solo mia, sulla questione delle relazioni tra MB e strategia: nella letteratura vi sono approcci secondo cui a livello sia teorico sia operativo i due concetti possono coesistere in un coerente quadro complessivo. Non è escluso però che non emergano ulteriori aspetti problematici e di dibattito come spesso accade quando gli argomenti vengono approfonditi. La chiarezza deve andare comunque a vantaggio di finalità pratiche che sono quelle che in questa sede vorrei sostanzialmente privilegiare.

Occorre anzitutto ricordare che la difficoltà di far diventare centrale il MB nel governo della banca, e mi riferisco anche alle implicazioni a livello di consiglio di amministrazione, dipende dal fatto che il concetto di strategia e quello di piano strategico si sono da tempo consolidati nel settore bancario. Infatti solo recentemente le banche sono state sollecitate dalla regolamentazione ad avvicinarsi al concetto di MB come strumento di analisi e diagnosi della propria sostenibilità economica. In passato la BMA non è stata una pratica manageriale delle banche e dei gruppi bancari italiani sebbene in sostanza nei piani strategici si facesse riferimento alle componenti dei MB seguendo il paradigma ASOP (cioè delle relazioni "ambiente-strategia-struttura-performance") utilizzato dalla Vigilanza della Banca d'Italia dagli anni Novanta. Si osservi pertanto come vi siano strette relazioni tra cultura manageriale e uso del concetto di MB. Se con la BMA si può parlare di innovazione della regolamentazione, si può parlare anche di innovazione manageriale nel momento in cui il concetto di MB e le pratiche connesse vengono adottati in modo sistematico dalla banca come strumento gestionale e non come un obbligo procedurale quando la banca viene sottoposta ai controlli di vigilanza.

La riflessione che propongo è articolata come segue. Dopo aver richiamato la questione delle relazioni tra MB e strategia, spiego le ragioni per le quali il MB dovrebbe stare al centro del governo aziendale. Mostro di conseguenza come tra MB e strategia vi siano relazioni di complementarietà e quali sono, in quest'accezione, le ricadute sulla pianificazione aziendale. Da questa analisi passo a porre la questione della definizione del RMB e del RS e mi soffermo sulle determinanti del RMB e sul ruolo del modello manageriale (MM) nel suo monitoraggio. Chiarisco quindi che esiste un rischio di MM (RMM) che si riflette sul RMB e sul RS ed evidenzio la necessità che a livello aziendale sia data particolare attenzione alle componenti di natura organizzativa del MB di cui il MM è una componente rilevante. Nelle conclusioni metto l'accento sul ruolo della ricerca e ritorno al quesito iniziale se il quadro normativo non debba essere rivisto per fare maggiore chiarezza sui concetti di MB e di strategia e sui rispettivi rischi.

\section{Relazioni tra modelli di business e strategia: coordinate di una querelle}

Credo che per avviare una discussione sulle relazioni tra il quadro teorico e quello normativo, ci si debba anzitutto interrogare su come la regolamentazione sia influenzata dalla letteratura, dall'esperienza dello stesso regolatore e da altre circostanze. Non dando una definizione né di MB né di RMB, a maggior ragione nell'ambito dello SREP, la regolamentazione, a mio parere, non si è voluta prendere l'onere di una revisione complessiva della tassonomia dei rischi bancari né la responsabilità di affrontare questioni dottrinali. Infatti il RMB potrebbe intendersi in diversi modi, come rischio specifico o come sommatoria di tutti i rischi della banca che riguardano quel MB. Di Antonio (2020) pone per esempio giustamente la domanda se il rischio di credito sia solo un rischio caratteristico o anche un rischio di tipo strategico o di MB. Di conseguenza entrare nel dettaglio su questi aspetti comporta la messa in discussione e la revisione del framework complessivo del sistema dei rischi bancari mentre il regolatore mi pare abbia seguito un principio cumulativo dei vari tipi di rischio, introducendone dei nuovi senza rivedere quelli precedenti. E' evidente che se si accetta la centralità del MB nel governo della banca, il primo rischio da definire e valutare dovrebbe essere quello di MB.

Come abbiamo detto, il RMB non viene definito dalla regolamentazione ma lo si può desumere dalla stessa quando fa intendere che la valutazione del MB tende ad accertare se questo è viabile nel breve e sostenibile nel medio. Ciò significa che le condizioni di viabilità e sostenibilità possono non esservi, avere livelli di adeguatezza nel tempo variabili e venir a mancare anche con il manifestarsi di perdite. In altri termini, la banca corre un RMB quando viene meno la capacità di mantenere livelli accettabili di profittabilità. Ora le cause di performance inadeguate, della loro variabilità o di perdite sono molteplici per le numerose variabili che influenzano le stesse performance del MB per cui se individuare il RMB è questione certamente complessa resta pure sempre di grande di rilievo.

Bisogna al riguardo considerare che l'uso concreto del concetto di MB a livello aziendale, essendo elaborato dalla letteratura, non è uniforme, risente delle interpretazioni pratiche e quindi è normale che vi possa essere una certa confusione. Inoltre il regolatore non dà una definizione di RMB ma si è limitato a darla del RS. Ciò può sorprendere perché anche nel caso della strategia le definizioni non sono univoche nella letteratura. Credo che il regolatore si sia trovato e si trovi in difficoltà a fare scelte definitorie in particolare del RMB in un tale contesto avendo già definito il RS per cui alla fine, forse inconsapevolmente, ha lasciato un "vuoto" non preoccupandosi di dare (o di ritornare a dare) una definizione né di MB né di strategia ritenendole "scontate" e note ma ciò non corrisponde alla realtà per quanto abbiamo detto. $\mathrm{O}$ meglio ciascuno resta con la propria nozione che preferisce o dell'idea che si è fatta. Ma proprio perché le interpretazioni di MB e di strategia sono molteplici il regolatore dovrebbe invece assumere a maggior ragione una posizione, chiarendo anche le relazioni tra i due concetti dal proprio punto di vista, e quindi dare le proprie definizioni e giustificarle a fini normativi.

La domanda-chiave che dobbiamo porci è pertanto se al centro di un più chiaro quadro normativo debba esservi la definizione del concetto di MB e del suo rischio. Per quanto sopra detto a me pare che una rilettura-revisione complessiva del quadro normativo non sia da ritenersi una "questione eccessiva" ma del tutto necessaria al fine di una maggiore chiarezza dal punto di vista delle definizioni delle varie categorie di rischio. Soprattutto per eliminare sovrapposizioni, ambiguità ma anche confusioni e quindi per stabilire una gerarchia dei rischi visto che alcuni possono essere interpretati come contenitori di altri.

Devo dire al riguardo che la regolamentazione si è comunque sempre mantenuta nei framework dottrinali della gestione e organizzazione della banca e più in generale dell'economia, strategia e organizzazione aziendale mutuandone concetti e paradigmi e adattandoli alle esigenze della supervisione. Ma questo passaggio dalla teoria alla normativa è avvenuto non sempre senza talune 
ambiguità a motivo dell'interpretazione dei paradigmi dottrinali, la difficoltà di tradurli in strumenti operativi e di controllo, ma anche delle posizioni contradditorie che si riscontrano nella stessa letteratura aziendale e manageriale. E questo spiega anche perché la normativa non dia una definizione "ufficiale" di MB.

Venendo ora al tema della distinzione tra strategia e MB e delle loro relazioni e di conseguenza alla querelle, chiamiamola così, riguardante l'utilità o meno di definire il RMB perché sarebbe simile a quello di RS, oppure perché è più rilevante il RS, dobbiamo prendere atto che una certa confusione era già preesistente almeno in parte prima dell'introduzione del concetto di MB. La questione si è complicata nel momento in cui, una volta accettata l'utilità del concetto di MB, si è cercato di stabilire quali relazioni possono esservi tra il $\mathrm{MB}$ e la strategia e di integrarli in una visione unitaria.

Come ho già detto nel position paper, le razionalizzazioni e sistematizzazioni concettuali di strategia e di MB incontrano dei limiti perché tali concetti sono nati e si sono sviluppati sulla base di presupposti e paradigmi differenti, in tempi e circostanze diversi. Il concetto di strategia nasce negli anni Sessanta del secolo scorso mentre si è cominciato a parlare di MB solo con lo sviluppo di Internet. Ciò nonostante, si è cercato di combinarli in vari modi, essendovi tra i due molteplici punti di contatto, ma con risultati non sempre convincenti, tra cui la conclusione che strategia e MB siano addirittura la stessa cosa.

Le differenze però restano: basti pensare che quando si parla di strategia l'enfasi viene posta soprattutto sulle modalità e capacità di competere dell'impresa mentre quando si parla di MB l'attenzione è posta sulle modalità e capacità di creare valore. Così un'impresa può decidere azioni per essere più competitiva ma può penalizzare il suo conto economico. Nel concetto di MB si insiste invece sulle caratteristiche strutturali e funzionali dell'impresa da cui dipende la creazione di valore, in particolare sulle combinazioni tra componenti tangibili e intangibili, compresi l'assetto organizzativo e il MM. La visione è quindi olistica e sistemica dell'assetto e del funzionamento aziendale e l'accento non è solo su un particolare punto di vista come quello delle modalità competitive ma si considera il sistema aziendale nel suo equilibrio complessivo cioè nelle sue relazioni/interrelazioni sia esterne che interne.

La strategia, per contro, interessa le interazioni esistenti o desiderate tra l'impresa e il proprio ambiente di riferimento, e deriva da un insieme di decisioni, di scelte e azioni dell'impresa per raggiungere i propri fini. Si tratta di decisioni e scelte - quelle strategiche a differenza di quelle tattiche - con basso grado di reversibilità per cui l'impresa incontra vincoli di tempo/costo per seguire nuove direttrici. L'interazione con l'ambiente muta nel tempo al variare delle caratteristiche ambientali. Interagendo con l'ambiente, l'impresa deve decidere in quali business operare, come competere, con quali mezzi e risorse. Il concetto di strategia può essere quindi più o meno ampio, andando dalla definizione del posizionamento di mercato fino a identificare l'identità complessiva dell'impresa. Da quest' ultimo punto di vista il concetto di strategia tende a sovrapporsi a quello di MB.

Vista come insieme di decisioni si distinguono: la strategia a livello corporate, che riguarda le scelte di portafoglio con effetti sull'equilibrio economico-finanziario complessivo quando l'impresa opera simultaneamente su più mercati; la strategia competitiva a livello di singola area di affari, cioè il modello di condotta nella gestione di una specifica combinazione prodotto/mercato/tecnologia; la strategia di funzione che definisce le politiche aziendali in ogni area funzionale, dall'organizzazione, alla finanza, al marketing e via dicendo in un'ottica di integrazione con le altre componenti dell'impresa. In questo senso la strategia è l'insieme coordinato dei fini, degli obiettivi e delle politiche aziendali. Resta comunque sempre prevalente il focus sul rapporto con l'ambiente, il mercato/i mercati di riferimento per cui la strategia evoca problemi di coerenza, reazione, adattamento alle caratteristiche e alla dinamica delle variabili esterne all'impresa (economiche, competitive, sociali, istituzionali, tecnologiche) al fine di conseguire le finalità e gli obiettivi perseguiti e mantenere condizioni adeguate/soddisfacenti di equilibrio economico, finanziario e patrimoniale.

Il concetto di MB è centrato invece sulla visione globale e unitaria delle modalità con cui l'impresa crea valore per se stessa e per il mercato/l'ambiente (cioè nei confronti dei vari stakeholder) attraverso il coordinamento di un insieme di componenti, tangibili e intangibili, strutturali e di funzionamento che configurano il modello imprenditoriale. Quindi l'aspetto competitivo e le relazioni tra l'impresa e il mercato spiegano solo in parte le performance dell'impresa stessa che invece l'assetto organizzativo e il MM e le relative modalità di funzionamento sono in grado di chiarire in un'ottica sistemica in cui le varie componenti interagiscono fra di loro. La pratica aziendale ha recepito il MB quale rappresentazione della struttura e del funzionamento aziendale e come strumento manageriale per la sua ideazione e il suo monitoraggio ma esso è diventato al tempo stesso anche strumento di ricerca per effettuare confronti interaziendali con l'obiettivo di comprendere come imprese dello stesso settore producano valore con il proprio "modello".

In questa prospettiva ogni impresa opera e compete in una o più "area di business" con un proprio MB producendo valore per il mercato e per se stessa. Pertanto è il corretto equilibrio tra valore esterno e valore interno, che l'impresa contestualmente crea, a determinare la sostenibilità del suo MB. Nonostante la questione delle interconnessioni tra le componenti del MB sia determinante per la comprensione del suo funzionamento, cioè di "come" crea valore, la letteratura si è maggiormente concentrata sulla questione della scelta delle componenti con cui identificarlo. Le definizioni di MB si sono nel tempo arricchite per meglio qualificarlo così alle definizioni sintetiche, con 1' esplicitazione delle componenti basiche, cioè di quelle ritenute astrattamente essenziali, si sono aggiunte definizioni molto articolate e dettagliate. Le principali "componenti di base" riguardano: la customer value proposition, i segmenti target, i prodotti, l'organizzazione e le risorse, gli aspetti economici e finanziari. A queste se ne trovano aggiunte altre come la partecipazione dell'impresa a network, la tecnologia, i processi - chiave, le competenze e le capacità manageriali. Si tratta comunque in buona parte di specificazioni delle componenti basiche. A livello pratico ogni impresa dovrebbe adottare una definizione di MB con un grado di dettaglio coerente con la sua specificità e il suo settore e soprattutto con gli elementi della business idea da cui deriva il MB. 
Quindi il RMB rispetto al RS, che guarda alle relazioni dell'impresa più sull'esterno che non sull'interno, dovrebbe contenere il "rischio organizzativo" (rischio di inadeguatezze dell'organizzazione), nelle sue varie componenti, e in particolare come chiarisco nel position paper, il RMM. Del MM la letteratura sui MB parla poco limitandosi a richiamarne alcuni profili tra cui le capacità e competenze manageriali, alle quali accenna anche la BMA dello SREP. Il MM è una componente importante del MB e del suo assetto organizzativo perché lo fa funzionare in modo più o meno efficiente ed efficace. Quindi il RMB non dipende solo dal sorgere di incoerenze nei confronti dell'ambiente esterno ma anche dal sorgere di incoerenze tra i fattori interni e dalla qualità del MM. Queste incoerenze /inadeguatezze del MM possono compromettere le condizioni di sostenibilità dello stesso MB anche senza variazioni del contesto esterno.

La domanda-chiave a cui occorre rispondere, alla luce di queste considerazioni, è se al centro di un più chiaro quadro normativo debba esservi messo il concetto di MB e tutto il sistema dei rischi debba ruotarvi attorno compresa la strategia e il RS con gli aggiustamenti che si rendono necessari. In questo contributo propongo una serie di riflessioni su questa questione. Ritengo che una rilettura complessiva del quadro normativo, che ristabilisca una maggiore chiarezza, sia necessario per eliminare sovrapposizioni, ambiguità ma anche confusioni, e per stabilire una possibile gerarchia dei rischi visto che alcuni sono interpretabili come contenitori di altri.

\section{Centralità del modello di business nel governo aziendale}

Per uscire dal vicolo cieco di conciliare i concetti esistenti (nella letteratura e nella pratica), non sempre conciliabili, in un quadro di coerenze complessive, difficile quindi da realizzare, si può ragionare ex novo mettendo al centro del governo aziendale il MB. E si può ragionare sulla gestione del $\mathrm{MB}$ nelle varie fasi che riguardano, potremmo dire, il suo ciclo di vita. Questa impostazione consente di reinterpretare il concetto di strategia ma in subordine al $\mathrm{MB}$ e in ogni caso può essere stimolante per gli obiettivi di questo contributo che intende procedere secondo un approccio tra il problematico e il propositivo in modo da alimentare una discussione.

Anzitutto occorre considerare che vi è sempre un momento di (1) ideazione e progettazione del MB. Alla base del MB vi è infatti una business idea la quale può derivare da fattori istituzionali come la missione e i fini statutari dell'impresa che prevedono che questa operi in un certo business, con certe modalità e finalità; ma può nascere da un'intuizione dell'imprenditore o dalla imprenditorialità diffusa che lo stesso imprenditore o la direzione dell'impresa promuovono nell'organizzazione; oppure, da un'analisi formale nell'ambito di processi di pianificazione; oppure da processi di learning organization oppure, ancora, da condotte imitative.

Dall'idea (formalizzata o non formalizzata) si passa alla progettazione della configurazione del MB nelle sue componenti tangibili e intangibili, strutturali e di funzionamento tali per cui si prevede che nel loro insieme interagendo siano in grado di creare valore sia esterno che interno. La progettazione dà luogo a un "disegno di MB" descrittivo, con schematizzazioni dell'assetto e del funzionamento organizzativo, compreso il MM, con l'indicazione di tutte le coerenze necessarie, e con proiezioni economicofinanziarie che assicurino condizioni di stabilità e sostenibilità. Questa fase, come le altre, può essere centralizzata o partecipata; questo aspetto di processo è importante e ne riparleremo più avanti.

Dall'ideazione-progettazione si passa alla fase di (2) implementazione del progetto di $M B$, il che significa approntamento concreto di tutte le componenti già disponibili e/o che devono essere acquisite e il loro coordinamento secondo il disegno progettuale. Il passaggio dalla fase 1 alla fase 2 non è immediato e lineare perché nella fase attuativa del MB ci si può rendere conto che le variabili definite in astratto devono confrontarsi con quelle reali ma anche che le relazioni tra le variabili intangibili e quelle tangibilifinanziarie non funzionano così esattamente come sono state immaginate. Queste relazioni nella fase progettuale sono infatti prefigurate e si presuppongono ma nella realtà non è detto che si verifichino e svolgano come sono state idealizzate. La fase attuativa coincide pertanto con una fase di sperimentazione-apprendimento per cui il MB viene perfezionato o addirittura modificato.

Questa fase di aggiustamenti-adattamenti-cambiamenti che possiamo chiamare fase di (3) monitoraggio del MB di fatto continua in tutta la vita del MB. Operando l'impresa ( e la banca) in un contesto dinamico, al fine di mantenere la creazione di valore su livelli adeguati (soddisfacenti) sia per la remunerazione del capitale sia per le attese della clientela e degli altri stakeholder o per altri fini istituzionali, il MB deve essere continuamente monitorato sia adattandolo ai cambiamenti del contesto esterno agendo sulle variabili (interne) del MB e sulla sua logica di funzionamento sia correggendo ed eliminando i fenomeni disfunzionali interni che possono sorgere indipendentemente dalla dinamica dei fattori esterni. Nel monitoraggio svolge un ruolo critico il MM che è quindi una componente determinante dell'assetto e del funzionamento del MB e della sua coerenza esterna ed interna. Sul MM ritorneremo essendo al centro di questo contributo. Come ho illustrato nel position paper, il MM riguarda l'assetto e la condotta del team direzionale, le sue competenze e capacità, le relazioni interne, la leadership, il grado di accentramento/decentramento decisionale, il coinvolgimento del personale. Queste variabili, ed altre ancora, come i sistemi manageriali, tra cui la stessa BMA e il concetto di $\mathrm{MB}$, sono il cuore del funzionamento efficace ed efficiente del MB.

L'importanza della fase del monitoraggio continuo deriva anche dal fatto che questa fase tende a riassumere le attività di analisi, riprogettazione e implementazione "senza soluzione di continuità" grazie a un apprendimento aziendale continuo basato sull'esperienza e l'andamento dei risultati. Ciò rende poco realistico l'approccio della progettazione-attuazione. Nella fase dell'attuazione possono rendersi necessarie correzioni nei confronti dell'esterno e/o dell'interno ma possono emergere anche nuove idee di business e l'opportunità di cambiamenti immediati del modello deliberato. Vi possono essere comunque scansioni all'interno del monitoraggio continuo qualora l'impresa preveda sottofasi formali distinte di analisi del MB corrente e dei suoi punti di forza e di debolezza, di definizione di un piano conseguente di revisione con l'indicazione dei cambiamenti necessari e di un piano di implementazione di tali cambiamenti. E' interessante al riguardo osservare che la letteratura sui MB è concentrata sulla fase di 
analisi e progettazione, che comprende la revisione e l'innovazione, ma non affronta la fase della implementazione. Ma se si parla di progettazione del MB si dovrebbe parlare anche della problematica di tutte le altre fasi. Infatti il monitoraggio può portare l'impresa a innovare il MB oppure a migrare verso altri MB. La fig.1 schematizza le possibili evoluzioni.

Figura 1 - Pianificazione e ciclo di vita del modello di business

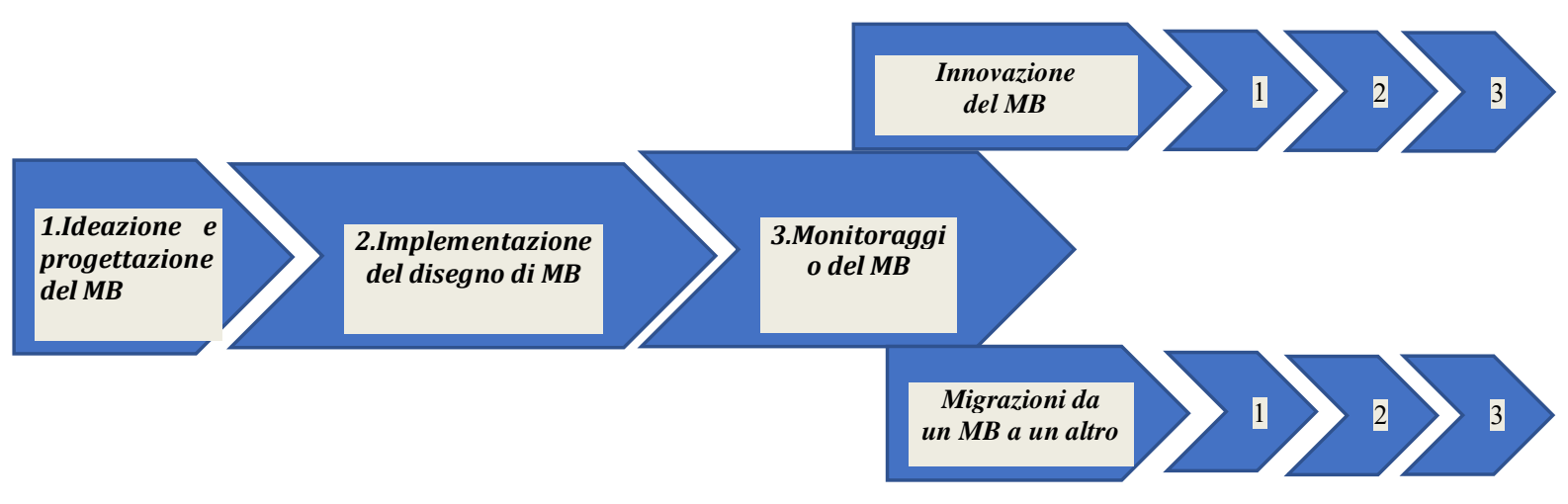

Questo approccio al governo del MB, che riteniamo vada messo al centro delle pratiche manageriali, non è sviluppato dalla letteratura che quando parla di pianificazione lo fa con riferimento alla strategia ma la questione è stata comunque sollevata (Osterwalder et al. 2005): se si parla di definizione del MB si dovrebbe parlare anche del tema della sua implementazione. Tuttavia mentre, come detto, la letteratura si è concentrata sugli aspetti metodologici della definizione, scarso rilievo ha dato alla questione dell'implementazione ritenendola scontata. L' implementazione non è però questione secondaria perché presenta proprie problematiche; infatti è la fase realizzativa quella che conta perché solo quello attuato è il MB effettivo dell'impresa che può differire pertanto da quello deliberato. C'è infatti differenza fra il disegno di un $\mathrm{MB}$ e la sua effettiva implementazione: un MB ben progettato può essere mal realizzato, così come un MB mal progettato e con difetti può trovare nella fase realizzativa soluzioni di miglioramento o essere modificato perché risulta non realizzabile oppure risulta diverso per altri motivi, per errori o scasa efficacia attuativa.

Ma se si accetta il concetto di pianificazione del MB quali sono le relazioni con la pianificazione della strategia? Si tratta dello stesso processo? Nelle pagine seguenti cercheremo di dare una risposta a queste domande mostrando come sia possibile conciliare il concetto di $\mathrm{MB}$ con quello di strategia. Come vedremo ne deriva anche la possibilità di costruire un nuovo framework della pianificazione aziendale che può fornire un'interpretazione nel caso delle banche di come la MBA dello SREP pare intenda le relazioni tra MB e strategia. Il regolatore fa riferimento sia al MB sia alla strategia ma lascia il quadro normativo lacunoso e da interpretare non dando una definizione né di MB né di strategia né tanto meno specificando le loro relazioni.

\section{Relazioni "sequenziali" tra modello di business e strategia: ricadute sul piano operativo}

Con riguardo alle domande precedenti, si osservi come il tema delle relazioni tra MB e strategia sia stato affrontato dalla letteratura e una risposta possa essere ricercata tra gli autori che si sono interrogati, come Seddon e Lewis (2003), se sul piano astratto-deduttivo venga prima la strategia o il MB. Porsi in effetti la questione su "which comes first: strategy or business model" è utile per fare chiarezza su come i due concetti possono essere utilizzati in pratica. In effetti molti sono i fattori comuni dovuti anche al fatto che nel definire il MB si utilizzano elementi elaborati e studiati dallo strategic management. Come si vede nella fig. 2 la questione sembra però irrisolta perché le ipotesi di relazione sono molteplici: vanno da più o meno ampie intersezioni a considerare i due concetti equipollenti, alla assunzione che la strategia contenga il MB o che questo contenga la strategia.

Volendo esprimere il nostro pensiero riteniamo che il concetto di MB sia più ampio di quello di strategia perché quest'ultimo è focalizzato sul lato esterno delle relazioni con il mercato e sui fattori competitivi e non enfatizza i profili organizzativi come invece si osserva nel concetto di MB. Questo richiama un quadro completo delle componenti non sono tangibili ma anche intangibili. Del resto nelle definizioni di MB ricorre spesso il richiamo specifico alla strategia.

Ciò premesso, la strategia, coerentemente con quanto abbiamo detto sulla centralità del MB e sulla pianificazione del MB, va collocata nel nuovo framework concettuale e quindi occorre reinterpretarne il ruolo. Nel position paper ho proposto come sia più convincente e coerente la tesi di Casadesus-Masanell e Ricart (2010) secondo cui il concetto di MB e quello di strategia sono distinti e diversi ma complementari nel senso che la strategia può essere vista come lo strumento del monitoraggio del MB e quindi funzionale al governo del MB. 

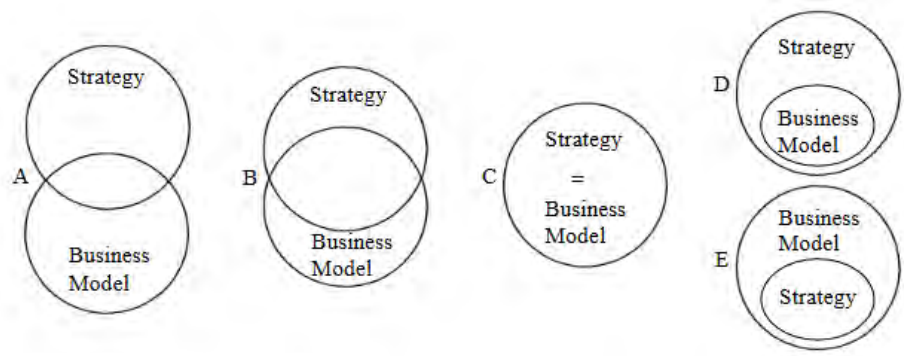

Fonte: Seddon e Lewis (2003)

Secondo tale approccio, la pianificazione del MB indica se e come il MB corrente deve essere modificato prefigurando il MB a tendere mentre la strategia (piano strategico) stabilisce gli obiettivi da perseguire e le azioni necessarie per realizzare le suddette modificazioni e il MB a tendere. Questo modo di ragionare cambia la "narrazione" sul ruolo della strategia perché si deve parlare anzitutto di definizione, adattamento, revisione del $M B$. Di strategia si può parlare di conseguenza a proposito delle azioni di cambiamento "al margine" del MB, cioè delle sue correzioni e modificazioni. Poiché il MB non necessariamente deve essere modificato in tutte le sue componenti, la strategia con coincide con il MB; in ogni caso resta il fatto che un conto è definire il MB da costruire (il disegno di MB) un conto è definire un piano da seguire per la sua realizzazione.

Per esempio se la banca decide di entrare in un nuovo mercato, si tratta di stabilire quali attività promozionali attuare e la loro tempificazione, come modificare eventualmente i canali distributivi che devono essere adeguati ai nuovi segmenti di mercato, quali capacità e competenze occorre avere, come controbattere le possibili reazioni della concorrenza. Le modificazioni da apportare al MB corrente, nella loro visione complessiva e schematica, mostrano come sono in grado di creare valore /o di rendere meglio sostenibile il MB ma implicano delle politiche aziendali per consentire il passaggio dal MB attuale al nuovo MB. A meno che la definizione del MB a cui tendere non sia cosi dettagliata da configurare anche un piano per realizzarlo. Pertanto, se vogliamo combinare il concetto di MB con quello di strategia, mantenendo i due concetti distinti, l'apparato concettuale strategico risulta funzionale a pianificare come realizzare le variazioni e modificazioni del MB nel suo ciclo di vita. In altri termini, ciò significa che tra MB e strategia vi sono relazioni di tipo sequenziale (fig.2).

Pertanto è la strategia che adatta il $\mathrm{MB}$ alle continue dinamiche del contesto ambientale e competitivo con modificazioni più o meno complesse e incisive. In questa visione la strategia viene considerata nel suo concetto più ampio riguardante anche le modificazioni dei fattori organizzativi quando il MB richiede variazioni del modello strutturale e del MM. In altri termini, la strategia è diretta a rivedere il MB nelle sue componenti e relazioni interne/esterne anche se il MB viene mantenuto con le sue caratteristiche identitarie. Da questo punto di vista il MB si può interpretare come l'effetto, il risultato, l'output dell'azione strategica. Quanto alla generazione di MB innovativi (e di nuove business idea) questi non necessariamente possono emergere dal processo di pianificazione aziendale che però li può prendere in considerazione verficandone la fattibilità strategica.

Fig. 3 - Relazioni sequenziali tra modello di business e strategia

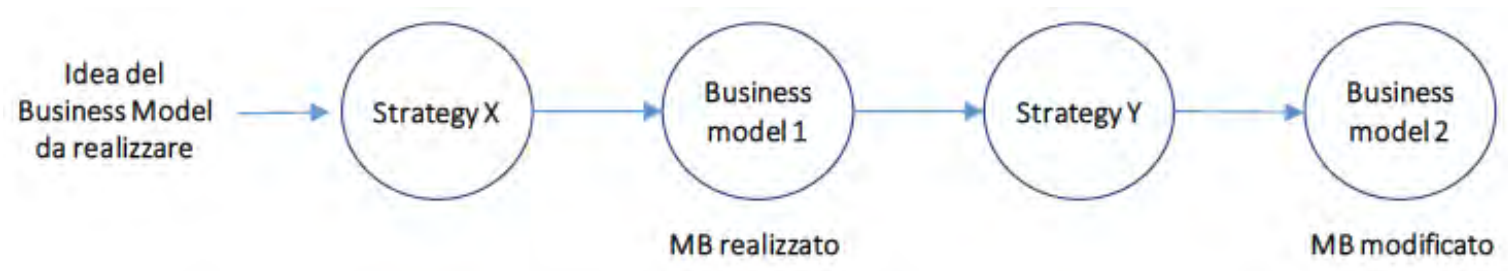

Fonte: Baravelli (2020a)

Questo approccio, come dicevamo, ha importanti ricadute pratiche. Infatti consente di pensare a un nuovo framework della pianificazione e a nuovi modelli di reporting. La pianificazione si trova a incorporare come primo step l'analisi del MB corrente e la valutazione della sua sostenibilità prospettica. In tal modo il riferimento al MB non è confinato alle relazioni con la Vigilanza ma viene a costituire lo starting point della pianificazione aziendale da cui derivano le variazioni e i cambiamenti, se necessari, da attuare per renderlo sostenibile. Queste variazioni e cambiamenti sono indicati nel piano strategico. La BMA entra quindi a pieno titolo nel processo di pianificazione. In tal modo sono rese più esplicite, e meglio argomentate, le ragioni delle azioni da intraprendere al fine dello sviluppo e della sostenibilità del MB. Nel contesto di una dialettica inter-funzionale la MBA dovrà avvenire secondo un modello partecipativo con l'intervento della funzione di risk management ( su questo aspetto ritorneremo più avanti) che dovrà indicare in una sezione del piano la valutazione (a) del rischio del MB corrente senza alcuna modificazione; (b) il rischio della strategia che consente di passare dal MB corrente al nuovo $\mathrm{MB}$ e (c) il rischio del $\mathrm{MB}$ che risulta dal cambiamento strategico cioè del MB a tendere (secondo lo SREP nei tre anni successivi).

Dobbiamo subito precisare perché si può parlare sia di RMB sia di RS. Intanto la valutazione della sostenibilità del MB implica di per sé che il MB corrente possa avere un rischio di sostenibilità che dovrebbe essere quindi misurato. Se il rischio è basso o nullo, 
la banca potrebbe ritenere che non siano necessarie modificazioni per cui viene mantenuto l'assetto attuale. Se invece si valuta che le performance del MB corrente sono in prospettiva declinanti la banca può decidere di modificare il MB corrente ma nel piano aziendale si dovrà chiarire come il nuovo MB - poco o molto diverso da quello attuale - risulta sostenibile (o più sostenibile del precedente). Pertanto si tratta di valutare anche la sostenibilità del MB a cui tendere.

Sarebbe infatti un paradosso fare nuovi investimenti quando nel corso dei tre anni successivi lo scenario ipotizzato potrebbe modificarsi. Questo rischio si configura però in concreto solo quando il nuovo MB sarà esistente nelle sue componenti di struttura e di funzionamento. La sua realizzazione potrà richiedere poco o molto tempo a seconda dei cambiamenti da attuare. Il riferimento è quindi ai contenuti e alla complessità della strategia. Vi sono cambiamenti rapidi e cambiamenti complessi che richedono tempo come nel caso in cui vi siano di mezzo cambiamenti della cultura aziendale e l'entrata in una nuova area di affari. Pertanto il rischio del "MB in progress" si manterrà più o meno vicino a quello del $\mathrm{MB}$ corrente e potrà peggiorare o migliorare a seconda dell'andamento delle condizioni del contesto interno ed esterno a meno che la strategia non sia rapidamente implementata con successo.

Ma anche come realizzare i cambiamenti e le azioni previste per mantenere sostenibile il MB ha un suo rischio. La strategia come risulta definita (e ci riferiamo alla formalizzazione in un piano) potrebbe non risultare adeguata a realizzare il disegno più o meno schematico del nuovo MB. Qui potremmo dire di essere in presenza del rischio del MB a cui tendere. Ciò dipende in particolare dal grado di dettaglio dei cambiamenti da apportare alle componenti del MB corrente che vengono delegati alla strategia; ammesso che le correzioni/modificazioni/innovazioni necessarie siano correttamente previste dal piano potrebbero non essere così puntualmente attuabili. Potrebbe accadere inoltre che la strategia risulti troppo complessa e ambiziosa per essere effettivamente realizzata. Pertanto la banca potrebbe trovarsi in mezzo al guado tra il MB corrente e un nuovo MB incompleto. Inoltre nella fase implementativa potrebbero sorgere degli ostacoli e delle reazioni della concorrenza a cui la banca non sa prontamente reagire sebbene il MB a cui tendere sia in linea di principio sostenibile.

Si deve quindi convenire che il RMB non coincide necessariamente con il RS essendo il MB e la strategia due "oggetti" differenti e va preso atto che sono proprio le differenze di rischio di cui deve tenere conto il processo decisionale strategico. La banca si può trovare nella condizione di accettare il maggior rischio del $\mathrm{MB}$ corrente perché cambiare $\mathrm{MB}$ appare più rischioso dovendo la banca intraprendere una strategia complessa dall'esito incerto. Ma può anche accadere che il rischio implicito nel nuovo MB risulti inferiore al rischio di continuare a mantenere un $\mathrm{MB}$ corrente che sta diventando maturo. Il costo della migrazione risiede in buona misura nel rischio strategico.

Si osservi inoltre come il RS non coincida necessariamente con il rischio del nuovo MB quando la strategia comporta modifiche e cambiamenti parziali del MB corrente. Per stabilire il rischio del nuovo MB (effettivamente realizzato) occorre considerare come la strategia impatterà il $\mathrm{MB}$ corrente modificando il suo assetto complessivo. Quindi la strategia non rappresenta necessariamente il nuovo MB. Ma occorrerebbe anche stabilire quando si può parlare correttamente di strategia. Si osserva infatti come accanto a strategie che hanno un impatto significativo sul $\mathrm{MB}$, le cosiddette strategie radicali, vi siano semplici correzioni, più vicine al concetto di strategie di mantenimento o di condotte tattiche, che non modificano sostanzialmente il MB (Yip 2004). Se il MB corrente non è sostenibile è probabile che siano necessarie strategie di tipo radicale come quando l'evoluzione del contesto ambientale lo rende obsoleto.

Se si assume che MB e strategia siano due concetti complementari che presuppongono relazioni di sequenzialità, si può pensare di rivedere il processo di pianificazione aziendale rendendolo più articolato e inserendovi la valutazione sia del RS sia del RMB. In tal modo il piano strategico viene a essere integrato nella MBA (fig.3). Ciò costituirebbe un'importante evoluzione del sistema manageriale della banca visto che dai risultati della survey a supporto del position paper emerge che l'analisi del MB è vista da buona parte delle banche un obbligo della Vigilanza per cui valutazione del MB e piano strategico sono momenti e documenti tenuti distinti. Non è questa la sede per un'analisi di questo processo integrato, ciò che vogliamo evidenziare è che la logica della sequenzialità tra $\mathrm{MB}$ e strategia ha molte interessanti ricadute sul piano operativo. Pertanto la questione delle relazioni tra MB e strategia non sono rileva sul piano teorico ma appare essenziale per comprendere le ricadute del quadro concettuale sulle pratiche manageriali.

Fig.4 - Pianificazione aziendale: monitoraggio del modello di business e strategia

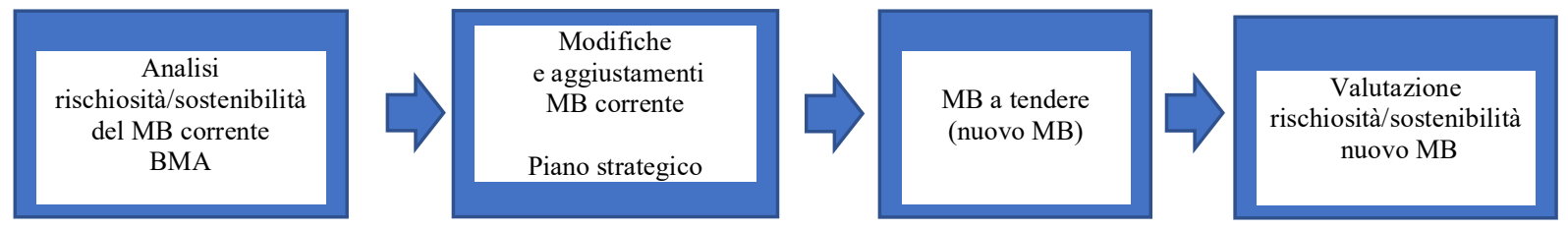




\section{Definizione di rischio di modello di business}

Quando si parla di modello imprenditoriale o modello di impresa, che è in sostanza l'equivalente di MB (nella versione in italiano della normativa a "business model" corrisponde "modello imprenditoriale"), si fa riferimento al generico rischio economico che l'imprenditore (la proprietà) corre di non vedere remunerato in modo adeguato il capitale investito. Questo rischio emerge sia nel momento dell'avvio dell'impresa sia durante tutte le fasi della sua vita, o meglio del suo ciclo di vita. Quindi tutti i MB corrono questo rischio, cioè che l'impresa non produca adeguati profitti, che l'impresa si trovi a operare in perdita fino al punto del suo fallimento.

Nel comprenderne le determinanti il rischio di impresa potrebbe essere associato al rischio di settore cioè al rischio del business in cui opera l'impresa. Questo può dipendere da andamenti avversi del mercato, da cambiamenti nelle preferenze dei consumatori, da nuovi concorrenti e da altri fattori che riducono i ricavi senza che l'impresa possa reagirvi con la riduzione dei costi (Di Antonio 2020). In questo caso sarebbe "quel business" a non essere remunerativo o a diventare non più remunerativo e vi sarebbero soggette tutte le imprese che vi operano. Cosa diversa è dare enfasi al concetto di "modello" perché in questo caso il rischio non riguarda un dato business in sé (cioè la sua attrattività economica) ma le modalità con cui l'impresa gestisce quel business. Di conseguenza sono le modalità di gestione del business che fanno la differenza nel determinare il RMB.

Infatti nell'ambito di una crisi di settore vi sono imprese in grado di reagirvi (evidenziando una capacità di reazione-risposta superiore mantenendo un normale o soddisfacente livello di redditività e limitando le perdite) mentre altre imprese soccombono. Più in generale, senza fare riferimento a scenari di crisi, è normale osservare performance diverse nell'ambito dello stesso settore/business. In questo caso il RMB può essere visto anche come un rischio competitivo cioè la possibilità che i concorrenti che operano nello stesso settore di attività sviluppino $\mathrm{MB}$ più efficienti ed efficaci.

Da queste considerazioni si ricava che il RMB dipende dalle modalità con cui l'impresa, e quindi anche la banca, gestisce/governa il business o i business (a seconda che sia monobusiness o multibusiness) in cui si trova a operare o decide di operare. Il focus sul modus operandi rimanda proprio al concetto teorico di MB, perché nella definizione più condivisa esso viene visto come un insieme di componenti (struttura) che interagendo (funzionamento) creano valore. Quindi entrano in gioco nel RMB sia le scelte strutturali sia i modelli di funzionamento. Ciò significa che per evitare il rischio di business, o limitarlo, la banca deve avere una capacità di reazione (possiamo dire superiore) nel modificare il proprio profilo sia strutturale sia di funzionamento. Ma il RMB può presentarsi anche in assenza di un rischio di business (crisi settoriale) a causa di fattori interni (concause) che pregiudicano l'efficiente funzionamento dell'impresa.

Infatti un $\mathrm{MB}$, regolarmente funzionante, a un certo punto può subire un calo di performance anche quando il contesto ambientale è relativamente stabile o comunque non vi sono cambiamenti di rilievo. Ciò accade per esempio per un cambio di management, per il sorgere di conflitti interni, per inadeguate politiche del personale, incentivi manageriali distorsivi, controlli che si sono indeboliti, comportamenti opportunistici, demotivazione del personale, leadership non più efficace. Questi fattori, e potremmo andare avanti nella loro elencazione, possono ridurre la produttività, indebolire la capacità competitiva (lasciando che i concorrenti si rafforzino indirettamente), far aumentare i costi operativi e i rischi tecnici.

Dietro a questi fenomeni vi sono sostanzialmente debolezze organizzative che deteriorano il MB. Siamo in presenza di un MM che diventa meno efficiente/efficace. Il MB subisce un effetto entropico dovuto alla perdita di energia organizzativa e, potremmo dire, di slancio emotivo dell'organizzazione. Il MM può essere pertanto esso stesso la causa della crisi del MB. In conclusione, il MB può vedere ridursi progressivamente le proprie performance non tanto e non solo per fattori esterni a cui l'impresa non sa reagire ma anche per circostanze e fattori interni, presenti in modo latente che a un certo punto emergono e che, puranche in condizioni di assenza di rischi settoriali, ne pregiudicano l'efficiente funzionamento.

Per esempio MM verticistici che puntano soprattutto alla riduzione dei costi e all'efficienza operativa generano assetti organizzativi di tipo meccanico-burocratico. Questi modelli hanno l'effetto di impoverire il capitale umano e creare contesti poco motivanti. L'evoluzione strategica è di conseguenza frenata da una minore capacità di adattamento alle dinamiche ambientali e di mercato nel momento in cui la banca si trova a dover puntare alla delega, al coinvolgimento e deve poter disporre di un personale motivato e qualificato. In questo caso, come in altri in cui le situazioni disfunzionali organizzative sono riconducibili al MM, si può parlare di RMM che si riflette a sua volta sul RMB. Se si condivide che il MM è una componente rilevante del MB e che il RMB è influenzato in modo determinante dal RMM, si dovrebbe concludere che RMM e RMB tendono a coincidere.

Ciò detto, se vogliamo ora confrontare il nostro approccio con il quadro normativo dobbiamo limitarci a considerare quanto il regolatore prevede in merito al RS perché non fornisce una definizione di MB né del relativo rischio. E' possibile però fare riferimento agli aspetti organizzativi. Secondo la normativa lo strategic risk è "il rischio attuale o prospettico di flessione degli utili o del capitale derivante da cambiamenti del contesto operativo o da decisioni aziendali errate, attuazione inadeguata di decisioni, scarsa reattività a variazioni del contesto competitivo" (Banca d'Italia, 2013, Titolo 3, Capitolo 1, Allegato A: traduzione italiana da CEBS 2006, oggi EBA). Prescindendo per un momento dal fatto che il RS può essere definito come incoerenze della strategia nei confronti dell' ambiente esterno senza precisarne le cause (che possono essere molteplici), nella definizione di strategic risk si citano quattro circostanze generali che hanno peraltro implicazioni con altri rischi : a) variazioni del contesto operativo; b) decisioni aziendali errate; c) attuazione inadeguata; d) scarsa reattività a variazioni del contesto competitivo.Queste circostanze sono tutte espressione di fattori organizzativi riferibili al MM. Al tempo stesso si può anche dire 
che il RS è mitigato dalla qualità del MM. Su questo punto si noti come l'importanza del MM sia sottolineata dalla stessa letteratura strategica che indica la flessibilità manageriale quale fattore di mitigazione del RS (Lorange,1980) sebbene l'argomento non sia approfondito.

Si potrebbe pertanto concludere che la regolamentazione nel definire il RS sottende un riferimento indiretto al RMB. In effetti il regolatore cita le variazioni del "contesto operativo", che è interno, e del "contesto competitivo", che è esterno, l'efficienza del processo decisionale (errate decisioni) e l'efficacia organizzativa e manageriale (attuazione inadeguata e scarsa reattività). In tal modo si può percepire una certa sovrapposizione tra $\mathrm{MB}$ e strategia e tra i relativi rischi. Il regolatore richiama le variazioni del contesto competitivo; ma la flessione degli utili e del capitale può dipendere anche da un calo di tensione organizzativa, dall' indebolimento della leadership o della qualità di altri variabili del MB. Questi aspetti si possono far rientrare nelle "variazioni del contesto operativo"? In effetti il decadimento dei fattori organizzativi e manageriali non è fenomeno poco frequente e non sono rari gli insuccessi delle strategie bancarie dipendenti da fattori endogeni e che si accentuano in presenza di variazioni dei fattori esogeni.

Se si condivide che MB e strategia non sono la stessa cosa perché tra il MB e la strategia vi è una relazione "sequenziale", il RS non può coincidere con il RMB. Il RS può essere riferito sia a un'errata definizione degli aggiustamenti da apportare al MB corrente per renderlo sostenibile (e traghettarlo verso il MB a tendere) sia a una errata attuazione di tali aggiustamenti sia a una errata attuazione del MB quando è progettato ex novo (fig.3). Queste cause sono in parte quelle citate dalla definizione di RS che ne dà la regolamentazione, ma il nostro approccio colloca il RS nel governo del MB. Si noti comunque come la normativa faccia riferimento indirettamente al MM sia quale causa del RS sia quale leva per mitigarlo.

\section{Rischio di modello di business: specifico o somma di rischi?}

Poiché ogni MB della banca ha suoi rischi caratteristici - credito, mercato, interesse, liquidità- il RMB deve essere visto come una sommatoria di rischi che confluiscono nel rischio economico di impresa? Oppure va considerato un rischio specifico? Non inten do qui entrare in una discussione tecnica, che lascio agli esperti di risk management, ma osservo semplicemente che se per evitare duplicazioni occorre tenere conto della distinzione fra rischi indipendenti e rischi correlati, sul piano concettuale è normale pensare che tutti i rischi debbano essere identificati e investigati anche quelli in qualche modo concatenati. Avere infatti un quadro chiaro e completo di tutte le forme in cui si manifestano è essenziale per intervenire sull'assetto e sul funzionamento del MB al fine di evitarli, mitigarli e gestirli nel modo migliore.

Se il RMB esprime il grado di sostenibilità del MB che può variare (molto basso, basso, alto, molto alto, crescente, decrescente, stabile, adeguato, inadeguato, e così via) in rapporto alla natura del business, al contesto esterno, al capitale investito e alle caratteristiche del suo MM ( che concorre a determinarlo da un lato e a mitigarlo dall'altro), credo che non lo si possa limitare alla capacità sopravvivenza del MB per cui per valutarlo si ci debba interrogare sul possibile rischio di disruption. Questa accezione molto specifica di RMB viene riferita alla possibilità che a causa di fattori esogeni il MB (e quindi la banca) possa entrare in una crisi irreversibile e sia così compromessa la sua continuità e sostenibilità. L'ipotesi è cioè quella di una banca che si venga a trovare di fronte a cambiamenti strutturali del contesto esterno, a radicali cambiamenti della regolamentazione, della tecnologia, della domanda della clientela, con la conseguente obsolescenza del proprio "modo di fare banca" (Di Antonio 2020). I cambiamenti esterni devono essere però così rapidi e radicali che la banca non riesca a fronteggiarli per tempo con riorganizzazioni, diversificazioni e migrazioni verso altri MB. Una seconda ipotesi sempre di rischio di collasso si può avere in seguito a un eccessivo grado di rischiosità complessiva dovuta alla somma dei vari rischi che strutturalmente caratterizzano il MB. In questo caso la sostenibilità del $\mathrm{MB}$ può essere tendenzialmente precaria per l'alto rischio assunto ma il $\mathrm{MB}$ può diventare insostenibile quando le scelte diventano eccessivamente rischiose come quelle speculative.

Che senso hanno queste concettualizzazioni di RMB? Riferendosi a casi limite illustrano situazioni in cui il MB non è più sostenibile mentre il RMB può essere graduato in rapporto a variazioni di fattori interni ed esterni che determinano variazioni delle performance aziendali. Il RMB può modificarsi e incrementarsi a seconda dell'andamento del ciclo congiunturale senza che si verifichino dinamiche esterne radicali che mettono in discussione la sopravvivenza della banca. Poiché il mantenimento di livelli soddisfacenti di profittabilità dipende pur sempre dalla capacità del MM di reagire al possibile decadimento delle performance, la valutazione del RMB è funzionale a comprendere soprattutto come reagire a tale eventualità e non tanto alla misurazione quantitativa del rischio stesso.

Ciò che quindi interessa, e ci riferiamo alla funzione di risk management, è poter disporre di un framework con cui valutare l'impatto di eventi, fattori e aspetti incerti sulle singole componenti del MB che possono influire sulla sostenibilità del MB e al tempo stesso verificare quali misure preventive predisporre o che sarà possibile adottare.Occorre quindi partire dalla rappresentazione del $\mathrm{MB}$ ed associare a ciascuna componente del MB i fattori di incertezza e i rischi che la riguardano (Brillinger et al. 2020) in modo da verificarne l'impatto sulla creazione di valore. Da questo punto di vista è determinante come la banca definisce il MB e il grado di dettaglio delle relative componenti.

La valutazione del RMB (e per converso la prospettiva di poter mantenere o meno un certo livello di profittabilità) dovrebbe qualificare il processo decisionale anzitutto della struttura di portafoglio delle attività della banca. Il rischio/rendimento prevedibile influenza l'attrattività del MB tenuto conto delle competenze e capacità del MM. Così se il RMB risulta in crescita la banca può decidere di dismettere quel $\mathrm{MB}$, di modificare la composizione delle linee di business e migrare verso altri modelli. Comunque anche nel caso di cambiamenti significativi esterni, non è escluso che la banca possa reagirvi con innovazioni grazie all'efficacia 
del proprio MM e ci riferiamo alle conoscenze, alla rapidità dei processi decisionali, alla leadership, alla capacità di coinvolgere le risorse. Nella valutazione del RMB è quindi determinante sempre la valutazione del RMM.

Come sta accadendo in questi ultimi tempi con la crisi pandemica del Covid-19, vi sono banche che hanno mostrato un'elevata flessibilità adottando meglio di altre e più rapidamente cambiamenti organizzativi e di prodotto, dando vita a nuovi modelli digitali sia nell'erogazione dei servizi nei confronti della clientela sia sul fronte delle procedure interne, delegando a gruppi di lavoro decisioni e autonomie organizzative e realizzando nuovi sistemi di lavoro. La maggiore capacità di risposta è riferibile a MM più flessibili soprattutto delle banche di non grandi dimensioni il cui contesto organizzativo favorisce, rispetto ai grandi gruppi, una maggiore coesione interna.

Il problema dell'adattamento della banca al contesto esterno è più generale e sta caratterizzando l'evoluzione dell'intero settore bancario alle prese con il rinnovamento del proprio core business costituito dall'attività creditizia tradizionale sempre meno attrattiva sul piano del rischio/rendimento. L'opzione più opportuna sarebbe quella di integrarla con attività creditizie e finanziarie a maggiore valore aggiunto rientranti per esempio nel corporate finance, nella finanza straordinaria e mobiliare. Si tratta di servizi poco presenti nei MB bancari ma essenziali in periodi come quelli che stiamo vivendo di crisi e recessione per sostenere le imprese in difficoltà ma anche necessari in fase di crescita per sostenerne lo sviluppo. Per di più in combinazione con il credito tradizionale essi costituiscono un'arma competitiva. Del resto il regolatore europeo è orientato a promuovere maggiormente i mercati e gli intermediari non bancari e quindi la concorrenza è destinata ad aumentare. Ma per andare in tale direzione le banche devono anzitutto modificare gli attuali MM investendo in nuove conoscenze e competenze e adottando stili direzionali basati sul knowledge management, promuovendo l'empowerment, valorizzando il middle management, e puntando a modelli organizzativi decentrati (Baravelli 2000b).

Le banche che non intraprendono questa evoluzione, specie quelle che fanno prevalentemente credito tradizionale, sono esposte verosimilmente al RMB. La crisi pandemica del Covid-19 sta accentuando il RMB di molte banche. Il regolatore ha consentito di operare al disotto dei ratio previsti dalla normativa attenuando l'impatto negativo della crisi ma facendo ritardare l'evoluzione necessaria con un peggioramento della situazione a medio temine quando i vincoli dovranno essere rispettati. Le banche si troveranno con un'ulteriore riduzione della redditività per l'aumento dei NPL e una clientela con rischi di default (Brogi 2020). Le banche dovrebbero quindi accettare un RS di innovazione e diversificazione perché può risultare maggiore il rischio di mantenere gli attuali MB.

È vero che l'incertezza rende difficile calcolare il RMB ma poiché esso incombe le banche devono essere pronte e aumentare la propria flessibilità e capacità di adattamento puntando sulla qualità del capitale umano e su MM meno gerarchici e più imprenditoriali. Infatti il principale vantaggio competitivo risiede nell'organizzazione e nel MM. Pertanto disporre di modelli organizzativi flessibili, che sappiano sperimentare e apprendere, è un punto di forza di chi è in grado cogliere tempestivamente le nuove opportunità emergenti. Quanto alle singole business unit di una banca diversificata il rischio di disruption può non essere così infrequente ma le conseguenze non è detto che siano tali da portarla al dissesto. La situazione è diversa se tale rischio riguarda il core business.

\section{Modello manageriale e monitoraggio del modello di business}

Con il monitoraggio la banca verifica l'andamento del grado di sostenibilità del proprio MB in rapporto agli scenari, alle performance conseguite e al contesto interno. Le modificazioni e i cambiamenti del MB che si rendono necessari, derivanti dall'apprendimento che è implicito nel monitoraggio (e su questo punto entra ancora in causa il MM), confluiscono nel piano strategico. Tuttavia la definizione di un piano preliminare e la sua successiva esecuzione sono superate dalla necessità di aggiustamenti continui sia strategici che organizzativi per cui viene meno il rapporto sequenziale piano-esecuzione e l'orizzonte temporale della pianificazione formale è destinato ad accorciarsi.

Il monitoraggio implica un'analisi dell'efficienza e dell'efficacia del MB corrente, la verifica se la creazione di valore all'esterno e all'interno potrà continuare regolarmente o meno. Di conseguenza individua quali aggiustamenti sono necessari dal punto di vista sia strutturale, cioè delle caratteristiche delle componenti, sia del funzionamento. La questione di come le componenti si influenzano nella creazione di valore è pertanto un aspetto di grande rilievo.

Il monitoraggio può portare a confermare sostanzialmente il $\mathrm{MB}$, oppure ad apporre solo limitate variazioni quantitative alla struttura patrimoniale, al conto economico e al livello dei rischi, oppure anche ad ampie ed incisive modificazioni delle componenti. Può indurre alla decisione di innovare il $\mathrm{MB}$ o di avviare processi di migrazione verso altri $\mathrm{MB}$. Ciò può risultare una scelta obbligata quando si stima che il MB corrente stia diventando obsoleto, ormai troppo rischioso e non più attrattivo.

La complessità del monitoraggio dipende dall'assetto del portafoglio delle attività, delle varie linee di business e quindi dal grado di diversificazione della banca. Il MB può risultare molto articolato per cui il monitoraggio richiede analisi articolate (dei singoli business) da ricomporre a sistema unitario per tenere conto delle interdipendenze che rappresentano una fonte di creazione di valore oppure di maggior rischio. Quindi quando si parla di RMB ci si riferisce al rischio del portafoglio complessivo all'interno del quale vi sono i rischi dei singoli business che possono variare anche molto fra di loro.

Ho richiamato il grado di complessità del monitoraggio del MB per sottolineare l'importanza del processo e del ruolo che svolge al riguardo il MM. Intanto dobbiamo ricordare che il RMB dipende da diversi profili del MM che sono anche leve per mitigarlo. Mi riferisco in particolare alla flessibilità al cambiamento, alla capacità di leggere e recepire le dinamiche dell'ambiente, alla capacità di rispondervi in modo rapido e adeguato. Si tratta di aspetti riferibili all'organizzazione della banca e soprattutto al management. 
Questo se da un lato definisce il MB dall'altro ha il compito di governarlo e quindi assume la natura di componente essenziale nel coordinare e influenzare le altre componenti. Pertanto le modalità di funzionamento del MM assumono una valenza determinante nel configurare il RMB.

La rischiosità derivante dalle componenti intangibili del MB deve essere tenuta sotto controllo e mitigata, attraverso un continuo monitoraggio organizzativo per evitare che quando emergono rischi derivanti dalla domanda, dalla concorrenza o da cambiamenti della regolamentazione o dalla innovazione tecnologica la banca sia in grado di reagire con adattamenti o con risposte innovative e non si manifesti un rischio latente di inadeguatezza organizzativa e manageriale ad affrontare il cambiamento esterno. Quindi, riassumendo: le performance del MB sono messe in discussione sia dal dinamismo dei fattori ambientali di riferimento sia dalla capacità delle componenti del MB di adattarsi e di cambiare per rispristinare un nuovo rapporto di coerenza con lo stesso ambiente. Si tratta da un lato di prevedere gli eventi e i cambiamenti esterni che impattano sulle performance: vi è pertanto un problema di valutazione degli scenari; dall'altro lato, occorre che esista la capacità della banca di ridurne l'impatto, anche anticipandoli con cambiamenti opportuni o mitigandoli agendo sempre sui fattori interni. Vi è quindi un problema di efficacia del MM.

Tra i fattori del dinamismo ambientale appaiono rilevanti: l'andamento della domanda, la dinamica dei mercati e la condotta della concorrenza, i cambiamenti della normativa. Le banche si trovano a dove fronteggiare un rischio ambientale di MB al variare di ciascuno questi fattori che possono presentarsi anche contestualmente. Queste minacce, si ripete, possono essere però mitigate ma, dobbiamo aggiungere, addirittura trasformate in opportunità nel momento in cui il cambiamento ambientale dischiude nuove opportunità di business che l'impresa può cogliere.

La mitigazione degli effetti ambientali sulle performance, se dipende dalla flessibilità delle componenti del MB di adattarsi al nuovo contesto ambientale, non può prescindere dall'esistenza di capacità “dinamiche" del MM (Teece 2018). Le capacità dinamiche (così chiamate per distinguerle da quelle "operative" che riguardano la gestione corrente) sono vitali per mantenere la profittabilità dell'impresa perché consistono nella capacità di innovare e di sfruttare le opportunità del futuro; sono quelle richieste per definire il MB come le capacità di intuizione, identificazione, trasformazione, nonché per implementarlo come le capacità di coordinamento, orchestrazione.

Da esse dipendono la rapidità e il grado di efficacia con cui l'impresa adegua le proprie risorse; in altri termini con cui integra, costruisce e riconfigura le competenze interne ed esterne in ambienti in rapida evoluzione. Le capacità dinamiche rappresentano un vantaggio competitivo difendibile perché sono difficili da replicare dai concorrenti. Sono indispensabili per affrontare nuove sfide. Apprendere rapidamente significa creare risorse strategiche: rilevare e modellare opportunità e minacce; cogliere opportunità e mantenere la competitività.

Le capacità dinamiche non sono però sufficienti: occorre considerare anche l'efficacia con cui il monitoraggio si realizza. Non mi riferisco solo alla tempestività delle risposte necessarie ma anche alla capacità previsiva e valutativa degli scenari. La flessibilità necessaria può non essere disponibile al presente ma può essere approntata, con le relative risorse, per tempo se il management se ne rende preventivamente conto. Sono quindi importanti le caratteristiche organizzative del monitoraggio dal processo di analisi e progettazione del MB agli organi che vi intervengono al grado di accentramento/decentramento, ai modelli operativi d supporto.

Nel position paper ho ricordato a titolo esemplificativo due modelli: il modello RCOV (Warnier, Lecocq e Demil 2012) e il modello CANVAS (Osterwalder e Pigneur 2017). Si tratta degli estremi di un'ideale gamma di applicazioni. Il primo modello opera una forte semplificazione delle componenti del MB schematizzando il percorso logico al fine del giudizio di sostenibilità presente e futura del MB, il secondo modello è un vero e proprio manuale pratico con la descrizione dettagliata degli aspetti procedurali e organizzativi. Essi sono utili perché guidano l'analisi e la progettazione del MB ma possono anche funzionare come strumenti di monitoraggio del RMB. La letteratura su questi strumenti propone continui migliorameti e ho già accennato a come sia possibile collegare alle singole componenti del MB un proprio set di "fattori di incertezza" al fine di comprendere l'impatto di tali fattori sulla sostenibilità e profittabilità del MB (Brillinger et al.2020). Ciò vale soprattutto nel caso di innovazione del MB.

Il risk management si trova a svolgere un ruolo critico in questi processi ma il monitoraggio richiede anche contesti organizzativi che agevolino il dialogo e i momenti di confronto, attraverso una serie di meccanismi organizzativi di comunicazione, reporting che favoriscano approcci partecipativi. Non va sottovalutato in questo contesto l'importanza del pensiero visuale cioè delle modalità di rappresentazione/descrizione del MB tramite immagini, grafici, schematizzazioni e altre tecniche che consentono di rendere più tangibili e meno astratti i concetti nei progetti, nei rendiconti sulle performance. Tutto ciò si può far rientrare a pieno titolo tra le pratiche manageriali e quindi tra le componenti dei MM. Si tratta di aspetti organizzativi di grande rilevanza da non sottovalutare mentre spesso si osserva come l'analisi del MB sia confinata nelle relazioni con la Vigilanza e non sia invece una pratica che integra la pianificazione.

\section{Prospettive di ricerca sul rischio di modello di business}

Vi sono evidenze che il management con dirette responsabilità di business è più sensibile all'impatto delle variabili qualitative sulle performance rispetto agli specialisti della funzione di pianificazione strategica e di controllo rischi (Chen et al. 2014). Ciò non giova evidentemente alla gestione del RMB se la funzione di risk management valuta soprattutto i rischi caratteristici. La verifica dell'adeguatezza organizzativa rientra più specificatamente nelle competenze dell'internal auditing e della compliance ma non è detto che tali funzioni di controllo valutino i profili organizzativi dal punto di vista del RMB. Vi è poi da considerare che la funzione organizzazione e personale valuta normalmente singoli aspetti organizzativi e non il funzionamento complessivo dell'organizzazione della banca in un'ottica di tipo sistemico. Valutazioni complessive di coerenza sono però richieste dalla normativa dello SREP al fine della verifica della sostenibilità del MB e della strategia che modifica il MB. 
Si pensi ai report e alla qualità dei flussi informativi nei confronti del Cda della banca che deve approvare il MB e gli indirizzi strategici valutando i rischi che influenzano la stabilità, l'adeguatezza organizzativa e la sostenibilità economica della banca. Il CRO nel presentare al Cda la situazione presente e futura di tutti i rischi della banca, declinandoli per linee di business, si trova pertanto ad affrontare anche il RMB che può rappresentare sulla base di scale di giudizi qualitativi.

E' opportuno quindi che vi sia una stretta collaborazione fra la funzione di pianificazione del MB e della strategia e la funzione di risk management perché nella redazione del piano aziendale si tratta di inserire analisi sia sulle condizioni per "valorizzare i principali driver delle performance" sia sulle "condizioni dannose" per l'evoluzione del MB. Quindi è necessario che a supporto del processo decisionale del Cda la banca disponga delle competenze per effettuare tale analisi e che lo stesso Cda abbia adeguate conoscenze oltre che del MB anche degli strumenti e delle metodologie necessarie per la sua discussione. Si tratta infatti di "competenze essenziali per l'efficace svolgimento del ruolo di amministratore" (Cosma e Nobile 2020).

Devo pertanto richiamare ancora la necessità di sviluppare una maggiore conoscenza sulle relazioni tra le componenti intangibili e quelle tangibili-finanziarie in modo da comprendere come la manovra delle prime influenzi le seconde. Questo tipo di conoscenza è indispensabile per il governo del MB ma anche per una teoria dei MB che ora sostanzialmente manca prevalendo nella letteratura soprattutto la presentazione degli approcci di inquadramento concettuale del MB. Quanto alle conoscenze da sviluppare a livello aziendale si tratta di stabilire se la funzione di risk management debba occuparsene direttamente o in collaborazione con altre funzioni di controllo e con la stessa funzione organizzazione e personale, e se la valutazione del RMB debba rientrare tra le competenze del Comitato rischi, con il supporto delle suddette funzioni, che deve consigliare e supportare il Cda.

Per quanto detto sull'importanza del concetto di MB e delle relative metodologie, credo che si debba convenire sulla necessità che la funzione di risk management rafforzi le competenze e gli strumenti metodologici per valutare e gestire sia il RS sia il RMB e che sviluppi una dialettica più stringente con la funzione di pianificazione e le altre funzioni aziendali. Occorre però che assuma un ruolo di sostanziale indipendenza, di corretta contrapposizione funzionale e di contrappeso nel valutare le ipotesi su cui sono costruiti i MB e i piani strategici evidenziandone, in via preventiva, gli aspetti di criticità e debolezza strutturale e indicando le misure correttive. La sua efficacia viene meno quando le competenze e le skill non sono adeguate perdendo di autorevolezza. Possono così prevalere logiche di mera condivisione e valutazioni che mirano alla validazione. Ciò è spesso frutto anche di una non chiara distinzione dei ruoli tra risk management e pianificazione che indebolisce il $\mathrm{MM}$ e la qualità delle decisioni aziendali (Candolfo 2020). Occorre, si ripete, che a livello aziendale sia sviluppata una funzione di knowledge management a supporto del sistema informativo, di natura non solo quantitativa ma anche qualitativa, su come i fattori intangibili e le loro relazioni si riflettono sulle variabili economiche e finanziarie e sulle performance aziendali.

Si tratta però anche di avviare progetti di ricerca di ampio respiro nel settore bancario basati soprattutto sul metodo dei casi. Nel position paper mostro come il MM sia una componente del MB e che ogni MB richieda un proprio MM. Il RMB emerge quando la coerenza del MM viene meno. E' evidente che tale assunzione debba essere verificata per stabilire se il MM si possa considerare un fattore interno al MB e non un fattore esterno e se, in ogni caso, dovendo risultare funzionale al MB debba essere classificato tra $\mathrm{i}$ fattori firm specific piuttosto che tra quelli firm generic.

Questo contributo suggerisce ipotesi su cui impostare indagini sui MB e sul loro funzionamento. perché la comprensione delle relazioni tra le componenti del MB favorisce il loro efficace monitoraggio. La valutazione degli effetti dei fattori intangibili sulle performance è però tutt'altro che semplice perché implica analisi sul campo basate su casi aziendali (Murthy e Mouritsen, 2011) e non si dispone di una classificazione consolidata di tali fattori la cui criticità varia da business a business (Kristandl e Bontis 2007). Vi è poi da considerare che le relazioni tra i fattori intangibili e tra questi e i fattori tangibili non sono solo di causa ed effetto ma anche di interdipendenza. Si tratta quindi di fare luce su aspetti complessi e dinamici di non facile analisi.

In questo senso segnalo i risultati di alcuni recenti progetti di ricerca (Baravelli e Pesic 2018; Baravelli 2020c) su come stanno evolvendo i MM delle banche e dei gruppi bancari italiani le cui modificazioni sono indotte dall'evoluzione dei MB al variare dei fattori di contesto tra cui la crisi economica, la dinamica competitiva, l'innovazione tecnologica, la regolamentazione, le pressioni all'efficientamento e all'innovazione. Da queste verifiche sono emerse indicazioni di estremo interesse sul RMM. Se i MM non sono flessibili, essi pregiudicano l'evoluzione dei MB. I MM attuali, soprattutto dei grandi gruppi bancari, tendono all'accentramento, sono focalizzati sull'efficienza e presentano i profili di una cultura manageriale in contrasto con le caratteristiche che dovrebbe avere il contesto organizzativo per essere funzionale a promuovere e gestire l'innovazione dei MB. Infatti abbiamo verificato che le migliori performance si riscontrano nelle banche, soprattutto quelle di medie dimensioni, in cui prevalgono sistemi di pianificazione partecipativi e inclusivi e il middle management è coinvolto nella gestione strategica, rispetto alle banche in cui questo è scarsamente coinvolto e svolge ruoli essenzialmente esecutivi.

Ricerche di questo genere vanno promosse in modo più ampio. D'altra parte è la stessa la letteratura sui MB che richiama l'importanza dei MM in diversi aspetti come le competenze e le capacità distintive del management. Ma anche la vigilanza bancaria, nel valutare la sostenibilità dei $\mathrm{MB}$, prevede che siano considerate l'esperienza del management e le sue capacità concretamente dimostrate nell'implementare la strategia. Il nostro approccio è però più ampio. Nel MM vi includiamo elementi sia comportamentali e che riguardano la cultura manageriale sia di struttura che qualificano il MM come team, compreso appunto il ruolo critico del middle management e il modo di lavorare del management che ha ricadute sul personale e sulle relazioni interne. Si tratta di approcci di studio che richiedono di essere modellizzati per poter essere applicati nelle verifiche empiriche a livello sia aziendale che di campioni e di categorie di MB compresi quelli identificati dalle ricerche di tipo finanziario. 
Non è questa la sede per affrontare tale problematica ma può essere utile richiamare alcuni profili del MM a cui ho fatto riferimento nel position paper anche ai fini di classificazione. I MM come i MB si configurano come insiemi di varie componenti di cui si può considerare gli aspetti strutturali sul piano qualitativo e quantitativo. Con riferimento al rapporto tra il numero delle posizioni direttive ai vari livelli - top, middle e operative - sul totale del personale si possono avere modelli manageriali molto gerarchizzati o poco gerarchizzati; gerarchizzati con molto o poco staff, verticalizzati o piatti con una limitata presenza di middle manager; snelli ma con staff articolati. Ma altri rilevanti profili strutturali sono la distribuzione del potere, le relazioni di influenza, le conoscenze, le esperienze e le capacità, la cultura manageriale e aziendale.

Tutti questi fattori presentano fra loro strette relazioni di interdipendenza. I MM si differenziano anche per gli aspetti di comportamento e le pratiche manageriali. Queste ultime sono rilevanti perché influenzano il funzionamento del MM. Riguardano i meccanismi organizzativi che guidano la condotta manageriale, le modalità con cui si svolgono le relazioni interne tra i vari livelli direzionali, più o meno partecipative con cui vengono prese le decisioni, i modelli di analisi e definizione/ridefinizione del MB (si pensi alla BMA), i processi di learning organization e di knowledge management con riferimento soprattutto allo sviluppo di nuova conoscenza per l'innovazione aziendale; i processi formativi del management; le modalità di gestione dei conflitti interni.

I MM nei profili sia di struttura che di funzionamento riflettono la cultura manageriale e più in generale la cultura aziendale. Vi sono MM orientati all'efficienza e MM orientati all'innovazione. Il management è portatore della cultura aziendale che veicola e diffonde nell'impresa; con il suo comportamento; esso stesso è elemento costitutivo dei valori e dei principi di funzionamento dell'impresa. La cultura manageriale è pertanto una rilevante componente del MM. Essa è fondata sui valori, le credenze e le ideologie che condizionano il comportamento del management; la cultura aziendale ne risente ma è influenzata anche da altre componenti soprattutto esterne tra cui anche la regolamentazione.

Il MM riassume gran parte delle componenti intangibili che creano valore dell'impresa e sono quindi un fattore essenziale della capacità di produrre reddito, della vitalità e della competitività dell'impresa. Lo studio dei MM nell'ambito dei MB coinvolge molti filoni della letteratura tra cui la teoria del capitale umano e delle competenze distintive, la teoria della qualità e del miglioramento continuo, la teoria organizzativa e dello sviluppo organizzativo; la teoria manageriale, del people value e della cultura aziendale. Nell'era della conoscenza, in cui stiamo da anni vivendo, la competizione si basa, anche nel settore bancario e finanziario, essenzialmente sulle componenti intellettuali. Mentre le risorse tangibili sono imitabili le risorse intellettuali rappresentano risorse strategiche-chiave non facilmente replicabili.

\section{Considerazioni conclusive}

Nelle riflessioni svolte in queste pagine ho sostanzialmente ripreso i principali aspetti teorici del MB che ho evidenziato nel position paper e che ho commentato nella presentazione fatta nel Webinar del 9 luglio scorso. Qui ho ampliato l'analisi mostrando le ricadute operative del quadro teorico del MB da diversi punti di vista: sul piano delle relazioni tra MB e strategia, sul piano della definizione della nozione di RMB e di RS, sul piano di un possibile ridisegno del processo di pianificazione aziendale. Se si condivide di porre al centro del governo aziendale il MB è necessario considerare le suddette ricadute dando rilievo alla qualità e coerenza del MM.

Con l'introduzione del concetto di MB nel governo della banca si hanno importanti miglioramenti del processo di pianificazione ma anche della cultura aziendale e del MM. L'identificazione del MB, la valutazione della sua sostenibilità messa al centro delle pratiche manageriali, con il supporto delle varie funzioni aziendali e dei responsabili di business, hanno un forte impatto sul processo di apprendimento aumentando la consapevolezza dei problemi e delle decisioni aziendali.

Il framework regolamentare ha indotto le banche in tale direzione ma a livello aziendale occorre approntare una serie di adattamenti e accorgimenti non solo per rispettare i principi indicati dal regolatore ma soprattutto per recepire nel modo migliore anche le raccomandazioni della letteratura al fine di passare dal quadro regolamentare a un quadro organico degli strumenti operativi aziendali. Questo passaggio non è immediato. Esso implica di ovviare ad alcune incompletezze e ambiguità del quadro normativo in modo da integrarlo con la definizione delle metodologie e delle procedure sia per l'analisi del MB sia per valutarne la sostenibilità e il relativo rischio.

Le riflessioni svolte riguardano anche il quadro normativo sulla BMA che non appare sufficientemente chiaro e presenta diverse lacune e ambiguità. Esso non dà una definizione di $\mathrm{MB}$ e di strategia né delle loro relazioni, fornisce solo una nozione di RS ma non di RMB pur ponendo la questione della sostenibilità/rischiosità del MB. La letteratura sui MB, a cui la regolamentazione evidentemente si ispira, consente di dare risposte a queste carenze mostrando come si possa giungere a una definizione di RMB e di RS fra loro compatibili, differenti e complementari. Mettendo il RMB al centro della mappa dei rischi occorre ripensare tale quadro complessivo comprendendovi anche il RMM da collocare tra i rischi organizzativi. E' ciò che abbiamo cercato di fare in queste pagine con la precisazione che si tratta di spunti che devono essere approfonditi dalla ricerca sia a livello aziendale sia a livello di sistema. Ciò pone il problema del ruolo della funzione di risk management, delle competenze necessarie e delle relazioni con le altre funzioni aziendali ma anche della necessità di una più ampia collaborazione tra ricerca interna e ricerca esterna.

Al riguardo, credo che sia necessario ampliare gli approcci valutativi e accettare indicatori di tipo qualitativo. Il regolatore è ancorato ai dati quantitativi ritenuti oggettivi ma in tal modo le valutazioni sono incomplete data la criticità degli intangible che vengono sistematicamente trascurati dalle analisi quantitative. Questa è certamente forse la più importante sfìda culturale perché non è possibile governare il MB senza il monitoraggio delle variabili qualitative tra cui quelle organizzative e manageriali. Occorre quindi che nel MB queste componenti siano rappresentate nel modo più adeguato tenuto conto della loro criticità. 
In questo contributo ho dedicato ampio spazio a illustrare come il MM sia una componente del MB per cui il RMM influenza il RMB e la sostenibilità del MB. Questo approccio non è seguito dalle verifiche sui MB delle banche. Devo al riguardo ricordare che il concetto di MB non è sostanzialmente nuovo per le banche italiane. Dagli anni Novanta del secolo scorso in poi si è sviluppata in Italia un'ampia letteratura sui modelli "strategico-organizzativi" dell'attività bancaria. Questi contributi in realtà parlano antelitteram sostanzialmente di MB. Non solo: la Banca d'Italia a partire dagli anni Novanta ha adottato un modello di vigilanza basato sulla valutazione della coerenza tra ambiente, strategia e assetto organizzativo e il conseguente impatto sulle performance (paradigma ASOP). Occorre quindi prendere atto che vi sono molte analogie tra il modello di vigilanza della Banca d'Italia e la BMA della Bce. Non intendo ritornare su questa questione (Baravelli e Di Antonio 2018), ma solo dire che nella cultura manageriale delle banche italiane, per tutti questi motivi, non dovrebbero essere sostanzialmente estranei il concetto di MB, la sua analisi e il suo ruolo nel governo aziendale.

Maurizio Baravelli

\section{Bibliografia}

- Baravelli M. (2020a), "Modelli di business: definizioni e componenti. Dalla letteratura aziendale e quella bancaria" Business Model SREP: il ruolo del CFO e del CRO, Lavoro congiunto AIFIRM-APB, position paper, n.21.

- Baravelli M. (2020b), "Banche e crisi pandemica: quale impatto sui modelli di business?”, Bancaria, giugno.

- Baravelli M. (2020c), “Come sta cambiando l'organizzazione del lavoro nelle banche”, Rivista Bancaria, luglio-agosto.

- Baravelli M. e Di Antonio M. (2018), "Il nuovo modello di regolamentazione: quali impatti sul settore e sulle strategie delle banche?”, Bancaria, n.7/8.

- Baravelli M e Pesic V. (2018), "Scenari, evoluzione dei modelli di business e cambiamento dei ruoli manageriali nelle banche" Rapporto First Cisl -Sapienza Università di Roma.

- $\quad$ Brillinger A., Els B., Schäfer B., Bender B. (2020), "Business model risk and uncertainty factors: Toward building and maintaining profitable and sustainable business models", Business Horizons 63.

- Brogi M. (2020), "Premessa e sintesi- Covid-19 e governance bancaria, position paper 22”, Risk Management Magazine, maggioagosto.

- Candolfo C. (2020), "Competenze e skill nella definizione e gestione del rischio di modello di business" in Business Model SREP: il ruolo del CFO e del CRO, Lavoro congiunto AIFIRM-APB, position paper, n.2.

- Casadesus-Masanell R. and Ricart J. E. (2010), "From strategy to business models and onto tactics", Long Range Planning, 43(2-3).

- Cosma S. e Nobile L. (2020), "Il ruolo dell'organo con funzione di supervisione strategica", in Business Model SREP: il ruolo del CFO e del CRO, Lavoro congiunto AIFIRM-APB, position paper, n.21.

- $\quad$ Di Antonio M. (2020), "Rischio strategico e di business model”, Business Model SREP: il ruolo del CFO e del CRO, Lavoro congiunto AIFIRM-APB, position paper, n.21.

- Kristandl G. and Bontis N. (2007), "Constructing a definition for intangibles using the resource based view of the firm", Management Decision, 45(9) October.

- Yip G.S. (2004), “Using Strategy to Change Your Business Model”, Business Strategy Review., 15 (2).

- $\quad$ Lorange P. (1980), Corporate planning. An executive viewpoint, Prentice-Hall.

- Murthy V. and Mouritsen J. (2011), "The performance of intellectual capital: Mobilising relationships between intellectual and financial capital in a bank", Accounting Auditing \& Accountability Journal 24(June).

- Osterwalder A. and Pigneur Y. (2010), Business Model Generation: A Handbookfor Visionaries, Game Changers, and Challengers. Wiley, New Jersey (trad. ital.: Creare modelli di business. Un manuale per visionari, innovatori e amanti delle sfide, Edizioni FAG Milano, 2017).

- Osterwalder A., Pigneur Y. and Tucci, C. L. (2005), "Clarifying business models: origins, present, and future of the concept", Communications of the Association for Information Systems, 15(1).

- $\quad$ Seddon, P., and Lewis, G. (2003), "Strategy and business models: What's the difference?" PACIS 2003 Proceedings, 17.

- Warnier V., Lecocq X, et Demil B. (2012), “Le business model, un support à la créativité de l'entrepreneur”, Entreprendre \& Innover, 1 .

- Teece D.J. (2018), “Business models and dynamic capabilities”, Long range Planning, Vol. 51, Issue1, February 2018. 


\section{Pandemic risk: operational aspects}

Bello Camilla, Desando Stefano and Orio Veruska (Intesa Sanpaolo); Giudici Paolo and Tarantino Barbara (University of Pavia).

\section{Abstract}

At the beginning of the COVID-19 pandemic, Intesa Sanpaolo has developed a contagion model aimed at calibrating the measures to be taken to safeguard its employees and the provision of banking services, according to the risk deriving from the external environment.

The model is based on both external and internal views: the combination of such elements provides a holistic picture of the overall contagion risk level, enabling the opportunity to take informed decisions for each geographical area.

One of the most useful external indicators to feed the model is the reproduction index (Rt) provided by the University of Pavia which is calculated using the Poisson autoregressive (PAR) model.

Though more research and development are needed, the proposed model represents a useful tool for supporting the Top Managers in the decision process to continue business as usual safely.

\section{Introduction}

Since the end of February 2020, Intesa Sanpaolo (hereinafter also "ISP") has activated its Emergency Unit to define guidelines aimed at facing the evolution of the COVID-19 pandemic and simultaneously adapting to the requirements of the Prime Minister's Decree of $23^{\text {rd }}$ February 2020.

Jointly with the Cybersecurity and Business Continuity Management and within the Emergency Unit, the Chief Risk Officer provides support to the Top Managers there convened for understanding the phenomenon and its evolution and to foster the assumption of informed decisions (e.g., organizational choices, closure or limitations of branch operations, upgrading of IT systems for smart working and addressing of the related security issues). Indeed, the new way of working exposes to potentially increased risks (i.e., data breaches, internal and external frauds due to remote operating processes and controls, contingency plans of key suppliers and resilience of the supply chain) that must be identified and addressed; above all, it remains essential to continue business as usual safely and to anticipate measures to be taken locally, according to the evolution of the infection, balancing the needs of doing business with security and respect of the Government's measures.

Therefore, Intesa Sanpaolo decided to develop a contagion model aimed at calibrating the measures to be taken to safeguard its employees and the provision of banking services, according to the risk deriving from the external environment.

Based on such experience and on the research by the University of Pavia, we propose a contagion model, described hereinafter. However, the statements in this article are to be intended as exclusive opinions of the authors and do not represent those of Intesa Sanpaolo Group.

\section{Proposed contagion model}

According to our experience, the contagion risk of a Bank depends on the environment (where the Bank is based, where people work, how concentrated are the employees in a certain location, etc., as this affects Bank's employees and Bank's opportunity to carry out business as usual).

Consequently, a contagion model should be developed considering both external and internal perspective and pursuing the following key objectives:

- understand the epidemic dynamic of the COVID-19 virus (so-called external view);

- identify geographical areas where the contagion trend could get out of control and have a significant impact for a Bank (so-called internal view);

- $\quad$ suggest a set of countermeasures to be analyzed to reduce the risk exposure and to protect employees and assets/services provided (e.g., close one or more branches, reduce opening hours).

Such model should envisage several steps (see Figure 1), from the collection of relevant data for populating the risk indicators to the provision of feedbacks useful to support the Top Management in the decision-making process.

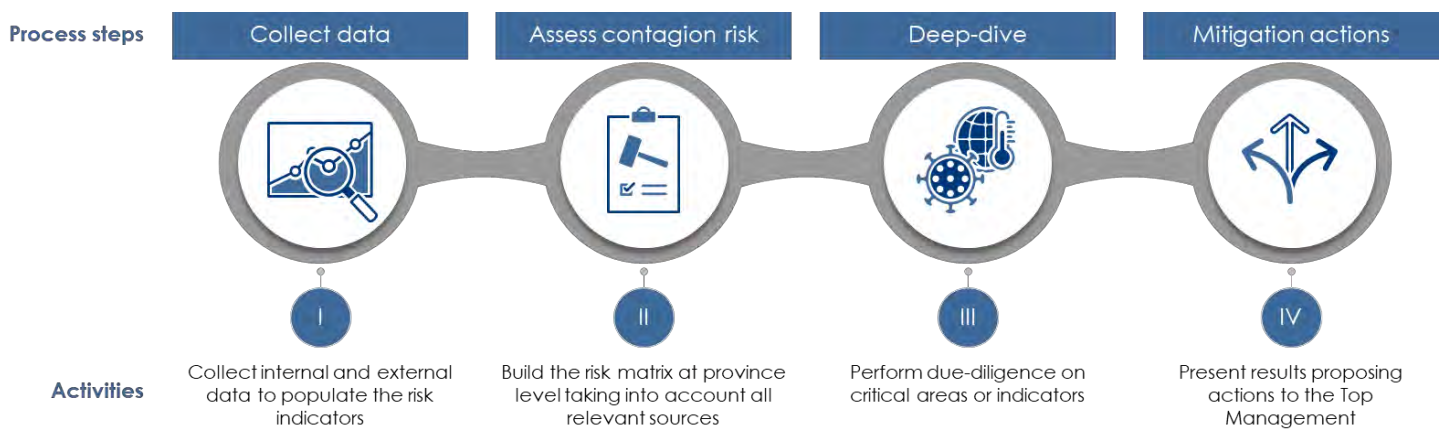

Figure 1: Steps of the contagion model 
Based on our experience, the combination of a limited number of internal and external indicators (see Table 1) can define the risk level of a certain geographical area (Italian region or province).

Consequently, the data collected in the first step should include indicators possibly referred to the specific region or province under assessment:

- the most useful external indicators are the reproduction index (Rt) and those issued by the Italian Ministry of Health, that provide information concerning probability of transmission and severity of the disease;

- the internal indicators should focus on the features of the Banks (e.g., concentration in specific areas or buildings) and the spread of the disease within Bank's employees and premises.

\begin{tabular}{|l|c|c|c|}
\hline \multirow{2}{*}{ Indicators (at region or province level) } & \multicolumn{2}{|c|}{ External } \\
\cline { 2 - 4 } & $\begin{array}{c}\text { Provides input on } \\
\text { Probability of } \\
\text { transmission }\end{array}$ & $\begin{array}{c}\text { Provides input on } \\
\text { Severity of the } \\
\text { disease }\end{array}$ & \begin{tabular}{c} 
Internal \\
\hline Government measures applied (e.g. lockdown)
\end{tabular} \\
\hline Hospital occupancy ratio & $\checkmark$ & $\checkmark$ & \\
\hline Reproduction rate (Rt) & & $\checkmark$ & \\
\hline Percentage of infected employees & & & $\checkmark$ \\
\hline Percentage of closed branches due to COVID infection & & & $\checkmark$ \\
\hline
\end{tabular}

Table 1: Example of indicators used as input to the contagion risk model

Based on external data, the risk level of a certain region can be obtained by combining the probability of transmission and the severity of the disease, through a risk map (see Figure 2); all regions could be clustered within such risk map.

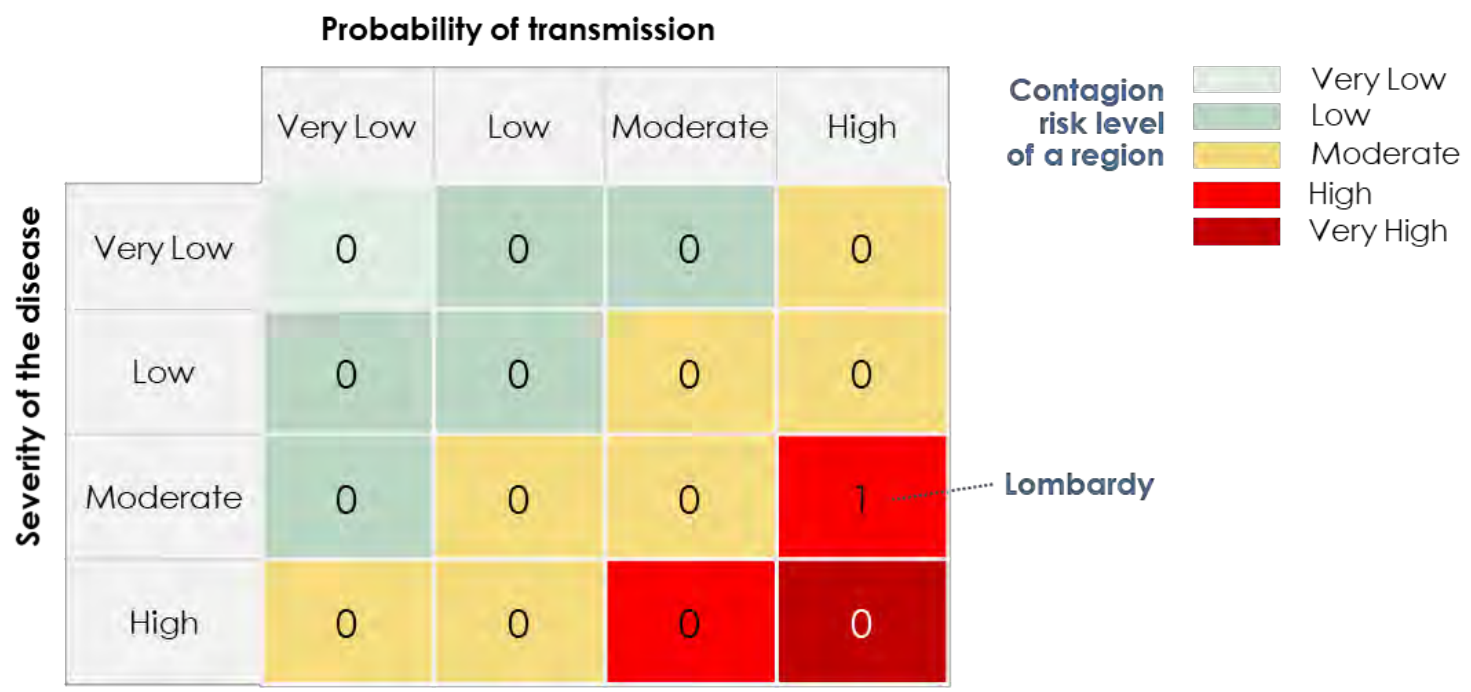

Figure 2: External view: contagion risk level of a region (e.g., Lombardy)

The contagion risk level of the environment affects the Bank's contagion risk level (e.g., employees living or working in a region with high contagion risk could likely contract the virus): therefore, the external view is one dimension of the Bank's risk matrix represented in Figure 4.

The internal view (the other dimension of the Bank's risk matrix in Figure 4) should focus on the assessment of \% and trend of sick employees or of closed branches (i.e., due to COVID infection in a certain area). A useful tool to assess the internal contagion risk level of the Bank in a certain area (region or province) is a decision tree (see Figure 3). 


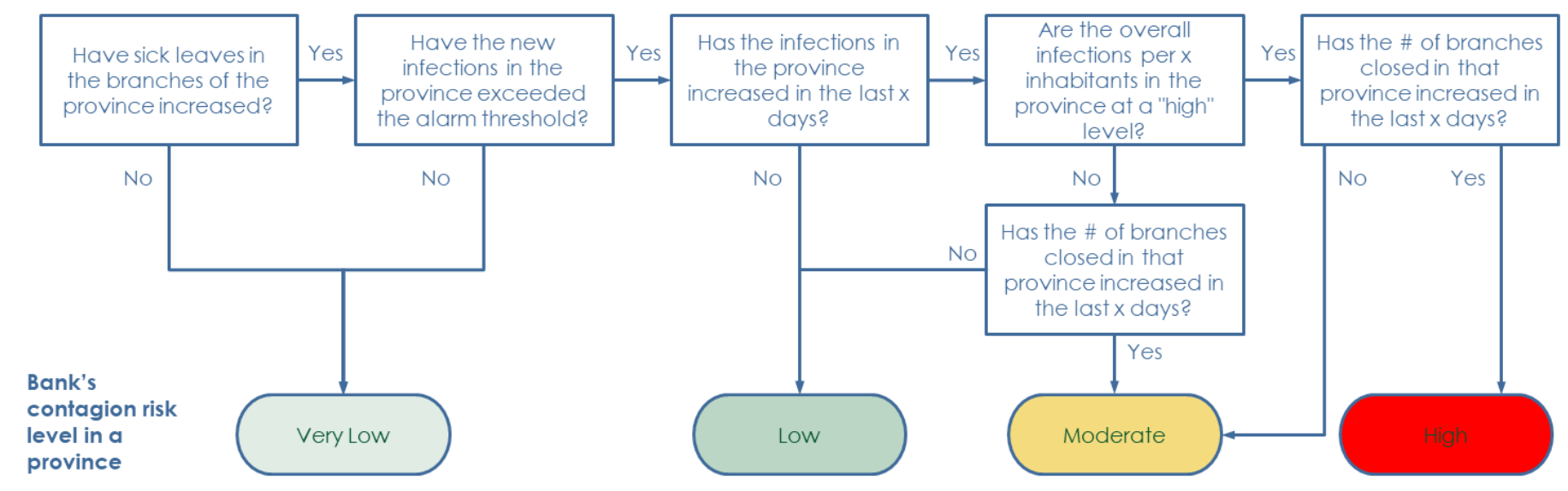

Figure 3: Internal view: decision tree to estimate the internal contagion risk level in a province

The combination of internal and external views in each region/province provides a holistic picture of the overall contagion risk level, enabling the opportunity to classify each province in a specific cluster.

In our example, Lombardy has a High contagion risk level; assuming that, from an internal perspective, Bergamo province has a Low contagion risk level, the overall risk level of that province is Moderate.
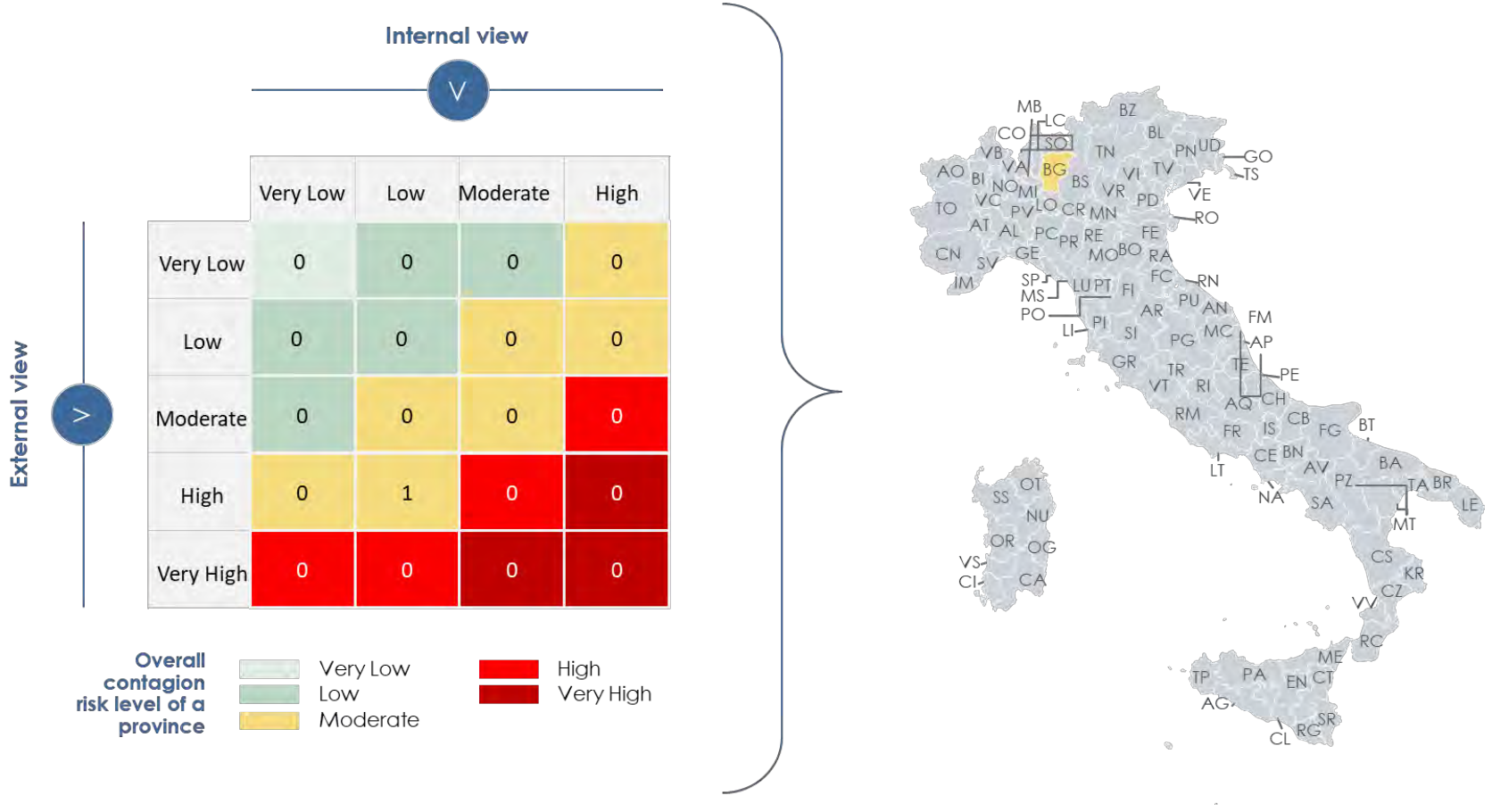

Figure 4: Overall contagion risk level of one province (e.g., Bergamo)

The set of possible mitigation actions to be considered by the Top Management could be associated with the overall contagion risk level of a certain province (see Figure 5 for an example).

\section{Estimation of the reproduction rate Rt}

The reproduction rate of a region could be calculated using the Poisson autoregressive (PAR) model, introduced by Agosto and Giudici (2020), to which we refer for further details.

The PAR model is an extension of the classical Susceptible-Infected-Recovered (SIR) model, in which the logarithm of the daily positives is not assumed to follow a simple one-size-fit-all linear trend but, instead, is assumed to depend on past observed values, thereby allowing to incorporate the effects of contagion shocks into the model.

The PAR model can be easily implemented at the national, regional and/or provincial level. It just requires a time series of daily counts and a software code written in the open source R language, downloadable at the website indicated in the bibliography. 


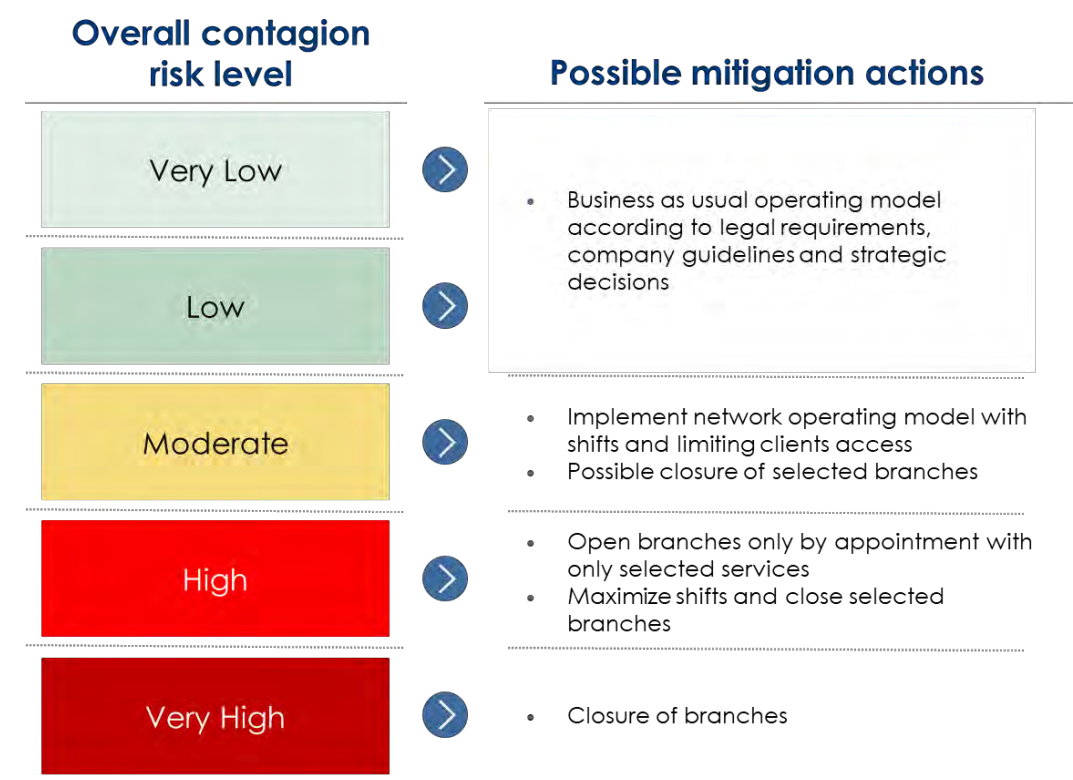

Figure 5: Set of possible mitigation actions according to the risk level

\section{Conclusions}

We believe that a contagion model could represent an effective tool to assess the potential impacts of pandemic risks on Bank's operations and business processes.

Our proposal can be considered as a reliable option where the available data are significant (e.g., larger banks with several branches or several employees in one province).

More research and development are needed, both in the application of the contagion model to financial institutions, and in the improvement of the statistical model, particularly taking into account the effect of decisions such as lockdowns, testing and schools' closures, which should be embedded into explanatory variables.

Indeed, the COVID-19 pandemic has strong implications on risk management; while here we have focused on operational risks, other risks are also heavily affected. For example, Agosto and Giudici (2020b) shows the impact on market risks.

\section{Acknowledgments}

We would like to thank the colleagues of the Cybersecurity and Business Continuity Management who have contributed to the development of the ISP contagion model.

We would also like to extend our appreciation to Boston Consulting Group, involved in the activities underlying the creation and the implementation of ISP's model.

Bello Camilla, Desando Stefano, Orio Veruska, Giudici Paolo and Tarantino Barbara

\section{Essential bibliography}

- Agosto, Arianna and Giudici, Paolo (2020). A Poisson autoregressive model to understand COVID-19 contagion dynamics. Risks, 8(3), 77, MDPI.

- Agosto, Arianna and Giudici, Paolo (2020b). Covid-19 and digital finance. Digital Finance 2, 159-167, Springer.

- R-studio software (2020 edition). https://rstudio.com. 


\section{The resilience of green stocks during COVID-19: a clustering approach}

Giovanni Maria Bonagura (Prometeia), Luca D’Amico (Prometeia), Alessio Iacopino (Prometeia), Lorenzo Prosperi (Prometeia SpA, corresponding author), Lea Zicchino (Prometeia)

Article submitted to double-blind peer review, received on 4th November 2020 and accepted on 28 th November 2020

\section{Abstract}

There is growing evidence that ESG investments have demonstrated higher resiliency to the COVID-19 pandemic shock. While the performance of mutual funds is largely documented, there is limited evidence on stocks, especially in the European market. In this paper we focus on the environmental dimension of firms and we identify a green cluster among listed companies in the EU using a comprehensive database of environmental information. We let the data speak: we identify three clusters of firms (green, non-green and brown) by using clustering techniques and we evaluate their financial performances (return, risk and liquidity) over the full sample period and around the COVID-19 Crisis. We find that green firms yielded a lower return than non-green firms, especially after the Paris Agreement. However, in March 2020, green firms performed better than the other clusters. We find evidence that the COVID-19 period is not a special case, since green firms perform generally better during market contractions. We then extend standard asset pricing models by including the Green Risk Factor, the difference between the green and brown portfolios' returns and we find that the Factor is significant for a large fraction of firms suggesting that climate risk is priced in stocks.

Keywords: ESG, CSR, SRI, COVID-19, Market Crash, Sustainable Investing, Unsupervised Learning, Clustering Methods, KMeans Clustering

JEL: C38, G01, G11, G12, G14, G23, G32, G41, M14

\section{Introduction}

COVID-19 pandemic forced governments to impose social distancing and lockdown measures leading to a collapse in the price of risky assets and spikes in market volatility in anticipation of the fall in economic activity globally. The drop in equity prices was the fastest ever, while the contraction at the through was half the size of that observed during the Subprime Crisis in 20082009 (IMF (2020)). However, there is growing evidence that sustainable and more generally ESG asset classes have been more resilient to the shock. According to Briére (2020), 62\% of large-cap ESG funds outperformed MSCI world index in March. According to Ferriani and Natoli (2020), investors have especially demanded low-ESG risk equity funds and, surprisingly, particularly those with low environmental (instead of social or governance) risk. Compositional effects might explain this result. By construction, ESG funds tend to overweight sectors that have been less exposed to the recent shock, such as health care and tech, and underweight those that have been most impacted, such as transport, energy, materials. However, according to their analysis, other confounding factors, such as exposure to sectors or regions hit hardest by the crisis or market size, do not completely explain larger inflows into ESG funds. Pastor and Vorsatz (2020) analyze actively-managed equity mutual funds in the US market during the COVID-19 crisis and they also find that more sustainable funds (according to Morningstar), and particularly those that are more environmentally sustainable and those that employ exclusion criteria in their investment process, received relatively more net inflows than less sustainable funds within the same style group. These findings are not specific to the COVID-19 crisis; Nofsinger and Varma (2014) showed that socially responsible mutual funds outperformed during other market crises at the cost of underperforming during non-crisis periods. In figure 1 we plot monthly net flows of European equity mutual funds from January 2018 to May 2020 for funds classified as ESG/Green or which claim to have investment strategies based on ESG/Green factors and other funds. Over the sample period, ESG/Green funds flows are more stable than traditional funds even if the former have been only recently introduced. This is relevant especially when markets collapse, as during the last quarter of 2018 and after the outbreak of COVID-19. While traditional funds suffered high outflows in February and hit a low in March 2020 (-112 euro billions), ESG labelled funds were not dramatically impacted; indeed, data do not show massive disinvestments from ESG-labelled funds. Albuquerque et al. (2020) focus on stocks' performance rather than fund flows and find that firms with higher Environmental and Social ratings had significantly higher returns, lower return volatility, and higher operating profit margins during the first quarter of 2020. Other explanations for the better performance of sustainable assets during market crisis point towards market segmentation (investors with different characteristics and strategies can invest separately in the ESG and conventional ETF market segments) or loyalty effects (firms invest in ESG policies to create a more loyal customer base and consequently a less price-elastic demand). Albuquerque et al. (2020) find support for the loyalty theory. In this paper we focus on equity performances of green firms. While mutual fund performance during the COVID-19 crisis is largely documented, there is limited evidence on how stocks, and in particular in the European market, behaved. Moreover, identifying green firms is not an easy task because ESG disclosure by firms might be not fully reliable (i.e. green washing) ${ }^{13}$. Many papers rely on environmental scores produced by different data providers to identify green firms. However, Berg et al. (2020) document the large divergence between ESG ratings across agencies. Some papers identify green firms by taking the best performers according to these scores, i.e. by selecting firms whose score is above certain thresholds. However, the choice of the threshold, like specific percentiles of the score distribution (as in Alessi et al. (2020), Görgen et al. (2019), Albuquerque et al. (2020)) can be subjective and impose an a-priori on the size of the green cluster.

\footnotetext{
${ }^{13}$ see Federal Trade Commission: https://www.ftc.gov/enforcement/rules/rulemaking-regulatory-reform-proceedings/ green-guides
} 


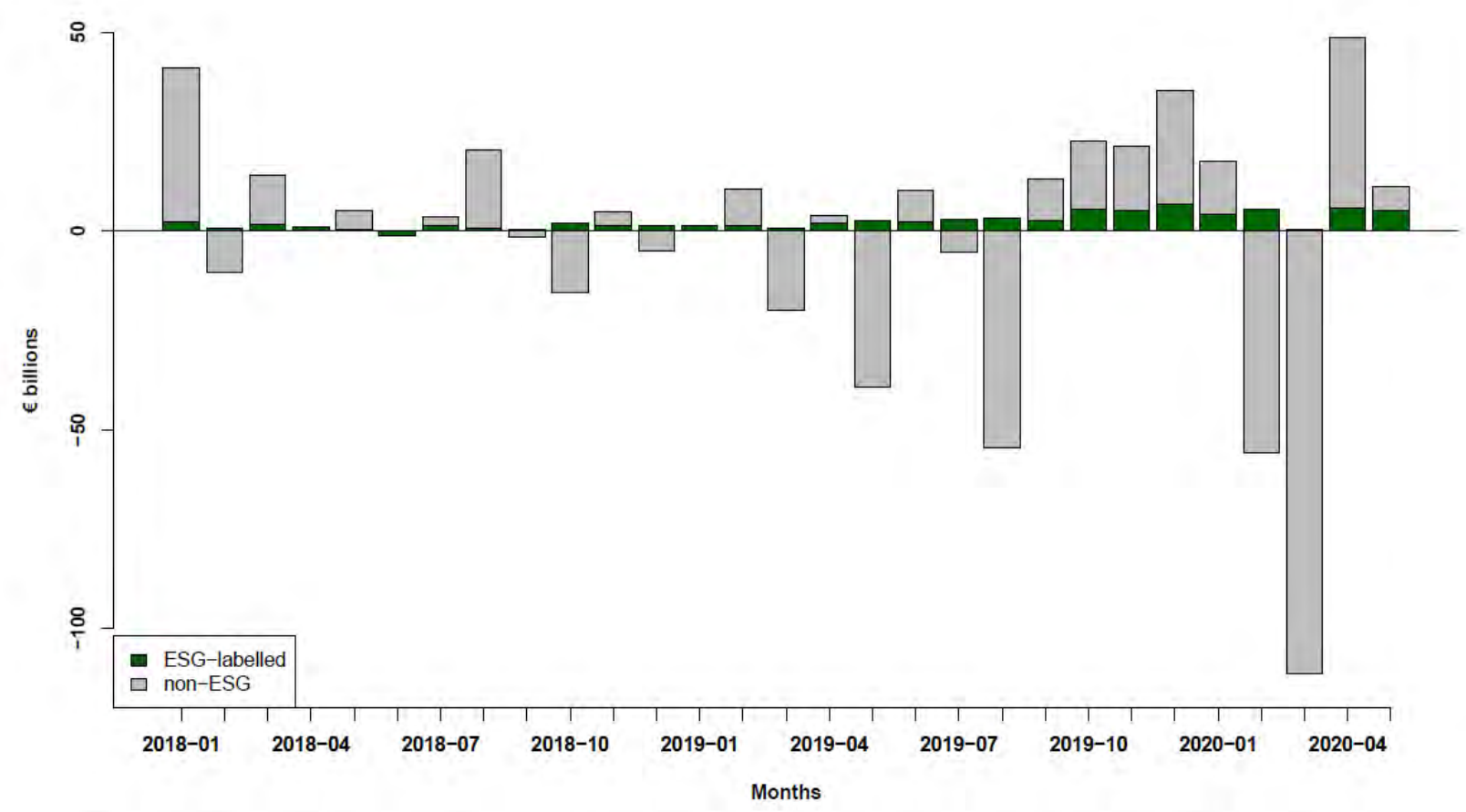

Source: Prometeia analysis and estimates on internal database and external data providers. Data as of May 2020.

In this paper, for each calendar year we identify a green cluster of firms among EU listed companies using a comprehensive database from January 2005 to July 2020 of environmental information at date/firm level from Refinitiv.

For each firm we collected data on emission intensity (Emission/Total Revenue) and four environmental scores (Environmental Pillar Score, Emission Score, Resource Use Score, Environmental Innovation score) constructed by Refinitiv. Differently from previous studies, our objective is to let the data speak by identifying green firms with clustering techniques, instead of creating an aggregate score and selecting the best performer based on this score.

Clustering techniques seek to partition observations in a dataset into distinct groups so that the observations within each group are quite similar to each other, while observations in different groups are different from each other. This approach has several advantages.

First, we do not need to take a stance on the relative importance of different indicators in selecting green firms as the procedure might assign more relevance to some variables to identify specific clusters. Second, the cluster size might change from year to year according to the distribution of the environmental variables among listed firms. We claim therefore that this approach involves less subjective choices in the identification of green firms.

We find quite a large number of green firms. Moreover, green firms are generally bigger than non-green and brown firms (i.e. firms in sectors with large emission levels), maybe because larger firms have more incentive/resources to disclose environmental data. Moreover, the sectorial distribution of green firms is quite heterogeneous and they are not necessarily concentrated in low emitting sectors.

We then evaluate the financial performance of the three clusters (green, non-green and brown) over the full sample period, from January 2006 to July 2020 and around the COVID-19 crisis. When considering stock performance, we find that green firms offered a lower return to stockholders than non-green firms over the full sample period. As suggested by Bolton and Kacperczyk (2020b), investors have only recently become aware of the urgency of climate change.

Consequently, we also evaluate the financial performance splitting the sample in two sub-periods, before and after the Paris Agreement (before and after 2013), and we find the same result.

This is consistent with the view that green firms offer lower compensation because they are a hedge against climate risk. However, green firms outperformed brown firms over the same period. During the market stress induced by the COVID-19 crisis, green firms performed better than both brown and non-green firms, in line with recent literature (Albuquerque et al. (2020)).

We also investigate the performance in terms of risk-adjusted returns in a rolling window setup. On average, we find superior risk-adjusted performances of green firms over the full sample although after 2013 differences among clusters tend to disappear, except during the COVID-19 crisis when green firms outperformed again.

We also proxy the liquidity of the stocks in our database with their bid-ask spread over the mid-price and find that green firms are more liquid than the other two clusters, and the difference in liquidity increases in March 2020.

To investigate whether green firms perform generally better in periods of market stress (Nofsinger and Varma (2014)), as in Alessi et al. (2020) we calculate a Green Risk Factor as the difference between the returns of the green and the brown portfolio. We find that this factor is negatively correlated with the Fama \& French market risk premium and is generally positive during periods of market correction. Moreover, we estimate a standard CAPM and a 3 -factor model using the return on the green, non- 
green and brown portfolio as dependent variable. Consistently we find that the green portfolio has beta below one (i.e. they are defensive stocks), while the other two groups have beta well above one (cyclical stocks).

To get a better understanding of the evidence that climate risk is priced in stocks, we extend the standard 3-factors model of Fama and French (1993) by including the Green risk factor. We find that this factor is significant for around 30\% of the firms at a $10 \%$ significance level in line with the other well-established factors in the literature (HML and SMB).

We also find that green firms have generally positive exposure to the green risk factor while the opposite holds for brown firms. However, there are firms whose exposure to the factor is not aligned with cluster identification, suggesting that markets consider these firms greener (or browner) in comparison with the environmental information that the firms produce.

The rest of the paper is organized as follows. Section 2 describes the dataset. Section 3 is devoted to explaining the methodology: the clustering approach identifying green firms, the measure of financial performance we used in our analysis and the extended asset pricing model. Section 4 describes the results of the clustering procedure and the relative performance of the green cluster of firms over the full sample period, during the COVID-19 crisis and pre/post Paris Agreement periods. Section 5 concludes.

\section{Data}

Our sample consists of companies listed on the STOXX Europe Total Market Index in July 2020, with the exclusion of financial and real estate companies, i.e. companies with NACE-1 digit level equal to K or L.

In order to identify green firms, we collect ESG data from Refinitiv; in particular, we focus on an emission intensity measure and various environmental scores: Sales/Emission ratio, Environmental Pillar Score, Emission Score, Resource Use Score, Environmental Innovation score ${ }^{14}$.

The inverse of the emission intensity (i.e. Sales/Emission ratio) has been included for two reasons. Firstly, it is an indicator widely used in the literature to identify best performers in terms of emission policy. Secondly, unlike the Refinitiv scores that are industry-specific and measure the relative performance of a firm within its own sector, emission intensity is an absolute measure. ${ }^{15}$

Furthermore, in figure 2 we present the Indicators' correlation Heatmap; it is worth noticing that the sales/emissions indicator is not necessarily correlated with the other environmental indicators and this is true over the years. In table 7 we provide summary statistics for each Environmental indicator, mentioned above.

Figure 2: Environmental Indicators' correlation HeatMap

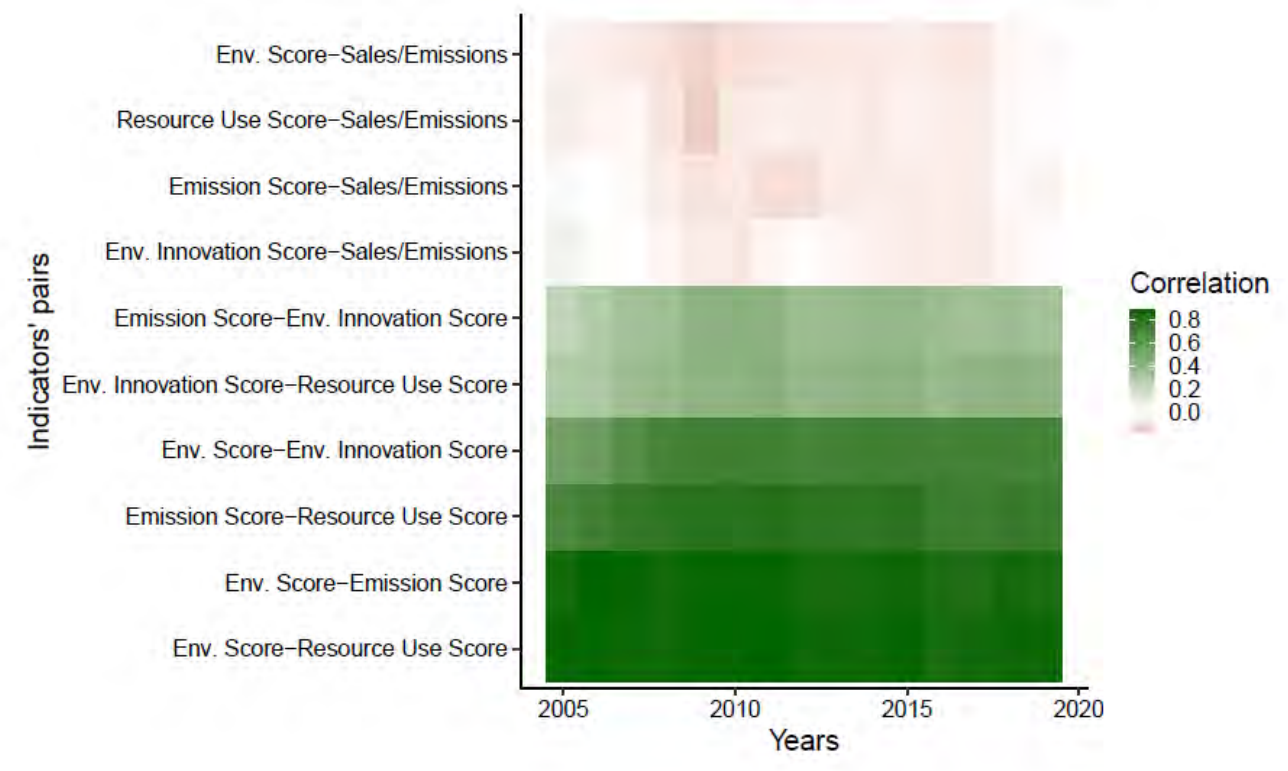

In this paper brown firms are not identified using our clustering procedure, but as firms that belong to sectors that are more exposed to climate risk, independently from their relative ranking based on the environmental data considered.

Following Alessi et al. (2020), the first step of our analysis consists in selecting, for each year, only the securities that do not belong to the sectors defined as CPRS (Climate policy-relevant sectors) according to their associated NACE-2 digit level.

Based on Eurostat data, in table 1 we identify the CPRS sectors as those which, in descending order by level of GHG emissions, contributed to $85 \%$ of total GHG emissions between 2008 and 2018; the firms which operate in CPRS sectors are labelled as "Brown".

\footnotetext{
${ }^{14}$ See the Appendix A for a detailed description of the scores.

${ }^{15}$ Note that, as explained in the Refinitiv's ESG methodology guide, in order to calculate the environmental category scores, the industry group is used as the benchmark. In addition, some indicators are industry-specific, thus they are not relevant for some sectors; in this case they are excluded from the calculation.
} 
Table 1: European Union Climate Policy Relevant Sectors (CPRS) with their relative contribution to total GHG emission, based on Eurostat data

\begin{tabular}{|c|c|c|}
\hline NACE-2 digit lvl. & $\%$ of total GHG emissions (2008-2018) & Cumulative \% \\
\hline D35 - Electricity, gas, steam and air conditioning supply & 31.5 & 31.5 \\
\hline A01 - Crop and animal production, hunting and related service activities & 13.4 & 44.9 \\
\hline H49 - Land transport and transport via pipelines & 5.5 & 50.3 \\
\hline C23 - Manufacture of other non-metallic mineral products & 5.5 & 55.8 \\
\hline C24 - Manufacture of basic metals & 4.9 & 60.7 \\
\hline E37-E39 - Sewerage, waste management, remediation activities & 4.6 & 65.3 \\
\hline C20 - Manufacture of chemicals and chemical products & 4.3 & 69.6 \\
\hline H51 - Air transport & 4.0 & 73.6 \\
\hline C19 - Manufacture of coke and refined petroleum products & 4.0 & 77.6 \\
\hline H50 - Water transport & 3.3 & 80.9 \\
\hline B05-B09 - Mining and quarrying & 2.3 & 83.3 \\
\hline F41-F43 - Construction & 1.8 & 85.0 \\
\hline
\end{tabular}

In other words, we exclude the possibility that Brown firms are identified as green. While this hypothesis might look very subjective, given that some brown firms are largely investing to diversify their core business and in adaption technologies, it is quite reasonable to assume that these sectors are highly affected by transition risk (i.e. carbon pricing policies). In identifying green firms, a crucial aspect is how to treat missing data. Indeed, a large fraction of firms does not report any environmental information, especially at the beginning of the sample period. Our approach is to penalize non-reporting firms compared to reporting firms. Then, when a firm presents missing data for an indicator, we replace it with the minimum value observed for the indicator in each specific year minus an arbitrary value. Moreover, due to the presence of outliers and indicators with different scales, we standardize the data calculating the rank of each Refinitiv indicator every year, effectively minimizing distortions in the clustering procedure ${ }^{16}$. One of the main contributions of this study is the clustering technique for the identification of green firms, which is described in the next section.

\section{Methodology}

\section{Clustering Methodology}

As mentioned in the introduction, to avoid bias in the choice of the green firms we adopt a data-driven approach. In this work, we propose an innovative approach that identifies a time-varying number of green firms using unsupervised learning clustering techniques. In particular, we apply the K-means algorithm with squared Euclidean distance as a dissimilarity measure in order to find homogenous groups of firms, according to the environmental indicators mentioned before. Therefore, we want to solve this optimization problem

$$
\min _{C_{1, t \ldots C_{1, t}}}\left(\sum_{k=1}^{K} W\left(C_{k, t}\right)\right)
$$

with

$$
W\left(C_{k, t}\right)=\frac{1}{\left|C_{k, t}\right|} \sum_{i, i^{\prime} \in C_{k, t}} \sum_{j=1}^{p}\left(x_{i, j, t}-x_{i^{\prime}, j, t}\right)^{2}
$$

where $x_{i, j, t}$ is firm $i$ rank on feature $j$ at time $t . W\left(C_{k, t}\right)$ is a measure of dissimilarity between the observations within the $k$ th cluster (i.e. within-cluster variation), defined as the summation of all of the $k$ th pairwise squared Euclidean distances between the observations, divided by the $k$ th total number of observations. The K-means algorithm allows us to solve this problem such that the total within-cluster variation $\sum_{k=1}^{K} W\left(C_{k, t}\right)$ is minimized.

This type of algorithm requires the number of clusters that we expect in the data as input; given that the main aim of this procedure is to avoid arbitrary choices, the optimal number of clusters is estimated from the data. The identification problem of the optimal number of clusters is not an easy task: as a matter of fact, a vast number of tests have been introduced in the literature to solve this problem but there is no consensus about which is the most appropriate to use. For this reason, we decide not to rely on a specific test but instead consider the results of different tests, as implemented in Malika et al. (2014). Finally, to aggregate the results of different tests we take the median reported number of clusters in each year. As a result, we find an optimal number of clusters, constant over time, equal to three. In order to assign different labels to the clusters obtained for each year, we assume that the one with the highest average of the medians of each ranked Refinitiv indicator should be labelled "green" and the other two clusters form a unique one called "not green". This classification allows us to build three different market-value weighted portfolios (green - not green- brown) for each month, based on the previous year classification. This is because, as environmental information is collected and disclosed yearly, we assume that during any given year an investor can only consider the previous year information. We then define the "Green Risk Factor" as the difference between the monthly returns on the green and the brown portfolio.

${ }^{16}$ Since data are standardized using the rank function, the choice of arbitrary value does not affect the clustering results. 
For each portfolio, we calculate monthly US dollars returns from January 2006 to June 2020, a risk adjusted score and the bidask spread over the mid-price

$$
\operatorname{BDSKP} M_{i, t}=100 \times \frac{P_{i, t}^{A}-P_{i, t}^{B}}{\frac{P_{i, t}^{A}+P_{i, t}^{B}}{2}}
$$

Then we evaluate the performance of the portfolios, based on these indicators, considering two different time spans, the fullsample period and the COVID-19 months.

The risk-adjusted score, which brings together various risk-adjusted measures in a single indicator, allows an immediate comparison between portfolios by assessing the overall risk-return profile more completely. For each listed company we consider risk and return indicators calculated on a rolling window of 260 days. Risk indicators include the Sharpe Ratio (Sharpe (1994)), Information Ratio (Goodwin (1998)), Appraisal Ratio (Treynor and Black (1973)), K3 (Kaplan and Knowles (2004)), Sortino Ratio (Sortino and Price (1994)) and Treynor Ratio (Treynor (1965)). For each firm, we determine an aggregate score by averaging the rank of the firm according to each indicator. The score values range from 0 (minimum performance) to 100 (maximum performance). Finally, for each portfolio we calculate the overall score as the market-value weighted average.

\section{Asset pricing with the Green Risk Factor}

We then study the role of climate risk in equity prices by extending the standard asset pricing model by including the Green risk factor as in Alessi et al. (2020) and Görgen et al. (2019). In particular, for each firm we estimate the following regression

$$
R_{i, t}=\alpha_{i}+\beta_{i}^{M} M R P_{t}+\beta_{i}^{H} H M L_{t}+\beta_{i}^{S} S M B_{t}+\beta_{i}^{G} G R F_{t}+\epsilon_{i, t}
$$

where $R_{i, t}$ represents the monthly excess return of firm $i$, while $M R P_{t}, S M B_{t}$ and $H M L_{t}$ are respectively the market risk premium and the two factors introduced ${ }^{17}$ by Fama and French (1993), while $G R F_{t}$ is the green risk factor.

The panel data of firms' returns is unbalanced and some firms have a relatively short history of data that might produce inaccurate results. For this reason, when computing summary statistics of the exposure across groups of firms we underweight firms whose estimation results might be less reliable. We follow the approach proposed in Gagliardini et al. (2016), where the weights are used in the second step of the procedure for risk premia estimation. In short, giving weights increase the statistical precision of the estimation of the beta and overcomes the issue of the small sample size relative to the full sample.

\section{Results}

\section{Clustering Results}

Figure 8 shows the boxplot of the indicators' rank divided into groups. The first thing to notice is that our methodology produces three clusters with different characteristics. In the initial period green firms have higher scores in all indicators than non-green firms, while firms with missing values (non-transparent firms) are cat the bottom of the graph. However, over the rest of the period, the positive correlation between green firms and the sales-to-emission ratio disappears: we find more homogeneity in emission intensity between green and non-green firms. Indeed, starting from 2017 non-green firms show a higher value in the sales-to-emission ratio, while all the other indicators are higher for green firms. Figure 9 shows the sectorial distribution of green, non-green (included non-transparent) and brown portfolios. In the graph we show the most relevant sectors in terms of market value, ordered counter clockwise according to their emission level (the sector in the north part of the radar corresponds to the highest emitting sector). We find that green firms do not necessarily belong to sectors with relatively lower emissions. In figure $3 \mathrm{a}$ we present the evolution of the number of firms included in each cluster. First, it can be noticed that the number of green firms increases over time, along with increasing environmental disclosure by firms. Second, the green and brown portfolios have, on average in the sample considered, a similar number of constituents. It should be noted that despite our procedure does not allow for an a-priori classification and therefore there is no guarantee about the stability of the number of firms in each cluster, our green cluster is stable over time, presenting an average turnover of around $88 \%$. As already mentioned, a widely adopted approach in the literature is to identify green firms by selecting those above a certain threshold. The choice of the threshold, like specific percentiles of the distribution, can be subjective, and impose an a-priori on the size of the green cluster. Our approach shows that it is difficult to identify ex ante a unique suitable threshold, since the number of green companies in our sample varies over time. In particular, the share of green firms spans from $9.6 \%$ to $24.2 \%$ in the sample considered. In figure $3 \mathrm{~b}$ we show the evolution of the average market value in each cluster over the average market value among all firms. Values above (below) one for a specific cluster/year tell that on average firms in this cluster are bigger (smaller) compared to the entire market in the year considered. It is clear that green firms are generally bigger than non-green and brown firms. This could be also explained by the fact that larger firms are better at disclosing a large amount of non-financial information and therefore receive higher scores.

\footnotetext{
${ }^{17}$ The three factors for the European market in US dollars together with the risk free rate are taken from Fama\&French website
} 
(a) Number of firms included in each cluster

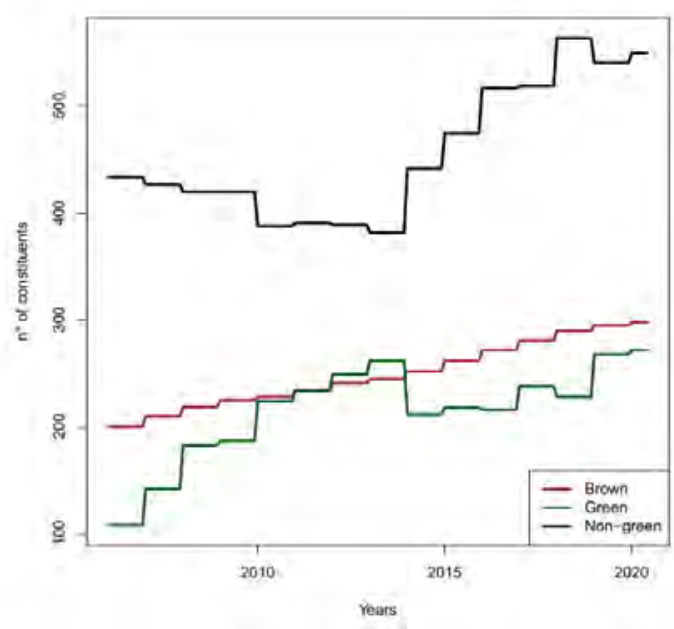

(b) Average market value in each cluster over the average market value among all firms

\section{Performance Results}

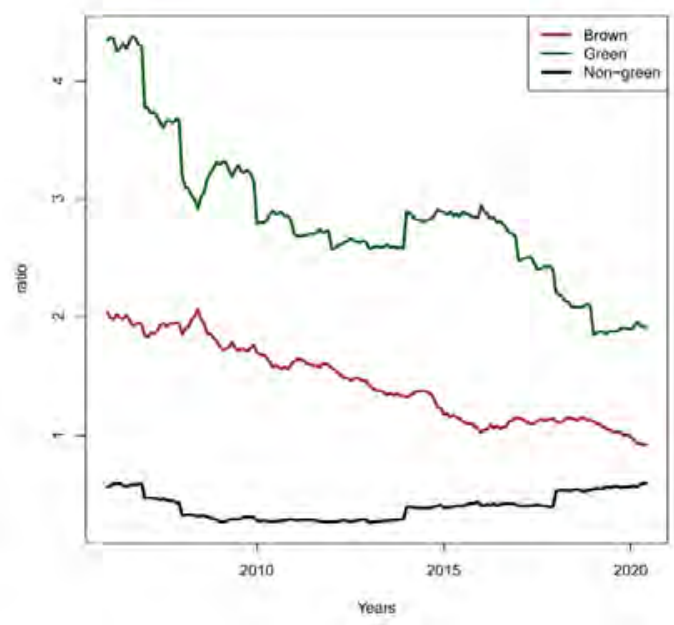

In figure 4a we present the monthly cumulative returns of the green, non-green and brown portfolio starting from 2006 . The three portfolios diverge from 2010, with the brown portfolio that under-performs the other two. Moreover, the green portfolio underperforms the non-green portfolio from 2013, in anticipation of the Paris Agreement, in which occurred in 2015. The underperformance of green firms with respect to non-green firms is in line with the main findings in the literature (Alessi et al. (2020), Bolton and Kacperczyk (2020a), Bolton and Kacperczyk (2020b), Görgen et al. (2019)).

The intuition behind this finding is that investors are already demanding compensation for their exposure to climate risk. However, this interpretation appears in contrast with the finding that green firms outperform CPRS firms, that are supposed to be more exposed to climate risk. According to Görgen et al. (2019), if firms surprise markets by performing relatively worse on environmental issues, by becoming browner, investors discount these firms. Pastor et al. (2019) model ESG preferences and their impact on asset prices.

Figure 4

(a) Monthly cumulative returns of the green, non green and brown portfolios

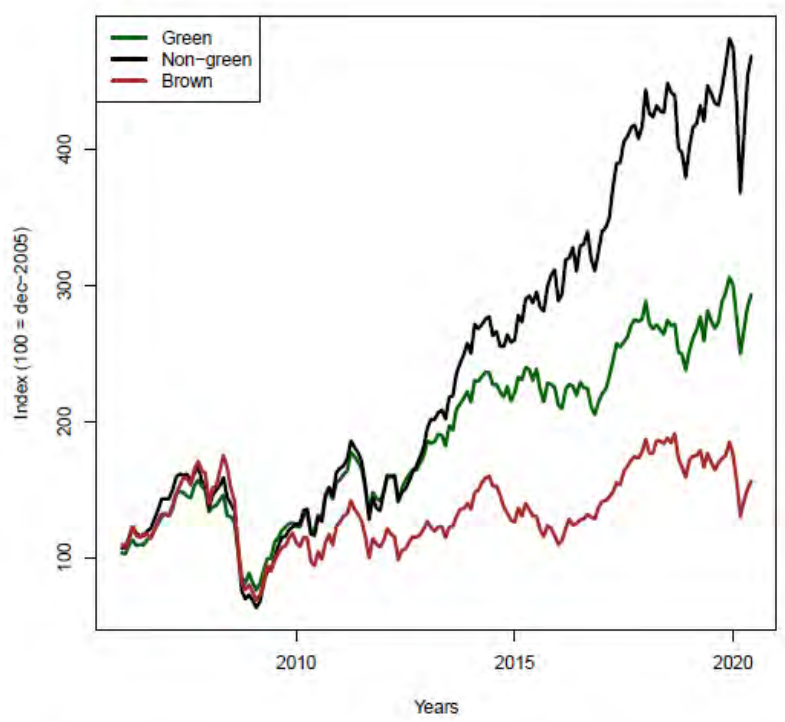

Note: The brown portfolio is composed by firms belonging to Climate Policy Relevant Sectors in July 2020 and each company is weighted in the index according to market size. The green portfolio composition changes every year according to the selection of firms from clustering procedure and according to their market capitalization. (b) Green Risk Factor and Market Risk premium

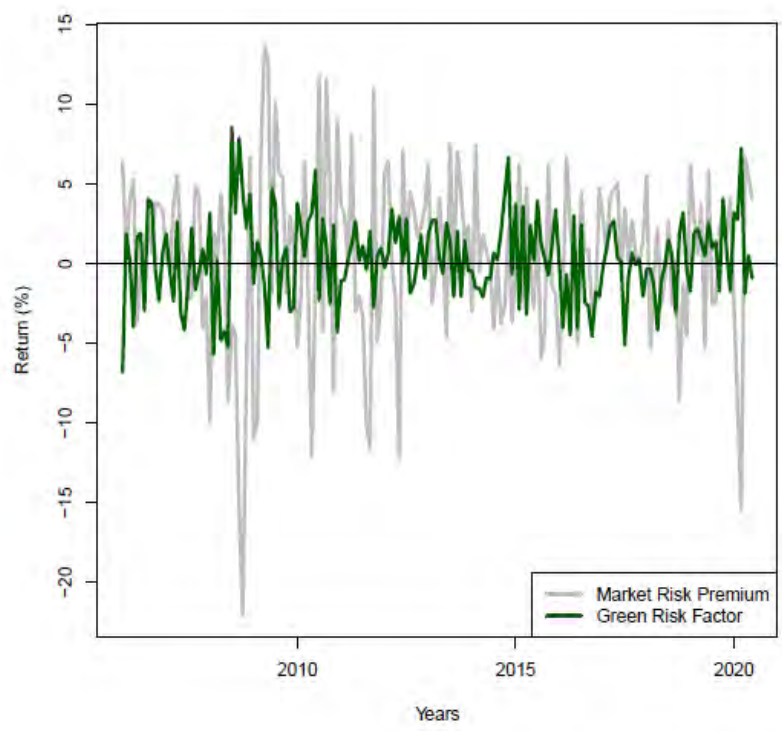

Note: The "Green risk factor" is the difference between the green and the brown portfolio return, while Market Risk Premium is taken from Fama\&French website. 
Investors vary in their ESG preference and invest in a long short green-brown portfolio. In their model the greener the asset, the lower the returns. They also introduce an ESG factor that represents a shift in investor demand, and they show that positive realizations increase green-asset prices even though brown assets earn higher expected returns. Our result is consistent with this view, with increasing environmental awareness green firms are those that performed relatively better than brown firms that are "stuck" in more climate intensive sectors. Moreover, according to our data, green firms are becoming relatively greener over time since the average rank of the indicators increased in the full sample. In table 2 we present summary statistics of returns over the full sample period and pre/post Paris agreement. The over-performance of non-green versus both green and brown portfolios is especially evident in the post Paris agreement period.

To investigate the performance of green firms compared to brown firms during COVID-19 Crisis and before then we calculate a "Green risk factor" as in Alessi et al. (2020) as the difference between the green and brown portfolios (figure 4b). Our result is that in March 2020 this factor is positive (7\%), implying green firms performed better than brown firms.

Table 2: Summary Statistics of returns in the full sample and pre/post Paris agreement

\begin{tabular}{l|ccc|ccc|ccc}
\hline \hline \multirow{2}{*}{ Portfolio Excess Return } & \multicolumn{3}{|c|}{ Pre-2013 } & \multicolumn{3}{c|}{ Post-2013 } & \multicolumn{3}{c}{$2006-2020$} \\
\cline { 2 - 10 } & Mean & Std. Dev. & t-stat & Mean & Std. Dev. & t-stat & Mean & Std. Dev. & t-stat \\
\hline Green & 10.29 & 28.62 & 0.95 & 8.87 & 16.74 & 1.40 & 9.58 & 22.54 & 1.59 \\
Brown & 6.34 & 33.95 & 0.49 & 7.10 & 15.18 & 1.24 & 6.72 & 25.27 & 1.00 \\
Non-green & 12.08 & 41.10 & 0.78 & 14.88 & 15.96 & 2.47 & 13.48 & 29.99 & 1.68 \\
\hline \hline
\end{tabular}

Actually, green firms performed better than the market as well in the same period $(6 \%)$. Moreover, we check whether green firms perform generally better in period of market stress (Nofsinger and Varma (2014)) and find that the green risk factor is on average positive (4.5\%). Finally, the factor is negatively correlated with the Fama \& French Market risk premium (-0.36). When considering risk adjusted performance we find that the green portfolio yields a better risk-adjusted average score than the non-green and brown portfolios especially before 2013. As we show in figure 5a the gap is clear from 2005 to 2013 , and in particular in 2009 and 2011, when Europe experienced the effects of the sub-prime and sovereign debt crises, and then it becomes a bit fuzzier. The gap increases in 2015, when the score of the brown portfolio drops because of the 2014-16 oil glut. Then, the performance of the brown portfolios starts to improve until the COVID-19 crisis, which is characterized by the better performance of the green firms.

Figure 5
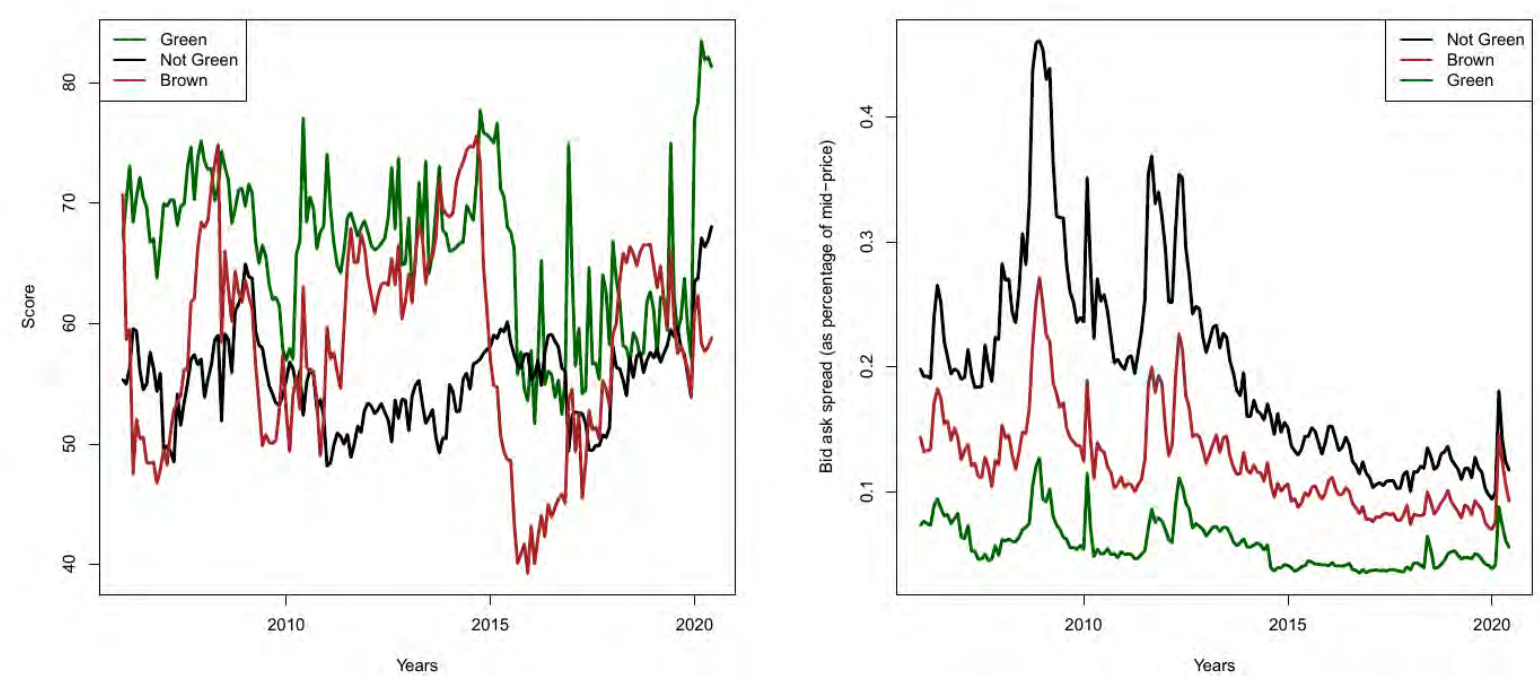

Furthermore, we verify whether green firms are more liquid than brown firms. To this end, we use the bid ask spread over the mid-price (BDSKPM) as our liquidity measure (see Sarr and Lybek (2002)). Figure 5b indicates that there is a clear gap between our three clusters, indeed the bid-ask spread of green firms is lower than that of brown and non-green firms throughout the whole sample period. In line with previous results, we find that BDSKPM of all the three clusters increased in crisis periods (i.e. the subprime crisis, the sovereign debt crisis and the COVID-19 crisis). However, the magnitude of the increase of the $B D S K P M$ of green firms was lower than the other two clusters. In particular during the COVID-19 crisis, the BDSKPM of brown and nongreen firms jumped from, respectively, 0.76 and 0.9 in February 2020, to 0.15 and 0.18 in March 2020; meanwhile for the green firms it increased from 0.04 in February 2020 to 0.08 in March 2020. In other words, during the COVID-19 crisis the liquidity gap between green firms and the others has further increased. This evidence supports the hypothesis that the demand for green securities increased during the crisis relatively to the other clusters.

Our preliminary results suggest that the green portfolio is more resilient than the non-green and brown portfolios. In tables 5-6 we show the betas of the excess return of those portfolios, estimated using a standard CAPM and a 3-factor model. First, we 
find a statistical significance for the market risk premium for all portfolios in both models. We also find that the green portfolio has a beta lower than one, with respect to the market (defensive stocks), and lower than non-green and brown portfolios, which have beta well above one (cyclical stocks). This implies that green firms have lower exposure to systematic risk than brown and non-green firms, leading to better performance during market collapses.

\section{Asset pricing with Green Risk Factor}

In Figure 6 we show the histogram with the frequency distribution of $\beta_{i}{ }^{G}$, the exposure to the Green Risk Factor (GRF). As shown in table 5, we find that the Green Risk Factor (GRF) is highly significant. More specifically, it is significant for $25 \%$ $(32 \%)$ of the firms in the sample, at a 5\% (10\%) significance level, not far from the other well-established factors in the literature (HML and SMB). Firms with positive (negative) $\beta^{G}$ should have larger (smaller) returns when the GRF increases, meaning that they benefit (lose) when climate risk deteriorates. According to our estimates exposures to GRF are quite heterogeneous.

Moreover, the results are line with the sectorial classification. In Figure 7 we represent the sectorial (NACE2 level) weighted average of the exposure to the green risk factor across firms. In particular in the left panel we represent the top 14 sectors according to the weighted average beta and on the right panel the bottom 14 sectors. We find that high emitting sectors exhibit negative Beta's, while low emitters have generally positive Betas. Confirmation of this is shown in Table 6, which represents clustered Betas: the weighted mean is positive for the stable-green cluster ${ }^{18}$, while it is around zero and negative for the nongreen and brown clusters, respectively. This means that on average stable green firms are benefiting from increasing climate risk, measured by the GRF, while the opposite holds for brown firms. However not all green firms have positive beta (around $76 \%$ in the stable-green cluster), and there are brown firms that have positive exposure (approximately $35 \%$ ). This might suggest that markets identify potential environmental weakness/strengths of firms that are not correctly measured by the environmental data and KPI considered in this analysis.

Table 3: CAPM estimates on different portfolio excess returns

\begin{tabular}{lccc}
\hline \hline & \multicolumn{3}{c}{ Dependent variable: } \\
& Green & Brown & Non-green \\
\hline MRP & $0.905^{* * *}$ & $1.086^{* * *}$ & $1.065^{* * *}$ \\
& $(0.020)$ & $(0.029)$ & $(0.027)$ \\
& & & \\
Constant & $0.380^{* * *}$ & 0.013 & $0.646^{* * *}$ \\
& $(0.107)$ & $(0.159)$ & $(0.145)$ \\
\hline Observations & 174 & 174 & 174 \\
$\mathrm{R}^{2}$ & 0.925 & 0.890 & 0.904 \\
Adjusted R ${ }^{2}$ & 0.925 & 0.890 & 0.903 \\
Residual Std. Error $(\mathrm{df}=172)$ & 1.412 & 2.088 & 1.907 \\
F Statistic $(\mathrm{df}=1 ; 172)$ & $2,122.159^{* * *}$ & $1,397.150^{* * *}$ & $1,611.612^{* * *}$ \\
\hline \hline \multirow{2}{*}{ Note: } & \multicolumn{3}{c}{${ }^{*} \mathrm{p}<0.1 ;{ }^{* *} \mathrm{p}<0.05 ;{ }^{* * *} \mathrm{p}<0.01$}
\end{tabular}

Table 4: Fama-French three-factor model estimates on different portfolio excess returns

\begin{tabular}{lccc}
\hline \hline & \multicolumn{3}{c}{ Dependent variable: } \\
& Green & Brown & Non-green \\
\hline MRP & $0.984^{* * *}$ & $1.095^{* * *}$ & $1.123^{* * *}$ \\
& $(0.019)$ & $(0.033)$ & $(0.023)$ \\
& & & \\
SMB & $-0.116^{* *}$ & $0.161^{*}$ & $0.592^{* * *}$ \\
& $(0.049)$ & $(0.085)$ & $(0.057)$ \\
& & & \\
HML & $-0.355^{* * *}$ & -0.052 & $-0.297^{* * *}$ \\
& $(0.044)$ & $(0.076)$ & $(0.051)$ \\
Constant & & & \\
& $0.272^{* * *}$ & -0.019 & $0.496^{* * *}$ \\
Observations & $(0.092)$ & $(0.160)$ & $(0.108)$ \\
$\mathrm{R}^{2}$ & 174 & 174 & 174 \\
Adjusted $\mathrm{R}^{2}$ & 0.947 & 0.893 & 0.949 \\
Residual Std. Error $(\mathrm{df}=170)$ & 0.946 & 0.891 & 0.948 \\
F Statistic $(\mathrm{df}=3 ; 170)$ & 1.196 & 2.075 & 1.401 \\
\hline \hline Note: & \multicolumn{3}{c}{${ }^{*} \mathrm{p}<0.1 ;{ }^{* *} \mathrm{p}<0.05 ;{ }^{* * *} \mathrm{p}<0.01$}
\end{tabular}

\footnotetext{
${ }^{18}$ We define as "stable-green" the firms that, considering only the time span where they were effectively listed, are labelled green in more than $50 \%$ of the
} years. 
Table 5: Percentage of companies that present significant risk factors for different levels of significance

\begin{tabular}{lrr}
\hline & alpha $=5 \%$ & alpha $=10 \%$ \\
\hline const & 4.8 & 13.8 \\
MRP & 99.6 & 99.7 \\
SMB & 53.9 & 60.6 \\
HML & 43.4 & 50.9 \\
GRF & 25.4 & 32.4 \\
\hline
\end{tabular}

Figure 6: Frequency distribution of the Beta green risk factor

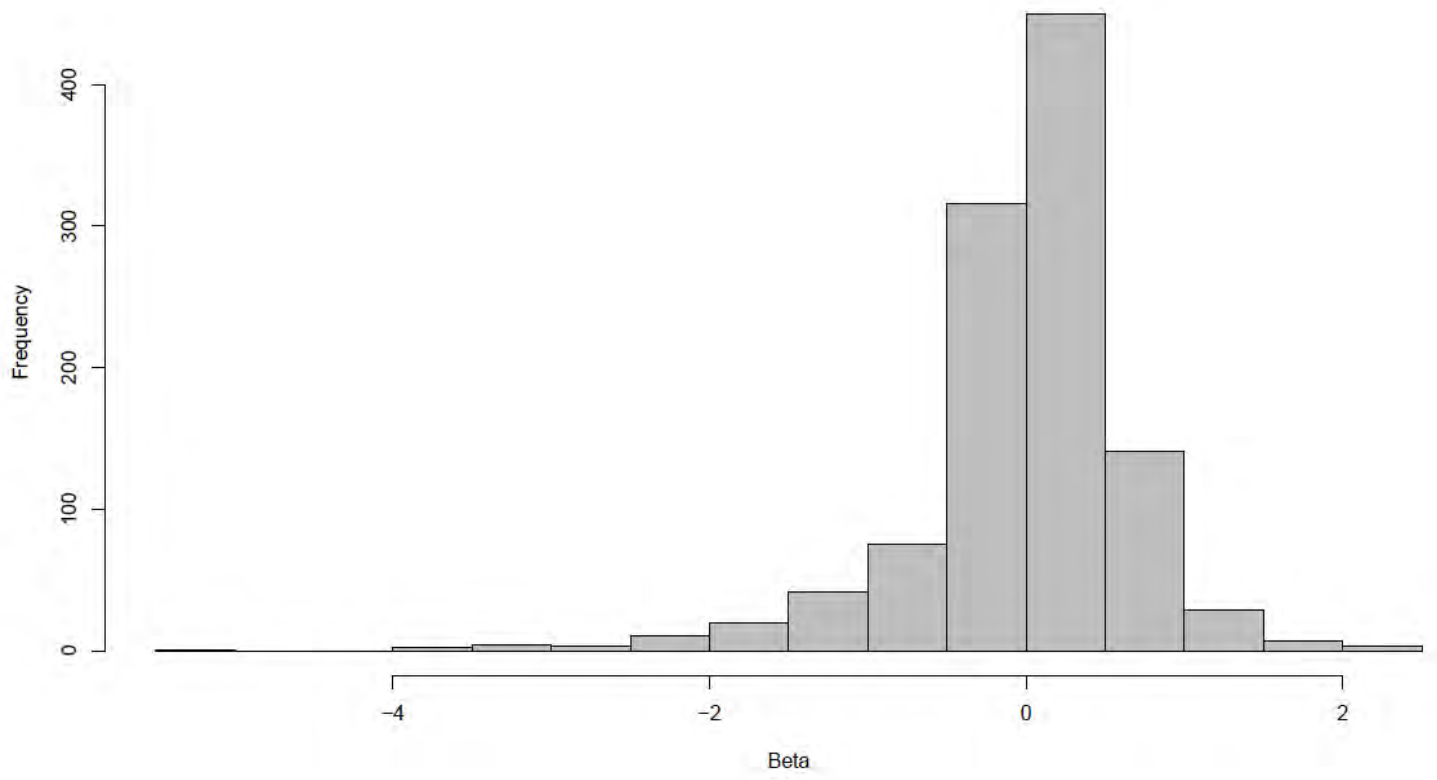

Table 6: Statistics of Green Risk Factor's Betas in different clusters

\begin{tabular}{lrrrr}
\hline & Mean & Std.Dev. & \% significant & \% positive \\
\hline Brown & -0.27 & 0.56 & 42.61 & 34.64 \\
Non-green & 0.09 & 0.35 & 17.08 & 62.60 \\
Stable green* & 0.18 & 0.24 & 36.97 & 75.77 \\
\hline
\end{tabular}

\section{Conclusions}

We identify green firms among listed companies in the European Union using a comprehensive database of environmental information at time/firm level. Unlike other studies based on subjective criteria for the choice of green firms, we propose an innovative approach that identifies a time-varying number of green firms using unsupervised learning techniques. This is the main contribution of our study to the existing literature. We then defined a pricing factor related to climate risk, calculated as the difference between the return on a portfolio of green and brown firms.

In line with recent studies, we find that green firms have under-performed non-green firms, i.e. investors are willing to buy green securities even if they yield lower returns (although they have higher returns than brown firms). However, focusing on the COVID-19 crisis period, we find that green firms outperformed the whole market, since they had higher returns than both brown and non-green firms. In addition, we also find that during the COVID-19 crisis green firms also had higher risk-adjusted return. Finally, we find that green firms are more liquid than brown and non-green firms and this gap increased during COVID-19 crisis. Overall, our results are consistent with recent contributions suggesting that green firms perform generally better in periods of market stress, in particular during the COVID-19 market collapse.

We then study the role of climate risk in equity prices by estimating an extended standard asset pricing model on single stocks by including the Green risk factor. For the EU stock market, our findings confirm that this factor is relevant when introduced in standard asset pricing models. Indeed, it has significance levels not far from other well-known factors in the literature (HML 
and SMB). We also find that generally green firms have positive exposure to the green risk factor (they benefit when the market concern on climate risk increases) while the opposite holds for brown firms. However, we also find that there is a significant fraction of firms whose exposure to the green risk factor is not consistent with cluster classification. This suggests that market prices might incorporate additional information on climate exposure of firms that are not correctly measured by the environmental data used to identify the clusters of firms.

More can be done to extend this approach and refine these results. Firstly, in this paper we clustered firms using only five different indicators, and by relying heavily on the environmental scores produced by Refinitiv. An alternative approach could be to cluster firms directly using the KPI used in the analysis, thus considering a larger set of information and without relying on the aggregation method used by the data providers in creating the scores. Secondly, we find that green firms are usually bigger (in terms of market value) than non-green and brown firms. This might be explained by the fact that big firms are better equipped to disclose large amount of information and therefore they receive higher scores. To account for this bias a potential solution could be to apply clustering techniques to separate green firms in two separate groups of firms: large cap and smallmid cap firms and take the union of these two sets. Another potential criticism is related to the definition of brown firms, which are identified as firms that belong to Climate Policy Relevant Sectors.

However, not all brown firms are the same. Brown activities are still needed in the transition to a greener economy and some of these firms could have started investment plans to reduce their emissions or to diversify their activity. A way to overcome this criticism would be to apply the clustering procedure to CPRS firms and identify "truly brown" firms as the worst performers among the clusters identified by the algorithm. Finally, we find that not all green firms have positive exposure to the green risk factor (and similarly for brown firms) suggesting that market prices might incorporate additional information on climate exposure that is not incorporated in our data. Integrating this information in our procedure would require to run clustering techniques in two steps by integrating exposure to the green risk factor for each firm in the database.

Giovanni Maria Bonagura, Luca D’Amico, Alessio Iacopino, Lorenzo Prosperi, Lea Zicchino 


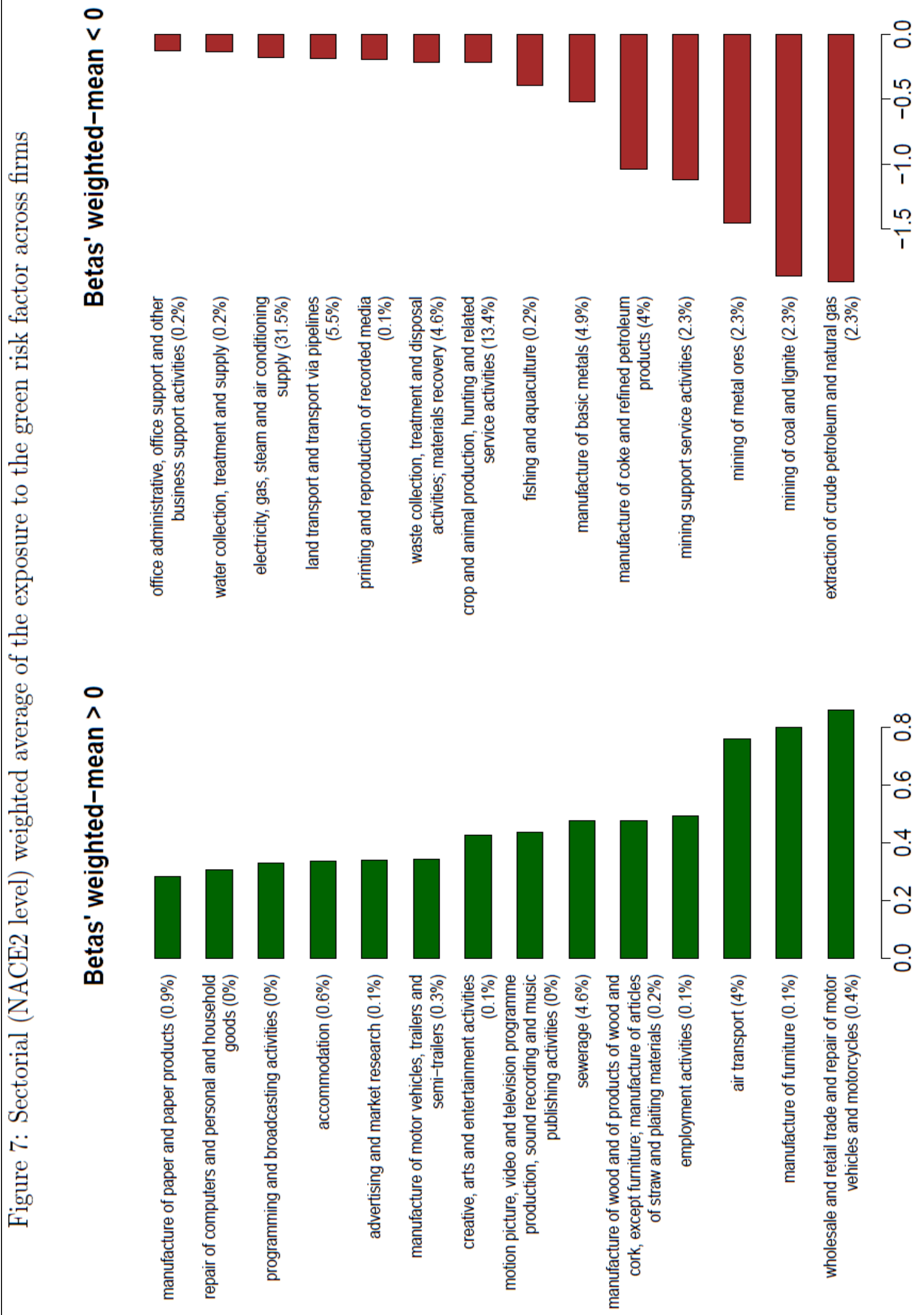




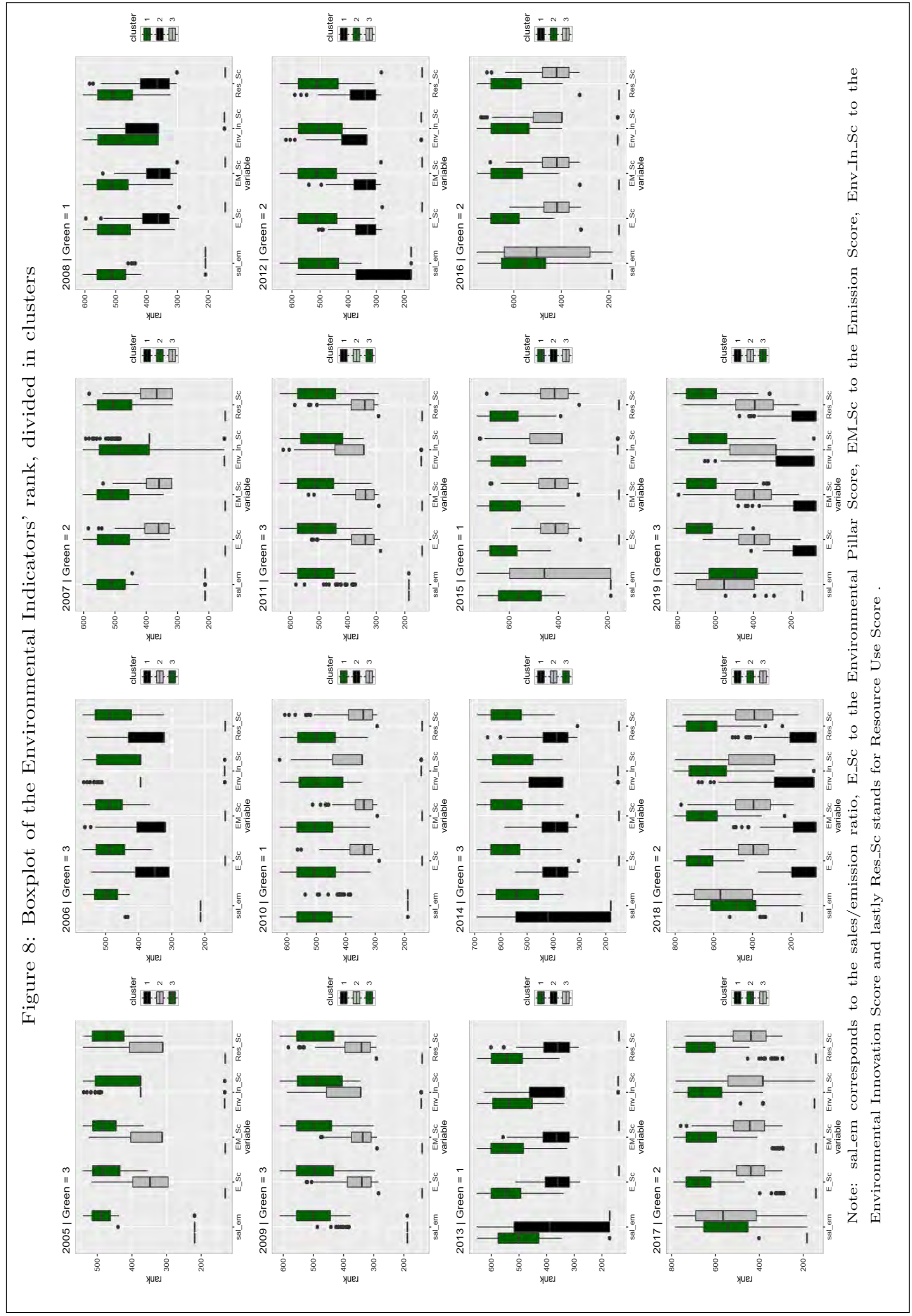


초

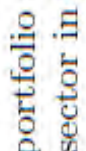

듈

5

ิㅗํ

量
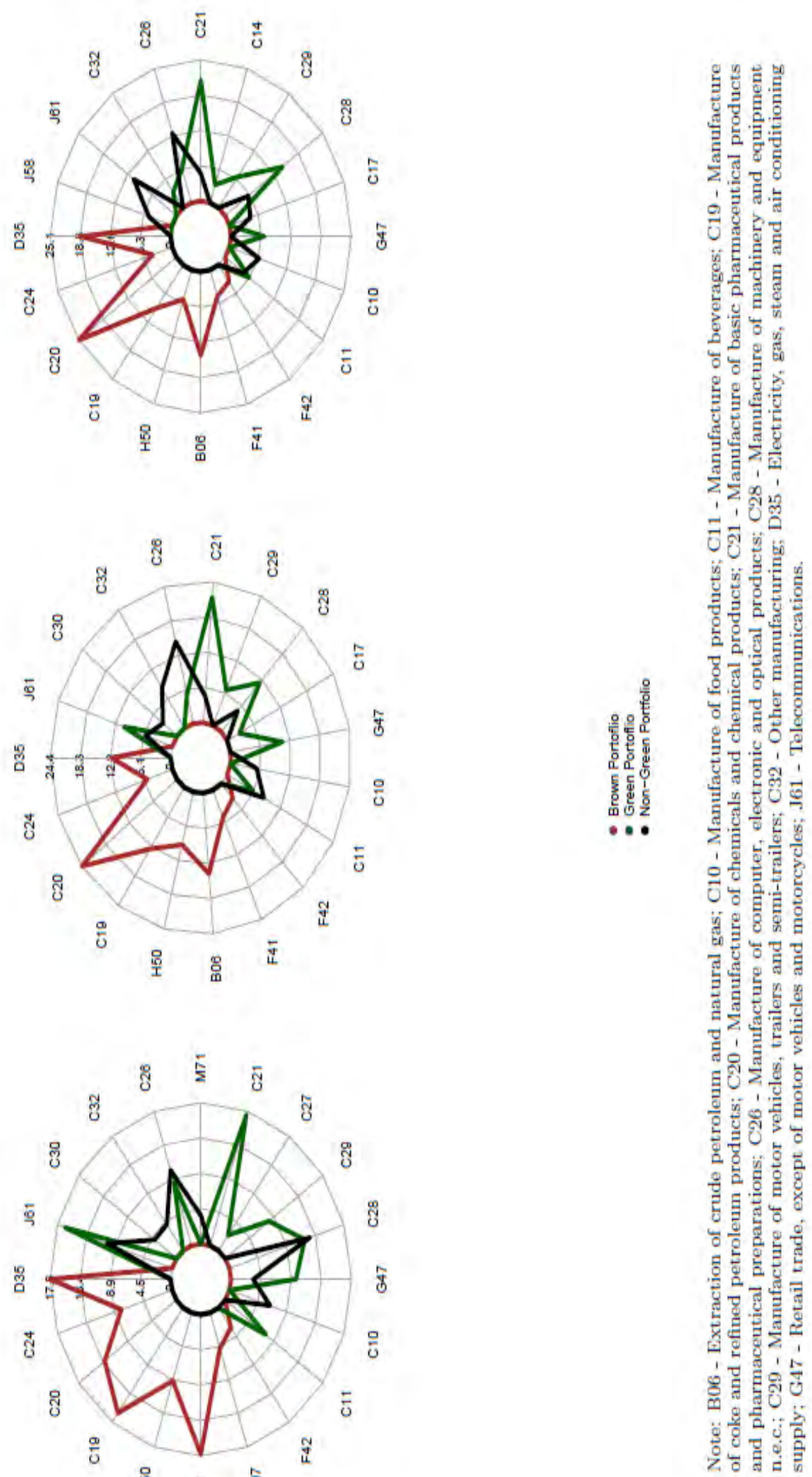


\begin{tabular}{|c|c|c|c|c|c|c|c|c|c|c|c|c|c|c|c|c|}
\hline & 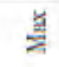 & gi gi & 옴 & $\overrightarrow{\text { g }}$ & gु & ซुg & हैं & ga & gू & g & 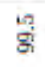 & ğ & $\begin{array}{l}\infty \\
\text { gi }\end{array}$ & $\begin{array}{l}\infty \\
g \\
g\end{array}$ & $\begin{array}{l}\infty \\
\text { g }\end{array}$ & $\begin{array}{l}\infty \\
\text { g }\end{array}$ \\
\hline 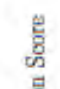 & $\begin{array}{l}\text { J } \\
\text { 总 }\end{array}$ & 。 & $=$ & 是 & $\frac{2}{60}$ & 18 & 8 & ? & 8 & $\ddot{z}$ & $\overrightarrow{\mathrm{g}}$ & 18 & $\frac{9}{3}$ & $\frac{\pi}{3}$ & ț & $\frac{9}{3}$ \\
\hline 焉 & 률 & Jี & $\stackrel{9}{g}$ & 음 & नี & से & के & $\vec{g}$ & $\overrightarrow{5}$ & $\stackrel{m}{5}$ & 9 & 돈 & p) & 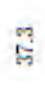 & 2 & 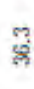 \\
\hline 变 & 量 & $=$ & $=$ & $=$ & $\vec{a}$ & $\frac{13}{N}$ & $\ddot{d}$ & $\tilde{\tilde{\sigma}}$ & กิ่ & ్ํํ & 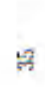 & $\vec{\phi}$ & 量 & $\$$ & 离 & $\frac{9}{3}$ \\
\hline 意 & 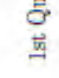 & - & - & 0 & 。 & 0 & 0 & 0 & 0 & 0 & o & 0 & - & 0 & 0 & 0 \\
\hline & 县 & 。 & $=$ & 0 & 。 & $=$ & 。 & 。 & $=$ & 0 & $=$ & 0 & o & - & $=$ & $=$ \\
\hline & $\stackrel{\ddot{\alpha}}{z}$ & 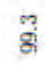 & g & $\overrightarrow{8}$ & \& & है & 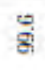 & 8 & gi & ริ & है & \& & $\stackrel{\infty}{\xi}$ & $\stackrel{\infty}{8}$ & 8 & $\stackrel{\infty}{g}$ \\
\hline & $\begin{array}{l}\text { 总 } \\
\text { 袁 }\end{array}$ & 당 & $\stackrel{\infty}{\stackrel{\infty}{R}}$ & तै & $\mathscr{m}$ & 量 & $\overrightarrow{8}$ & $\underset{\substack{n \\
\infty}}{0}$ & $\stackrel{2}{\infty}$ & $\ddot{8}$ & 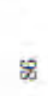 & $\underset{z}{*}$ & $\overrightarrow{8}$ & 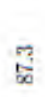 & \% & s. \\
\hline 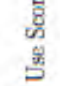 & 量 & $\begin{array}{l}\infty \\
\dot{8} \\
\dot{m}\end{array}$ & $\frac{9}{15}$ & है & 咅 & $\frac{y}{g}$ & 혐 & $\overparen{8}$ & $\widetilde{G}$ & $\overrightarrow{\mathrm{d}}$ & $\stackrel{\infty}{8}$ & $\overline{\vec{b}}$ & c. & वृ & 啳 & $\overrightarrow{8}$ \\
\hline है: & 㡺 & ने & $\vec{~}$ & 음 & 츙 & $\mathbb{B}$ & : & 思 & 름 & $\overrightarrow{\mathrm{E}}$ & 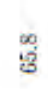 & 苗 & $\ddot{0}$ & 망 & $\frac{10}{3}$ & 9 \\
\hline & $\begin{array}{l}\text { 官 } \\
\text { 国 }\end{array}$ & 。 & o & $\bar{\nabla}$ & 갤 & 갈 & 용 & $\vec{g}$ & $\vec{z}$ & $\stackrel{\leftrightarrow}{\ddot{~}}$ & 莺 & $\stackrel{\infty}{\exists}$ & 管 & gु & gु & 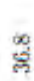 \\
\hline & 是 & 。 & $=$ & - & 。 & $=$ & $\circ$ & 。 & 0 & $\therefore$ & $=$ & 0 & $\circ$ & 。 & 0 & $\circ$ \\
\hline & $\frac{z}{2}$ & ชิ & $\overline{8}$ & $\overrightarrow{\mathrm{s}}$ & \& & 8 & s̆ & $\mathrm{g}$ & 8 & $\stackrel{\infty}{8}$ & $\stackrel{\infty}{\infty}$ & gi & $\frac{\infty}{8}$ & $\stackrel{\infty}{\S}$ & 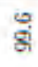 & $\stackrel{\infty}{8}$ \\
\hline & $\begin{array}{l}\text { 号 } \\
\text { 芦 }\end{array}$ & $\overrightarrow{\mathrm{d}}$ & ?ֶ: & 量 & 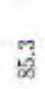 & $\frac{9}{10}$ & 5 & $\ddot{\ddot{x}}$ & $\frac{9}{x}$ & $\frac{3}{\infty}$ & $\ddot{g}$ & 咅 & $\frac{3}{40}$ & $\tilde{s}$ & $\overrightarrow{\vec{x}}$ & 量 \\
\hline 思 & 遭 & 荫 & 웍 & 萌 & $\begin{array}{l}\infty \\
0 \\
\text { 量 }\end{array}$ & 总 & 웅 & 암 & 䈢 & द्व & : & 这 & 웡 & 경 & 5 & 잉 \\
\hline 置 & $\begin{array}{l}\text { 㞼 } \\
2\end{array}$ & ్ㅓㅇ & 혐 & 7 & 6 & 挏 & 8 & 영 & J & $\infty$ & 형 & $\frac{\pi}{8}$ & 당 & 是 & है & $\frac{9}{3}$ \\
\hline & $\begin{array}{l}\dot{g} \\
\mathbb{z}\end{array}$ & $=$ & $=$ & 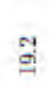 & ले & 号 & 占 & ק्में & $\overrightarrow{\mathrm{g}}$ & $\stackrel{7}{F}$ & है & 巳ே. & 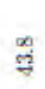 & gु & 羿 & $\stackrel{\text { gh }}{g}$ \\
\hline & 典 & 。 & 0 & o & o & 0 & o & 0 & - & $=$ & 。 & 0 & - & o & 0 & 0 \\
\hline & 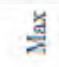 & 굼 & 8 & gु & gू & \& & gु & $\ddot{\alpha}$ & 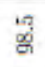 & 염 & $\bar{g}$ & $\stackrel{5}{5}$ & $\mathscr{g}$ & $\overrightarrow{\mathrm{g}}$ & $g$ & g영 \\
\hline है & $\begin{array}{l}\text { 总 } \\
\text { 䞶 }\end{array}$ & 落 & 곰 & $\overrightarrow{\mathrm{G}}$ & m & 18 & 昆 & $\widehat{t}$ & $\stackrel{\varphi}{\vec{E}}$ & 皇 & 官 & $\overrightarrow{8}$ & $\ddot{*}$ & $\tilde{R}$ & $\vec{Z}$ & $\stackrel{\substack{i \\
\tau}}{t}$ \\
\hline 急 & 昜 & $\stackrel{10}{8}$ & $\overrightarrow{\text { हु }}$ & 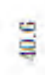 & $\stackrel{\circ}{\mathrm{g}}$ & 3 & 겸 & $\ddot{2}$ & 8 & ) & $\bar{x}$ & 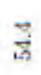 & $\frac{\infty}{a j}$ & d & & $\overrightarrow{8}$ \\
\hline 要 & 量 & สู่ & '气 & ฮี & $\stackrel{0}{g}$ & 总 & ํㅐㅁ & $t_{i}$ & 염 & 䈏 & '气 & कृ & 离 & ğ & 语 & 8 \\
\hline 总 & $\begin{array}{l}\dot{j} \\
\text { 芴 }\end{array}$ & $\rightarrow$ & $\therefore$ & $\stackrel{\overrightarrow{0}}{\sim}$ & $\tilde{H}$ & ไ్ & స్లి & 각 & 敇 & की & $\stackrel{\infty}{\vec{j}}$ & ষ্ল & $\mathrm{x}$ & के & 5 & $\mathrm{f}$ \\
\hline & 县 & 0 & $=$ & $=$ & 0 & 0 & $=$ & 0 & $=$ & 0 & o & 0 & 0 & 。 & 0 & 0 \\
\hline & 葛 & ث્户 & $\overrightarrow{\mathrm{I}}$ & ్ㅐㄹ & $\begin{array}{l}\frac{10}{2} \\
\frac{5}{5}\end{array}$ & 峦 & 곡 & 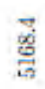 & 骇 & 啇 & 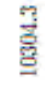 & 商 & 害 & $\begin{array}{l}\text { 总 } \\
\text { 营 }\end{array}$ & & 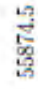 \\
\hline 足 & $\begin{array}{l}\text { Jें } \\
\text { 趈 }\end{array}$ & gु & 兽 & 롬 & 喬 & $\vec{J}$ & 官 & 兽 & ${ }^{\infty}$ & $\underset{\pi}{i}$ & స్ & $\vec{\sigma}$ & $\stackrel{\infty}{\xi}$ & $\Xi$ & 걱 & $\stackrel{\mathscr{g}}{7}$ \\
\hline 品 & $\frac{\vec{E}}{2}$ & 혹 & \& & $\underset{\aleph}{\infty}$ & 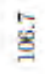 & 离 & ర్త, & $\vec{g}$ & 염 & ํㅐㅁ & $\overrightarrow{\mathrm{g}}$ & $\underset{\exists}{\exists}$ & $\stackrel{\infty}{\mathbf{s}}$ & సี & 5 & 言 \\
\hline$\frac{5}{\frac{0}{n}}$ & 善 & $\begin{array}{l}\infty \\
\stackrel{f}{g}\end{array}$ & $\stackrel{g}{0}$ & 룰 & $\overline{9}$ & $\overrightarrow{\mathrm{g}}$ & ปี & $\vec{~}$ & सें & స્ & శ్며 & $\underset{\pi}{\infty}$ & gे & 갰 & नें & $\vec{m}$ \\
\hline & 总 & $\overrightarrow{\mathrm{i}}$ & 의 & $\hat{\mathrm{m}}$ & $\ddot{m}$ & ${ }_{\tilde{f}}^{\infty}$ & $\stackrel{\circ}{\circ}$ & 5 & 3 & $\ddot{B}$ & $\overrightarrow{0}$ & to & $\stackrel{0}{0}$ & $\begin{array}{c}\infty \\
0\end{array}$ & or & 영 \\
\hline & 是 & 。 & $\overrightarrow{0}$ & $\circ$ & 。 & $\overrightarrow{0}$ & 。 & 。 & 0 & 0 & $=$ & 0 & 。 & $\overrightarrow{0}$ & 0 & 。 \\
\hline & 苟 & 兽 & 8 & 氮 & 总 & 월 & ํำ & $\overrightarrow{\text { ज्ञ }}$ & 열 & בั & 茫 & 量 & בั & 氙 & 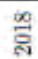 & 굴 \\
\hline
\end{tabular}




\section{References}

- $\quad$ Albuquerque, R., Y. Koskinen, S. Yang, and C. Zhang (2020). Resiliency of environmental and social stocks: An analysis of the exogenous covid-19 market crash. The Review of Corporate Finance Studies.

- $\quad$ Alessi, L., E. Ossola, and R. Panzica (2020). The greenium matters: greenhouse gas emissions, environmental disclosures, and stock prices. European Commission, Joint Research Centre, Working Papers in Economics and Finance, 2019/12.

- $\quad$ Berg, F., J. F. Koelbel, and R. Rigobon (2020). Aggregate confusion: the divergence of esg ratings. Available at SSRN 3438533.

- Bolton, P. and M. Kacperczyk (2020a). Do investors care about carbon risk? Technical report, National Bureau of Economic Research.

- $\quad$ Bolton, P. and M. T. Kacperczyk (2020b). Carbon premium around the world.

- Briére, J.-J. B. M. (2020). Esg resilience during the covid crisis: Is green the new gold? Technical report, European Capital Market Institute.

- Fama, E. F. and K. R. French (1993). Common risk factors in the returns on stocks and bonds.

- Ferriani, F. and F. Natoli (2020). Esg risk in times of covid-19. Bank of Italy Covid19 Notes.

- Gagliardini, P., E. Ossola, and O. Scaillet (2016). Time-varying risk premium in large cross-sectional equity data sets. Econometrica 84(3), 985-1046.

- $\quad$ Goodwin, T. H. (1998). The information ratio. Financial Analysts Journal 54(4), 34-43.

- Görgen, M., A. Jacob, M. Nerlinger, R. Riordan, M. Rohleder, and M. Wilkens (2019). Carbon risk. Available at SSRN 2930897.

- $\quad \operatorname{IMF}(2020$, April). Global financial stability report: Markets in the time of covid-19. Technical report, International Monetary Fund.

- Kaplan, P. D. and J. A. Knowles (2004). Kappa: A generalized downside risk-adjusted performance measure. Journal of Performance Measurement. 8, 42-54.

- Malika, C., N. Ghazzali, V. Boiteau, and A. Niknafs (2014). Nbclust: an r package for determining the relevant number of clus ters in a data set. J. Stat. Softw 61, 1-36.

- Nofsinger, J. and A. Varma (2014). Socially responsible funds and market crises. Journal of Banking \& Finance 48, $180-193$.

- $\quad$ Pastor, L., R. F. Stambaugh, and L. A. Taylor (2019). Sustainable investing in equilibrium. Technical report, National Bureau of Economic Research.

- Pastor, L. and M. B. Vorsatz (2020). Mutual fund performance and flows during the covid-19 crisis. Technical report, National Bureau of Economic Research.

- $\quad$ Sarr, A. and T. Lybek (2002). Measuring liquidity in financial markets, Volume 2. International Monetary Fund.

- $\quad$ Sharpe, W. F. (1994). The sharpe ratio. Journal of portfolio management 21(1), 49-58.

- $\quad$ Sortino, F. A. and L. N. Price (1994). Performance measurement in a downside risk framework. the Journal of Investing 3(3), 5964.

- $\quad$ Treynor, J. (1965). How to rate management of investment funds.

- Treynor, J. L. and F. Black (1973). How to use security analysis to improve portfolio selection. The journal of business 46(1), 6686.

\section{Appendix A}

Refinitiv Environmental Indicators' definition, as defined by Refinitiv:

- Sales/Emission Ratio: Net sales or revenue in US dollars divided by Total CO2 and CO2 equivalents emission in tonnes.

- Environmental Pillar Score: The environmental pillar measures a company's impact on living and non-living natural systems, including the air, land and water, as well as complete ecosystems. It reflects how well a company uses best management practices to avoid environmental risks and capitalize on environmental opportunities in order to generate long term shareholder value.

- Emission Score: Emission category score measures a company's commitment and effectiveness towards reducing environmental emission in the production and operational processes

- Resource Use Score: Resource use category score reflects a company's performance and capacity to reduce the use of materials, energy or water, and to find more eco-efficient solutions by improving supply chain management.

- Environmental Innovation score: Environmental innovation category score reflects a company's capacity to reduce the environmental costs and burdens for its customers, and thereby creating new market opportunities through new environmental technologies and processes or eco-designed products. 


\section{Climate Change: EU taxonomy and forward looking analysis in the context of emerging climate related and environmental risks}

Giuliana Birindelli (G. d'Annunzio University of Chieti-Pescara), Vera Palea (University of Turin), Luca Trussoni (LTlogics), Fabio Verachi (Intesa Sanpaolo)

Article submitted to double-blind peer review, received on 14th September 2020 and accepted on 24th November 2020

\section{Abstract}

Climate change is causing substantial structural adjustments to the global economy. Several sectors, such as coal and steel, are undergoing severe problems related to the inevitable transition to a low-carbon economy, while others such as renewables and new environmental adaptation technologies are benefiting substantially. In this context, regulators are beginning to intervene on the legislation, while investors, customers and civil society are looking for alternatives to mitigate, adapt and make these issues more transparent. This article aims to analyze the impact that these changes will inevitably have on banks' balance sheets, introducing new risks but also opportunities. The final purpose is to help banks integrate climate risks into their organizational framework and to provide guidance on the implementation of the recommendations published by the Task Force on Climaterelated Financial Disclosures (TCFD) within the broader Financial Stability Board (FSB) objectives and the UN Environment Finance Initiative (UNEP FI). Starting from a long-term perspective, the work suggests considering climate risk as a financial risk, overcoming traditional approaches that focus on reputational risk. This change implies the integration of climate change risk into the logic of Risk Management (Credit, Market and Operational risks) and a consequent sharing of responsibilities with the structures of Corporate Social Responsibility (CSR). The TCFD recommendations urge banks to use forward looking scenario analyzes, including stress tests, to evaluate and disseminate the "actual and potential impacts" of climate-related risks and opportunities, suggesting in particular to consider the consequences in terms of two categories of risk: physical and transition risk.

Keywords: "climate change", "transition risk", "physical risk", "EU taxonomy", "IPCC scenarios", "RCP-SSP-SPA pathways", "forward looking analysis", "climate stress test", "PD assessment"

\section{Introduction to climate change risk}

Climate change implies significant economic costs. The most common are damage caused by extreme weather events such as storms or floods. Further examples are disruptions in supply chains, higher prices as a result of shortages due to drought or lower labor productivity in case of severe heat waves. These events, better known as physical risks, are already affecting our economies and scientists agree that they will increase over time.

On the other hand, the transition to a low-carbon economy, necessary to mitigate these costs, has economic and social consequences: investments in low-carbon technologies and higher carbon prices combined with a possible carbon tax will reduce margins with the consequence that some polluting activities will have to be dismissed. It is likely that these phenomena, known as transition risk, will be substantial and must be managed with an unprecedent attention.

Physical and transition risks, in addition to their direct impact in terms of reducing the value of tangible and intangible assets (market risk, technological risk and reputational risk), will ultimately result in higher expenses and lower revenues, or will reduce cash flows of the Corporate, SME and Retail segments. Lower cash flows and lower asset values, as well as their volatility, are key determinants in assessing financial robustness and therefore the ability to repay debts, with obvious implications in the creditworthiness. As a consequence, physical and transition risk are a source of credit risk, namely the climate change credit risk.

Capasso et al. (2020) [7] indeed show that the exposure to climate change decreases firms' distance to default. This implies an increase in banks' asset value at risk. Battiston et al. (2017) [6] indicate that, in the Euro Area, the bank exposures to climatepolicy relevant sectors are large, heterogeneous, and possibly amplified by indirect exposures via financial counterparties. Thus, the exposure to climate risk could potentially pose systemic threats to global financial stability. Demtz et al. (2016) [14] estimate that, in a business as usual scenario, the climate value at risk would be around 2.5 trillion dollars.

This work aims to be a reference tool for risk managers of the financial industry that need to deepen the methodologies for managing climate change risk: it focuses on the relevant aspects from a financial point of view, providing an overview of the literature and illustrating some quantitative tools useful for the risk assessment. The data and estimates presented are purely illustrative and informative: they are largely taken from scientific works and public databases that do not take into account, as they were previously produced, the economic impact and consequences on the markets generated by the Covid-19 pandemic. However, before introducing the analysis of the methods for assessing climate risks, it is useful to provide some indications on the action plan adopted in this context by the European Commission and, in particular, on the methods of identifying sustainable activities (so-called taxonomy).

The structure of the paper is as follows. Section 2 deals with the taxonomy mentioned above, Section 3 focuses on issues related to climate risk assessment, Sections 4 and 5 explore the issue of climate scenarios, Section 6 focuses on transition risk approaches, Section 7 on the implications of transition risk on creditworthiness measurement, Section 8 on the impact of physical risk on creditworthiness. Finally, Section 9 concludes. 


\section{EU taxonomy of "sustainable" financial products}

The European Commission, as part of the action plan for sustainable finance, has promoted a series of activities including the mandate to the Technical Expert Group on Sustainable Finance (TEG) to develop a unique classification within the EU, the socalled taxonomy of economic activities that can be considered sustainable. The development of this classification, based on technical-scientific definitions, aims to guarantee the reliability and comparability of information on sustainable investments, promote transparency and long-term vision and discourage the phenomenon of greenwashing through the adoption of a common language.

The taxonomy does not constitute a list of activities to invest in, nor a classification system for the quality of businesses or a list of activities that must excluded. Instead, it represents a list of economic activities to which performance criteria are associated to evaluate the contribution with respect to the environmental objectives identified by the European community:

1. climate change mitigation;

2. climate change adaptation;

3. $\quad$ sustainable use and protection of water and marine resources;

4. transition to a circular economy, waste prevention and recycling;

5. prevention and control of pollution;

6. protection and safety of ecosystems.

To be included in the taxonomy, an economic activity must: (i) contribute significantly to at least one of the environmental objectives (respecting specific technical criteria, metrics and thresholds); (ii) not significantly harm the other objectives (Do No Significant Harm - DNSH); (iii) comply with the "minimum social standards". The technical evaluation criteria can include qualitative or quantitative thresholds (often expressed in terms of $\mathrm{CO} 2$ emissions), representative of the environmental performance objectives expected from the economic activities under exam.

In line with the mandate of the European Commission, the work of the TEG on taxonomy initially focused on climate change objectives. The reports on the new EU taxonomy published by the European Commission in March 2020 [32] therefore focus on the first two environmental objectives and on the activities that can provide a substantial contribution to climate change mitigation and adaptation.

In particular, the new EU taxonomy analyzes the activities relating to seven macro-sectors selected on the basis of $\mathrm{CO} 2$ emissions and potential savings also in terms of "enabling technologies"1:

- $\quad$ agriculture, forestry and fishing;

- $\quad$ manufacturing;

- $\quad$ electricity, gas, steam and air conditioning;

- $\quad$ water, sewage, waste and remediation activities;

- $\quad$ transportation and storage;

- $\quad$ ICT (Information, Communication and Technology);

- $\quad$ construction and real estate activities.

With reference to the mitigation and transition objective towards a zero-emission economy, the activities included in the taxonomy can be classified into three macro-categories:

- "low carbon" economic activities characterized by zero / near-zero or negative emissions and therefore already compatible with the objectives of zero net emissions by 2050 (e.g. transport activities with electric vehicles and the production of energy from renewables);

- economic activities that contribute to the transition process which, although not close to the zero emissions target, are characterized by performances above the sector average. For these activities, the compliance with specific technical criteria and emission thresholds subject to regular revisions is required (e.g. the generation of electricity with emissions lower than $100 \mathrm{~g} \mathrm{CO} 2 / \mathrm{KWh}$ );

- other activities ("enabling activities") that allow and support the transition towards a zero-emission economy (e.g. the construction of solar panels for electricity generation and turbines for wind farms or interventions to improve the energy efficiency of buildings).

Regarding the issues of "climate adaptation", the analysis must be carried out on the basis of an assessment related to the specific context and geographical location, following three guiding principles:

1. economic activity adopts all possible measures to reduce the relevant physical risks deriving from the variability of meteorological phenomena and climate change;

2. the economic activity does not negatively impact other activities;

3. the contribution to adaptation can be identified by means of appropriate indicators.

Furthermore, in line with the EU strategy for increasing resilience to climate change, the TEG has developed a specific classification of climate risks. With reference to the importance and frequency of these events, "chronic" or "acute" effects are distinguished.

With respect to the area of relevance, the effects produced by climate change may affect: climate, temperature, winds, water and soil (see table below).

\footnotetext{
${ }^{1}$ Failure to include some activities does not automatically imply that these are harmful to the environment. In fact, some of the activities not included may have a positive marginal contribution or be neutral.
} 
Table 1 - Classification of climate change related risks and events in terms of frequency/severity

\begin{tabular}{|c|c|c|c|c|}
\hline & Temperature / climate & Winds & Water & Soil \\
\hline \multirow{4}{*}{ 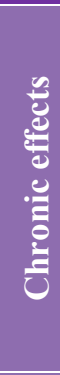 } & $\begin{array}{l}\text { Increase in average } \\
\text { temperatures (air, water) }\end{array}$ & $\begin{array}{l}\text { Change of direction } \\
\text { and intensity of winds }\end{array}$ & $\begin{array}{l}\text { Changes in intensity, } \\
\text { frequency and duration of } \\
\text { precipitation }\end{array}$ & $\begin{array}{l}\text { Coastal erosion } \\
\text { phenomena }\end{array}$ \\
\hline & Temperature variability & & $\begin{array}{c}\text { Changes in hydrogeological } \\
\text { system }\end{array}$ & Desertification \\
\hline & Ice melting / permafrost & & $\begin{array}{l}\text { Marine system modifications } \\
\text { (acidification, salinity, etc.) }\end{array}$ & \\
\hline & & & $\begin{array}{c}\text { Rise in level of seas and } \\
\text { rivers }\end{array}$ & \\
\hline \multirow{4}{*}{ 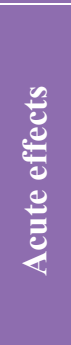 } & Intense heat waves & $\begin{array}{l}\text { Hurricanes, cyclones, } \\
\text { typhoons, windstorms } \\
\text { and tornadoes }\end{array}$ & Drought & $\begin{array}{l}\text { Landslides and } \\
\text { avalanches }\end{array}$ \\
\hline & Intense cold waves & & $\begin{array}{l}\text { Extraordinary precipitation } \\
\text { (rain, snow, hail, etc.) }\end{array}$ & \\
\hline & Fires & & $\begin{array}{l}\text { Floods (fluvial, pluvial, } \\
\text { marine) }\end{array}$ & \\
\hline & & & Melting glaciers & \\
\hline
\end{tabular}

Concerning the scope of application, the taxonomy includes:

- companies and other subjects that are included within the scope of application of the Non Financial Reporting Directive (Directive 2014/95) for the related disclosure and reporting obligations;

- financial institutions ${ }^{2}$ for identifying, evaluating and classifying sustainable financial products;

- European Community countries to define measures and requirements related to sustainable financial products.

It is therefore reasonable to expect that the availability of a reference taxonomy promoted at EU level will have a significant effect in terms of standardization and convergence of the methodologies adopted by other agents, including non-EU ones. In operational terms, the application by investors must be divided into the following steps:

- identify the financed activities carried out by a company (or a project) to assess their consistency with the taxonomy;

- for each activity, check whether the criteria, metrics and thresholds indicated by the taxonomy are met (i.e. compliance with the $\mathrm{CO} 2$ emission thresholds);

- verify, through a due diligence process, compliance with the Do Not Significant Harm (DHSH) criteria;

- verify compliance with the "minimum social standards"3;

- prepare, after verifying the alignment of the investment with the taxonomy, the correct information at the product level.

Finally, it should be noted that the taxonomy will be implemented in the EU through delegated acts by December 2020, with entry into force expected in December 2021.

\section{How to assess climate risks? From building climate scenarios to measuring financial impacts}

The tools for assessing climate risks from a financial perspective are in an experimental phase and only a few studies provide an integrated and comprehensive overview ${ }^{4}$. This work proposes a framework based on forward looking stress tests for the analysis of physical and transition risk related to different climate scenarios. The authors tried to align with the stress test best practices (Basel Committee on Banking Supervision, 2018). This implies that these methodologies can, in principle, be applied by banks both in the bottom-up stress tests and in the top-down exercises proposed by the supervisors (e.g. EU-wide stress test proposed by EBA).

To assess the impact of climate change risks, a building block approach is therefore proposed with methodologies that can be mainly divided into three phases:

1. definition of climate scenarios: the estimate of the climate change impact is primarily based on the definition of forward looking scenarios. These scenarios define how climate change will impact the variables relevant for the economic activities, how a transition will mitigate those impacts, and what measures could be taken to steer the transition;

2. estimation of the economic and financial impacts: once the impact of climate change has been estimated, its consequences must be translated into financial terms through macro and microeconomic simulations. This step essentially evaluates the direct and indirect effects of climate change, the transition modalities and identifies which actors are affected and to what extent;

\footnotetext{
${ }^{2}$ Including, for example: asset managers and investment fund management companies (with underlying equities, ETF bonds), real estate funds, private equity and venture capital funds, alternative investment funds, infrastructure funds, funds of funds, etc.

${ }^{3}$ Alignment with the Minimum Social Safeguard allows to grasp the social and governance aspects by verifying, on the basis of a due diligence, compliance with international legislation on human, job and anti-corruption rights (e.g. OECD Guidelines on Multinational Enterprises and for Responsible Business Conduct [26], UN Guiding Principles on Business and Human Rights, International Labor Organizations [37]).

${ }^{4}$ Fundamental works on the subject, just to name a few, are Monnin (2018) [25], UNEP FI - OW/Acclimatise (2018) [29], Jansen (2019) [23] and DNB (2019) [40].
} 
3. transformation of financial impacts into risk measures: based on the assessment of the impacts, the next step is to calculate how changes in cash flows and balance sheets will affect the various risk measures (e.g. market values of assets, creditworthiness in terms of rating and probability of default).

\section{Climate change scenarios}

The first tool for modeling climate change (and therefore also the related risks) is the development of specific scenarios. Since 1992, the Intergovermental Panel on Climate Change (IPCC) built a first set of scenarios, then revised in 2000 when the IPCC released the Special Report on Emissions Scenarios (SRES), proposing a set of 40 scenarios organized into 4 families (see for example [20], the following description is taken from [17]):

A1: this family of scenarios describes a future with very rapid economic growth, the global population will have a maximum until 2050 and then decrease, and a rapid introduction of new and more efficient technologies. This family is developed into three groups that describe alternative directions in the technological changes of the energy system: $\underline{\mathrm{A} 1 \mathrm{Fl}}$ future with fossil fuels, $\underline{\mathrm{A} 1 \mathrm{~T}}$ non-fossil resources, $\underline{\mathrm{A} 1 \mathrm{~B}}$ equilibrium between fossil fuels and other sources;

A2: this scenario describes a very heterogeneous world. There will be a continuous demographic increase with a per capita economic growth. Technological changes will be very fragmented and slow;

B1: this scenario as well foresees that the population growth will reach its peak in the middle of the century and then decline, but a rapid evolution towards an information and services economy, with a reduction of materials and the introduction of new technologies, will be capable of generating efficient and clean resources;

B2: the population is growing continuously, but at a lower rate than the A2 family. The economic development will reach intermediate levels with slow and differentiated technological changes, but always oriented towards sustainable development.

Subsequently, starting from 2007, in response to the need of improving the SRES, the IPCC approach has changed and turned towards the development, not anymore of a complete set of scenarios, but rather to the "Representative Concentration Pathways" (RCP) defined in the Assessment Report 5 (AR5): RCPs are quantitative forecasts of the trend of greenhouse gases and atmospheric pollutants derived from human activities (and therefore also imply specific forecasts on the trend of global warming and land use). More specifically the RCPs (Van Vuuren et al. (2011) [38]):

1. are based on the scenarios outlined in the literature. Individually, each RCP represent a coherent description of the future;

2. describe the chemistry of the atmosphere and pollutants with a geographical and temporal basis;

3. are supported by common assumptions on a year by year time horizon;

4. contain forecasts up to 2100 .

The AR5 proposes, with the aim to arrive at an integrated description such as that proposed by the previous SRES scenarios, a matrix approach that can be combined with the RCP:

a. the Shared Socio-economic Pathways (SSP), which represent 5 possible future evolutions of the socioeconomic variables associated with the scenarios (they describe quantitative projections of gross domestic product, population, urbanization and education indexes);

b. the Shared climate Policy Assumptions (SPA), which represent the mitigation actions implemented in response to climate change, possibly defined locally.

A scenario is then fully described by the association of RCP, SSP and SPA. Comparing the literature describing the interaction between RCP and SSP (O’Neil et al. (2014) [27], O’Neil et al. (2016) [28], Rihai et al. (2017) [24]), for the purposes of this study it was decided to associate RCP and SSP on the basis of the possible overlap in the different path narratives, highlighted by the bold cells in the following table.

Table 2 - Association and choice of RCP and SSP scenarios

\begin{tabular}{|l|l|l|l|l|l|}
\hline & SSP1 & SSP2 & SSP3 & SSP4 & SSP5 \\
\hline $\boldsymbol{R C P 2 . 6}$ & Mitigation & Mitigation & Mitigation & N/A & N/A \\
\hline $\boldsymbol{R C P 4 . 5}$ & Mitigation & Mitigation & Mitigation & Mitigation & Mitigation \\
\hline $\boldsymbol{R C P 6 . 0}$ & Baseline & Mitigation & Mitigation & Mitigation & Mitigation \\
\hline $\boldsymbol{R C P 8 . 5}$ & N/A & N/A & N/A & N/A & Baseline \\
\hline
\end{tabular}

\section{Enrichment of climate scenarios with macroeconomic variables}

The previous section introduced the process of constructing future scenarios of climate change, starting from the most commonly adopted hypotheses in the literature and based on Representative Concentration Pathways (RCP) appropriately "enriched" with Shared Socioeconomic Pathways (SSP) and Shared Policy Assumptions (SPA).

Among the various possible combinations, this work has chosen to propose scenarios for the evolution of the financial system according to the classification generally adopted by the TCFD recommendations: Rapid Transition $\left(+1.5^{\circ}\right.$, global warming above pre-industrial levels), Two Degree $\left(+2^{\circ}\right.$, slight increase), Business as Intended $\left(+3^{\circ}\right.$, increase $)$ and Business as Usual $(+$ $4^{\circ}$, strong overheating).

The main parameters of the scenarios involved, with a particular focus on GDP by area (OECD the highest level of detail available), are summarized in the following tables. The authors consider appropriate to recall, once again, that the data presented 
does not incorporate the effects of the Covid-19 pandemic. However, it should be noted that the adoption of different scenarios leads to very different estimates of the main macroeconomic variables.

Table 3 - Scenario 1 RCP2.6, SSP1, SPA1 (Rapid Transition $+1,5^{\circ}$ )

\begin{tabular}{|c|c|c|c|c|c|c|c|c|}
\hline Region & Model & Variable & Unit & 2020 & 2030 & 2040 & 2050 & 2100 \\
\hline World & IMAGE - SSP1-26 & Temperature & ${ }^{\circ} \mathrm{C}$ & 1.22 & 1.48 & 1.66 & 1.76 & 1.76 \\
\hline OECD & IMAGE - SSP1-26 & Population & Million & 1,180 & 1,232 & 1,276 & 1,312 & 1,262 \\
\hline OECD & IMAGE - SSP1-26 & $\mathrm{CO} 2$ emissions & $\mathrm{Mt} \mathrm{CO} 2 / \mathrm{yr}$ & 10,653 & 8,834 & 6,837 & 4,783 & $-4,888$ \\
\hline & & OECD GDP & $\%$ yr/yr & $2.22 \%$ & $2.45 \%$ & $2.21 \%$ & $1.73 \%$ & $0.74 \%$ \\
\hline
\end{tabular}

Table 4 - Scenario 2 RCP4.5, SSP2, SPA2 (Two Degree $+2^{\circ}$ )

\begin{tabular}{|c|c|c|c|c|c|c|c|c|}
\hline Region & Model & Variable & Unit & 2020 & 2030 & 2040 & 2050 & 2100 \\
\hline World & $\begin{array}{l}\text { MESSAGE-GLOB } \\
\text { SSP2-45 }\end{array}$ & Temperature & ${ }^{\circ} \mathrm{C}$ & 1.24 & 1.49 & 1.74 & 1.97 & 2.63 \\
\hline OECD & $\begin{array}{l}\text { MESSAGE-GLOB } \\
\text { SSP2-45 }\end{array}$ & Population & Million & 1,168 & 1,215 & 1,251 & 1,279 & 1,272 \\
\hline OECD & $\begin{array}{l}\text { MESSAGE-GLOB } \\
\text { SSP2-45 }\end{array}$ & $\mathrm{CO} 2$ emissions & $\mathrm{Mt} \mathrm{CO} 2 / \mathrm{yr}$ & 10,932 & 10,739 & 10,882 & 11,104 & 3,189 \\
\hline & & OECD GDP & $\% \mathrm{yr} / \mathrm{yr}$ & $2.38 \%$ & $2.03 \%$ & $1.64 \%$ & $1.41 \%$ & $0.88 \%$ \\
\hline
\end{tabular}

Table 5 - Scenario 3 RCP6.0, SSP3, SPA3 (Business as Intended $+3^{\circ}$ )

\begin{tabular}{|c|c|c|c|c|c|c|c|c|}
\hline Region & Model & Variable & Unit & 2020 & 2030 & 2040 & 2050 & 2100 \\
\hline World & AIM/CGE SSP3-60 & Temperature & ${ }^{\circ} \mathrm{C}$ & 1.23 & 1.52 & 1.85 & 2.10 & 3.18 \\
\hline OECD & AIM/CGE SSP3-60 & Population & Million & 1,153 & 1,159 & 1,146 & 1,116 & 865 \\
\hline \multirow[t]{2}{*}{ OECD } & $\begin{array}{l}\text { AIM/CGE SSP3-60 } \\
\text { (Baseline) }\end{array}$ & $\mathrm{CO} 2$ emissions & $\mathrm{Mt} \mathrm{CO} 2 / \mathrm{yr}$ & 14,519 & 14,140 & 13,481 & 12,875 & 5,907 \\
\hline & & OECD GDP & $\% \mathrm{yr} / \mathrm{yr}$ & $2.18 \%$ & $1.40 \%$ & $0.81 \%$ & $0.46 \%$ & $-0.23 \%$ \\
\hline
\end{tabular}

Table 6 - Scenario 4 RCP8.5, SSP5 baseline (Business as Usual $+4^{\circ}$ )

\begin{tabular}{|c|c|c|c|c|c|c|c|c|}
\hline Region & Model & Variable & Unit & 2020 & 2030 & 2040 & 2050 & 2100 \\
\hline World & $\begin{array}{l}\text { IMAGE } \\
\text { SSP5 (Baseline) }\end{array}$ & Temperature & ${ }^{\circ} \mathrm{C}$ & 1.25 & 1.59 & 1.96 & 2.38 & 4.86 \\
\hline OECD & $\begin{array}{l}\text { REMIND-MAGPIE } \\
\text { SSP5-85 (Baseline) }\end{array}$ & Population & Million & 1,296 & 1,386 & 1,477 & 1,574 & 1,916 \\
\hline \multirow[t]{2}{*}{ OECD } & $\begin{array}{l}\text { REMIND-MAGPIE } \\
\text { SSP5-85 (Baseline) }\end{array}$ & $\mathrm{CO} 2$ emissions & $\mathrm{Mt} \mathrm{CO} 2 / \mathrm{yr}$ & 11,872 & 14,399 & 17,508 & 21,155 & 33,246 \\
\hline & & OECD GDP & $\% \mathrm{yr} / \mathrm{yr}$ & $2.62 \%$ & $3.15 \%$ & $3.78 \%$ & $2.95 \%$ & $2.10 \%$ \\
\hline
\end{tabular}

However, these scenarios must be translated into quantitative measures with reference to the geopolitical area of Italy. First of all, we could estimate the relationship between the OECD GDP evolution, contained in the IPCC scenarios, and the possible dynamics of Italy's GDP.

As example, if we want to use the data relating to the period 2010-2020, with the aim to consider the most recent Italian macroeconomic context, and by adopting a linear regression, we would arrive at the estimate of the coefficients shown below:

$$
\begin{aligned}
& Y_{i}=\beta_{0}+\beta_{1} X_{i}+\varepsilon_{i} \\
& Y_{i}=\text { Italy GDP }_{i} \\
& \beta_{0}=-1.953 \\
& \beta_{1}=1.171 \\
& X_{i}=\text { oECD GDP } \\
& \varepsilon_{i}=\text { statistical error }_{i} \\
& \theta_{\varepsilon}=1.461
\end{aligned}
$$


Starting from these long-term estimates, it is possible to hypothesize 5-year projections (2020-2025) which could be taken as a reference for the first exercises in a forward looking perspective using the stress test framework. In the case in question, it was decided to incorporate the previous estimation error mentioned $\theta_{\varepsilon}$, considering a confidence level of $96 \%$ that is consistent with the stress test used in the banking sector (so-called stress $1 / 25$, i.e. occurring once every 25 years and with probability $1 / 25=4 \%=100 \%-96 \%$ ). The table below illustrates the Italy GDP trend by incorporating the stress assumptions adopted.

Table 7 - Stress projections (2020-2025) of Italy GDP

\begin{tabular}{|c|c|c|c|c|c|c|c|}
\hline Scenario & Variable & Unit & 2021 & 2022 & 2023 & 2024 & 2025 \\
\hline $\begin{array}{l}\text { Scenario } 1 \text { RCP2.6, SSP1, SPA1 } \\
\left.\text { (Rapid Transition }+1,5^{\circ}\right)\end{array}$ & Italy GDP & $\% / y r$ & $-1.89 \%$ & $-1.35 \%$ & $-0.81 \%$ & $-0.27 \%$ & $0.27 \%$ \\
\hline $\begin{array}{l}\text { Scenario } 2 \text { RCP4.5, SSP2, SPA2 } \\
\left(\text { Two Degree }+2^{\circ}\right)\end{array}$ & Italy GDP & $\% / y r$ & $-1.77 \%$ & $-1.29 \%$ & $-0.82 \%$ & $-0.35 \%$ & $0.12 \%$ \\
\hline $\begin{array}{l}\text { Scenario } 3 \text { RCP6.0, SSP3, SPA3 } \\
\text { (Business as Intended }+3^{\circ} \text { ) }\end{array}$ & Italy GDP & $\% / y r$ & $-2.05 \%$ & $-1.63 \%$ & $-1.21 \%$ & $-0.79 \%$ & $-0.37 \%$ \\
\hline $\begin{array}{l}\text { Scenario } 4 \quad \text { RCP8.5, } \\
\text { (Business as Usual }+4^{\circ} \text { ) }\end{array}$ & Italy GDP & $\% / y r$ & $-1.38 \%$ & $-0.81 \%$ & $-0.24 \%$ & $0.34 \%$ & $0.91 \%$ \\
\hline
\end{tabular}

The last step concerns the complete construction of the scenarios with the extension of the projections, in addition to the GDP of each geographical area, to the other macroeconomic variables useful for the completion of the stress exercises. In the following sections, we will show the underlying assumptions and the multi-country GVAR model, which represents one of the examples we could refer for the development of different climate scenarios, will be introduced.

\section{Overview of transition risk approaches: macroeconomic models, Top-Down and Bottom-Up methodologies}

As widely discussed, scenario analyzes require all the macroeconomic variables that represent the input of any macro and microprudential stress test exercise.

Therefore, the declination of the 4 scenarios mentioned above requires a complete narrative which, according to the indications of the literature, can be based on various hypotheses around the two risk factors that emerge as the main drivers of transition risk: the so-called climate policies adopted by regulators and any technological developments.

The last scenario is an exception (so-called Business as Usual), in which the energy transition is postponed, and technological discoveries are limited or absent: in this case, the hypotheses only take into consideration a strong decline in consumption and investor confidence.

Another aspect already mentioned concerns the time horizon of the analyzes. The scenarios must be defined in such a way that they materialize within five years, thus ensuring that the results of the climate stress tests are relevant to both financial institutions, regulators and other stakeholders. It should be emphasized that physical risks could also insist on the same scenarios, but phenomena such as floods, tornadoes or earthquakes will have to be treated separately ${ }^{5}$.

With this in mind, to translate each scenario into a series of macroeconomic impacts, we can make reference to macroeconometric models, such as those known as multi-country Global Vector AutoRegressive (GVAR) ${ }^{6}$. The use of this type of macroeconometric models provides several advantages.

Firstly, they allow a simulation of a series of mutually correlated macroeconomic impacts that can serve as inputs to top-down stress test models. Secondly, given the strong correlations in the markets, it is possible to measure the spillover effects between various countries and between various sectors.

On the other hand, there are also strong limitations. These models, based on long-term historical data, are not designed to simulate structural economic breaks that can be triggered by entirely new phenomena, such as the transition to a low-carbon economy.

Once the impacts on the main macroeconomic variables have been estimated, it remains to understand how to transform them into changes in risk measures of the economic activities.

As shown in the figure, there are different methodological approaches on the topic and, obviously, each method offers a compromise between feasibility and analytical rigor. The methodologies can be basically summarized in 2 macro categories:

- top-down approaches: the risk deriving from the variations in country-level macroeconomic variables is first calculated at sectoral level and then disaggregated referring to specific indicators (e.g. CO2 emissions);

- bottom-up approaches: the analysis takes place directly at the individual borrower level, based on the financial figures that affect the counterparty creditworthiness.

\footnotetext{
${ }^{5}$ The impact of these scenarios on physical risks could be very significant for financial institutions. Some papers from the Bank of Italy have estimated, for example, that the flooding risk could result in substantial losses for the Italian financial system (Faiella, Natoli, 2019 [18]).

${ }^{6}$ For analytical details on the GVAR methodology, refer to Pesaran, Schuermann \& Weiner (2004) [31], Mauro \& Pesaran (2013) [15] and Barbanti Brodano, Cocco \& Moramarco (2014) [5].
} 
Figure 1 - Transition risk: general overview and main approaches

\begin{tabular}{|l|l|}
\hline $\begin{array}{c}\text { Transition Scenario } \\
\text { Sector-level Sensitivity }\end{array}$ & $\begin{array}{c}\text { Top - Down } \\
\text { Approach }\end{array}$ \\
\hline $\begin{array}{l}\text { Overall impact at } \\
\text { sector level }\end{array}$ & $\begin{array}{l}\text { Recalibration } \\
\text { Recalibration of the overall impact at sector } \\
\text { level based on the relevance of the single } \\
\text { borrower }\end{array}$ \\
\hline
\end{tabular}

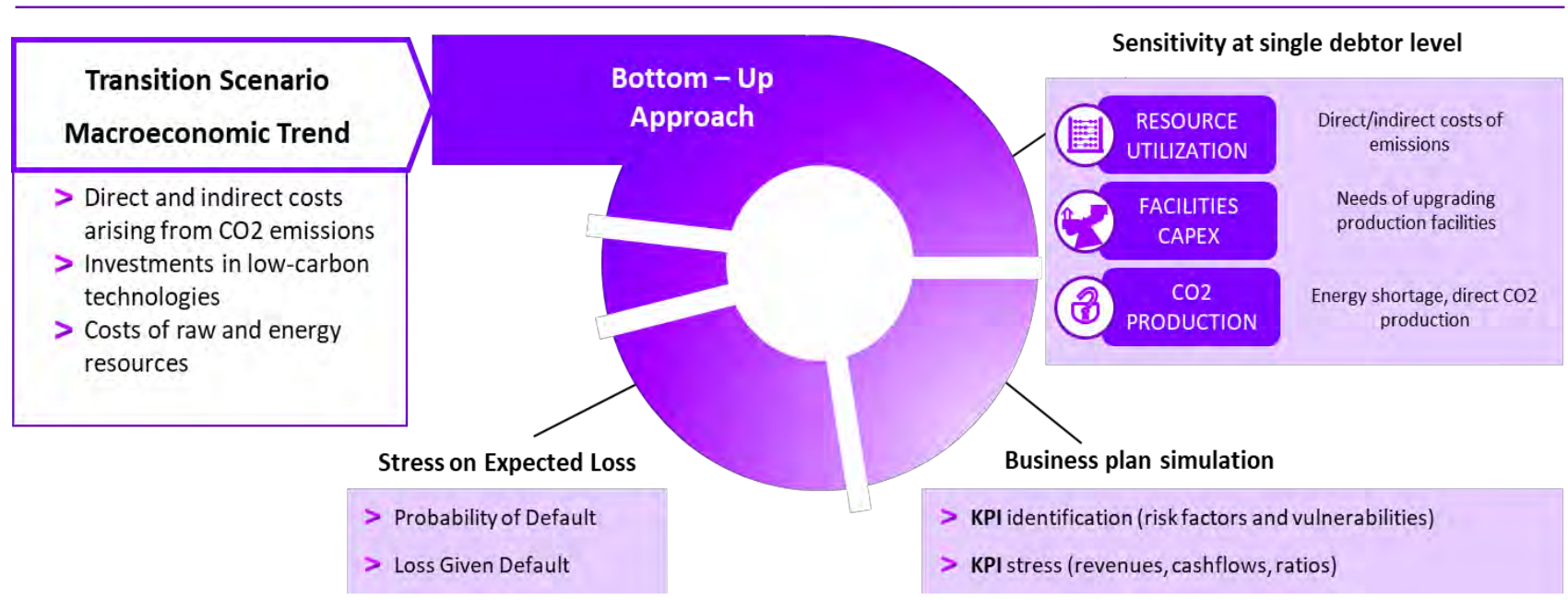

\subsection{Drill-down of the 4 transition risk scenarios: adaptation of the DNB approach}

Climate scenarios can generate projections in line with stress exercises by leveraging two key factors generally accepted in the literature: climate policies on $\mathrm{CO} 2$ emissions and technological developments in the energy sector, in particular on the renewable side (see for example CISL (2015) [9] and CERS/ESRB (2016) [8]).

Very interesting on the subject is the working paper proposed by the central bank of the Netherlands (DNB, 2019 [40]) which proposes 4 transition scenarios that leverage exogenous shocks deriving from the implementation of a carbon tax at global level and from the introduction of new technologies or a combination of both shocks.

Instead, the approach presented in this work, illustrated in the table below, starts from the IPCC scenarios, proposing their enrichment also with the help of the transmission models used by banks (e.g. the aforementioned GVAR).

Table 8 - Estimation of stressed macro variables based on IPCC and GVAR projections (example)

\begin{tabular}{|c|c|c|c|c|c|c|}
\hline & 2021 & 2022 & 2023 & 2024 & 2025 & Totale \\
\hline \multicolumn{7}{|c|}{ Rapid Transition (Scenario 1 RCP2.6, SSP1, SPA1) } \\
\hline Italy GDP & $-1.89 \%$ & $-1.35 \%$ & $-0.81 \%$ & $-0.27 \%$ & $0.27 \%$ & $-4.00 \%$ \\
\hline Euribor 3M & $-0.35 \%$ & $-0.25 \%$ & $-0.15 \%$ & $0.00 \%$ & $0.10 \%$ & $-0.13 \%$ \\
\hline Equity ITA (FTSEMIB) & $-9.36 \%$ & $-6.48 \%$ & $-1.12 \%$ & $0.96 \%$ & $2.36 \%$ & $-13.38 \%$ \\
\hline Btp / Bund 10Y (Spread) & $1.60 \%$ & $1.50 \%$ & $1.40 \%$ & $1.30 \%$ & $1.30 \%$ & $1.42 \%$ \\
\hline \multicolumn{7}{|c|}{ Two Degree (Scenario 2 RCP4.5, SSP2, SPA2) } \\
\hline Italy GDP & $-1.77 \%$ & $-1.29 \%$ & $-0.82 \%$ & $-0.35 \%$ & $0.12 \%$ & $-4.05 \%$ \\
\hline Euribor 3M & $-0.40 \%$ & $-0.30 \%$ & $-0.20 \%$ & $-0.05 \%$ & $0.05 \%$ & $-0.18 \%$ \\
\hline Equity ITA (FTSEMIB) & $-8.36 \%$ & $-4.05 \%$ & $-0.60 \%$ & $0.56 \%$ & $1.68 \%$ & $-10.63 \%$ \\
\hline Btp / Bund 10Y (Spread) & $1.70 \%$ & $1.60 \%$ & $1.50 \%$ & $1.30 \%$ & $1.40 \%$ & $1.50 \%$ \\
\hline \multicolumn{7}{|c|}{ Business as Intended (Scenario 3 RCP6.0, SSP3, SPA3) } \\
\hline Italy GDP & $-2.05 \%$ & $-1.63 \%$ & $-1.21 \%$ & $-0.79 \%$ & $-0.37 \%$ & $-5.91 \%$ \\
\hline Euribor 3M & $-0.40 \%$ & $-0.30 \%$ & $-0.30 \%$ & $-0.20 \%$ & $0.00 \%$ & $-0.24 \%$ \\
\hline Equity ITA (FTSEMIB) & $-12.60 \%$ & $-9.08 \%$ & $-3.96 \%$ & $2.16 \%$ & $3.36 \%$ & $-19.41 \%$ \\
\hline Btp / Bund 10Y (Spread) & $1.90 \%$ & $2.10 \%$ & $1.80 \%$ & $1.50 \%$ & $1.50 \%$ & $1.76 \%$ \\
\hline
\end{tabular}




\begin{tabular}{|c|c|c|c|c|c|c|c|}
\hline \multicolumn{7}{|c|}{ Business as Usual (Scenario 4 RCP8.5, SSP5) } \\
\hline Italy GDP & $-1.38 \%$ & $-0.81 \%$ & $-0.24 \%$ & $0.34 \%$ & $0.91 \%$ & $-1.19 \%$ \\
\hline Euribor 3M & $-0.30 \%$ & $-0.20 \%$ & $-0.10 \%$ & $0.05 \%$ & $0.15 \%$ & $-0.08 \%$ \\
\hline Equity ITA (FTSEMIB) & $-6.56 \%$ & $-4.44 \%$ & $-0.96 \%$ & $1.53 \%$ & $3.08 \%$ & $-7.45 \%$ \\
\hline Btp / Bund 10Y (Spread) & $1.50 \%$ & $1.40 \%$ & $1.50 \%$ & $1.50 \%$ & $1.60 \%$ & $1.50 \%$ \\
\hline
\end{tabular}

Given the lack of historical data, it is not immediately obvious how to choose the shocks necessary to complete the 4 transition scenarios. The underlying hypothesis concerns the impact that climate policies can have on oil price trends and consequently, through the implicit correlations in GVAR models, on other variables such as interest rates, equities and credit spreads between different countries. For calibration, the hypothetical introduction of a carbon tax of $\$ 100$ per ton of CO2 was converted into the

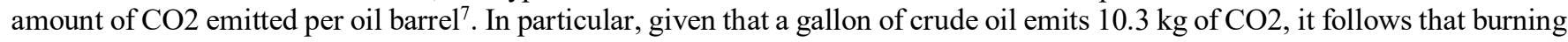
a barrel of oil, which contains 42 gallons, will emit $432 \mathrm{~kg}$ of $\mathrm{CO} 2$ in the event of a carbon tax $\$ 100$ per ton $(\$ 100 * 0.432=\$ 43.20)$.

The scenarios that incorporate a technological shock, mainly the first and second, but to a lesser extent also Scenario 3 RCP6.0, SSP3, SPA3 (Business as Intended), instead focus on a progressive technological substitution. More specifically, it is assumed that the percentage of non-renewables over the total energy produced will be lower, over a 5-year time horizon, respectively by $25 \%, 15 \%$ and $5 \%$ in the Rapid Transition, Two Degree and Business as Intended scenarios. These shocks are substantial, even though several studies predict that renewables will play an important role in energy production by $2030^{8}$.

The last relevant hypothesis is present only in the last scenario, namely Scenario 4 RCP8.5, SSP5 (Business as Usual), and concerns a possible confidence shock. In the case of GVAR, it is possible to implement shocks on consumption and investments through exogenous shocks on the GDP trend of all countries considered.

\subsection{Top-Down approach: general framework for the implementation of the stress test analysis}

It remains to discuss how to transform scenario narratives into impacts on the financial system. With this aim, it is necessary to apply a mix of different methodological approaches which, as we have already seen, can be classified into two macro-categories: top-down and bottom-up approaches.

As for the top-down approaches, firstly we need to calculate the impacts on the various sectors derived from the narratives described above and, subsequently, disaggregate these impacts referring to specific drivers, for example the CO2 emissions of each individual borrower. The figure below describes this process which, also in this case, adapts the DNB proposal to the Italian context.

Figure 2 - Transition risk measurement: top-down approach

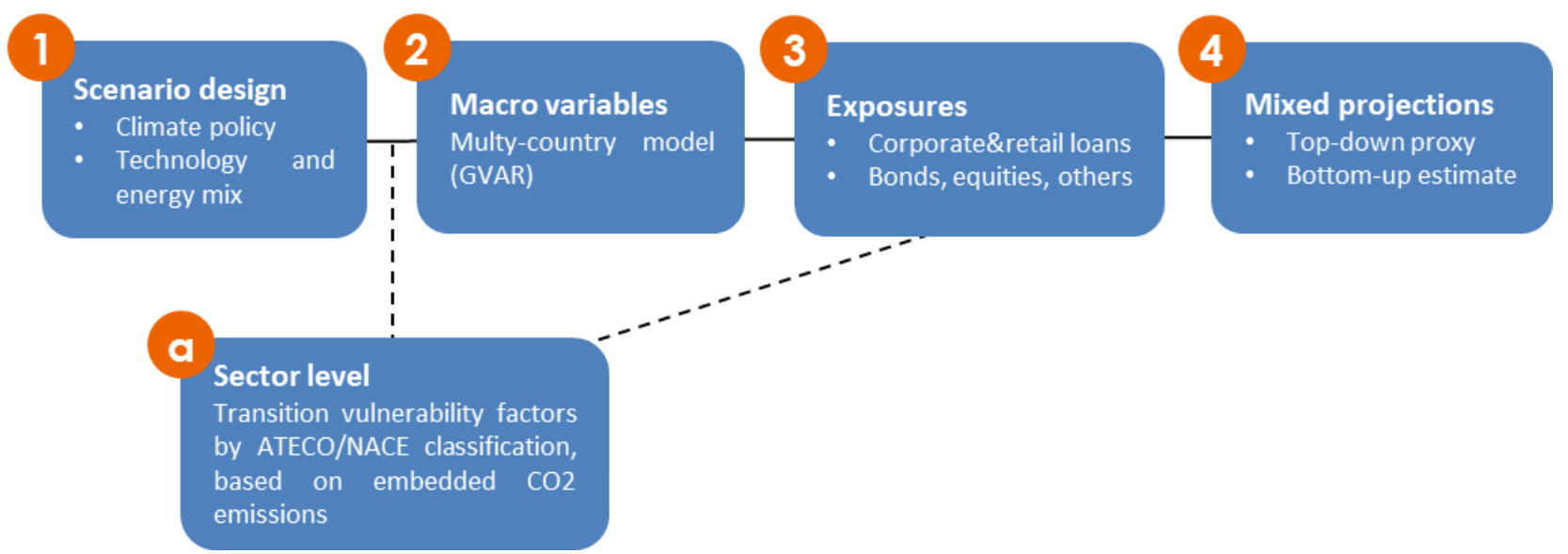

This figure is an elaboration from DNB 2018 [39]

We have already mentioned the points sub 1), sub 2) and sub 3), regarding how to generate reliable paths of the macroeconomic variables taking as a reference an econometric model. The next part of this type of approach, described below, involves the construction of specific factors to reallocate transition risk both across the different sectors and within them by using sectoral classifications available (ATECO/NACE).

\footnotetext{
${ }^{7}$ It is not difficult to find estimates even in the order of several hundred dollars per ton that will materialize within the next decade. For indepth discussions, see IPCC (2014) [22], Poelhekke (2017) [30] and Tol (2018) [33].

${ }^{8}$ See for example Creutzig et al. (2017) [12] or IEA (2017) [21].
} 


\subsection{Further hypotheses for the Top-Down approach: construction of the transition vulnerability factors and mapping by sectors}

Given that the transition to a low-carbon economy will affect companies that emit greater quantity of $\mathrm{CO} 2$ more than those that emit less, it is necessary to capture this heterogeneity both between sectors and, possibly, at the individual counterparty level. To obtain this result, it is possible to refer to the theory of the so-called transition vulnerability factors. These "drivers" vary according to the scenario in order to reflect the different types of risk present in the evolution path, thus making it possible to translate the general macroeconomic conditions into specific sector/segment losses.

With the aim to estimate the transition vulnerability factors of each sector/segment, the authors refer to the approach used in Hebbink et al. (2018). The input-output table of this study provides detailed information on suppliers and customers of each economic activity and the total $\mathrm{CO} 2$ of the production process. For implied emissions, it is instead possible to use information provided by different providers, generally available at the ATECO/NACE classification level.

In this way, the transition vulnerability factors can reflect the $\mathrm{CO} 2$ emissions implied in the value chain of the entire production process, with the consequence that a sector/segment containing twice $\mathrm{CO} 2$ emitted than the average will have to be affected two times higher. To give an example, in the automotive sector (sector NACE C29), the perspective of the value chain would lead to consider not only the obvious $\mathrm{CO} 2$ emissions of the car assembly, but also those related to the production of individual components, such as rubber to produce its tires (NACE sector C22). The following figure shows the drill-down of this example at the level of a single car produced.

Figure 3 - Example of $\mathrm{CO} 2$ emissions per single car produced

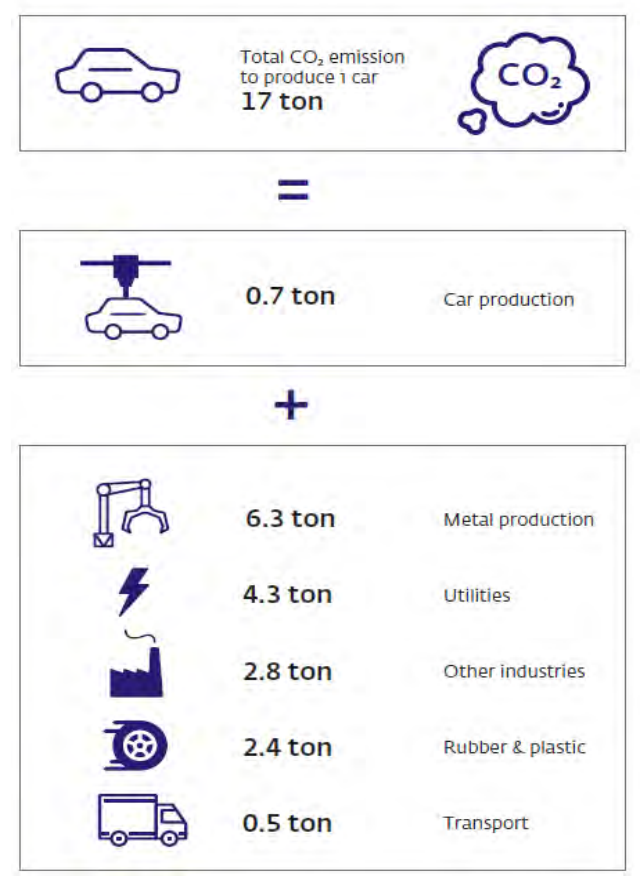

This figure is an elaboration from DNB 2018 [39]

\subsection{Bottom-Up approach: single name analysis and extension of the impacts at sectoral and portfolio level}

The bottom-up approach has a different logic than the previous one, as it analyzes the change in creditworthiness at the single borrower level and then extends the calibration to the portfolio, basing on a selection of names that represent the estimation sample. The bottom-up approach to transition risk (as described in UNEP FI / OW, 2018) can be divided into three phases:

- transition scenarios: description of economic developments by sectors and by geographical areas. The scenarios must provide a detailed narrative to define the exposure at the sector level;

- calibration at single name level: punctual assessment that tries to solve the lack of data using industry experts to estimate the impact of transition scenarios on individual borrowers;

- impact assessment on the portfolio: use of a systematic and repeatable approach to extend the risk to the rest of the portfolio (discussed in the next section).

As we have seen, in order to translate the scenario dynamics into impacts on the financial figures of the companies, the results must be summarized in a set of risk factors that insist on the main financial statement variables. Each sector/geographical area should therefore contain the main financial risk factors with respect to a baseline or a reference scenario, for example:

1. cost of direct emissions or increased costs of $\mathrm{CO2}$ emissions: in transition scenarios, the increase in costs is determined by the amount of emissions. In the real world, these costs could be translated into a carbon tax on greenhouse gas emitters;

2. cost of indirect emissions or increased costs of inputs: given that carbon-intensive inputs will be impacted on prices, sectors that use them most will be heavily penalized. Some costs may be passed on to customers through the product prices, indirectly balancing the cost increases; 
3. capital expenditure or increase in costs associated with investments to move to a low carbon economy: capital expenditure increases to meet the assumptions of an increase in energy demand and technology efficiency that are implicit in the scenarios;

4. changes in price and/or consumer demand: it is foreseeable that an increasing percentage of costs will be passed on to consumers. Consumers, in turn, will respond to rising prices by reducing the demand for certain goods and/or by increasing the demand for other products.

The joint assessment of these factors provides a significant overall picture for assessing the probability of default of the companies involved. At present, the scenario models only provide results at the sector level. Analysts could define specific sensitivities with the aim of specifying the impact of transition risk factors on a specific segment compared to the others. The following figure provides an exemplary and synthetic representation of the mapping process aimed at building sensitivities for the mining and metallurgical sector.

Figure 4 - Mapping for the sensitivity estimation of the mining and metallurgical sector
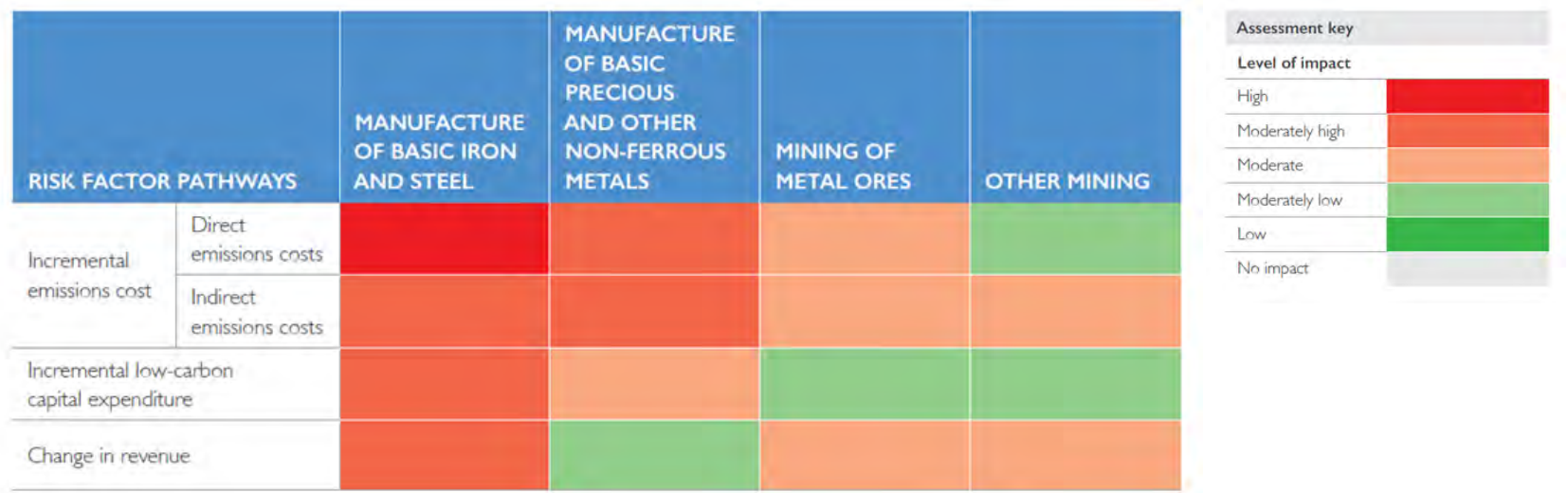

However, these sensitivities do not quantify the specific risk impact at the individual customer level. The calibration should be identified through an analysis at the level of the individual borrower. For the energy sector, for example, coal-fired power plants will have a greater sensitivity with a high negative impact to reduce the costs associated with emissions than a company already focused on nuclear or renewables. Another case could be the occurrence of increased sales for electric vehicle manufacturers even though car manufacturers as a whole could experience a sharp decline in demand.

The calibration at the single debtor level must therefore be based on the variables provided by the scenarios and then fill any information gaps using the judgment of internal analysts. Expert judgment could interpret each scenario and specify the potential impact on the creditworthiness of some particularly significant borrowers. Each of these "calibration points" will provide the information basis for extrapolating the impact to the rest of the portfolio.

Note that a bottom-up approach also allows for a bank-by-bank personalization. Through internal calibration, analysts have the opportunity to use the most appropriate tools for assessing the impact of the scenario, while ensuring that these decisions are consistent with their own risk appetite framework.

Lastly, calibration allows experts to reflect on how each individual company reacts to a transition scenario, basing on its operational characteristics. For example, an electric car manufacturer could be influenced differently than a traditional car manufacturer even in the presence of strong adaptability to market changes and competition. It goes without saying that such an in-depth analysis can only be limited to Most Significant Transactions (MST) and/or the most critical exposures.

\section{Transition risk: from projections to creditworthiness measurement}

Once the climatic costs have been estimated - ideally at the single counterparty level - the final step is to translate them into risk measures that can be introduced into the risk management systems. The risk measures traditionally used by financial intermediaries are the Probability of Default (PD), the Exposure at Default (EAD) and the Loss Given Default (LGD) and, as easily deductible from these measures, the credit rating process summarized by the concept of Expected Loss (EL). In summary:

$$
E L=P D \times E A D \times L G D
$$

\subsection{The impact on the Probability of Default (PD) of the Top-Down approach}

Since the introduction of the Basel 2 framework, banks have developed a significant amount of internal methodologies to assess the exposure of their portfolios to credit risk. The existing framework can be exploited, with the appropriate modifications, to evaluate the changes induced by the transition risk in the PDs.

In particular, we can refer to theories similar to those implicit in the Merton model to justify the impact on PDs of this new type of risk. As known, the model relates the PD with the probability that the future values of a company's assets may decrease below of its liabilities. Assuming that the other idiosyncratic and systemic risks remain unchanged, the change in PD could be measured by a shift from its initial value. This movement could be determined by the different risk factor paths of the scenarios, or by the top-down impacts on the various sectors calculated by using the aforementioned transition vulnerability factors. With regard to the top-down approach, the authors propose to use the equation set out below:

$$
P D_{i} \mid \mathrm{s}^{*}=\Phi\left(\Phi^{-1}\left(P D_{i, T T C}\right)-\frac{1}{\Gamma_{k}} \tau_{j, k}^{r}\left(\Phi^{-1}\left(P D_{0, T T C}^{s}\right)-\Phi^{-1}\left(P D_{t, T T C}^{S}\right)\right)\right)
$$




$$
\begin{gathered}
P D_{i} \mid \mathrm{s}^{*}=\text { scenario-adjusted PD of borrower } i \text { given sector/segment } s \\
P D_{i, T T C}=\text { through-the-cycle PD of borrower } i \\
\tau_{j, k}^{r}=\text { transition vulnerability factor of shock } r \text { in segment } j \text { of sector } k \\
\Gamma_{k}=\text { total sum of transition vulnerability factor within the sector } k \\
P D_{0, T T C}^{s}=\text { initial through-the-cycle PD of sector } s \\
P D_{t, T T C}^{s}=\text { through-the-cycle PD resulting from top-down stress for sector } s \\
\Phi=\text { standard normal cumulative distribution function }
\end{gathered}
$$

Essentially, this equation increases the initial PD based on a top-down estimated risk value through the scenarios' paths enriched with the GVAR model previously described. This movement is nothing more than the product between the outputs of the stress scenarios calculated at sector level and the sensitivity of the sector/segment, in this case represented by the transition vulnerability factor.

It also should be noted that the term $\Gamma_{k}$ is strictly necessary, since it allows to obtain that the sum of the weights concerning the implied $\mathrm{CO} 2$ per sector is always equal to 1 .

This methodology refers to the commonly used stress analyzes, adding new internally estimated parameters that take into account $\mathrm{CO} 2$ emissions. The framework can therefore be applied to all sectors and can take into consideration both the composition of each segment, the different transition vulnerability factors and, eventually, a judgmental override provided by credit analysts.

\subsection{The calibration of the PDs in the Bottom-Up approach}

As in the top-down approach, also for the bottom-up approach it is useful to refer to the theories implicit in the Merton model to calculate the impact of transition risk. For the bottom-up approach, the starting point in this case will not be the variation of the PDs expressed at sector level, but the calibration will start from the variation of the probability of default calculated for a specifically selected sample of borrowers.

The equation that was used is very similar to the one above and can be summarized as follows:

$$
P D_{i} \mid \mathrm{c}^{*}=\Phi\left(\Phi^{-1}\left(P D_{i, T T C}\right)-\frac{1}{\alpha_{k}} \sum_{j}\left(s_{j, k}^{r} f_{k}^{r}\right)\right)^{-1}
$$

$P D_{i} \mid \mathrm{c}^{*}=$ scenario-adjusted PD of borrower $i$

$P D_{i, T T C}=$ through-the-cycle PD of borrower $i$

$s_{j, k}^{r}=$ sensitivity of risk factor $r$ for segment/geographic area $j$ of sector $k$

$f_{k}^{r}=$ evolutionary path for risk factor $r$ of sector $k$

$\alpha_{k}=$ calibration factor of sector $k$

$$
\frac{1}{\alpha_{k}} \sum_{j}\left(s_{j, k}^{r} f_{k}^{r}\right)=\text { Climate Credit Quality Index (CCQI) for segment/geographic area } j
$$

This equation shifts the PD from its normal time path, based on a value that is defined as the "climate credit quality index" (UNEP FI / OW, 2018 [34]). This index is nothing more than the sum of the products between the outputs of the stress scenarios, the so-called risk factors, and the sensitivity of the sector (high/medium/low), multiplied by a specific calibration factor $\alpha_{k}$ of the reference sector. From a theoretical perspective, CCQI identifies the size of the sectoral impact of the scenario, normalizing the variation of risk factors so that they can be interpreted with a unitary distribution, as required by the Merton framework.

It should be noted that this type of methodology interprets the qualitative levels of sensitivity provided by credit analysts (expert judgment) as optimization constraints. These constraints ensure that the sensitivity values, resulting from the calibration, are consistent with the expert-based assessments (e.g. "high" sensitivities, associated with a target beta of 1.2, will have a more negative impact than those marked as "low", corresponding to a target beta of 0.8 ).

Once all parameters of the above equations have been calibrated, it is possible to estimate the PD implied in the different stress scenarios for all debtors of a given segment. It should be remembered that also in this case the methodology refers to the most commonly used stress techniques, but consistently with internally estimated parameters and allowing for any customization. The framework can therefore be applied to all sectors and can take into account both the composition of each segment, the series of sensitivities $s_{j, k}^{r}$ and a different judgmental calibration provided by internal analysts.

\subsection{The effects on Loss Given Default (LGD) of the transition risk}

The estimation of LGD variations, the second element of the expected loss, should be largely guided by the type and value of collaterals provided at the individual transaction level. Banks should identify cases where an industry-specific LGD assessment is sufficient and where customized valuations should be developed. Alternatively, simplified approaches could be used, for example by assuming that the impact on LGD is based on its relationship to PD. More in depth:

- directly evaluation of LGD based on stressed PD using the Frye-Jacobs formula, which provides a single generic relationship parameter between PD and LGD;

$$
L G D_{\text {Transition }}=\frac{\Phi\left(\Phi^{-1}\left(P D_{\text {Transition }}\right)-\left[\Phi^{-1}\left(P D_{T T C}\right)-\Phi^{-1}\left(P D_{T T C} L G D\right)\right]\right)}{P D_{\text {Transition }}}
$$




$$
\begin{aligned}
& L G D_{\text {Transition }}=\text { scenario-adjusted LGD } \\
& P D_{\text {Transition }}=\text { scenario-adjusted PD } \\
& P D_{T T C}=\text { initial PD, portfolio specific } \\
& L G D=\text { implied starting LGD }
\end{aligned}
$$

- $\quad$ use of LGD forecasts based on an internally estimated correlation between PD and LGD.

Given the lack of data and a shared approach, it is preferable to adopt the Frye-Jacobs relationship as a reasonable approximation. Obviously, all the methodologies described in the previous sections represent a first starting point for any future insights into the transition risk.

\section{Physical risk: the assessment of impacts on creditworthiness}

In this section, we propose a framework for assessing the impact of physical risk on creditworthiness. The methodology addresses, both in top-down and bottom-up modalities, the estimate of the reduced ability of a borrower to meet payment commitments following the occurrence of an event linked to climate change. The assessment of the change in credit risk takes the form of a revision of both the estimate of the probability of default and the LGD (due to the direct impact of physical events on the value of the borrower's assets) with different levels of granularity in the bottom-up and top-down:

Table 9 - Physical risk: top-down and bottom-up approaches

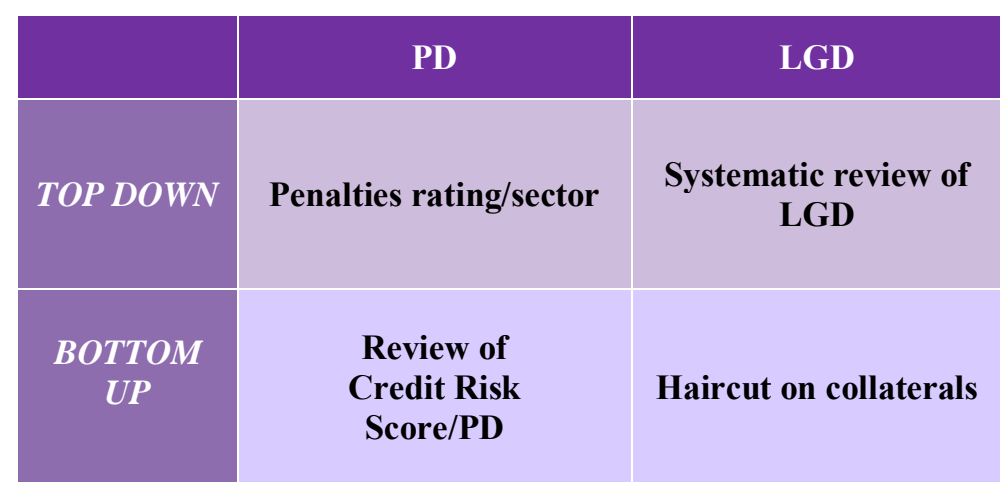

The ability of a single institution to apply the framework depends on the robustness of the database linked to the borrowers' assessment: therefore, particular attention must be paid to the structure of the information to be used for a complete evaluation of the risk deriving from the climate change.

\subsection{The frameworks of the Bank of England and ClimateWise}

For the estimation of the impact of physical risk, the literature essentially provides two overall frameworks, mainly related to the anglo-saxon and reinsurance markets. The main proposals are those of the Bank of England 2018 [3] and 2019 [4] ${ }^{9} 10$ and ClimateWise [11 $]^{11}$, the latter resulting from an insurance association whose secretariat is held by the University of Cambridge. Both frameworks are mainly oriented to the insurance sector.

The approach proposed by the Bank of England is essentially divided into six phases:

Figure 5 - Bank of England framework for the physical risk

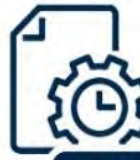

Stage 1:

Identify

business

decision(s)
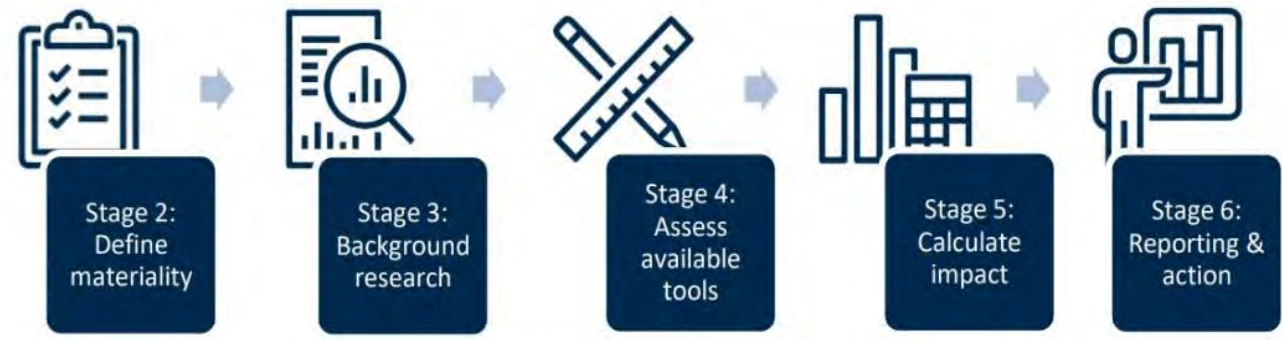

Figure taken from Bank of England, 2019 [4]

\footnotetext{
${ }^{9}$ Bank of England. (2018). Transition in thinking: The impact of climatechange on the UK banking sector.

${ }^{10}$ Bank of England (2019). A framework for assessing financial impacts of physical climate change. A practitioner's aide for the general insurance sector.

${ }^{11}$ www.climatewise.org.
} 
In describing its specific implementation, the Bank of England makes particularly interesting considerations regarding to:

- the assessment of the materiality of the physical risk, suggesting that the estimations should be based on the scientific evidence that links climate change to the modification of a specific phenomenon. In particular, if the impact is immaterial, a threshold should be defined beyond which the risk can become significant;

- the choice of scenarios makes explicit reference to the RCP previously described;

- the pros and cons of the different tools available (expert judgment, hazard maps, footprints and catastrophe models), suggesting to adapt the tools to the needs of the assessment (for example, the use of event footprints and catastrophe models is recommended for portfolio analysis, as well as hazard maps at the level of individual exposures and, in general, an accurate recalibration of tools based on observations and future scenarios is recommended);

- the accurate representation of the uncertainty of the estimates (based on sensitivities and qualitative assumptions).

The Bank of England approach is essentially based on the same considerations that insurance companies normally include in the ORSA reports: in particular, the choices for the development of new products or business lines in relation to their internal risk appetites.

Instead, the ClimateWise proposal is articulated in a four steps approach (ClimateWise [10]);

1. collection of data on exposures: in particular the geographical location is essential since most climate risk models require data with a high spatial resolution;

2. selection of the model for natural disasters: the approach lists several modeling options. The OASIS project deserves particular mention ${ }^{12}$, given that is an open source model;

3. selection of the climate change scenario: the definition of the scenario normally requires managing the discrepancy between the geographical resolution of the catastrophe models and the scenarios, usually defined with a lower precision;

4. execution of the catastrophe model: although catastrophe models are very different, they provide some standard outputs:

- the AAL, (Average Annual Loss), loss in the value of portfolio assets;

- annual probability of occurrence;

- return time;

- Annual Exedance Curve: probability of exceeding certain loss levels.

The fundamental requirements of the ClimateWise approach are the classifications of exposures, which should be geolocated and equipped with attributes that describe the exposure to risk factors, the use of catastrophe models for the deduction of statistics and the use of models for the reactivity of the prices.

\subsection{Relationship between physical risk and creditworthiness}

The proposed approach to estimate the exposure to physical risk related to credit exposures will be divided into both for the topdown and bottom-up approach in the following steps:

- define a series of climate change scenarios (see for example AIFIRM, [1]);

- $\quad$ explain, using an econometric model, the effects of physical risk on certain business sectors and geographical areas over a given time horizon (by using sensitivities and shock factors);

- $\quad$ apply, for each of these scenarios, the results of the previous point, evaluating the changes in PD and LGD of credit positions;

- $\quad$ organize, based on the results of the previous point, a specific reporting that highlights the changes in economic capital and average defaults.

This articulation is in particular consistent with the Bank of England approach described above, which aims to define:

- the scope of application of physical risk, which in the case of this study is limited to the assessment of credit risk;

- the materiality aspects, to be considered on the basis of existing portfolios;

- the need to consider the "background research", which led to the decision to adhere to the research available at the drafting date of this study;

- the choice of tools: mainly the available risk maps will be used. The in-depth analysis of catastrophe models goes beyond the scope of this analysis;

- calculation of impacts, based on sensitivity techniques, as clarified in the following paragraphs;

- reporting, which in the case of this study will essentially focus on the estimate of the worsening of creditworthiness and the consequent increased need for economic capital.

\footnotetext{
$12 \mathrm{https}: / /$ climateoasis.com.
} 
In the Italian banking sector, the use of "reduced form" models or credit risk scores is widespread, especially for the evaluation of credit lines. A credit risk score is typically the result of a linear discriminant analysis, which assigns weights to a certain group of observed variables (for example in the case of the Z-score, Altman [2], a series of financial ratios).

The weighted sum of the variables determines the score: a discriminating threshold is then defined in relation to which an exposure is classified as a probable default.

In practice it is not only necessary to link the credit risk score to an insolvency forecast, but also to an assessment of the probability of default: this happens empirically (observing the default rates) or analytically (with a transfer function that links the score to the PD, typically a logistics):

$$
P D=f(Z)=\frac{1}{1+\exp [a+b Z]}
$$

\subsection{Proposals for implementing the Top-Down approach}

In a top-down approach, the assessment of physical risk can be defined in a similar way to what has already been seen for transition risk, bearing in mind that, if the transition risk by its nature allows a sectoral declination, the physical risk, depending on from micro-local exposure profiles (e.g. geographical or idiosyncratic such as the layout of production facilities), is better described with a bottom-up approach ${ }^{13}$. As with transition risk, physical risk assessment can also be carried out by introducing corrections to the probability of default on a geo-sectorial basis. In particular, having defined $\pi_{0 P}$ and $\pi_{0 Q}$ the neutral and current probability of default observed without taking into account the risk of climate change, we can introduce the following corrective (which can be interpreted as a credit quality factor for physical risk):

$$
q_{j, k}=\frac{1}{\alpha_{j}} \sum_{r} s_{j}^{r} \times f_{k}^{r}
$$

where index $\mathrm{k}$ represents the climate change scenario considered, index $\mathrm{j}$ the geo-sectorial declination and index $\mathrm{k}$ the declination by vulnerability factor, while the letter $\alpha$ indicates a calibration factor, the letter $\mathrm{s}$ a factor of sensitivity and the letter $\mathrm{f}$ the shock of the factor $\mathrm{r}$ determining the PD (the factor $\mathrm{f}$ is valued by developing the quantitative description of the scenarios). With respect to the transition risk approach (in which $\mathrm{CO} 2$ emissions are used as the main driver), in this case the sensitivity factors will have to take into account the direct effects on the borrower production capacity and on the costs that it should bear both to cope with changes in the production chain and with any extreme events (and can be calculated simulating the effects of incremental physical risk on average balance sheet setup for a particular class of borrowers). Therefore, the calibration of the various scenarios should take into account both the forecast assessments and the historical response to extreme events in the variation of the probability of default (extreme events can be taken into account through the calibration factor).

The (current) default probability $\pi_{k Q}$ in the climate change scenario $\mathrm{k}$ is obtained as:

$$
\pi_{k Q}=\Phi\left(\Phi^{-1}\left(\pi_{0 Q}\right)-q_{j, k}\right)=\Phi\left(\Phi^{-1}\left(\pi_{0 P}\right)+\frac{\mu-r}{\sigma} \sqrt{ } \tau-q_{j, k}\right)
$$

where the term $\frac{\mu-r}{\sigma} \sqrt{ } \tau$ represents the market price of risk (see for example Crouhy et al. 2000 [13]).

The corrective introduced to take into account the physical risk can be seen as a change in the market price of risk declined according to the geo-sectorial key adopted for the definition of the sensitivity: the underlying hypothesis, assuming risk parity, is a reduction in the value of the return on equity, due to the manifestation of physical risk in an idiosyncratic form. It should be noted that this correction only impacts the current probability, since by construction the risk-neutral probability already contains all the market evaluations also with respect to physical risk factors ${ }^{14}$.

From the point of view of the change in LGD in a top-down approach, the value of the collateral is not explicitly taken into account, but it is possible to proceed, as already seen, to increase the LGD starting from the PD (following the approach proposed by J. Frye, M. Jacobs Jr., 2012, [19], the expected variation of LGD can be regarded as a function of the variation of PD).

\subsection{Bottom-Up approach: first developments}

The bottom-up framework focuses on the assessment of the physical risk for each borrower, taking into account the geo-sectorial and idiosyncratic characteristics. Also in this case, the creditworthiness of the various exposures will be linked to the changes in the fundamental quantities present in the expected loss: probability of default and LGD.

Relating to the probability of default, the PD will be assumed dependent from a linear credit risk score $\mathrm{Z}$ as defined below:

$$
P D=f(Z) ; Z=\sum_{j=1 \ldots N_{j}} \beta_{j} w_{j}
$$

where the weights $w_{j}$ are considered assigned, while the variables $\beta_{j}$ vary according to the borrower.

13 Regard this aspects, it is useful to recall the comparison with the Solvency II insurance legislation (Commission delegated regulation 35/2015, articles $120-126$ ), which in fact $\mathrm{s}_{\mathrm{uggest}} \mathrm{s}^{\mathrm{s}}$ a bottom_up approach.

14 This statement does not take into account the fact that the risk neutral probability is related to the weighted realization with the neutral probability of the different scenarios intended not only as RCP, but also as SSP/SPA. Therefore, the risk neutral changes in probability, dependant on the realization of a single scenario, should also be considered. 
Concerning the LGD, the impact is evaluated in both simplified and advanced approaches considering collateralisation: the physical risk related to this element is relevant, since on the one hand it must be considered that a physical climatic event damages significantly the physical assets placed as collateral for a loan and, on the other hand, the demand for conservativeness in estimating LGDs in advanced approaches requires that extreme events also be taken into account. The relationship could be:

$$
L G D_{i}=L G D_{\text {uncoll }} \times \frac{E_{i}-\text { Coll }}{E_{i}}
$$

The effect of physical risk has basically two types of effects on the borrower creditworthiness: an incremental effect, due to the progressive change of the environment in which the borrower conducts its activities, and a catastrophic effect, due to the occurrence of an extreme event which directly affects the productive assets of the borrower or its supply and sale chains.

To assess the impact of climate change risk, physical risk side, the authors propose an approach consistent with the indications of Acclimatise (UNEP FI [36]):

- description of the borrower operating environment (regional, national, global operations, etc.) and selection of k climate change scenarios defined on a geographic basis;

- for each scenario $\mathrm{k}$, identification of the economic aggregates $P_{i}^{k}$ relating to the borrower (affecting the variables used in the $\mathrm{Z}$ score, or the evaluation of collateral) that could undergo variations as a result of physical risk (for example, variation in the plants' capacity, variation in sales prices, variation in purchase prices, etc.);

- $\quad$ estimate of the variations $\Delta_{i n c r} P_{i}^{k}=\left(P_{i}^{k}-P_{i}^{0}\right) / P_{i}^{0}$ that would occur in the various scenarios due to the changed operating conditions of the borrower;

- $\quad$ for each type of extreme event q, calculation of the increase in probability $\Delta \psi_{q}^{k}$ that affects the borrower in scenario k. The increase is calculated as the difference between the probability of the event and the probability found in the current observations $\Delta \psi_{q}^{k}=\psi_{q}^{k}-\psi_{q}^{0}$. The shocks that the occurrence of a single event would happen are then assessed $\Delta_{\text {cat }} P_{i}^{q}=\left(P_{i}^{q}-P_{i}^{0}\right) / P_{i}^{0}$

- determination of which aggregate $\beta_{j}$, used by the credit risk score, is influenced by assigning specific coefficients $\delta_{j}^{i}=$ $\frac{\partial \beta_{j}}{\partial P_{i}}$

- $\quad$ evaluation of the Z-score variation by combining the incremental and catastrophic effects:

$$
\Delta Z^{k}=\sum_{j} \alpha_{j} \Delta \beta_{j}=\sum_{j} \alpha_{j}\left(\sum_{i} \delta_{j}^{i}\left(\Delta_{\text {incr }} P_{i}^{k}+\sum_{q} \Delta \psi_{q}^{k} \Delta_{c a t} P_{i}^{q}\right) P_{i}^{0}\right)
$$

- $\quad$ final estimate of the PD variation by deriving the transfer function from Z-Score to PD:

$$
\Delta P D^{k}=f^{\prime}(Z) \Delta Z^{k}
$$

With regard to collateral, identified with $A$ the assets placed as collateral, the changes in the value of the assets are directly estimated according to a similar scheme $\left(\Delta_{\text {incr }} A_{i}^{k}\right.$ and $\left.\Delta_{c a t} A_{i}^{q}\right)$, and the consequent variation $\Delta \operatorname{Coll}^{k}$ is thus determined:

$$
\Delta L G D^{k}=-L G D_{\text {uncoll }} \times\left(\frac{\Delta \text { Coll }^{k}}{E_{i}}\right)
$$

Alternatively, where there is no precise information on collaterals (and on the effect of climate change on them), the same approach of the previous paragraphs can be used (i.e. applying the Frye Jacobs formula).

Clearly, for the application of the framework several factors are fundamental:

- an accurate database that allows the identification of the individual production units of the borrower, with reference to both their geographical location, their physical characteristics (type of buildings, security systems, etc.) and the production processes (type of supply chain, etc.);

- a clear definition of the climatic scenarios that allows, from a qualitative point of view, to carry out an accurate inventory of both the incremental effects and the possible catastrophic events;

- the quantitative specification of the factors $\Delta_{\text {incr }} P_{i}^{k}, \Delta_{\text {cat }} P_{i}^{q}, \Delta_{\text {incr }} A_{i}^{k}, \Delta_{\text {cat }} A_{i}^{q}$, for which it is possible to use both sources available in the literature, econometric evaluations (e.g. global econometric models ${ }^{15}$ ) and expert judgment, applying methods consistent with the aforementioned framework of the Bank of England;

- the quantitative specification of the factors $\Delta \psi_{q}^{k}$, for which we can refer both to officially recognized sources, for example the UNEP Global Risk Data Platform which has risk maps available at national level (e.g. the maps drawn up by the various regional environmental agencies pursuant to Directive 2007/ 60/EC for the risk of floods).

\section{Conclusions and final remarks}

This paper is inspired by the strong acceleration given to the financial industry by the TEG work on the EU taxonomy, without mentioning the recent ECB Guide on climate and environmental risks (ECB, 2020), and aims to be a useful tool for a possible 
implementation of these issues in internal processes of the financial intermediaries. Without claiming to be exhaustive, the authors provided an overview of the available literature on physical and transition risk, focusing on forward looking scenario analysis, and in-depth examinations of quantitative and qualitative tools commonly considered fundamental in the assessment of this type of risk.

However, several issues remain open and have been addressed in the discussion in a qualitative way and within the limits of reasonableness: 1) address the lack of historical data, 2) choice of the correct time horizon for the risk models used, 3) find the right level of data granularity , 4) identification of relevant KPIs and KRIs to be used for climate risk exposures and 5) translation of economic impact into financial risk metrics.

The proposed methodologies should therefore be seen as a first attempt to assess the potential impact of climate change scenarios on the capital and liquidity of banks, to be refined with the development of new approaches and enrichment of data that in the natural evolutionary path will gradually become available.

\section{Giuliana Birindelli, Vera Palea, Luca Trussoni, Fabio Verachi}

\section{Bibliography}

[1] AIFIRM, Climate Change: assess and advance awareness of a new Financial Risk, Position Paper n. 20, April 2020

[2] Altman, E. I. "A fifty-year retrospective on credit risk models, the Altman Z-score family of models and their applications to financial markets and managerial strategies." Journal of Credit Risk 14 (2018): 1-34

[3] Bank of England, Transition in thinking: The impact of climatechange on the UK banking sector, September 2018

[4] Bank of England, A framework for assessing financial impacts of physical climate change. A practitioner's aide for the general insurance sector, May 2019

[5] M. Barbanti Brodano, F. Cocco, G. Moramarco, Global Economy, Sovereign Spreads and Public Debt in the Euro Area: a GVAR Approach, Prometeia Technical Report, 2014

[6] S. Battiston, A. Mandel,F. Schuetze,G. Visentin (2017). A Climate Stress-Test of the Financial System. Nature Climate Change. 7. $10.2139 /$ ssrn.2726076

[7] Giusy Capasso, Gianfranco Gianfrate, Marco Spinelli, Climate change and credit risk, Journal of Cleaner Production, Volume 266, 2020, 121634, ISSN 0959-6526, https://doi.org/10.1016/j.jclepro.2020.121634

[8] CERS/ESRB, European Systemic Risk Board (2016). Too late, too sudden: Transition to a low-carbon economy and systemic risk, Reports of the Advisory Scientific Committee. No 6 / February 2016

[9] Cambridge Institute for Sustainability Leadership (CISL), (2015, November). Unhedgeable risk: How climate change sentiment impacts investment. Cambridge, UK: Cambridge Institute for Sustainability Leadership

[10] Cambridge Institute for Sustainability Leadership (CISL), (2019, February). Physical risk framework: Understanding the impacts of climate change on real estate lending and investment portfolios. Cambridge, UK: Cambridge Institute for Sustainability Leadership

[11] www.climatewise.org

[12] Creutzig, F., Agoston, P., Goldschmidt, J. et al. The underestimated potential of solar energy to mitigate climate change. Nat Energy 2, 17140 (2017). https://doi.org/10.1038/nenergy.2017.140

[13] M. Crouhy, D. Galai, R. Mark, Risk Management, New York, McGraw Hill 2000

[14] Dietz, Simon \& Bowen, Alex \& Dixon, Charlie \& Gradwell, Philip. (2016). 'Climate value at risk' of global financial assets. Nature Climate Change. 6. 10.1038/nclimate2972

[15] F. di Mauro, H. Pesaran, The GVAR Handbook: Structure and Applications of a Macro Model of the Global Economy for Policy Analysis, Oxford University Press, 2013

[16] ECB, Guide on climate-related and environmental risks - Supervisory expectations relating to risk management and disclosure, May 2020

[17] ENI scuola energia e ambiente, Scenari futuri secondo l'IPCC, http://www.eniscuola.net/argomento/cambiamenti-climatici/scenarifuturi/scenari-futuri-secondo-lipce/

[18] I. Faiella, F. Natoli, Climate change and bank lending: the case of flood risk in Italy, Conference on Climate Change and its Impact in the Financial System Mexico City, 5-6 December 2019

[19] J. Frye, M. Jacobs Jr., Credit loss and systematic loss given default, The Journal of Credit Risk Vol 8, 1-32, Spring 2012

[20] IPCC, Special Reports on Emission Scenarios, Cambridge University Press 2000

[21] IEA, World Energy Report 2017, a world in trasformation, Flagship report - November 2017

[22] IPCC (2014) Climate Change 2014 Mitigation of Climate Change. Working Group 3 Contribution to the Fifth Assessment Report of the Intergovernmental Panel on Climate Change (IPCC). Technical Summary and Chapter 6 (Assessing Transformation Pathways)

[23] Jansen D.J. (2019), A Framework for Measuring Financial Stress under Disruptive Energy Transition Scenarios, EBA/EBF Workshop on Sustainable Finance, 4 April

[24] K. Riahi, D. P. van Vuuren, E. Kriegler, J. Edmonds, B. C. O’Neill, S. Fujimori, N. Bauer, K. Calvin, R. Dellink, O. Fricko, W. Lutz, A. Popp, J. Crespo Cuaresma, Samir KC, M. Leimbach, L. Jiang, T. Kram, S. Rao, J. Emmerling, K. Ebi, T. Hasegawa, P. Havlik, F. Humpenöder, L. Aleluia Da Silva, S. Smith, E. Stehfest, V. Bosetti, J. Eom, D. Gernaat, T. Masui, J. Rogelj, J. Strefler, L. Drouet, V. Krey, G. Luderer, M. Harmsen, K. Takahashi, L. Baumstark, J. C. Doelman, M. Kainuma, Z. Klimont, G. Marangoni, H. Lotze-Campen, M. Obersteiner, A. Tabeau, M. Tavoni, The Shared Socioeconomic Pathways and their energy, land use, and greenhouse gas emissions implications: An overview, Global Environmental Change, Volume 42, 2017, Pages 153-168, ISSN 0959-3780, https://doi.org/10.1016/j.gloenvcha.2016.05.009

[25] Monnin, P. (2018). Integrating Climate Risks into Credit Risk Assessment-Current Methodologies and the Case of Central Banks Corporate Bond Purchases. Council on Economic Policies, Discussion Note, 4

[26] OECD Guidelines on Multinational Enterprises and for Responsible Business Conduct, https://www.oecd.org/corporate/mne/responsiblebusiness-conduct-matters.htm 
[27] O'Neill, B.C., Kriegler, E., Riahi, K., et al., 2014. A new scenario framework for climate change research: the concept of shared socioeconomic pathways. Clim. Chang. 122, 387-400

[28] Brian C. O’Neill, Elmar Kriegler, Kristie L. Ebi, Eric Kemp-Benedict, Keywan Riahi, Dale S. Rothman, Bas J. van Ruijven, Detlef P. van Vuuren, Joern Birkmann, Kasper Kok, Marc Levy, William Solecki, The roads ahead: Narratives for shared socioeconomic pathways describing world futures in the 21st century, Global Environmental Change, Volume 42, 2017, Pages 169-180, ISSN 0959-3780, https://doi.org/10.1016/j.gloenvcha.2015.01.004

[29] See [34] and [35]

[30] Steven Poelhekke, How expensive should CO2 be? Fuel for the debate on optimal climate policy, DNB Working Paper n. 579 (2017)

[31] Pesaran M.H., T. Schuermann, and S.M. Weiner, Modelling Regional Interdependencies using a Global Error Correcting Macroeconometric Model, Journal of Business and Economic Statistics, 2004, 22, 129-162

[32] Technical Expert Group on sustainable finance (TEG), TEG Final Report on the EU taxonomy, European Commission, March 2020. The report published in March 2020 revise and update, also in light of the evidence gathered in the public consultation phase, the first version of the documents published in June 2019

[33] Richard S J Tol, The Economic Impacts of Climate Change, Review of Environmental Economics and Policy, Volume 12, Issue 1, Winter 2018, Pages 4-25, https://doi.org/10.1093/reep/rex027

[34] UNEP FI, Extending Our Horizons: Assessing Credit Risk and Opportunity in a Changing Climate - PART1: Transition-related risks \& opportunities, April 2018

[35] UNEP FI, Navigating a New Climate: Assessing Credit Risk and Opportunity in a Changing Climate - PART2: Physical-related risks and opportunities, July 2018

[36] UNEP FI, Piloting the TCFD recommendation for Banks, July-August 2019

[37] UN Guiding Principles on Business and Human Rights, International Labor Organizations, https://www.ungpreporting.org/res ources/theungps/

[38] van Vuuren, D.P., Edmonds, J., Kainuma, M. et al. The representative concentration pathways: an overview. Climatic Change 109, 5 (2011). https://doi.org/10.1007/s10584-011-0148-z

[39] R. Vermeulen, E. Schets, M. Lohuis, B. Kölbl, D.J. Jansen, W. Heeringa, An energy transition risk stress test for the financial system of the Netherlands, DNB Occasional study n. 7, 2018

[40] R. Vermeulen, E. Schets, M. Lohuis, B. Kölbl, D.J. Jansen, W. Heeringa, The Heat is on: a framework for measuring financial stress under disruptive energy transition scenarios, DNB Working Papers 625, Netherlands Central Bank, Research Department, 2019 


\section{Critical analysis of the most widespread methodologies for the simulation of the short rate dynamics under extreme market conditions}

Pier Giuseppe Giribone (University of Genoa, Banca Carige)

Article submitted to double-blind peer review, received on 4th November 2020 and accepted on 28th November 2020

\section{Abstract}

This study proposes an analysis of the main drawbacks emerged when adopting the traditional short rate dynamics under extreme market conditions such as under negative interest rates.

In fact, this condition has led to invalidate the use of the majority of the most popular stochastic differential equations (SDE) reported in the scientific literature.

The first part of the paper makes an overall introduction to the problem, analyzing it from different perspectives. Given that the author dealt with these topics in previous research items, the objective of the paper is to focus on only one of these aspects which was not scrutinized before and to examine it in full detail.

As a result, the most popular stochastic dynamics are shown in the second part and the problems raised by their numerical integration are then discussed.

Starting from the equations for which it is feasible to implement a numerical scheme for their solution, the problem thus becomes how to find a reasonable estimation for the SDE parameters. The third section deals with the problems occurred in the application of Maximum Likelihood Estimation (MLE) caused by the negative interest rates during the implementation of the wellestablished approaches. For every analyzed dynamics a real market case is provided.

The paper highlights the appropriateness of the Hull-White model which can be considered a feasible and reliable solution for simulating short rates also under extreme market conditions.

Key Words: negative interest rates, short rate models, short rate dynamics calibration, stochastic differential equation, interest rates simulation

\section{Introduction}

The interest in the analysis and discussion of the problems related to the advent of negative interest rates (present for more than four years in the Eurozone) was rekindled when the main info-providers officially announced that they would adopt the same conventions in estimating the fair value and risk measures even for deals denominated in the USD currency.

Editors of the most important worldwide newspapers, including the Financial Times, took an interest in the consequences that this phenomenon can entail, interviewing professionals and academics in the sector who had already dealt with this problem for the Eurozone.

As already discussed in 2017 at ABI Risk and Supervision [14] and, more recently, in the webinar "Valuation risk management in the time of the crisis" (July 14, 2020), many of the problems introduced by negative interest rates in the field of quantitative finance have already been analyzed. The main considerations are summarized below, referring for each of them to the related studies already published:

\section{- Anomalies in the shape of interest rates term structure}

The particularly low level of interest rates has led to anomalies in the shape of the term structure and introduced strong discontinuities mainly due to the high illiquidity recorded in the financial markets. This phenomenon has led to problems in modeling the term structure used for simulations and what-if scenarios in the field of risk management and in the actuarial context [7]. The most popular traditional regressive models used for this purpose are: Nelson-Siegel (1987) [27], Svensson (1994) [22] and de Rezende-Ferreira (2011) [12]. The anomalies in the shape of the interest rates term structures worsened the statistical fit and introduced numerical instability in the estimation of the parameters of the regressive models. In these critical cases, a solution that enables to reach high performance in terms of statistical measures can be found in the implementation of a Machine Learning methodology such as a supervised neural network: a Radial Basis Function [5] or a static feed-forward shallow network [8].

\section{- Interest rates options}

Before the advent of negative interest rates, the most widespread pricing framework in finance, especially among practitioners and professionals, for the pricing of vanilla options on interest rates (Caps and Floors) was undoubtedly the log-normal one. All the main info-providers provided modules to traders for calculating the price and estimating the Greeks in accordance with Black's reference model. With the particular critical conditions of today's markets, this pricing framework cannot work due to the impossibility of calculating the terms of the formula for caplets and floorlets that involve the logarithmic function (referring to traditional notations: $d_{1}$ e $d_{2}$ ) [4].

Therefore, for more than four years in the Eurozone and for only a few months for derivatives denominated in USD, the default pricing model for standard options written on interest rates (i.e. Black log-normal model) has been replaced with the normal model through the Bachelier closed formula. Due to the change of model made by all the main info-providers, including Bloomberg ${ }^{\circledR}$, a valuation and pricing risk was indoubtly introduced. 
A logical consequence of replacing the valuation model is the discrepancy in the Greeks estimation in accordance with the new reference pricing framework. Recalling that the Greeks are computed by estimating the derivatives of the price with respect to a key parameter of the theoretical valuation model, it is clear that if the fair value estimation framework is replaced, the same change will be reflected on the sensitivity measures, regardless of whether these derivatives can be analytically calculated using a closed formula or numerically adopting an integration scheme, such as a finite difference method or a lattice approach. The study [16] shows both the analytical derivation of the Greek for the more important interest rate derivatives and how their replacement impacts from a hedging perspective.

\section{- Changing the implied volatility surfaces}

Implied volatility surfaces are an essential tool for the OTC interest rate options valuation in line with the future expectations of financial markets, as well as being a tool used by traders for their investment choices [15]. From a quantitative point of view, they are derived from the numerical inversion of the theoretical pricing formula using a goal seeking algorithm: starting from the premiums actively quoted on the market, the implicit volatility is obtained. It is clear that this calculation depends on the assumptions made on the underlying valuation model, therefore the reference volatility surfaces have changed, leading to new market quotes conventions (from percentage to basis points), the need for a new sensitivity to the data by the market agents, a different data pipe-line feeding the automatic pricing systems, ...

\section{- Surface Reconstruction}

Under the hypothesis of continuing to use the same log-normal pricing model (for example for contractual constraints related to deals signed decades ago), the problem of managing missing data within the implied volatility surfaces becomes a priority.

Since it is not possible to perform the numerical inversion of the pricing formula deriving from the log-normal framework for options with underlying negative rates, the data missing problem is not a local problem, for which there are various statistical methods that are easy and quick to implement (such as bidimensional interpolation), but a global one: it involves the reconstruction of entire sections of the surface [9]. The surface reconstruction problem can be solved, for example, by implementing machine learning techniques. Paper [10] shows an example of a surface reconstruction for the ATM implied volatilities in application of swaptions pricing through a Non linear Principal Component Analysis (NLPCA) carried out using an Artificial Neural Network (AANN) Autoencoder.

\section{- Violation of fundamental properties for American call options written on an equity underlying with no pay-out}

The advent of negative interest rates leads to the invalidation of many theoretical and mathematical properties related to options, including a property heavily used in the early exercise valuation. Under the condition of negative interest rates, the value of an American call, written on an underlying that does not pay dividends, is no longer equal to its European counterpart [6]. The majority of commercial calculation routines used for the evaluation of standard American options are based on a set of pricing formulas, known in the literature as quasi-closed formulas. The most accurate approximated formula is the Bjerksund-Stensland 2002 formula. These approaches carry out an a-priori check on the value used for the continuous dividend yield: if this is not positive, they return the corresponding value of the European option. This control does not take into account that the optionality due to its possible early exercise has, in interest rate negative environments, a potentially non-zero value.

Therefore, as discussed in the latest FINMAB (Finance MathWorks Advisory Board) in Frankfurt, in order to adjust the valuation taking into account this extreme condition, it is necessary to take into consideration, regardless the pay-out of the underlying, a numerical techniques such as stochastic trees or a free-boundary PDE able to correctly price the early exercise feature.

The purpose of this article is to add to the scientific contributions reported in this brief introduction by examining the problems emerged in the prospective simulations of short term rates after the advent of negative interest rates.

We will therefore analyze the most widespread stochastic differential equations in the literature that allow to perform this task and we will discuss the potential problems that make them unusable in the current anomalous financial context and, once a subgroup has been identified for which it is possible to apply a numerical integration scheme, we will proceed to analyze the process of estimating the parameters of these dynamics.

After having re-examinated the calibration techniques derived by the application of the maximum likelihood method on the observed market data, we will discover that not all the SDEs of the subgroup for which the application of a numerical integration scheme was feasible lead to consistent or admissible results.

The Hull-White model reveals to have robust dynamics both in the numerical integration part and in the tuning of its characteristic parameters during the market calibration phase.

\section{Short rate dynamics}

The most widespread stochastic differential equations for the simulation of the short rate are:

[V] Vasicek [23]

$d r_{t}=k\left[\Theta-r_{t}\right] d t+\sigma d W_{t}$ 
[D] Dothan [13]

$d r_{t}=a r_{t} d t+\sigma r_{t} d W_{t}$

[CIR] Cox - Ingersoll - Ross [11]

$d r_{t}=k\left[\Theta-r_{t}\right] d t+\sigma \sqrt{r_{t}} d W_{t}$

[EV] Exponential Vasicek [3]

$d r_{t}=r_{t}\left[\eta-a \ln r_{t}\right] d t+\sigma r_{t} d W_{t}$

[HW] Hull - White [17]

$d r_{t}=k\left[\theta_{t}-r_{t}\right] d t+\sigma d W_{t}$

[BDT] Black - Derman - Toy [1]

$d \ln r_{t}=\left[\theta_{t}+\frac{\sigma_{t}^{\prime}}{\sigma_{t}} \ln r_{t}\right] d t+\sigma_{t} d W_{t}$

[BK] Black - Karasinski [2]

$d r_{t}=r_{t}\left[\eta_{t}-a \ln r_{t}\right] d t+\sigma r_{t} d W_{t}$

[MM] Mercurio - Moraleda [20]

$d r_{t}=r_{t}\left[\eta_{t}-\left(\lambda-\frac{\gamma}{1+\gamma_{t}}\right) \ln r_{t}\right] d t+\sigma r_{t} d W_{t}$

The mathematical notations used in the equations are the same adopted by Brigo-Mercurio [3]. With the reference of the EURIBOR time-series, the level of the short rate, $r_{t}$ is deeply negative.

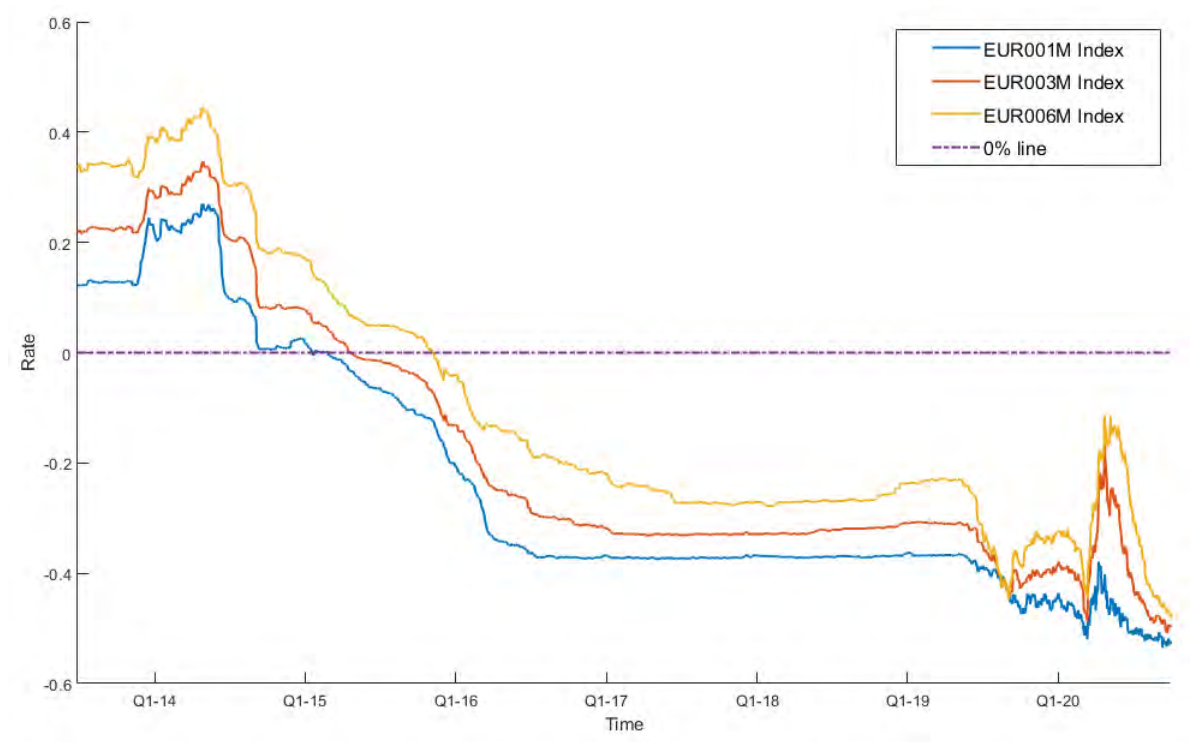

Figure 1. EURIBOR time series. Tenor: 1 month, 3 months and 6 months - Source: Bloomberg ${ }^{\circledR}$

The starting value of the rate used for the SDE numerical integration is equal to the level recorded by the market for the initial starting time: $r_{t=0}=r_{M K T}$.

Given that the initial value is negative, the majority of the listed models cannot be used for the simulation of the short-rate. The reason is simple and clear: many dynamics involve the presence of mathematical operators which cannot work with negative real numbers (logarithm and square roots). These correspond to the red terms in the above list.

Among the traditional models, Vasicek [V], Dothan [D] and Hull-White [HW] SDEs can be integrated using a traditional numerical integration scheme (for instance Euler-Maruyama or Milstein method).

\section{SDE parameters estimation}

The aim of this paragraph is to analyze the most widespread calibration methodologies necessary for the determination of the characteristic parameters for the SDEs which can be numerically solved: Vasicek [V], Dothan [D] and Hull-White [HW] models. The paragraph provides the theoretical and practical evidences for which the HW model has proved to be more suitable to be implemented for conducting prospective short-rate simulations. 
In particular, Vasicek calibration fails under low-level rates because the canonical tuning method leads to the estimation of two negative SDE characteristic parameters. As a result the long-run rate will not converge. In the case of the Dothan model, the two parameters which rule the dynamics cannot be found because in the zero-coupon bond closed formula used for pricing, there is a mathematical operator which does not work with negative numbers. The Hull-White model has a bigger flexibility to match the current interest rate structure and this feature allows to calibrate the parameter using a larger set of financial instruments, such as caps, floors, swaptions and zero-coupon bonds as well. As a result, after the preliminary feasibility to the SDE integration, even the parameter estimation phase proves to be robust also in an extreme market context.

\subsection{Vasicek calibration problem}

In order to estimate the parameters of the stochastic models, we use a deterministic expression for the expected theoretical value for a financial instrument in accordance with the selected dynamics and, therefore, we find the parameters that better fit the quoted realized price for that financial instrument (or set of financial instruments).

Given that the market provides a set of quotes for these instruments, the characteristic parameters of the stochastic model can be calibrated starting from the values observed on the market using the Maximum Likelihood Principle.

In the case of the Vasicek model, there is a set of closed formulas that allow direct tuning of the parameters of the dynamics $(\theta$, $k$ e $\sigma$ ) starting from the rates observed on the market [3], [23].

$$
\begin{aligned}
& \hat{\theta}=\frac{S_{y} S_{x x}-S_{x} S_{x y}}{n\left(S_{x x}-S_{x y}\right)-\left(S_{x}^{2}-S_{x} S_{y}\right)} \\
& \hat{k}=-\frac{1}{\delta} \ln \frac{S_{x y}-\Theta S_{x}-\Theta S_{y}+n \Theta^{2}}{S_{x x}-2 \Theta S_{x}+n \Theta^{2}}(10) \\
& \hat{\sigma}^{2}=\frac{2 k\left[S_{y y}-2 \alpha S_{x y}+\alpha^{2} S_{x x}-2 \Theta(1-\alpha)\left(S_{y}-\alpha S_{x}\right)+n \Theta^{2}(1-\alpha)^{2}\right]}{n\left(1-\alpha^{2}\right)}
\end{aligned}
$$

Where:

$$
\alpha=\exp (-k \delta), S_{x}=\sum_{i=1}^{n} r_{i-1}, S_{y}=\sum_{i=1}^{n} r_{i}, S_{x x}=\sum_{i=1}^{n} r_{i-1}^{2}, S_{y y}=\sum_{i=1}^{n} r_{i}^{2}, S_{x y}=\sum_{i=1}^{n} r_{i-1} r_{i} \text { and } \delta \text { is the tenor of the rates. }
$$

The example below demonstrates how this procedure does not prove to be robust in extreme market conditions.

Consider the 3-month EURIBOR interest rates term as of $30^{\text {th }}$ June 2017 shown in Table 2 and obtained from the market par rates (Table 1). By calibrating the parameters that rule the Vasicek dynamics using the set of equations (9), (10) and (11) we obtain the values: $\hat{\theta}=-0.0436, \hat{k}=-0.0257$ e $\hat{\sigma}=3.1676 \cdot 10^{-4}$

The scientific literature reports that the dynamics model can be considered reliable if all three parameters are positive.

Negative interest rates have led to a negative mean-reversion rate $k$, which in turn leads to an asymptotic divergence of the short-term rate (Figure 2)

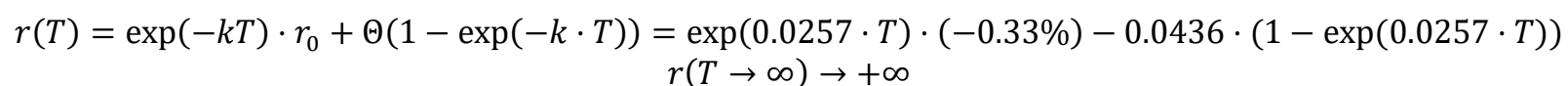

\begin{tabular}{|c|c|c|c|}
\hline Instrument & Bloomberg $^{\circledR}$ Codifier & Par Rates (Mid Value) & Day Basis \\
\hline Deposit & EONIA Index & -0.350 & ACT/360 \\
\hline Deposit & EUDR2T Index & -0.410 & ACT/360 \\
\hline Deposit & EUR001W Index & -0.379 & ACT/360 \\
\hline Deposit & EUR002W Index & -0.373 & ACT/360 \\
\hline Deposit & EUR001M Index & -0.373 & ACT/360 \\
\hline Deposit & EUR002M Index & -0.342 & ACT/360 \\
\hline Deposit & EUR003M Index & -0.331 & ACT/360 \\
\hline Deposit & EUR006M Index & -0.271 & ACT/360 \\
\hline Deposit & EUR009M Index & -0.197 & ACT/360 \\
\hline Deposit & EUR012M Index & -0.156 & ACT/360 \\
\hline Swap & EUSW2V3 BGN Curncy & -0.202 & $30 / 360$ \\
\hline Swap & EUSW3V3 BGN Curncy & -0.085 & $30 / 360$ \\
\hline Swap & EUSW4V3 BGN Curncy & 0.038 & $30 / 360$ \\
\hline Swap & EUSW5V3 BGN Curncy & 0.166 & $30 / 360$ \\
\hline Swap & EUSW6V3 BGN Curncy & 0.294 & $30 / 360$ \\
\hline Swap & EUSW7V3 BGN Curncy & 0.427 & $30 / 360$ \\
\hline Swap & EUSW8V3 BGN Curncy & 0.557 & $30 / 360$ \\
\hline Swap & EUSW9V3 BGN Curncy & 0.681 & $30 / 360$ \\
\hline Swap & EUSW10V3 BGN Curncy & 0.796 & $30 / 360$ \\
\hline
\end{tabular}

Table 1. Market Yield curve. Tenor: EURIBOR 3 months. Reference Date: 30th June 2017 (Source Bloomberg) 


\begin{tabular}{|c|c|c|c|}
\hline Start Date & End Date & Zero Rates & Discount Factors \\
\hline $06 / 30 / 2017$ & $07 / 03 / 2017$ & -0.35423738 & 1.000029168 \\
\hline $07 / 03 / 2017$ & $07 / 04 / 2017$ & -0.369389988 & 1.000040557 \\
\hline $07 / 04 / 2017$ & $07 / 11 / 2017$ & -0.378395184 & 1.00011426 \\
\hline $07 / 04 / 2017$ & $07 / 18 / 2017$ & -0.375692917 & 1.000185639 \\
\hline $07 / 04 / 2017$ & $08 / 04 / 2017$ & -0.376596976 & 1.000361868 \\
\hline $07 / 04 / 2017$ & $09 / 04 / 2017$ & -0.347653817 & 1.000629928 \\
\hline $07 / 04 / 2017$ & $10 / 04 / 2017$ & -0.336602074 & 1.000887196 \\
\hline $07 / 04 / 2017$ & $01 / 04 / 2018$ & -0.276594944 & 1.001427645 \\
\hline $07 / 04 / 2017$ & $04 / 04 / 2018$ & -0.20213019 & 1.001542258 \\
\hline $07 / 04 / 2017$ & $07 / 04 / 2018$ & -0.160458749 & 1.001624793 \\
\hline $07 / 04 / 2017$ & $07 / 04 / 2019$ & -0.202868958 & 1.004092105 \\
\hline $07 / 04 / 2017$ & $07 / 06 / 2020$ & -0.08604541 & 1.002602363 \\
\hline $07 / 04 / 2017$ & $07 / 05 / 2021$ & 0.036960075 & 0.998516897 \\
\hline $07 / 04 / 2017$ & $07 / 04 / 2022$ & 0.165512383 & 0.991742847 \\
\hline $07 / 04 / 2017$ & $07 / 04 / 2023$ & 0.29475367 & 0.982456191 \\
\hline $07 / 04 / 2017$ & $07 / 04 / 2024$ & 0.429774866 & 0.970357931 \\
\hline $07 / 04 / 2017$ & $07 / 04 / 2025$ & 0.5629423 & 0.955996167 \\
\hline $07 / 04 / 2017$ & $07 / 06 / 2026$ & 0.69109287 & 0.939755663 \\
\hline $07 / 04 / 2017$ & $07 / 05 / 2027$ & 0.811061357 & 0.92225464 \\
\hline
\end{tabular}

Table 2. Interest rates term structure. Zero Rates are stripped using market par-rates reported in Table 1

The parameter that regulates the mean-reversion, $k$, is negative and constant: in the presented case it produces an asymptotic divergence of the short-term rate which cannot be reached at the long-term value $\theta[14]$.

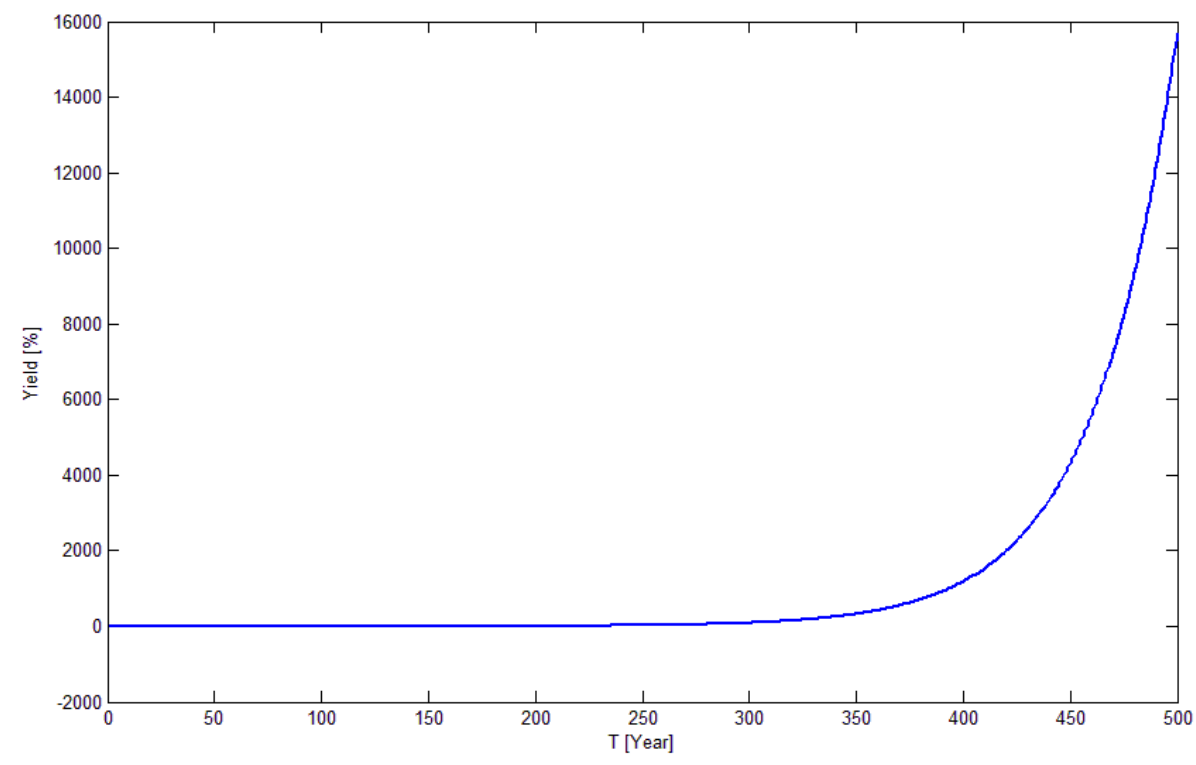

Figure 2. Divergence of the short rate in the long run caused by negative interest rates

\subsection{Dothan calibration problem}

Dothan's model is the only log-normal short rate model to have an analytical formula for pure zero-coupon bonds, $P(t, T)$ [3], [13].

$P(t, T)=\frac{\overline{r^{P}}}{\pi^{2}} \int_{0}^{\infty} \sin (2 \sqrt{\bar{r}} \sinh (y)) \int_{0}^{\infty}(f(z) \sin (y z) d z d y)+\frac{2}{\Gamma(2 \mathrm{p})} \overline{r^{P}} K_{2 p}(2 \sqrt{\bar{r}})$

Where:

$f(z)=\exp \left[\frac{-\sigma^{2}\left(4 p^{2}+z^{2}\right)(T-t)}{8}\right] z\left|\Gamma\left(-p+i \frac{z}{2}\right)\right|^{2} \cosh \left(\frac{\pi z}{2}\right), \bar{r}=\frac{2 r(t)}{\sigma^{2}}, p=\frac{1}{2}-a$ and $K_{q}$ denotes the modified Bessel function of the second kind of order $q$. 
Its calibration therefore takes place using the maximum likelihood method starting from the set of zero-rates observed by the term structure of the reference rate.

As already observed in the term structures shown in Table 2, a significant sample of rates that can be used for calibration is negative, therefore the closed formula from which it is possible to calculate the value of a zero-coupon in accordance with the Dothan model is not applicable [14].

In fact, if $r(t)<0$ then $\bar{r}=\frac{2 r(t)}{\sigma^{2}}<0$ and, consequently, the amount in the squared root cannot be computed.

\subsection{Hull - White calibration}

Compared to Vasicek's model, the dynamics proposed by Hull-White sets $\Theta$ as a function of time.

This assumption allows on the one hand to significantly improve the fit of the interest rates term structure and on the other hand to have a greater range of financial instruments that can be used for the tuning of the stochastic differential equation parameters [18], [19].

In fact, the parameters of the model can be calibrated starting from the premiums of the most popular and listed options linked to the interest rate such as caps and floors.

According to this dynamics, the short-rate can be represented as follows [3]:

$\left\{\begin{array}{c}d r=[\Theta(t)+\alpha r] d t+\sigma d W_{t} \\ \Theta(t)=\frac{\partial f^{M}(0, t)}{\partial T}+\alpha f^{M}(0, t)+\frac{\sigma^{2}}{2 \alpha}(1-\exp (-2 \alpha t))\end{array}\right.$

Where $\alpha$ and $\sigma$ are the mean reversion and the volatility of the model, respectively, $\Theta(t)$ is chosen in order to exactly adapt the interest rate term structure on the evaluation date and $f^{M}(0, t)$ are the instantaneous market forward rates at time 0 for the maturity $t$.

Eq. (13) can be discretized using a trinomial stochastic tree, which is commonly used for pricing exotic instruments: this integration scheme has the parameters $\alpha$ e $\sigma$ and have to be tuned in accordance with the market prices for cap and floor [3].

The analytical tractability of the model allows to derive a closed formula that expresses the theoretical value of the caps and floors: this is an interesting property as it allows greater speed in parameter tuning without necessarily having to code a recursive routine call of the stochastic tree itself.

$$
\begin{aligned}
& \operatorname{Cap}(t, \tau, N, X)=N \sum_{i=1}^{n} P\left(t, t_{i-1}\right) \Phi\left(-h_{i}+\sigma_{P}^{i}\right)-\left(1+X \tau_{i}\right) P\left(t, t_{i}\right) \Phi\left(-h_{i}\right) \\
& F \operatorname{loor}(t, \tau, N, X)=N \sum_{i=1}^{n}\left(1+X \tau_{i}\right) P\left(t, t_{i}\right) \Phi\left(h_{i}\right)-P\left(t, t_{i-1}\right) \Phi\left(h_{i}-\sigma_{P}^{i}\right)
\end{aligned}
$$

With:

$$
\left\{\begin{array}{c}
\sigma_{P}^{i}=\sigma \sqrt{\frac{1-\exp \left[-2 \alpha\left(t_{i-1}-t\right)\right]}{2 \alpha}} B\left(t_{i-1}, t_{i}\right)=\sigma \sqrt{\frac{1-\exp \left[-2 \alpha\left(t_{i-1}-t_{i}\right)\right]}{2 \alpha}} \frac{1}{\alpha}\left\{1-\exp \left[-\alpha\left(t_{i}-t_{i-1}\right)\right]\right\} \\
h_{i}=\frac{1}{\sigma_{P}^{i}} \ln \left[\frac{P\left(t, t_{i}\right)\left(1+X \tau_{i}\right)}{P\left(t, t_{i-1}\right)}\right]+\frac{\sigma_{P}^{i}}{2}
\end{array}\right.
$$

Where:

$X$ is the strike of the option

$N$ is the nominal value

$\tau$ is the year fraction between each caplet (/floorlet)

$P\left(t, t_{i}\right)$ is the discount factor

$\Phi$ is the standardized normal cumulative distribution function

$\alpha$ and $\sigma$ are the mean reversion and the volatility of the Hull-White tree to find

This model allows to deal with negative interest rates both in the integration phase of the stochastic differential equation and in the parameter estimation phase.

A calibration is carried out starting from the normal implied volatility of caps quoted by the market on $30^{\text {th }}$ June 2017 (Figure 3). 


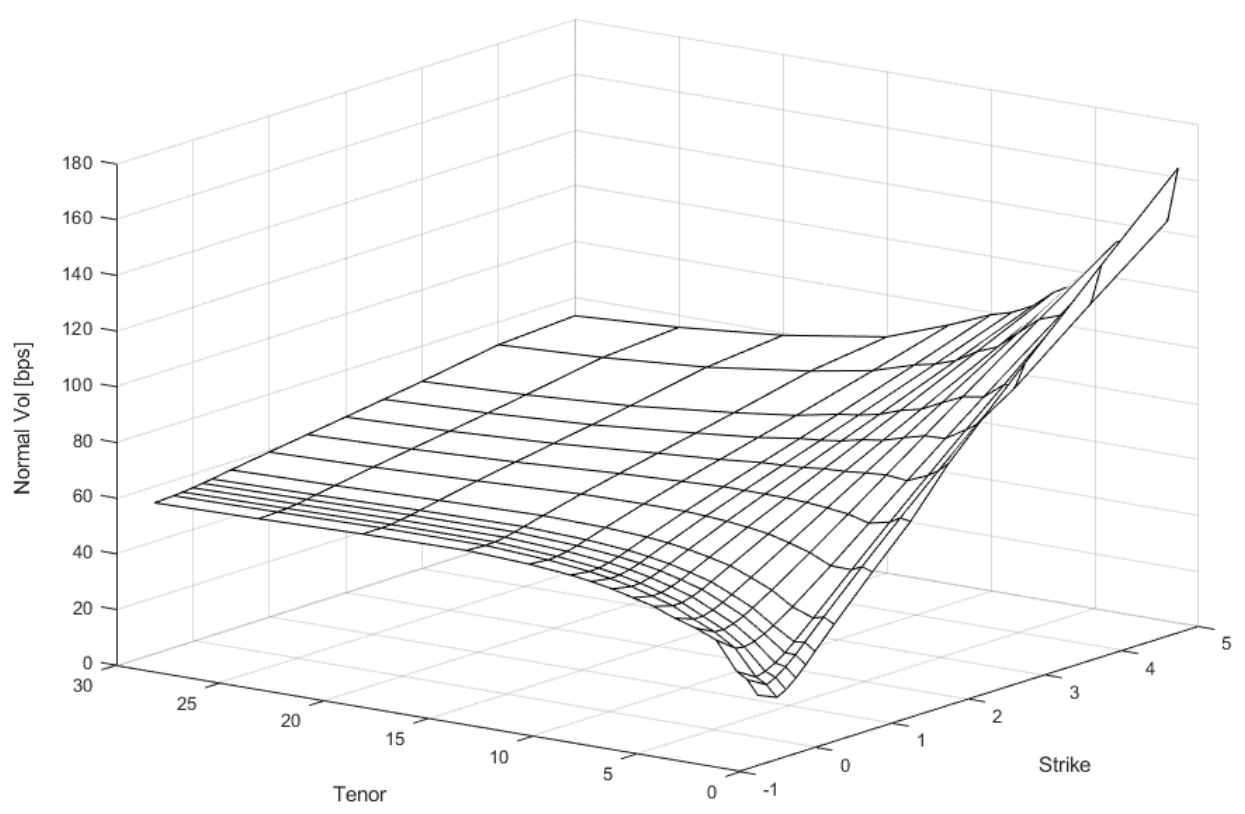

Figure 3. Cap Normal Implied Volatility. Reference Date: 30th June 2017. Source: Bloomberg®

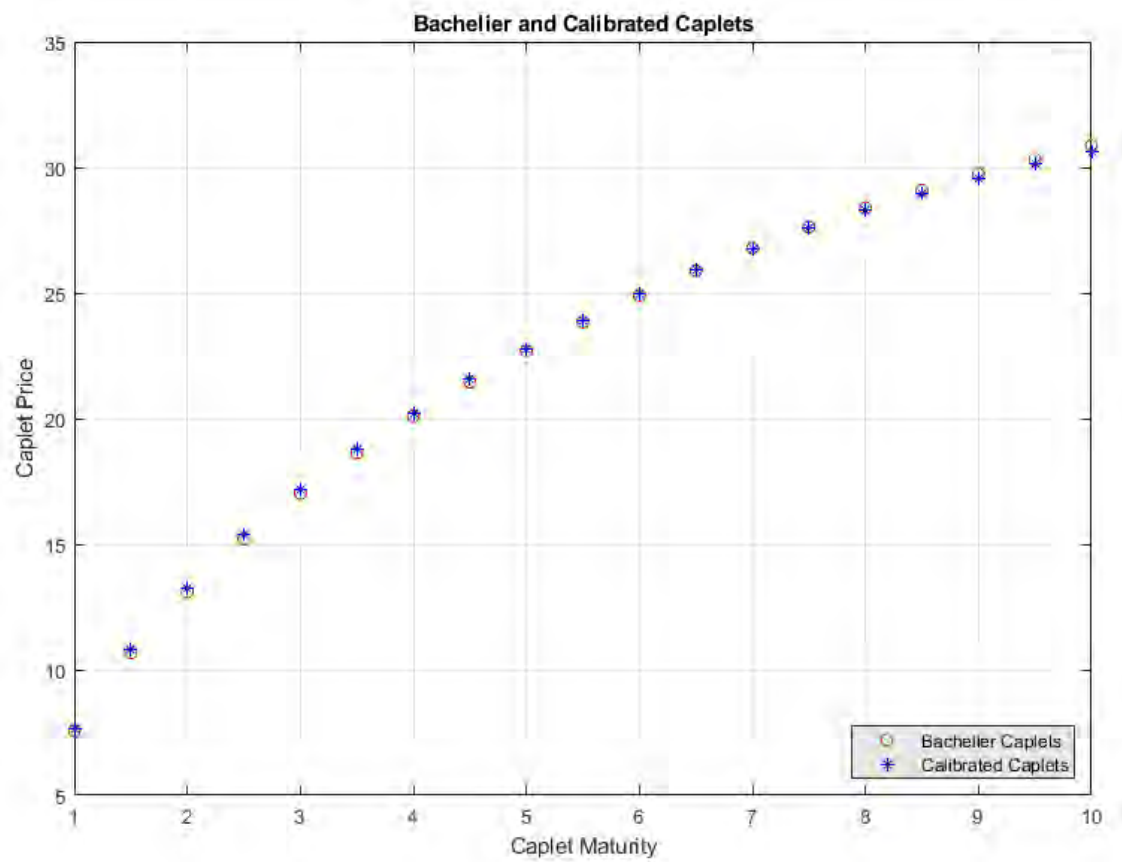

Figure 4. Hull White parameter estimation

In the case of the Hull-White model, no anomalies were found in the set of pricing formulas used to calibrate the dynamics. These considerations make the HW a robust and reliable model even in a context of negative rates [14].

\section{Conclusions}

The article highlighted some critical issues introduced by negative interest rates in the most popular models for representing the short rates.

The problems caused by these were found not only in the definition of the descriptive dynamics of motion, but also in the calibration procedure of the SDE characteristic parameters.

The Hull-White dynamics proved to be robust and reliable: although the analysts in the 90's made several criticisms about this model, since it did not guarantee the positivity of rates in future simulations, in the anomalous current market context it must be strongly taken into consideration precisely for this aspect. 


\section{References}

[1] F. Black, E. Derman, W. Toy - "A one-factor model of interest rates and its application to treasury bond options" - Financial Analyst Journal, 33-39 (1990)

[2] F. Black, P. Karasinski - "Bond and Option pricing when short rates are lognormal” - Financial Analyst Journal, 52-59 (1991)

[3] D. Brigo, F. Mercurio - "Interest Rate Models: Theory and Practice with Smile, Inflation and Credit" - Springer Finance (2006)

[4] G. Burro, P. G. Giribone, S. Ligato, M. Mulas, F. Querci - "Negative interest rates effects on option pricing: Back to basics?" - International Journal of Financial Engineering Vol. 4, N. 2 (2017)

[5] A. Cafferata, P. G. Giribone, M. Neffelli, M. Resta - "Yield curve estimation under extreme conditions: do RBF networks perform better?

"- Chapter 22 in book: "Neural Advances in Processing Nonlinear Dynamic Signals" - Springer (2019)

[6] A. Cafferata, P. G. Giribone, M. Resta - "The effects of negative nominal rates on the pricing of American calls: some theoretical and numerical insights " - Modern Economy Vol. 9, N. 6 (2017).

[7] A. Cafferata, P. G. Giribone, M. Resta - "Interest rates term structure models and their impact on actuarial forecasting" - QFW18: Quantitative Finance Workshop 2018 (UniRoma3 - Rome)

[8] O. Caligaris, P. G. Giribone - "Modellizzare la curva dei rendimenti mediante metodologie di apprendimento artificiale: analisi e confronto prestazionale tra le tecniche regressive tradizionali e le reti neurali" - AIFIRM Magazine Vol. 10, N. 3 (2015)

[9] O. Caligaris, P. G. Giribone, S. Ligato - "Applicazione delle reti neurali feed-forward per la ricostruzione di superfici di volatilità" AIFIRM Magazine Vol. 10, N. 2 (2015)

[10] O. Caligaris, P. G. Giribone, M. Neffelli - "Ricostruzione di superfici di volatilità mediante l'utilizzo di reti neurali auto-associative: un caso studio basato sull'analisi non lineare delle componenti principali " - Risk Management Magazine Vol. 12, N. 3 (2017)

[11] J. C. Cox, J. E. Ingersoll, S. A. Ross - "A theory of the term structure of interest rates" - Econometrica, 53, 385-407 (1985)

[12] R. B. de Rezende, M. S. Ferreira - "Modeling and forecasting the yield curve by an extended Nelson-Siegel class of model: a quantile autoregressive approach" - Journal of Forecasting, 32, 111-123 (2011).

[13] L. U. Dothan - "On the term structure of interest rates" - Journal of Financial Economics, 6, 59-69 (1978)

[14] P. G. Giribone - "Il problema dei tassi d'interesse negativi nel pricing e nell'hedging" - Atti della Conferenza ABI (Associazione Bancaria Italiana) "Risk and supervision" (2017)

[15] P. G. Giribone, S. Ligato - "Considerazioni sullo stato attuale della valorizzazione delle opzioni cap e floor aventi come parametro di riferimento il tasso EURIBOR" - AIAF (Associazione Italiana Analisti Finanziari) Magazine, Vol. 99 (2016)

[16] P. G. Giribone, S. Ligato, M. Mulas - "The effects of negative interest rates on the estimation of option sensitivities: the impact of switching from a log-normal to a normal model" - International Journal of Financial Engineering Vol. 4, N. 1 (2017)

[17] J. Hull, A. White - "Pricing interest rate derivative securities" - Review of financial studies, 3(4), 573-592 (1990)

[18] J. Hull, A. White - "One-factor interest rate models and the valuation of Interest rate derivative securities" - Journal of Financial and Quantitative Analysis, 28, 235-254 (1993)

[19] J. Hull, A. White - "Numerical Procedures for implementing Term Structure Models: Single-Factor Models" - The Journal of Derivatives, 2, 7-16 (1994)

[20] F. Mercurio, J. M. Moraleda - “An analytically tractable interest rate model with humped volatility” - European Journal of Operational Research, 120, 205-214 (2000)

[21] C. R. Nelson, A. F. Siegel - "Parsimonious modeling of the yield curve" - The Journal of business, 4, 473-489 (1987)

[22] L. E. O. Svensson - "Estimating and interpreting forward interest rates: Sweden 1992-94" - IMF Working paper (1994)

[23] O. Vasicek - "An equilibrium characterization of the term structure" - Journal of Financial Economics, 5, 177-188 (1977) 


\section{Blockchain securitization: an innovative technology to boost asset liquidity}

Paolo Fabris (Partner Avantage Reply); Valerio Begozzi (Manager Avantage Reply); Angelo Santarossa (Manager Avantage Reply); Francesco Dammacco (Collaborator Avantage Reply); Gianmarco Fagiani (Consultant Avantage Reply); Riccardo Rostagno (Consultant Blockchain Reply); Chiara Frigerio (Università Cattolica del Sacro Cuore di Milano)

Article submitted to double-blind peer review, received on 10th November 2020 and accepted on 18 th December 2020

\section{Abstract}

The main aim of this paper is to show the potential benefits for the Securitisation process, both in terms of the setup of operations and in the entire product life cycle, derived from the adoption of the Blockchain Technology. For this purpose, we focused on the different aspects, starting from the securitisation market in which we analysed the causes of the decrease in the number of operations occurred in the last decade, although there still is a strong need to securitise some types of assets such as NPL's and Trade Receivables. Specifically, for these two types of assets we represent the securitisation process with the As-Is limits and improvements made possible by the blockchain technology application. Along this "path" we have deepened some key aspects of the blockchain and how the application of this technology may help Financial Institutions to experience a strong reduction in operational risks and costs, liquidity risk and credit risk on managing the underlying assets. At the same time, the new framework might give to a wider category of individuals access to a product that otherwise would have been available only to a limited number of more sophisticated investors, tapping from more structured, controlled and certified information. All this being achieved at no additional costs but rather with stronger and more organized and transparent product structures.

\section{Keywords}

Blockchain Benefits, Securitisation Process, STS Framework, NPL, Trade Receivable, Illiquid Asset, Data Quality, Data Security, Data Certification (Oracles), DLT, Smart Contract

\section{Introduction}

\subsection{Illiquid Assets}

Over the past few years, the interest towards the management and monitoring of illiquid investments has increased between both financial and private market participants, as they seek for higher risk adjusted returns after the urgent monetary stimulus, introduced during the Financial Crisis, which pushed high quality interest rates to record lows. Therefore, it is significant to highlight the main aspects which discriminate them from traditional financial assets.

The main sources of illiquidity are (TAFOLONG \& TEDONGAP, 2017):

- Transaction costs: which usually affect more short-term investments than long-term investments and have greater influence on trading frequency

- Market organization: the absence of a secondary market could cause the lack of trading partners at any given point of time which give rise to liquidation costs whereas complexity and weak comparability related to transactions may lead to a significant distortion of asset characteristics

- Discrepant evaluations for the same asset: difference in opinions about the real/fair value of an asset as a result of:

o information asymmetry - heterogeneous information between traders and their respective counterparties (AKERLOF, 1970)

o divergence in expectations - different forecasts of the future cash flow and riskiness

o divergence in used possibilities - the interest in assets is influenced by individual needs and valuations (i.e., arts/collectible)

The market value of the main categories of illiquid assets was about $\$ 400$ trillion in 2018 , with the dominance of allocations in real estate class taking about half of the market share, followed by credit, derivatives, equity shares and residual categories of arts/collectibles and cryptocurrencies, as reported in the figure below (CeTIF, 2020).

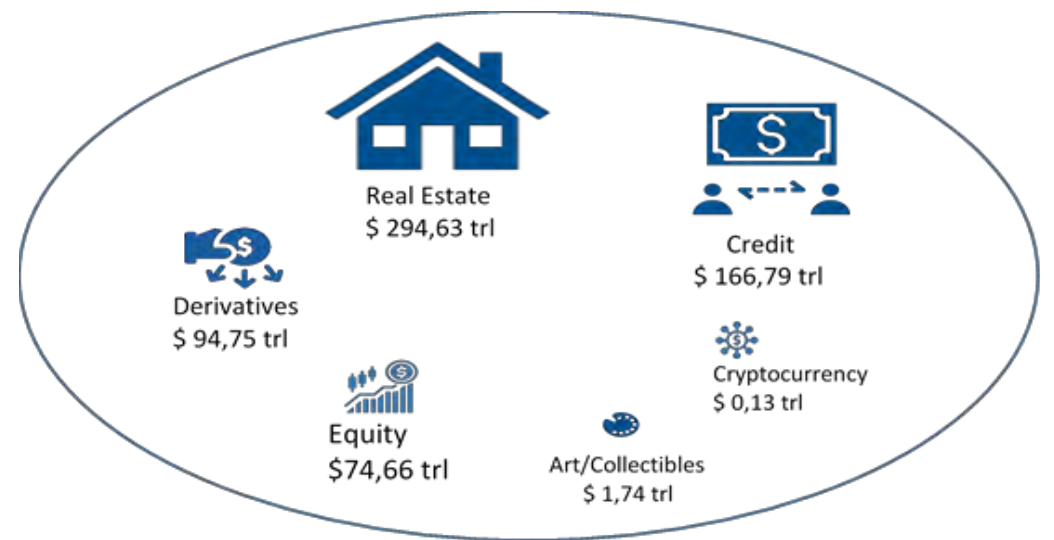

Fig. 1 - Global Asset Class Capitalization in 2018. Source: CeTIF 


\subsection{Securitisation Market and process constraints}

Converting these illiquid assets into tradeable securities, which enables a financial institution to efficiently allocate capital and risks and increase liquidity, is an essential task especially during uncertain economic scenarios. To achieve this goal, the prevalent mechanism applied throughout the recovery period after the financial crisis was Securitisation.

Securitisation operated as an effective deleveraging instrument in the European banking sector, which held a significant amount of non-performing loans in the balance sheets, freeing capital for further lending. However, as shown in the chart below (AFME, 2020), after the peak of European issuances recorded in 2008 with Eur 818,7 billion, the following years brought a significant drop for Securitisation emissions.

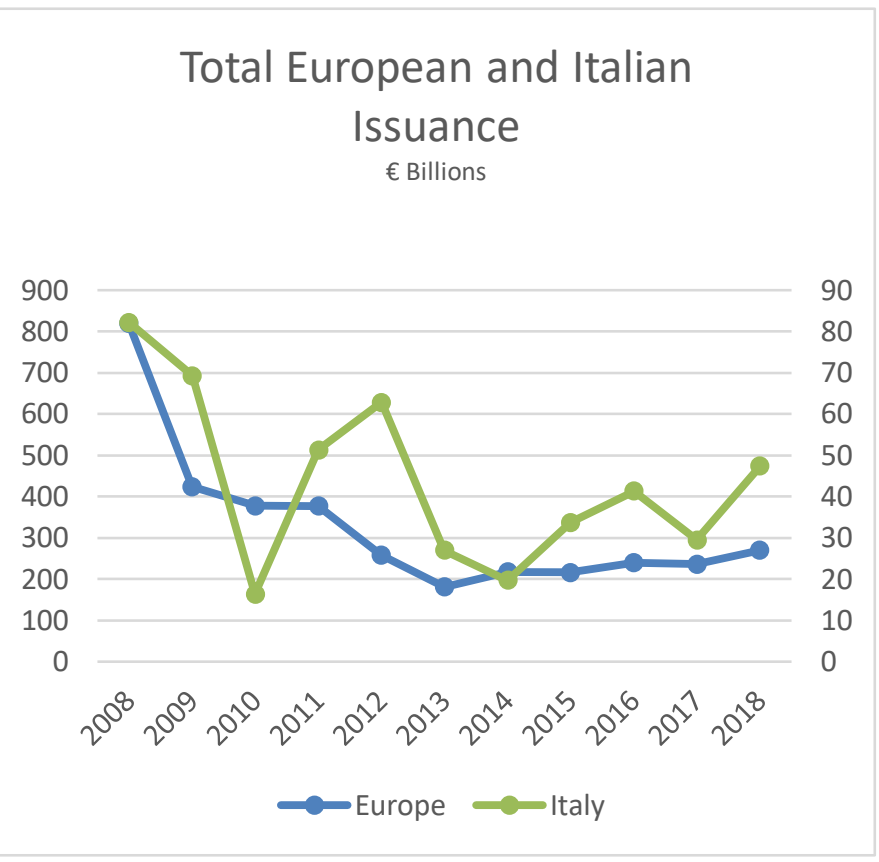

Fig. 3 - Total European Issuance. Source: AFME Securitisation Data Report

\section{European vs Italian Issuance Trend} $\%$

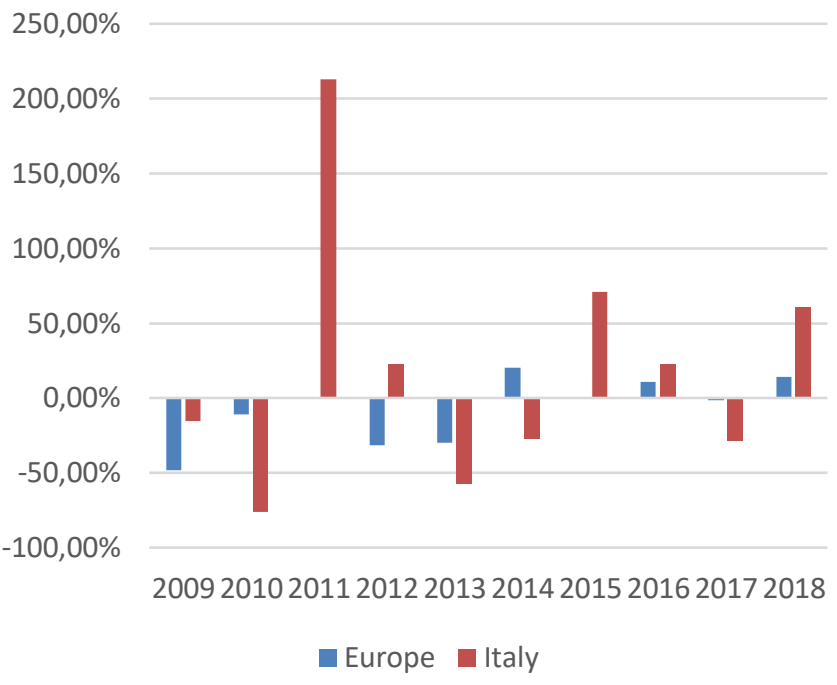

Fig. 2 - European vs Italian Issuance Trend. Source: AFME Securitisation Data Report

The main causes of decrease in issuances lie in the presence of information asymmetries, high entry costs, lack of transparency and complexity.

Information asymmetries is meaning that buyers are uncertain about credit quality as described by sellers which creates a gap between judgments on the potential value of a portfolio.

High due diligence costs are necessary to acquire sufficient information and fill the aforementioned information asymmetry, with a consequent barrier to entry for smaller operators. The securitisation market, specifically of European NPLs, historically has been a limited playing field with few buyers able to obtain significant bargaining power with sellers and are often induced to sell NPL portfolios to comply with limits imposed by the supervisory authorities or to guarantee the expected level of performance consequently impacting sale prices.

Last but not least, in general transparency and complexity are related to the fact that securitisations are often too complex especially for retail investors, whose ability to assess the underlying risk could be weak. The shortage of transparency perceived by the market intensifies the lack of trust which is one of the reasons why there are difficulties to revive investors' appetite for securitisation products, even though the European default rates were low during the Financial Crisis.

The decline can also be attributed to the process itself as these transactions involve many participants with differing roles, objectives and requirements, who face contrasting issues around the storage and the transfer of the information flow. Therefore, securitisation operations currently result as a lengthy and costly process.

In order to overcome these remarkable limits, it is essential to introduce both regulatory amendment and technology enhancement:

\subsection{Regulatory amendment - STS Framework}

European post crisis regulation 2017/2401 - 2017/2042 (EU, European parliament and council, EU Regulation 2017/2401 - 2017/2402, 2017) attempted to revert the trend and sort out some of the constraints described above with a new securitisation framework aimed at strengthening investor's confidence by supporting Simple, Transparent and Standardised securitisations (STS), in contrast to more opaque and complex securitisation transactions, confirming the importance of these financial instruments as an effective funding channel to the economy. The STS framework, came into effect on January 1, 2019, established a preferential capital treatment for those securitisations that comply with a new set of regulatory criteria that should guarantee an easier credit analysis and investor's comprehension with the increase of information disclosure between the parties.

We must point out that, despite regulator efforts, STS framework has not yet produced a huge effect on the market with significant in progress still needed regarding the treatment of synthetic securitisations. In 2019, only Eur 67.6 billion in STS securitisations were issued, which represented $31 \%$ of total issuance in the same year, and the cumulative issuances in the first half of 2020 amounted to Eur 79,3 billion of which Eur 18,4 billion were notified as STS, corresponding to 23,2\% of the total volume. As of Q2 2020, a total of 330 STS securitisations were notified to ESMA, which are mainly focused on Trade Receivables, Auto Loans/Leases and Residential mortgages transactions, as described by the graph below (ESMA, 2020). 


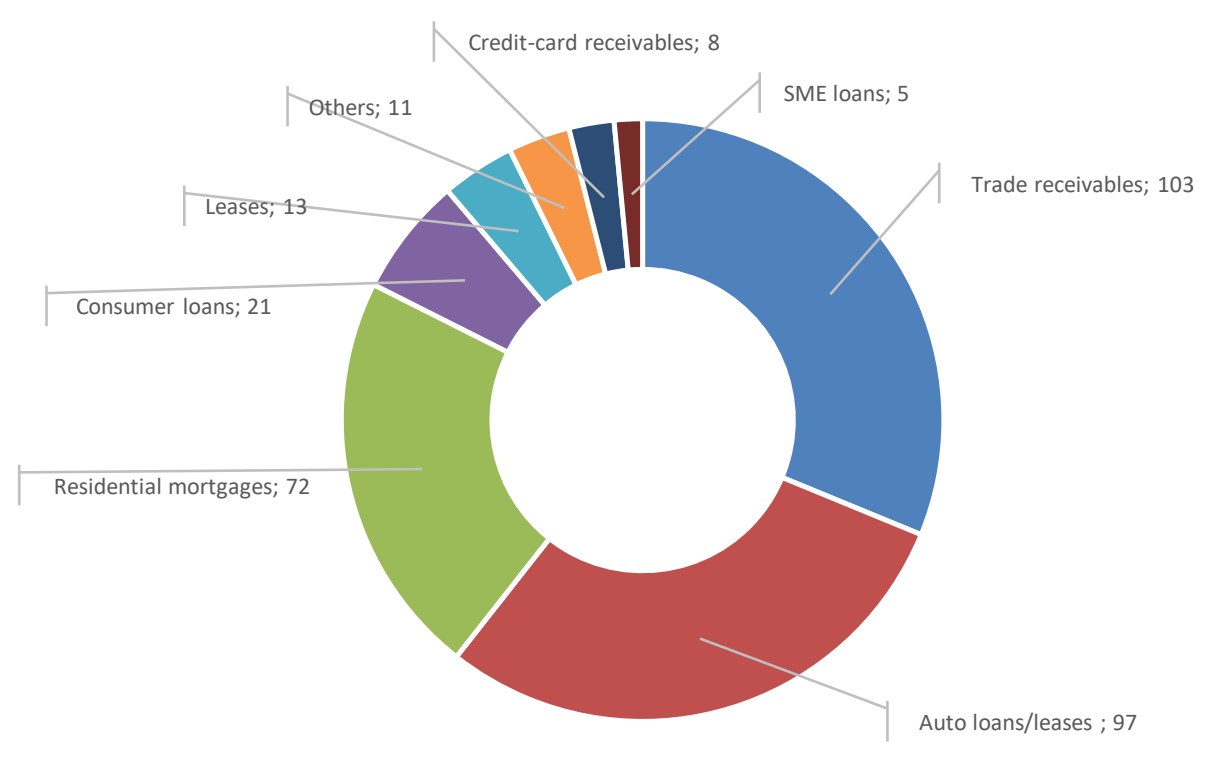

Fig. 4 - STS Notifications by Collateral Type. Source: ESMA

\subsection{Technology Enhancement}

Beside regulatory action, in this context, financial technology represents the keystone to boosting the securitisation market and streamlining asset marketability. The evolution of blockchain, especially, could positively impact the market (TAPSCOTT A., TAPSCOTT D, 2017). bringing numerous advantages including lower costs and faster transactions. As we will see in the next paragraphs, blockchain incorporates a single source of data for all the participants, which can compensate for the existing lack of information connection and standardization within the network from loan origination to primary issuance and through the entire life cycle of the securitisation. This technology, through sharing and synchronization of digital databases, could ultimately materialize time and cost savings, such as increased data availability, greater transparency, elimination of transactional parts and automation. Direct access to information could also facilitate and potentially reduce reporting requirements for both investors and regulators.

\section{Applying blockchain to securitisation}

\subsection{Securitisation Process}

In order to understand how the blockchain technology can improve the securitisation market, it's necessary to focus on the entire process and identifying the activities that can benefit from blockchain (DELOITTE, 2017); (HOFMANN, STREWE, BOSIA, 2018).

Here a brief diagram which synthesizes the main phase of a securitisation process.

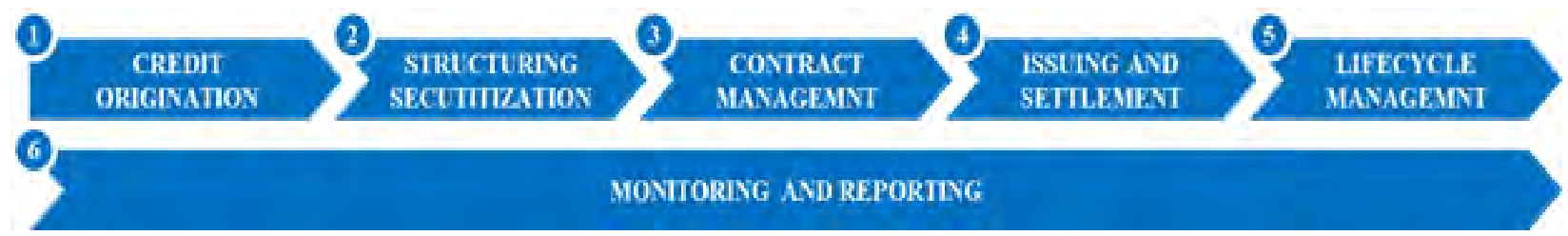

Fig. 5 - Securitization process

Let's have a look in detail.

\subsubsection{Credit origination}

Despite the heavy investments that banks have made in recent years to digitize the underwriting process, many steps are still managed manually and offline.

There are many inefficiencies affecting the credit processes:

- information, where digital, resides on different legacies, they do not have a common storage standard.

- paper information, beyond higher storage costs than digital information, is often useless as it is only physically accessible and its use together with digital data is often complicated and expensive. 
The first step, even if not essential, to reap the greatest benefits from the blockchain is to originate credits digitally.

Today, technology already supports this process through the use of smart contracts and tokens. These digital entities describe all the information generated during the underwriting phase such as, for example, the details of the ratings (i.e., the individual scores of the rating engine), the details of the collateral linked to the credit, the information on the counterparty, the details of the credit. (i.e., amortization plan, interest rates, ...)

If the credit is not digitally originated, it can still be put in the blockchain, but it will be necessary for the originator to certify the truthfulness of the information, introducing a non-negligible operational risk / fraud risk which can however be mitigated by the use of data quality controls that allow better monitoring of the consistency and correctness of the data.

The information included in the blockchain is made unchangeable and a timestamp is associated with them. This generates several advantages, enabling the players in the blockchain to have complete visibility of information with maximum detail, making the information immutable, reducing the risk of data loss / alteration, facilitating due diligence activities as described in the next chapter. Last, any information update is communicated to all participants in the blockchain, thus making the communication process timelier and more transparent.

\subsubsection{Structuring the securitisation}

This phase is one of the most complex as it involves the participation of numerous actors and a long series of activities to be carried out, the most important of which is, without doubts, the Due Diligence.

This activity includes a series of very detailed analyses on the underlying assets, counterparties, and collaterals aimed at confirming the compliance of the credit pool with the specifications of the operation to be carried out.

The analyses are carried out by various actors such as, the arranger, the bank distributor, and the servicer with different degrees of detail based on the information available on the credits.

These are very long and expensive activities but essential to avoid fraud on non-existing assets, already securitised, or qualitatively different and / or incompatible with the characteristics of the securitisation.

Today these activities are carried out manually by sharing information between the actors on traditional channels (i.e., mail, files, etc.) which implies operational errors, slowness, incompleteness, etc.

In this phase the blockchain allows to optimize various aspects of fundamental importance:

- Coordination between the actors to avoid double effort on the same analysis: everyone can know the checks in progress by other actors, thus limiting the risk of double effort,

- Speed of analysis: information is readily available to all participants and, if the blockchain platform is equipped with a business intelligence module, it can be providing a great value in analysis

- Uniqueness of the information: having a single database, the risk of misalignment of the analysed data is avoided, thus improving the comparability of results.

\subsubsection{Contract management}

The definition of the contracts governing the securitisation operation is a tricky activity which is characterized by numerous reworking of documents with the consequent need of versioning.

This activity remains manual even on the blockchain but the comparison between the counterparties, thanks to a single environment where contracts can be shared, allows everybody working on the latest version of the documents, relying on a quick tracking of all changes, increasing their visibility and reaching an agreement between the various actors involved.

The improvement of the dialogue between counterparties leads to better transparency within the blockchain actors and the reduction of costly information asymmetries.

\subsubsection{Issuing and settlement}

The phases of issuing the instrument and settling it on the primary market are phases in which the blockchain does not introduce any significant advantages.

Therefore, these activities remain managed according to current operating practices.

\subsubsection{Lifecycle management}

Once the placement is completed, the servicer begins the management of the securitisation.

In this phase, ordinary activities are managed such as, for example:

- debtor's payment collection

- investor's payment (the so-called payment waterfall)

- accounting management (i.e., bank transfer) and accounting checks

- regulatory reporting

- crystallization of collaterals

- management, where required, of NPL processes (monitoring, credit recovery, legal actions)

- management of credit revolving (i.e., commercial papers securitisations)

All these activities are carried out through dedicated applications of the servicer and it would be expensive and futile to replicate these functions in the blockchain platform.

Instead, it is more appropriate to build an integration so that the data residing on the servicer's legacy are shared in real time on the blockchain with all the other participants in the securitisation.

Once the participants have agreed on what type of data need to be shared, these could be stored on a common database in the blockchain and all the actors could access it when needed. 
Often the cost and time spent to have the information available timely with the desired degree of detail is underestimated: the blockchain allows cost reduction and transparency increase removing the information asymmetries between counterparties.

\subsubsection{Monitoring and reporting}

As reported above, the information uploaded to the blockchain can be shared and used at a lower cost than current securitisation management models.

In particular, using a shared database allows to normalize information and increase data consistency, manage a single standard for the data, use only one communication channel, ensure a unique and certified data source, reduce, if not eliminate, the need to reconcile data, as they are natively consistent, reduce the time lags of information availability, speeding up all lifecycle management activities and last automate all internal /market reporting.

There can be different degrees of sophistication depending on the objective of monitoring: from simple data extractions, to business intelligence interfaces for advanced analysis carried out by all the participants in the blockchain. It is therefore possible to carry out continuous low-cost due diligence that allows to keep the risks of the operation under control.

For monitoring purposes, it is possible to define KPIs or trigger events: when they have broken, the participants receive an alert that allows them to activate remediation actions. For example, in the securitisation of revolving credits, it is possible to verify that the composition and geographical / sectoral / qualitative concentration limits remain, during the entire life of the transaction, the same as those that had been defined at the beginning. If these limits are violated, all participants would be aware of them and promptly activate the planned remediation actions.

Sharing information also benefits product pricing as all investors are able to carry out real-time assessments of the value of the securities. Indeed, pricing models need accurate data to calculate the correct price: in a top-level integration, pricing engine could be integrated directly to the database of the blockchain, making the pricing operation easier, cheaper and faster. The improvement in pricing can generate narrow spread, for the benefit of the market transaction.

Also, the investors can access specific data of the securitisation: this could be a distinctive characteristic compared to the ordinary securitisation. The investors always want to understand where their money are allocated and the transparency guaranteed by the blockchain allow them to prefer this type of securitisation to the others.

\subsection{Blockchain: Benefits \& Limits}

As discussed in the previous section, a key point of blockchain technology applied in securitisation would be the establishment of ecosystem of peers, enabling a "many-to-many" relationship, reducing the barriers to entry for both transferors and final investors (GATTESCHI, LAMBERTI, DEMARTINI, PRANTEDA, SANTAMARIA, 2018). A blockchain-based platform, especially for the invoice securitisation, could represent a cutting-edge tool for Small Medium Enterprise to obtain credit or funding for their invoices. A significant number of SME that are currently looking for funding cannot access to the securitisation market acting as a transferor for their invoices because of the regulatory limitation, and consequently have to rely on another financing instrument (e.g., advance invoice financing) which are significantly less effective.

A blockchain-based platform has the possibility to enable SME to access to securitisation (IBM, 2019) without the need for guarantees or collateral to report to the Central Credit Register of the banking circuit, because it would allow to certify the successful transfer of individual invoices to a bank or other intermediary, allowing immediate control by the transferee and effectively overcome the phenomenon of multiple transfer of the same invoice. The effectiveness of such "certification service" become more significant the more is the number of the participants.

Also, on the other side of the market, private investors could benefit from such a platform because the efficiency in manage the transactions and the digital assets could allow the fragmentation of the financial asset allowing the distribution of securitisation directly to individual private investors instead to Representative of Noteholders only.

Another benefit of a blockchain-based solution is the efficiency that can bring to the ecosystem (ABADI, BRUNNERMEIER, 2018). The process efficiency is basically generated by three aspects.

The first one is establishing trust among the actors: a blockchain-based platform could introduce a new model based on incentives, not only economical but also reputational in order to guarantee the transparency along the chain, potentially leading to disintermediate the intermediaries. Within this ecosystem, it's again easier for smaller or private investor to access to these products, increasing the audience. It's obvious that every member has to accept the platform standard in order to guarantee a controlled access to the network.

Secondly, automation through smart contract: introducing a blockchain-based solution for securitisation, implies the digitalization of all the asset from which it is composed the securitised product. By implementing what we can define a "digital asset", a digital representation of the real financial asset, it is possible to manage and historicized all its life cycle, included all the transfer of property. This allows not only to make natively available all the history of the asset, starting from an Invoice or an NPL to the securitised tranche, which can significantly benefit also the reporting process, but also to build on top of that, any kind of business logics. In a blockchain based application, these business rules are encoded in the so-called Smart Contracts, a self-executing code running on the decentralized blockchain network. These advantages are native in a blockchain-based application and doesn't require for the implementation of other technologies on top.

Last but not least, data certification through Oracles: besides the data stored immutably in the distributed ledger, a blockchainbased application is able to invoke certified external data sources, the so-called Oracles, in order to validate and transmit data from external sources to blockchain systems to be elaborated by the Business logic. In a blockchain based solution for securitisation, a possible Oracle could be "Agenzia delle Entrate" (the Italian tax authority) to certify the validity of the electronic invoice as potential underlying of the Securitised asset. This capability allows to significantly reduce due diligence operations, consequently timespan, and intensify the control frequency.

A key theme to be addressed in the realization of a blockchain based solution is related to the identification of the actors that create the ecosystem. 
The securitisation process, as highlighted in the previous paragraphs, is complex and involves the participation of a large number of actors with specific tasks in different stages of the process. To define the ideal number of roles that have to be implemented for the solution, it's necessary to take into consideration the trade-off of two aspects: the first one is Operative Complexity, which guides to keep a moderate number of actors involved.

Because more actors are involved, higher would be the number of interactions and the functional paths to be managed by such a platform. Secondly, Data Security, which guides to increase exponentially the number of actors involved, because the higher is the number of participants, the higher is the effectiveness of the certification guaranteed by the actors that operate in the network

It is implied that according to the different steps of the securitisation process to be automated, the actors involved have to participate with a role in the platform.

The second key topic to be addressed is the choice to rely on a permissionless or permissioned blockchain for enterprises.

Permissionless blockchains (also called public blockchains) are open to anyone to read, write transactions to, and participate in the consensus process. Permissionless blockchains have several advantages.

First of all, entry costs for new participants are minimal, because the entire infrastructure is already available for all the actors. On the other hand, no central entity controls the proceedings of a public blockchain, the network is spread across all the different nodes and the distribution is concrete.

But it has also the disadvantage that every transaction involves a fee, generally the transaction speed is very low, and moreover it's not possible to customize, based on individual request, the governance structure and the business logic on top.

A permissioned blockchain (also called private blockchain) generally has more applicability for enterprise solution, specifically for financial services, because it includes the ability to keep certain information and transactions private, with different levels of access for different parties, in this way the actors have the possibility to expose some information to some actors and hide other information to other roles. It allows faster transactions and the ability to scale up transactions and data and it gives the possibility to tailor the smart contract on participants' needs.

As a downside, the whole infrastructure has to be set-up with costs proportionate to the ecosystem dimension in order to guarantee the decentralization and immutability of the ledger; at least one node to each actor role has to be set-up.

Third point to be analysed and addressed, in order to implement a solution suitable for participants needs, is platform governance, which implies the management and maintenance of the business logic and the rules that govern the access to the platform.

\section{NPL Securitisation}

\subsection{NPL Context in the European and Italian Market}

As already mentioned, from an initial analysis of the European and Italian scenario, securitisation operations are down compared to previous trends. However, the Italian banking system seems to have realised that through this instrument it is possible to significantly improve pricing levels compared to a traditional assignment of credits simply by virtue of a growing response of NPL stocks. The slow recovery of recent times represents an awareness of the importance of the securitisation instrument. Among the factors that can be considered for facilitating this recovery there are the implementation of a complete and timely regulatory framework on how to carry out the operation, the galloping technological and financial innovation, the need for banks to overcome the risk of asset concentration in an increasingly competitive environment, and the internationalisation of financial markets.

This problem has affected the entire Europe, particularly Italy, where the volume of credit deteriorated NPL is the highest in the continent.

The market for non-performing loans has broken down in Europe after the lockdown period. In fact.in the first half of the year total transactions for 30.4 billion euros were concluded, of which $89.8 \%$ between Italy (18.2 billion euros) and Greece (9.1 billion). The 30.4 billion are compared with the 41.8 billion euro deal of the first half of 2019 and the record of 105.2 billion in the first six months of 2018 (Bebeez, 2020).

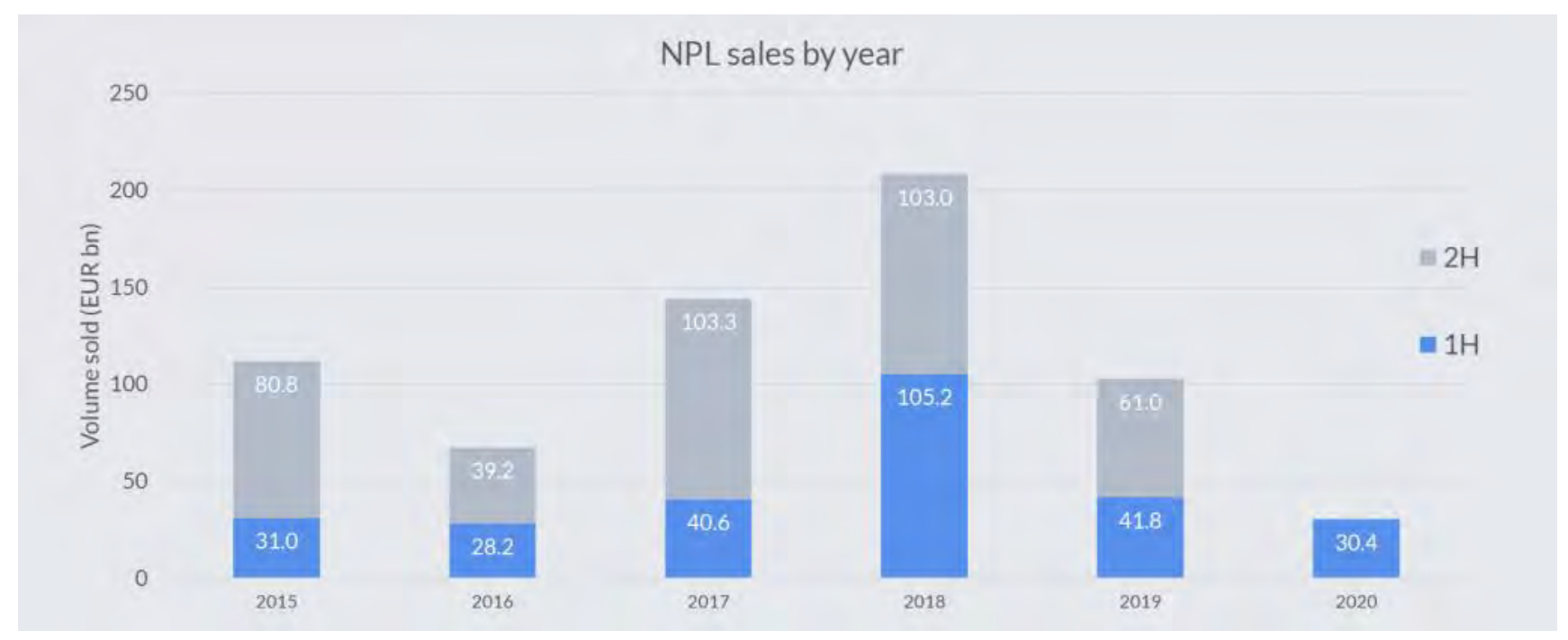

Fig. 6 - European overview - NPLs sales by year. Source: Debtwire NPL Database

Since 2015, Italy has recorded transactions with a gross turnover of over 130 billion Euro which suggests a strong Italian presence in the Non-Performing Loan sector. 


\subsection{As-Is process of NPL Securitisation}

An initial analysis of the phenomenon of NPL securitisations in the Italian and European context highlights the issue of credit quality which has been the subject of discussion in recent years and of numerous regulatory interventions, but still today it is one of the most complex problems to solve.

Among the elements that directly affect the theme of Non-Performing Loans, we have to consider the quality of the securitisation processes and costs of managing volumes of securitised loans.

In this respect, it is necessary to assess the position of banks in order to improve the securitisation process and the credit management capacity.

Changes will be needed in the models and operational processes that securitisation cannot fully guarantee.

Therefore, the main transformation elements of the models and operational processes on which banks will have to intervene are containment of NPL input flows, the increase in recovery performance and the exploitation of the underlying collateral.

Banking institutions are now aware of the importance of achieving, and subsequently maintaining, a high level of quality of data relating to the Non-Performing Loan portfolios to ensure consistency, stability, completeness, accuracy and, in general, reliability of the database on which a plurality of important stakeholders must, now more than ever, rely. In addition to the Management functions of the bank, the main users of the data are: the managers of the recovery process, Credit Risk Management, the Supervisory Bodies, potential buyers and investors, and advisors and rating companies.

In addition, as a financial operation, securitisation can meet multiple goals and produce a wide range of benefits for both stakeholders from a broader and economic point of view.

The originator and the investors derive the greatest benefits for securitisations.

There are several reasons that can make securitisation an attractive form of financing for the originator. In the international experience of securitisation, two main categories of possible benefits for the originator have been identified:

- diversification of collection sources

- rebalancing of the financial structure

Regarding the first point, securitisation gives the possibility to smaller companies, or with low credit standing, to access this form of financing, which is also more efficient when compared to alternative channels.

The reasons for this higher efficiency and lower cost lie in the ability of the issuer, through securitisation, to issue securities that have a higher rating (mainly thanks to the underlying credit enhancement structure) compared to other credit instruments that would only be based on the originator's rating.

The other advantage is, as already mentioned, the rebalancing of the financial structure. In fact, securitisation allows the removal of assets from the company balance sheet and the demobilisation of medium to long-term assets, which may for example, allow the originator to increase liquid assets and rebalance the ratio of current assets to current liabilities.

From the point of view of final investors, the securitisation transaction may offer benefits in terms of adaptability and flexibility to different financial needs, portfolio diversification, risk sharing and tailored investments

\subsection{Data Quality}

In relation to the issue of transformation of the models and operational processes on which banks will have to intervene, it is necessary to address the issue of the quality levels of the information. This evidence also emerges from an analysis of recent securitisation of Non-Performing Loan portfolios, revisions of internal recovery processes, increased use of external servicers, the disclosure requests by the Supervisory Board and the revisions of valuation processes, which are also related to the introduction of IFRS 9. (REPLY AVANTAGE, 2019)

Data Quality, both from a Business and IT point of view, has an important influence on the assessments of the various stakeholders with an overall effect of creating value for the bank.

This is particularly relevant in the estimates of value adjustments for balance sheet purposes, in the determination of the prices applied to the disposal transactions and the associated additional losses from disposal, for the definition of the cost of securitisation transactions, evaluating the impact on Loss Given Default (LGD) as a consequence of the level of effectiveness of recovery processes, estimating the costs of having to take remedial actions such as arrangement of credit sales that require the involvement of external expertise to fill the information gaps represented by lack/incorrect/outdated information.

The factors that, up to now, have impacted the level of the quality of the data and the difficulty and onerousness of the processes of remediation are the result of a negative concatenation of events favoured from complex operating environments.

These include in particular the high number of positions accumulated during years of negative economic cycle (hundreds of thousands at level system); a general inadequacy of the size of the facilities dedicated to the management of non-performing loans compared to the amount of positions generated by the system in the presence of operational inconsistencies, including at the level of servicers, for the management of the power supply of the systems, both at an early stage and in the day-to-day management of updates; the incompleteness of the paper documentation or the information base as a result of numerous aggregation operations, data migration, transfer of locations, territorial reorganisations, etc.; the high number and diversity of information that is often managed by a plurality of management systems; the presence of gaps in the file management and filing systems of the contract documentation, which is not yet fully managed on a digital basis; the processes for updating valuations related to real estate guarantees and their transposition into management systems.

In this sense, the stakeholders involved in the securitisation process are investment-oriented to speed up and improve data quality through, for example, outsourced services, also leveraging the recent guidelines provided by the BCE, which marks a gradual exit from bank perimeters ${ }^{1}$.

${ }^{1}$ The European Central Bank (ECB) has decided that the loan-level data reporting requirements of the Euro system collateral framework will converge towards the disclosure requirements and registration process for securitisation repositories specified in the Securitisation Regulation 
This analysis shows a general orientation of capital market operators towards technological platforms that allow the use of a computer program (i.e., an application) and the access of several entities to the same interconnection network (intangible), as such infrastructures may facilitate the interaction of multiple purchase or sale interests of financial instruments related to NPL or UTP loans.

Only in recent times, in fact, the integration of technological mechanisms appears able to allow the systematic use of new computer architectures (including the c.d. blockchain technology) to facilitate the meeting of supply and demand and to support access to relevant information campaigns (so-called big data), with the effect of supporting the purchase and sale transactions in the reference a mass of markets.

\subsection{Blockchain Benefits for NPL Securitisation}

The analysis carried out revealed several areas in the operations of the Securitisation, on which blockchain technology could intervene:

- Due Diligence and data quality

- Implementation of organisational processes

- Network Information Systems

- Network Actors involved in the platform

The activity of Data Quality and Due Diligence, both from an IT point of view (such as Loan Data Tapes) and from a documentary point of view, has an important influence on the assessments of the various stakeholders with an overall effect of creating value for the bank. Preventive due diligence (cd. pre-acquisition due diligence) plays an important role in securing the securitisation of intermediaries.

The factors that could benefit from the use of the blockchain and that until now, have impacted on the level of data quality and on the difficulty and onerousness of the remediation processes are the result of a negative chain of events favoured by complex operating environments generally linked to the presence of operational inconsistencies, the incompleteness of paper documentation or the information base as a result of numerous aggregation operations, data migration, relocations, territorial reorganisations, etc., the high number and diversity of information, the weakness in management systems documentation and archiving practices, and so on.

Most originators, including many small and medium-sized banks, are not structured to carry out this activity themselves.

Therefore, there is an issue with solving some problems related to internal data management processes.

The European and Italian guidelines ${ }^{2}$ also reviewed the issue of NPLs and it turned out that more accurate management is more profitable than a quick sale.

The servicer/outsourcer is still in play for this precious and strategic activity of enrichment and verification of the information as the instruments are effective for an accurate appraisal independently from the fact that the institute decides to make it an internal management or to assign the task to an outsourcer.

It is necessary to enrich and segment the Loan Data Tape (LDT), the database that must contain data relating to debts and debtors. The most relevant information to update positions and related collateral and personal information continues to be cadastral information, verification of the presence of negative acts (complaints and injurious), the state of enforcement and insolvency procedures, and up-to-date estimates of value.

And here servicers, debt recovery companies and commercial information can become protagonists in this activity and propose themselves in a competitive way to the market if they invest in technology to speed up and optimize the activity of Data Remediation.

As the operating systems of banks are based on accounting and not management, independent servicers need to take advantage of this opportunity.

In addition, the operators of the recovery sector can "give the shock" in this strategic area to the banking world-financial: they must propose to institutions to create specialized units composed not only of legal figures but also of recovery professionals as are the Italian companies with License.

The key in the Implementation of organisational processes is TIME.

Regarding the securitisation organisational process, it is important to focus on the implementation of the process of processing practices that is a complex information investigation process that must be constantly developed to avoid anomalies and ensure necessary customisations for the customer.

For information and recovery companies, a success factor is their ability to differentiate these information processes from competitors, always looking for new investigation and evaluation strategies.

Information companies wishing to offer services and be competitive have two critical aspects to focus on. The first one is to fully understand the needs of customers and have the ability to organize and provide timely answers. The second one is to be able to adapt operational processes to new survey needs by optimizing time and resources.

To address and resolve these two challenges, companies must focus not only on skills but above all on software and solutions to support operational activity in view of Network Information Systems and Network Actors involved in the platform. It is the management of information processes that can make a difference.

(Regulation (EU) No 2017/2402). The ECB has taken this decision with a view to promoting efficiency and standardisation in the securitisation market. (Regulation (EU) No 2017/2402)

2 The European Central Bank (ECB) has decided that the loan-level data reporting requirements of the Euro system collateral framework will converge towards the disclosure requirements and registration process for securitisation repositories specified in the Securitisation Regulation (Regulation (EU) No 2017/2402). The ECB has taken this decision with a view to promoting efficiency and standardisation in the securitisation market. (Regulation (EU) No 2017/2402) 
A process is a set of activities carried out automatically or with manual intervention. The process environment of the blockchain technology allows you to configure the information processes and related steps that can be executed automatically or require operator intervention.

The operation of defining a process of "informative investigation" is carried out and created in a simple way by a user even nontechnical that "models" the input files provided by the customer through a system of Dictionary and "configures" the path of investigation to do. Important in this sense is the role of the actors involved in the process of securitisation that could intervene, through the blockchain to regulate the internal processes of management of the life cycle of the securitisation. The blockchain aims to connect the entire ecosystem involved in securitisation operations and to allow the various actors to interact in the different phases in a transparent way ensuring the following benefits of blockchain: more data quality, process safety, traceability of flows, reduced processing times and paper use. The DLT offers real benefits for the stakeholders of the transactions, providing them with a better level of interaction, greater process sharing and trust, as well as a higher degree of financial asset collateral.

Therefore, we can summarize here the benefits that the securitisation process with NPL and UTP underlying would have with the application of the blockchain:

- Increased productivity and efficiency

- Continuous monitoring and better control

- Flexibility and simplification in process design

- Digitalisation and transformation of data into digital format

- The decentralization, for which the images are distributed between more nodes in order to guarantee the computer security of the systems

- The traceability of transfers, whereby each step is traceable in its entirety and its provenance is recorded

- Disintermediation, whereby transactions are managed without intermediaries, or without the intervention of trusted central institutions, such as banks

- Verifiability, for which every element of the register is transparent and visible to all, therefore it is totally searchable and has the possibility to be verified

- Immutability of the register, so that the data entered in the register cannot be modified without the consent of the network, for this reason it is often spoken about net neutrality

- Programmability of transfers, so it is possible to program actions that are activated only when certain conditions are already set.

\section{Electronic invoices securitisation}

\subsection{Securitisation Electronic Invoices in Italian Market}

In recent years, there has been an increasing trend in the securitisation of loans with underlying electronic invoices due to the general economic performance and suggestions dictated by the Legislator.

The changes, introduced by the "Destination Italy" decree ${ }^{3}$ and the "Competitiveness" decree to the law on securitisation of receivables, make the securitisation easier and more efficient than commercial receivables increasing the benefits of a trade receivables disposal transaction under the factoring law. However, today operators have two legal instruments at their disposition in order to obtain liquidity using the credits deriving from their own business. The market of the cession of the commercial invoices lends itself to operations of securitisation and, with the emission of asset backed securities (ABS), they will have similar underlying credits that can also theoretically be undersigned for small sizes.

In the Italian market, the phenomenon of securitisation transactions of trade receivables is increasingly widespread with the acquisition on average of a portfolio of 5 million trade receivables through a securitisation vehicle.

In detail, the securitised trade receivables from Italian small and medium-sized enterprises were traded on suitable platforms and originated entirely from them. This new securitisation model requires SMEs to use the platforms offered to sell part of their trade receivables from customers while the investor can then enter into a credit insurance contract with a specialized company. Loan securitisation, which is based on commercial invoices characterized by low risk of default and high turnover, is a process that aims to provide liquidity by transforming illiquid assets into securities, effectively monetizing the asset and creating a new source of financing.

The securitisation of trade receivables sold to third parties through bonds is confirmed as significant, around 8.5 billion euro. And despite limited volumes, innovative solutions are expanding: $+15 \%$ for the use of the credit card as a tool to optimize the working capital which is worth 3 billion euros, $+225 \%$ for invoice trading (the transfer of invoices through digital) amounting to 0.13 billion, while Confirming's first initiatives, the transfer of a supplier payment mandate to a factor that becomes the manager of trade debts, reached 0.5 and 0.01 billion respectively. The possibility of securitisation of a commercial invoice obviously assumes that the more value the greater the payment times that customers allow themselves.

\subsection{Limits of the process}

In the context of the securitisation process having invoices as underlying, different limits are identified:

1. Non-standard checking of invoices

2. Timetable Transactions for resolving securitisations

${ }^{3}$ D.L. December 23, 2013, n. 145, converted with modifications from the Law 21 February 2014 (ITALIAN GOVERNMENT, D.L. 145/2013 "Destination Italy", 2013) which introduced, inter alia, the possibility for the securitisation company to subscribe to bonds or other financial instruments not representing share capital, simplified the formalities required for the enforceability of the assignment of the receivables subject to securitisation, (ii) the D.L. 24 June 2014, n. 91 (ITALIAN GOVERNMENT, D.L. 91/2014 "Competitiveness Decree", 2014) which introduced in our legal system, with some temperaments, the faculty for the securitisation company to grant loans directly to companies (c.d. direct lending), and (iii) last, the D.L. 24 April 2017, n. 50, converted with amendments by Law 21 June 2017, n. 96 , on the securitisation of non-performing loans and the participation of the securitisation company in the risk capital or debt of companies in crisis 


\section{Disposals of multiple credits.}

The first element that emerges from the analysis of the invoice securitisation process, and from its related limits, is a slowdown in the issuing activity (up to 5 days after the transmission) with negative consequences for the sales of invoices processed at the end of the period where the relative receipt is not yet available. The wait from when a company sends the invoice to the feedback obtained can have an impact in the individual phases of the sale. Furthermore, the banking system requires firms to work with the entire turnover and with invoices of certain minimum sizes and the resolution status of securitisation transactions has a long duration. The analysis of these points highlights that necessary solutions should be found to maintain compliance, as at present, with the needs of both the originators and the transferees who may carry out financing activities, management and warranty only if the regulatory and operational prerequisites have been fulfilled.

In particular, we refer to:

- the notification information of the credit assignment

- lack of securing the securitised credits (the indications of invoice payments are already present in the xml format, while the indication of the assignment is not currently envisaged in some cases of assignment of credits which will be implemented in the European standard which however will not be unique)

- the timing of issuing the invoices

- the necessary and mandatory control by the originator to verify the validity of the invoices issued by the transferors at the time of the transfer

- management of securitised credit processes

- lack of a standardization process for securitised invoices

- internal deliberation processes that involve several functions, thus slowing down the time of the deliberation itself

According to the rules of electronic invoicing, it is necessary to provide not only invoices in electronic format, but also to implement the related management process. The latest European directives and the interests of the market highlight the need for a standardized and more controlled process of these types of transactions ${ }^{4}$. However, this process would be onerous and complex for customers who will certainly delay the transfer times with consequences in the receipt of both the loans and the guarantees on the successful outcome of the payment (where provided).

Regarding the limit of the current system of securitisations, the use of this tool and asset sales do not allow the transferee to have immediate control of the nature and life cycle of securitised loans and does not hinder the phenomenon of multiple assignments of the same. Looking ahead in the securitisation market, the possibility of exploiting the full potential of electronic invoices was favourably assessed by implementing a non-competitive platform aimed at reducing the risk of multiple assignments, possibly also using innovative technologies (i.e., DLT). A similar "computer register" would allow to certify, by means of non-manipulable computer processes, the successful transfer of individual invoices to a bank / intermediary, allowing immediate control by the transferee and effectively hindering the phenomenon of multiple transfer of the same. The effectiveness of such a service is greater the greater the extent of the participants: therefore, a non-competitive system initiative would be ideal, which would allow interfacing any front-end platforms of the individual transferees. The combination of the use of electronic invoices, digitally signed and whose issuance and fiscal validity is certified by the blockchain, and a non-competitive computer register of credit transfers at system level can effectively contribute to containing and minimizing the risks of fraud for false invoicing and / or multiple assignments.

\subsection{Blockchain Benefits for Electronic Invoices Securitisation}

The securitisation process would be benefited by informed and partly automated decisions, making it possible to mitigate various forms of risk (double financing and fraud, operational risk and credit risk). This process could be benefited from standardized processes and new technologies that would speed up the times of cession with a cost reduction of emission, sending and archiving of the invoices. Therefore, it should be possible for the originator or the assignee to benefit from a technology enabling the management of securitised credits to be implemented throughout its life cycle. The following table shows the main risks arising from the traditional securitisation operation and the benefits expected from the application of the blockchain.

\begin{tabular}{|l|l|c|c|c|}
\hline \multicolumn{1}{c|}{ RISKS } & \multicolumn{4}{c|}{ BENEFITS } \\
\cline { 2 - 5 } & Flexibility & $\begin{array}{c}\text { Transparency and } \\
\text { Traceability System }\end{array}$ & Increase Network & Standardization \\
\hline Double financing & $\checkmark$ & & & \\
\hline Operational risk & & & $\checkmark$ & $\checkmark$ \\
\hline Credit risk & & & & \\
\hline Fraud & & & & \\
\hline Seller analysis & & & & \\
\hline Performance conditionality & $\checkmark$ & & & \\
\hline
\end{tabular}

Fig. 7 - Traditional securitisation risks offset by blockchain adoption. Source: Reply internal elaboration

\footnotetext{
${ }^{4}$ Directive 2014/55/EU (EU, Directive 2014/55, 2014)with Legislative Decree 148 of 27 December 2018 (ITALIAN GOVERNMENT, DL 148/2018, 2018), which entered into force on 1 February 2019 (articles 6-11) Subsequently, with Measure No. 99370 of 18 April 2019 of the Revenue Agency, the technical rules were defined, so-called Core Invoice Usage Specification (CIUS)
} 
Through the blockchain technology, therefore, a digital platform developed internally or in partnership with a provider of technological services would be offered. The digital mode, together with advanced analytics algorithms, greatly enhances the economies of scale in the onboarding, resolution and servicing of the position. The scheme can envisage the possible involvement of other subjects, depending on the management strategy of the commercial credit purchased, for example credit insurers and / or institutional investors through the securitisation of the credits acquired through the platform (often securitisation is the main strategy, especially when the solution is offered to small businesses).

In this case, the platform enables a relationship between the different actors (several transferors - one transferee), representing a transformation of the traditional securitisation.

The digital platform can free itself from financial intermediaries and offer its own credit advance solution through a system for uploading and buying and selling invoices to be sold, also through the auction mechanism.

This scheme, which creates an originator - transferee relationship (many assignors - many assignees), profoundly innovates the relationship between the actors involved in the assignment of credit. Originators can sell their credits at the best conditions offered by a plurality of potential investors. Investors (who are not necessarily financial intermediaries) can access a large number of invoices representing trade credit portfolios, leveraging the risk assessment carried out by the platform according to sometimes shared criteria and in some cases supported by the guarantee of a company credit insurance. Debtors typically receive notification of the assignment but are frequently not the subject of activities aimed at collecting the credit.

At the end of what is analysed here, the role assumed by blockchain technology in the management of the commercial credit assigned is to strengthen the concept of ownership of the credit and, as such, the beneficiary of subsequent payments by the debtor. On this point, it is right to add that, for some types of contracts, it is envisaged by law that the expiration date and therefore, of collectability of the credit, will be calculated starting from the date of receipt of the invoice by the debtor.

\section{Conclusion}

The benefits provided by the application of the new technology are not limited to the purely, yet fundamental, operational component in managing securitisation and digitalised assets. We have seen how, the strong reduction in what we can generally call "operational risks and costs" of the underlying assets to be managed is sided by an equally relevant reduction in other risks, such as liquidity risk and credit risk.

All this can be directly or indirectly translated into a competitive advantage for the securitisation financial return, that of course can be exploited throughout the entire value chain, specifically for the early birds, that are going to get the wider risk premium spread, as we will see later on.

Looking for example to the main sources of illiquidity previously listed, the blockchain-built securitisation is easily getting rid or significantly reducing transaction costs and discrepancies in evaluation. It can also support a more effective market evaluation and improve the symmetry of information, where a deeper level of information can bring a wiser analysis of the credit situation and score.

We can then propose to group the several components affecting the risk (and consequently the return) of the digitalised asset into two main categories:

A. The "Endemic" component, here including those risk factors that are unaffected or only partially reduced by the introduction of the blockchain technology (i.e., divergence in expectations).

B. The "External" component, representing the risks that can be reduced or offset by the distinguishing traits brought in by the new framework.

Here is a qualitative representation of the effects that the two risk categories can have on the return of an asset assuming a traditional technology adoption curve.

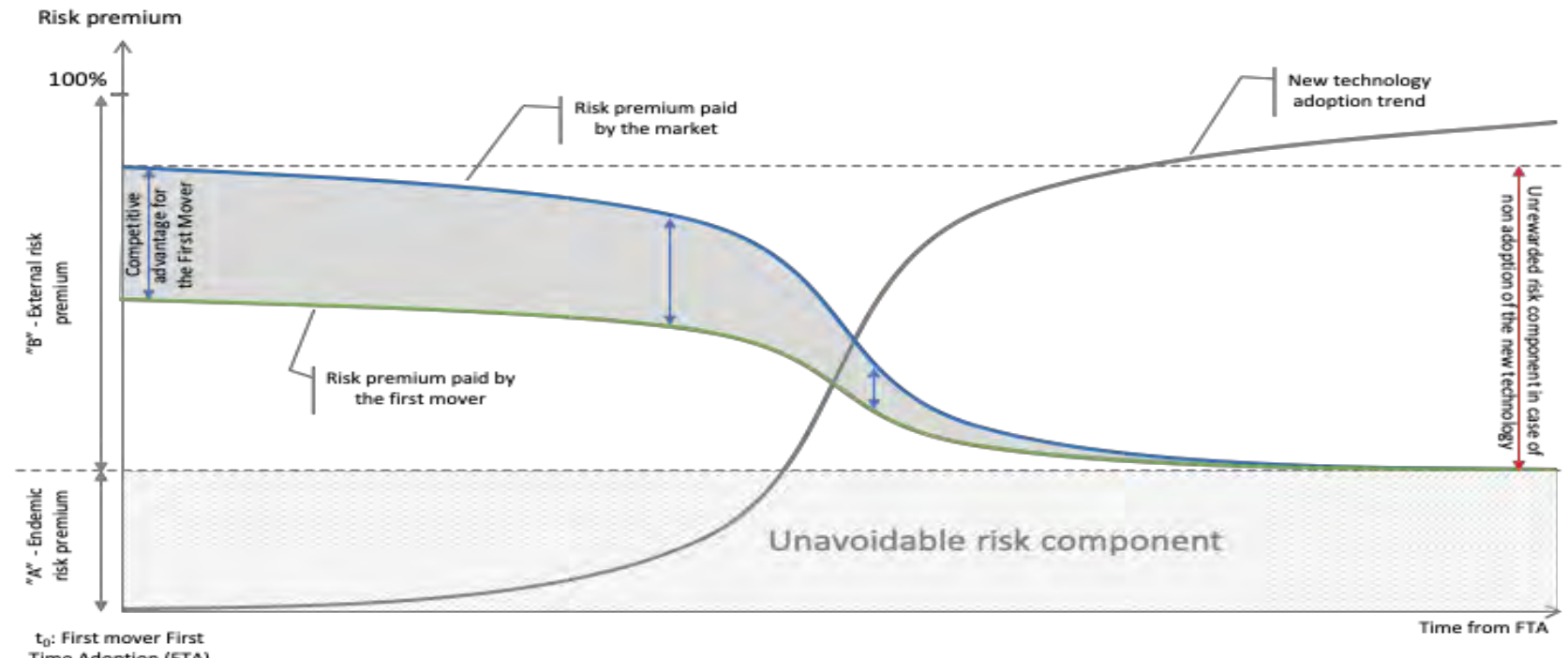

Time Adoption (FTA)

Fig. 8 - Risk vs. model adoption curve. Source: Reply internal elaboration 
The main outcomes of the analysis lead to the following conclusions:

- We assume that the new technology has a standard adoption trend (grey line), slower at the beginning and asymptotically reaching a nearly full coverage. (Of course, there will always be exceptions due to late adopters, peculiar product situations, etc.).

- The risk premium (blue line) paid by the market is going to decrease with the blockchain wider adoption, due to the reduced risk associated to the product, i.e., the reduction of the External risk component (B). The total risk curve is going to finally reach its lowest when meeting the limit of the Endemic component, i.e., the unavoidable risk.

- The early adopters will benefit from a competitive advantage (the grey area) representing the difference between the risk generally associated by the market to the specific product (again, the blue line) and the risk effectively borne by the issuer/holder of the digitalised asset. This component is going to be progressively reduced with the wider adoption of the new technology. The costs associated with the hurdle related to the early adoption process are more than offset by the difference in return.

- For those players who will not join the new technology, the bottom line is an unrewarded risk component (red arrow) given that they are bearing a risk not considered by the market anymore.

The bottom line is that the adoption of the new technology can deeply affect the overall business approach and the risk management of the illiquid assets, reducing their external risk component and finally the related return.

An innovative technology is at the same time a leading contestant in the change to financial markets through the creation of new products, new economic sectors and increasing market accessibility. Digitization and blockchain platforms could transform the whole securitisation market boosting efficiency by establishing trust among actors as well as automation through smart contract and data certification. Securitisation operated as an effective deleveraging instrument in the banking sector, confirming the importance of these financial instruments as an effective funding channel to the economy; however, despite the amount of illiquid assets, European current emissions remain stagnant.

New securitisation regulation 2017/2401 - 2017/2042, which came into effect on January 1, 2019, attempted to strengthen investor's confidence by supporting Simple, Transparent and Standardized securitisations (STS), in contrast to previous opaquer and more complex securitisation transactions. In addition to recent regulatory action, we are witnessing the rise of fintech start-ups and technological firms which are establishing solutions to ensure player awareness on blockchain-based securitisation benefits. As we reported, the benefits provided by the application of new technologies are not limited to the purely, yet fundamental, operational component in managing securitisation and more in general digitalized assets. The strong reduction in what we can generally call "operational risks and costs" of the underlying assets to be managed is joined by an equally relevant reduction in other risks, like liquidity risk and credit risk to mention the more important ones. This is particularly true for first movers in new technologies adoption because they could have a significant competitive advantage in connection to the risk premium paid for their issuances. When specifically looking at the credit sector, the distinctive features of this type of deal compared to the traditional offer are listed in the chart below.

\begin{tabular}{|c|c|}
\hline FEATURES & DESCRIPTION \\
\hline \multirow[t]{2}{*}{ FLEXIBILITY } & $\begin{array}{l}\text { Credit flexibility for any type of SME without the need for guarantees or collateral } \\
\text { thout reporting to the Central Credit Register of the banking circuit; ease of use of the } \\
\text { vice. }\end{array}$ \\
\hline & $\begin{array}{l}\text { Speed of placement of a credit (in fact, once the offer is accepted by the platform, the } \\
\text { rchase is generally settled within } 48 \text { hours). }\end{array}$ \\
\hline \multirow{4}{*}{$\begin{array}{c}\text { TRACEABILITY SYSTEM AND } \\
\text { TRASPARENCY }\end{array}$} & $\begin{array}{l}\text { Cost transparency in a market with multiple investors competing on the value of the } \\
\text { restment. }\end{array}$ \\
\hline & $\begin{array}{l}\text { Increase in the efficiency of administrative processes, thanks to the automation of the } \\
\text { ases of generation, sending, receiving the credit. }\end{array}$ \\
\hline & $\begin{array}{l}\text { Increased security, thanks to electronic signature processes that allow the sender and } \\
\text { ipient to guarantee the integrity and authenticity of the credit. Automation of validation } \\
d \text { verification processes by integrating the document data with the company's } \\
\text { inagement systems. }\end{array}$ \\
\hline & Immediate control of credits to hinder the phenomenon of multiple transfers. \\
\hline \multirow{5}{*}{ SAVING COST AND TIME } & $\begin{array}{l}\text { Significant reduction in the costs of issuing, sending and archiving credits with } \\
\text { portant savings compared to traditional issuing processes. }\end{array}$ \\
\hline & $\begin{array}{l}\text { Reduction of collection times thanks to the increase in the efficiency of the credit issue } \\
\text { d receipt processes and the elimination of credit generation and accounting errors. }\end{array}$ \\
\hline & $\begin{array}{l}\text { Sale of thousands of small credits, thanks to the power of technology. In this way it is } \\
\text { ssible to manage seasonal peaks of activity. }\end{array}$ \\
\hline & Optimization of securitisation resolution operations. Resolve in a couple of days. \\
\hline & $\begin{array}{l}\text { Time compression in the credit issue and transmission phase thanks to digitalization / } \\
\text { ckchain. }\end{array}$ \\
\hline INCREASE NETWORK & Management also of single foreign customers. \\
\hline STANDARDIZATION & $\begin{array}{l}\text { Standardization of the securitisation process, which aims to provide liquidity by } \\
\text { nsforming illiquid assets into securities, effectively monetising the asset and creating a } \\
\text { w source of financing. }\end{array}$ \\
\hline
\end{tabular}

\section{Fig. 9 - Blockchain main advantages in the credit issuing process. Source: Reply internal elaboration}

The paper has explained how the introduction of blockchain could reshape securitisation process and participants with concrete benefits along all the value chain, especially for credit origination, structuring phase, lifecycle management, monitoring and reporting with reduction in terms of costs and time, increase in transparency and data quality. It was pointed out that blockchain, in 
preparation for an increase in non-performing loans (NPLs) at this historical moment of the economy, could cope with this new wave of management of NPLs so as to bring the technological benefits of blockchain to the world of investors and financial services. Finally, with the increase in securitisation of invoices and with a greater awareness of the legislator, the use of blockchain technology has developed through platforms that then offer initiatives specifically targeted to the segment of small enterprises, to which more flexible services are provided, such as: a shorter period of time between the preparation of invoices and the provision of liquidity; the choice of invoices to be credited; the confidentiality of debtors.

All this leads the way to a deeper analysis of the blockchain-based securitisation process, with the target to apply the new technology to a wider perimeter of products. The objective is aiming to design and to bring to economic and financial community an "ecosystemic" tool through innovative approaches that can clearly and easily present the widespread returns available for all involved players.

Paolo Fabris, Valerio Begozzi, Angelo Santarossa, Francesco Dammacco, Gianmarco Fagiani, Riccardo Rostagno, Chiara Frigerio

\section{References}

- Abadi, Joseph, and Markus Brunnermeier. Blockchain economics. No. w25407. National Bureau of Economic Research, 2018.

- Afme (2020). AFME Securitisation data report Q2 2020.

- Akerlof, George A. "The market for "lemons": Quality uncertainty and the market mechanism." Uncertainty in economics. Academic Press, 1978. 235-251.

- Bebeez (2020). Npl Report on 7 Months 2020 del 3 agosto 2020.

- Caselli, Stefano, and Giampaolo Gabbi. Il credito e la crescita: Banche e finanza per le imprese. EGEA spa, 2020.

- CeTIF (2020). Elaboration from International Monetary Fund, (2020). Private debt, loans and debt securities. Bank of International Settlements (BIS) reported in SIFTMA, (2019). Capital Market Fact Book. Deloitte, (2019). Art \& Finance.

- Cominelli, Valentino. "PropTech 3.0. blockchain and smart contracts disruption for the real estate industry: development of an innovative business model for tokenization in the NPL market." (2020).

- Confalone, Paolo Roberto. "Analisi sulla supply chain aziendale e sull'utilizzo della tecnologia blockchain." (2020).

- Davidson, Sinclair, Primavera De Filippi, and Jason Potts. "Economics of blockchain." Available at SSRN 2744751 (2016).

- Deloitte (2017). Applying blockchain in securitisation: opportunities for reinvention

- Esma (2020). List of Securitisations notified to ESMA as meeting the requirements of Articles 19 to 22 or Articles 23 to 26 of Regulation (EU) 2017/2402.

- EU (2014). Directive 2014/55.

- EU (2017). European parliament and council, EU Regulation 2017/2401 - 2017/2402.

- European Parliamentary Research Service (2020). Blockchain for supply chains and international trade

- Gatteschi, Valentina, et al. "To blockchain or not to blockchain: That is the question." IT Professional 20.2 (2018): 62-74.

- Gervasoni Goware \& Guerini Next, (2020). Come la tecnologia cambierà il rapporto banca-imprese

- Hofmann, Erik, Urs Magnus Strewe, and Nicola Bosia. Supply chain finance and blockchain technology: the case of reverse securitisation. Springer, 2017.

- Hyperledger Fabric (2020). Smart Contract and Chain Code

- Ibm (2019). Blockchain can help increase access to credit for SMEs

- Italian Government, I. (2013). D.L. 145/2013 "Destination Italy".

- Italian Government, I. (2014). D.L. 91/2014 "Competitiveness Decree".

- Italian Government, I. (2018). DL 148/2018.

- Moody's (2019). Blockchain improves operational efficiency for securitisations, amid new risks

- Poonam Garg, Technological Forecasting and Social Change, 120407 (2020). Measuring the perceived benefits of implementing blockchain technology in the banking sector

- Reply Avantage. (2019). Analysis on European Commission institutional paper "European Economic Forecast - Autumn 2019". Displayed data and Forecasts for the EU refer to the EU28, including UK.

- Tafolong, Tedongap (2017). Illiquidity and Investment Decisions: A Survey. Working Paper: Cross Asset Investment Strategy.

- Tapscott, Alex, and Don Tapscott. "How blockchain is changing finance." Harvard Business Review 1.9 (2017): 2-5.

- Treleaven, Philip, Richard Gendal Brown, and Danny Yang. "Blockchain technology in finance." Computer 50.9 (2017): 14-17.

- Wüst, Karl, and Arthur Gervais. "Do you need a blockchain?." 2018 Crypto Valley Conference on Blockchain Technology (CVCBT). IEEE, 2018.

- Xinying, US Patent App. 16/888,421 (2020). Blockchain-based financing

- Zheng, Zibin, et al. "Blockchain challenges and opportunities: A survey." International Journal of Web and Grid Services 14.4 (2018): 352375 . 


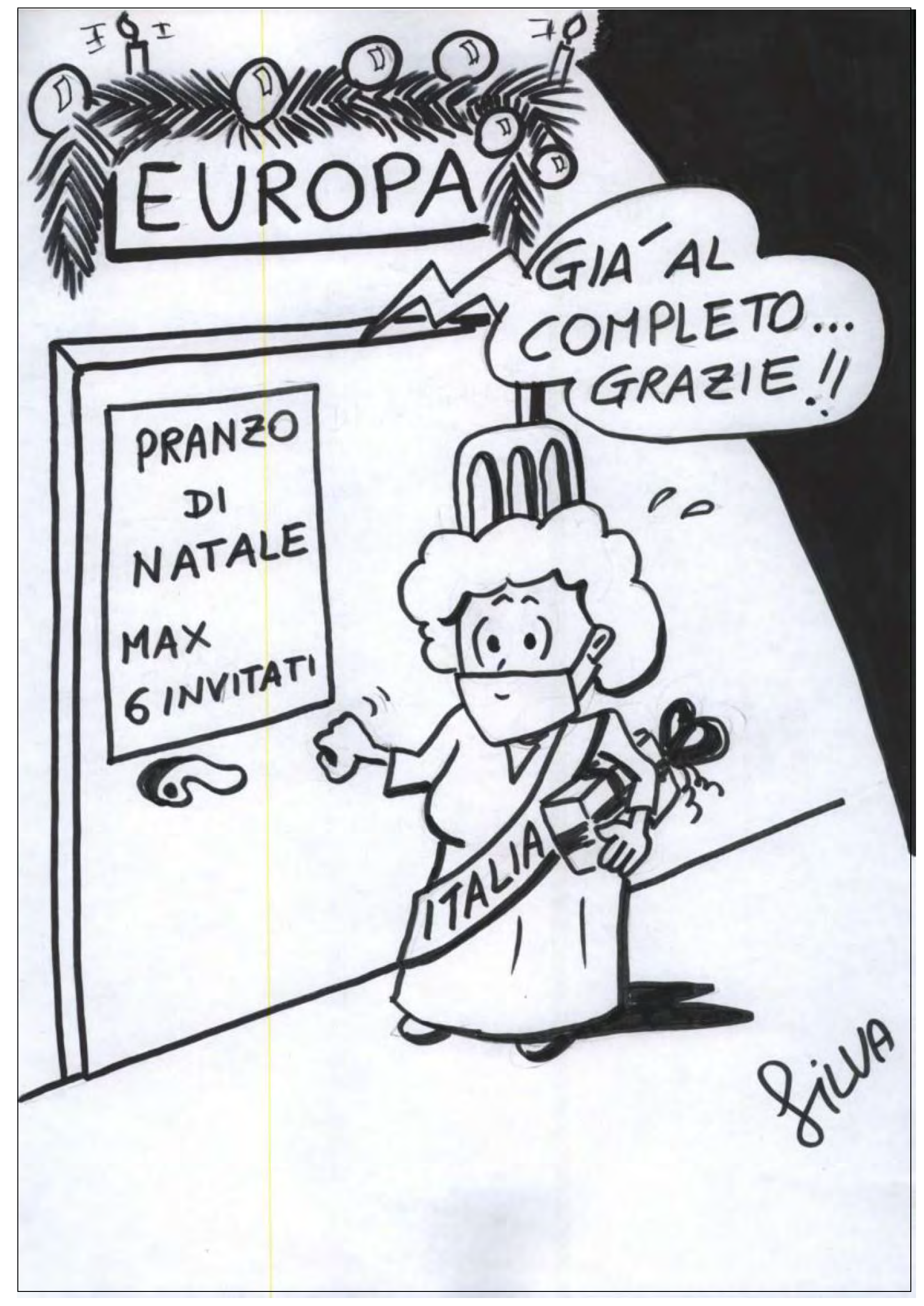


Risk Management Magazine

Anno $15 n^{\circ} 3$ Settembre - Dicembre 2020

Direttore Responsabile (Chief Managing Editor)

Maurizio Vallino

Condirettore (Deputy Managing Editor)

Corrado Meglio

Editorial Board

Giampaolo Gabbi - Chief Editor Business Economics Area (SDA Bocconi); Paolo Giudici

- Chief Editor Statistical Economics Area (Università di Pavia); Daniel Ahelegbey (Università di Pavia); Raffaella Calabrese (University of Edimburgh); Robert Eccles (Oxford University); Franco Fiordelisi (University of Essex); Pier Giuseppe Giribone (Università di Genova); Gulia Iori (London City University); Richard M. Levich (New York University); Michèle F. Sutter Rüdisser (University of San Gallen); Peter Schwendner (ZHAW Zurich University of Applied Sciences); Alessandra Tanda (Università di Pavia).

\section{Scientific Committee}

Arianna Agosto (Università di Pavia); Ruggero Bertelli (Università di Siena); Paola Bongini (Università Milano Bicocca); Anna Bottasso (Università di Genova); Marina Brogi (Università La Sapienza di Roma); Ottavio Caligaris (Università di Genova); Rosita Cocozza (Università di Napoli); Costanza Consolandi (Università di Siena); Simona Cosma (Università del Salento); Paola Ferretti (Università di Pisa); Andrea Giacomelli (Università di Venezia); Adele Grassi (Vice Presidente APB); Valentina Lagasio (Università La Sapienza di Roma); Duccio Martelli (Università di Perugia); Laura Nieri (Università di Genova); Pasqualina Porretta (Università La Sapienza di Roma); Anna Grazia Quaranta (Università di Macerata); Enzo Scannella (Università di Palermo); Cristiana Schena (Università dell'Insubria); Giuseppe Torluccio (Università di Bologna).

Vignettista: Silvano Gaggero

Proprietà, Redazione e Segreteria:

Associazione Italiana Financial Industry Risk Managers (AIFIRM), Via Sile 18, 20139

Milano

Registrazione del Tribunale di Milano $n^{\circ} 629$ del 10/9/2004

ISSN Print 2612-3665 - ISSN Online 2724-2153

DOI $10.47473 / 2016 \mathrm{rrm}$

E-mail: risk.management.magazine@aifirm.it; Tel. +39 3896946315

Stampa

Algraphy S.n.c. - Passo Ponte Carrega 62-62r 16141 Genova

Le opinioni espresse negli articoli impegnano unicamente la responsabilità dei rispettivi autori

SPEDIZIONE IN ABBONAMENTO POSTALE AI SOCI AIFIRM RESIDENTI IN ITALIA, IN REGOLA CON L'ISCRIZIONE 


\section{prometeia}

\section{*1 PER LE SOLUZIONI DI BALANCE SHEET RISK MANAGEMENT}

Prometeia ha vinto il premio per le migliori soluzioni di Balance Sheet Risk Management nell'edizione 2021 di RiskTech100 ${ }^{\circledR}$ di Chartis Research, il più prestigioso e completo sondaggio tra i tech vendor internazionali in ambito Risk Management e Regulatory Compliance. Quest'anno Prometeia è entrata anche nella top 30 della classifica globale, diventando cosi i quinto player RiskTech dell'Unione Europea. Questo importante riconoscimento arriva dopo il recente XCelent Award per Advanced Technology \& Analytics for Balance Sheet Management and ALM. Successi che confermano come il nostro contributo di innovazione sia sempre più riconosciuto e apprezzato, anche e soprattutto in circostanze eccezionali come quelle che stiamo vivendo.

www.prometeia.com 